\title{
Majority rule in the absence of a majority
}

\author{
Klaus Nehring*and Marcus Pivato ${ }^{\dagger}$
}

September 5, 2011

\begin{abstract}
What is the meaning of 'majoritarianism' in a judgement aggregation problem when the propositionwise majority view is logically inconsistent? We argue that the majoritarian ideal is best embodied by the principle of supermajoritarian efficiency (SME): it is acceptable to overrule a supermajority in one proposition, but only if this allows us to agree with a larger supermajority in some other proposition. We show that for certain judgement spaces, the SME outcome is generically unique. For most spaces, however, there are a range of SME judgement aggregation rules. We show that SME, along with separability, characterizes the class of additive support rules. We then show that the median rule is the unique additive support rule which satisfies reinforcement, upper hemicontinuity, and a weak regularity condition; this can be seen as a judgement aggregation analog of a classic result by Young and Levenglick.
\end{abstract}

\section{Introduction}

Let $\mathcal{K}$ be a finite set, representing a collection of propositions. A judgement on $\mathcal{K}$ is an element $\mathbf{x} \in\{ \pm 1\}^{\mathcal{K}}$, where $x_{k}=1$ if $\mathbf{x}$ 'asserts' proposition $k$, and $x_{k}=-1$ if $\mathbf{x}$ 'denies' proposition $k$. A judgement space is a subset $\mathcal{X} \subseteq\{ \pm 1\}^{\mathcal{K}}$; typically $\mathcal{X}$ the set of judgements which are 'admissible' or 'logically consistent' according to our interpretation of the elements of $\mathcal{K}$.

For example, let $A \in \mathbb{N}$, and let $\mathcal{A}:=[1 \ldots A]$ represent a set of $A$ social alternatives. Let $\mathcal{K}:=\left\{(a, b) \in \mathcal{A}^{2} ; a<b\right\}$. Then any judgement $\mathbf{x} \in\{ \pm 1\}^{\mathcal{K}}$ can be interpreted as a complete, antisymmetric binary relation (i.e. a tournament) $\prec$ on $\mathcal{A}$, where $a \prec b$ if and only if either $x_{a, b}=1$ or $x_{b, a}=-1$. (Recall that exactly one of $(a, b)$ or $(b, a)$ is in $\mathcal{K}$.) Now let $\mathcal{X}_{\mathcal{A}}^{\mathrm{pr}} \subset\{ \pm 1\}^{\mathcal{K}}$ be the set of judgements representing transitive tournaments (i.e. strict preference orders) on $\mathcal{A}$; this space is sometimes called the permutahedron. Judgement aggregation over $\mathcal{X}_{\mathcal{A}}^{\mathrm{pr}}$ therefore corresponds to classical Arrovian preference aggregation.

\footnotetext{
*Department of Economics, UC Davis, California, USA. Email: kdnehring@ucdavis.edu.

$\dagger$ Department of Mathematics, Trent University, Peterborough, Ontario, Canada. Email: marcuspivato@trentu.ca. This research was partly supported by NSERC grant \#262620-2008.
} 
Judgement spaces can be used to encode a variety of decision problems. These include: allocating a finite budget over two or more expenditure categories; selecting a committee from a set of candidates; classifying a set of objects into equivalence classes or into a taxonomic hierarchy; designing a communications network; or deciding the truth values of a set of logically interconnected propositions. Nehring and Puppe (2007), Nehring et al. (2011), and Nehring and Pivato (2011a) provide many other interesting examples.

Let $\mathcal{X} \subset\{ \pm 1\}^{\mathcal{K}}$ be a judgement space, and let $\Delta(\mathcal{X})$ be the set of all functions $\mu$ : $\mathcal{X} \longrightarrow \mathbb{R}_{+}$such that $\sum_{\mathbf{x} \in \mathcal{X}} \mu(\mathbf{x})=1$. An element $\mu \in \Delta(\mathcal{X})$ is called a profile, and describes a population of (weighted) voters; for each $\mathbf{x} \in \mathcal{X}$, the value of $\mu(\mathbf{x})$ is the fraction of (weighted) voters who endorse judgement $\mathbf{x}$. The data $(\mathcal{K}, \mathcal{X}, \mu)$ is called a judgement aggregation problem.

For example, let $\mathcal{N}$ be a finite set of voters, and let $\omega: \mathcal{N} \longrightarrow \mathbb{R}_{+}$be a 'weight function' such that $\sum_{n \in \mathcal{N}} \omega(n)=W$ for some $W<\infty$ (reflecting, e.g. the differing expertise or priority of different voters). For all $n \in \mathcal{N}$, let $\mathbf{y}^{n} \in \mathcal{X}$ describe the opinion of voter $n$. The profile determined by this data is the function $\mu: \mathcal{X} \longrightarrow[0,1]$ defined by

$$
\mu(\mathbf{x})=\frac{1}{W} \sum\left\{\omega(n) ; n \in \mathcal{N} \text { and } \mathbf{y}^{n}=\mathbf{x}\right\}, \quad \text { for all } \mathbf{x} \in \mathcal{X} .
$$

Observe that $\mu \in \Delta(\mathcal{X})$. (If $\omega(n)=1$ for all $n \in \mathcal{N}$, then $\mu$ is an 'anonymous' profile; we do not generally assume anonymity.)

Judgement aggregation is the problem of selecting the element from $\mathcal{X}$ which best represents the 'collective will' of the voters described by profile $\mu$. Versions of this problem were studied by Guilbaud (1952), Wilson (1975), Rubinstein and Fishburn (1986), and Barthélémy and Janowitz (1991). Since the work of List and Pettit (2002), there has been much interest in this area; see List and Puppe (2009) for a recent survey.

Fix a set $\mathcal{K}$ and a judgement space $\mathcal{X} \subseteq\{ \pm 1\}^{\mathcal{K}}$. A judgement aggregation rule on $\mathcal{X}$ is a multifunction $F: \Delta(\mathcal{X}) \rightrightarrows \mathcal{X}$; for any $\mu \in \Delta(\mathcal{X})$, it yields a nonempty (usually singleton) subset $F(\mu) \subseteq \mathcal{X}$, which represents the social consensus given the profile $\mathbf{x}$. Sometimes we restrict $F$ to a smaller domain. For example, if $\omega: \mathcal{N} \longrightarrow \mathbb{R}_{+}$is a weight function, let $\Delta_{\omega}(\mathcal{X})$ be the set of all profiles obtained as in equation (1) for some assignment $\left\{\mathbf{y}^{n}\right\}_{n \in \mathcal{N}} \subset \mathcal{X}$ of opinions to the individual voters. We shall sometimes consider a rule $F_{\omega}: \Delta_{\omega}(\mathcal{X}) \rightrightarrows \mathcal{X}$.

On the other hand, we sometimes define $F$ over a larger domain. For example, let $\mathfrak{X}$ be a collection of judgement spaces (possibly with varying choices of $\mathcal{K}$ ). Let $\Delta(\mathfrak{X})$ be the set of all ordered pairs $(\mathcal{X}, \mu)$, where $\mathcal{X} \in \mathfrak{X}$ and $\mu \in \Delta(\mathcal{X})$. A (judgement) aggregation rule on $\mathfrak{X}$ is a multifunction $F: \Delta(\mathfrak{X}) \rightrightarrows \bigcup_{\mathcal{X} \in \mathfrak{X}} \mathcal{X}$ such that, for each $\mathcal{X} \in \mathfrak{X}$ and $\mu \in \Delta(\mathcal{X})$, we have $F(\mathcal{X}, \mu) \subseteq \mathcal{X}$. (Even in this case, we will indicate $F(\mathcal{X}, \mu)$ by " $F(\mu)$ " if $\mathcal{X}$ is clear from context.)

Condorcet admissibility. Treat $\{ \pm 1\}^{\mathcal{K}}$ as a subset of the vector space $\mathbb{R}^{\mathcal{K}}$. For any profile $\mu \in \Delta(\mathcal{X})$, we define the tally vector $\widetilde{\mu} \in \mathbb{R}^{\mathcal{K}}$ by

$$
\tilde{\mu}_{k}:=\sum_{\mathbf{x} \in \mathcal{X}} x_{k} \mu(\mathbf{x}), \quad \text { for all } k \in \mathcal{K} .
$$

Thus, $\widetilde{\mu} \in \operatorname{conv}(\mathcal{X})$ (the convex hull of $\mathcal{X}$ in $\mathbb{R}^{\mathcal{K}}$ ). The vector $\widetilde{\mu}$ tallies how much 'voter support' there is for each of the propositions in $\mathcal{K}$. For any $k \in \mathcal{K}$, we have $\widetilde{\mu}_{k}>0$ (resp. 
$<0$ ) if a majority asserts (resp. denies) proposition $k$, and $\widetilde{\mu}_{k}=1$ (resp. -1 ) if the voters unanimously assert (resp. deny) proposition $k$. For any $\mathbf{x} \in\{ \pm 1\}^{\mathcal{K}}$, let

$$
\mathcal{M}(\mu, \mathbf{x}) \quad:=\left\{k \in \mathcal{K} ; x_{k} \widetilde{\mu}_{k} \geq 0\right\} .
$$

This is the set of all propositions where $\mathbf{x}$ agrees with the majority view. Let $\operatorname{Maj}(\mu):=$ $\left\{\mathbf{x} \in\{ \pm 1\}^{\mathcal{K}} ; \mathcal{M}(\mu, \mathbf{x})=\mathcal{K}\right\}$. This set is always nonempty, and is usually a singleton, unless there is a 'perfect tie' on some propositions. (If $\operatorname{Maj}(\mu)=\{\mathbf{x}\}$, then we will abuse notation by writing "Maj $(\mu)=\mathbf{x}$ " and defining $\operatorname{Maj}_{k}(\mathbf{x}):=x_{k}$ for all $k \in \mathcal{K}$.)

If $\operatorname{Maj}(\mu) \cap \mathcal{X} \neq \emptyset$, then it is possible to comply with the majority opinion on every proposition, while still respecting the logical constraints defining $\mathcal{X}$. Unfortunately, this is generally not the case - often $\operatorname{Maj}(\mu) \cap \mathcal{X}=\emptyset$. (In the case of aggregation over $\mathcal{X}_{\mathcal{A}}^{\text {pr }}$ with $|\mathcal{A}| \geq 3$, this problem was first observed by Condorcet (1785).)

We say that $\mathbf{x}$ is Condorcet admissible if $\mathcal{M}(\mu, \mathbf{x})$ is maximal in $\mathcal{X}$-that is, there exists no $\mathbf{y} \in \mathcal{X}$ such that $\mathcal{M}(\mu, \mathbf{x}) \subsetneq \mathcal{M}(\mu, \mathbf{y})$. Let Cond $(\mathcal{X}, \mu)$ be the set of all Condorcet admissible elements. This set is always nonempty, and its elements are the "best approximations' of $\operatorname{Maj}(\mu)$ which are feasible given the logical constraints of $\mathcal{X}$. Thus, a 'majoritarian' judgement aggregation rule should always select from Cond $(\mathcal{X}, \mu)$. Unfortunately, for many judgement aggregation problems, the set Cond $(\mathcal{X}, \mu)$ is quite huge —indeed, in some cases Cond $(\mathcal{X}, \mu)=\mathcal{X}$; see Nehring et al. (2011). Thus, it is necessary to refine the notion of Condorcet admissibility in some way.

Supermajoritarian efficiency. Let $\mu \in \Delta(\mathcal{X})$ and let $q \in[0,1]$. For any $\mathbf{x} \in \mathcal{X}$, let

$$
\gamma_{\mu, \mathbf{x}}(q) \quad:=\quad \#\left\{k \in \mathcal{K} ; x_{k} \widetilde{\mu}_{k} \geq q\right\} .
$$

This measures the number of coordinates of $\mathbf{x}$ for which the popular support exceeds the supermajoritarian threshold $q$. For example, $\gamma_{\mu, \mathbf{x}}(0)$ is the number of coordinates where $\mathbf{x}$ receives a bare majority, $\gamma_{\mu, \mathbf{x}}(0.5)$ is the number of coordinates where $\mathbf{x}$ receives a $75 \%$ supermajority, and $\gamma_{\mu, \mathbf{x}}(1)$ is the number of coordinates where $\mathbf{x}$ receives unanimous support.

For any $\mathbf{x}, \mathbf{y} \in \mathcal{X}$, write " $\mathbf{x} \underset{\mu}{\triangleright} \mathbf{y}$ " if $\gamma_{\mu, \mathbf{x}}(q) \geq \gamma_{\mu, \mathbf{y}}(q)$ for all $q \in(0,1]$, with at least one strict inequality. Then $\triangleright_{\mu}$ is a partial order on $\mathcal{X}$. An element $\mathrm{x} \in \mathcal{X}$ is supermajoritarian efficient (SME) if $\mathbf{x}$ is undominated in the poset $(\mathcal{X}, \underset{\mu}{\triangleright})$. This means that it is impossible to change some coordinates of $\mathbf{x}$ to capture one more supermajority of size $q$, without losing some other supermajority of size $q^{\prime} \geq q$. This refinement of Condorcet admissibility is based on the idea that it is acceptable for the aggregation rule $F$ to overrule a supermajority in one coordinate, but only if this allows $F$ to agree with a larger supermajority in some other coordinate.

Let $\operatorname{SME}(\mathcal{X}, \mu)$ be the set of SME elements in $\mathcal{X}$. An aggregation rule $F: \Delta(\mathcal{X}) \rightrightarrows \mathcal{X}$ is supermajoritarian efficient if $F(\mu) \subseteq \operatorname{SME}(\mathcal{X}, \mu)$ for all $\mu \in \Delta(\mathcal{X})$. For example, the median rule is defined:

$$
\begin{aligned}
& \operatorname{Median}(\mathcal{X}, \mu):=\underset{\mathbf{x} \in \mathcal{X}}{\operatorname{argmax}}(\mathbf{x} \bullet \widetilde{\mu}), \quad \text { for all } \mu \in \Delta(\mathcal{X}), \\
& \text { where } \mathbf{x} \bullet \widetilde{\mu}:=\sum_{k \in \mathcal{K}} x_{k} \widetilde{\mu}_{k}, \quad \text { for any } \mathbf{x} \in \mathcal{X} .
\end{aligned}
$$


In the setting of Arrovian preference aggregation (i.e. when $\mathcal{X}$ is a permutahedron), this corresponds to the Kemeny (1959) rule, which has been analyzed by Young and Levenglick (1978) and Young $(1986,1988,1995,1997)$. As a general-purpose judgement aggregation rule, the median rule has been studied by Barthélémy and Monjardet $(1981,1988)$, Barthélémy (1989) and Barthélémy and Janowitz (1991).

A (real-valued) gain function is an increasing function $\phi:[-1,1] \longrightarrow \mathbb{R}$. For any judgement space $\mathcal{X} \subseteq\{ \pm 1\}^{\mathcal{K}}$ and any gain function $\phi$, we define the additive support rule $F_{\phi}: \Delta(\mathcal{X}) \rightrightarrows \mathcal{X}$ as follows:

$$
\text { for all } \mu \in \Delta(\mathcal{X}), \quad F_{\phi}(\mu):=\underset{\mathbf{x} \in \mathcal{X}}{\operatorname{argmax}}\left(\sum_{k \in \mathcal{K}} \phi\left(x_{k} \widetilde{\mu}_{k}\right)\right) .
$$

For example, fix $s>0$, and suppose $\phi(r)=s r$ for all $r \in[-1,1]$; then $F_{\phi}$ is the median rule. More generally, fix $d \in(0, \infty)$, and define $\phi^{d}:[-1,1] \longrightarrow \mathbb{R}$ by $\phi^{d}(r):=\operatorname{sign}(r) \cdot|r|^{d}$ for all $r \in[-1,1]$. (If $d$ was an odd integer, we could simply write $\phi^{d}(r):=r^{d}$.) The corresponding additive support rule $H^{d}:=F_{\phi^{d}}$ is called the homogeneous rule of degree $d$. If $d=1$, then $H^{d}$ is the median rule. If $0<d<1$, then the function $\phi^{d}$ is 'S-shaped'; this means that $H^{d}$ magnifies the distinction between majorities and minorities, but diminishes the distinction between small supermajorities and large ones. On the other hand, if $d>1$, then the function $\phi^{d}$ is 'inverse S-shaped'; thus, $H^{d}$ diminishes the distinction between majorities and minorities, but magnifies the distinction between small supermajorities and large ones. As $d \rightarrow \infty$ it is increasingly the case that one large supermajority (especially a unanimous one) can overrule many small supermajorities.

Any additive support rule is SME (see Proposition 1.1(b) below). Another SME rule is the LexiMin rule. For any $\mathbf{x}, \mathbf{y} \in \mathcal{X}$, write " $\mathbf{x} \underset{\mu}{\widetilde{y}} \mathbf{~ " ~ i f ~} \gamma_{\mu, \mathbf{x}}=\gamma_{\mu, \mathbf{y}}$; otherwise write "x $\underset{\mu}{\prec} \mathbf{y}$ " if there exists some $Q \in(0,1]$ such that $\gamma_{\mu, \mathbf{x}}(q)=\gamma_{\mu, \mathbf{y}}(q)$ for all $q>Q$, while $\gamma_{\mu, \mathbf{x}}(q)<\gamma_{\mu, \mathbf{y}}(q)$. Then $\underset{\mu}{\prec}$ is a complete, transitive ordering of $\mathcal{X}$. We then define

$$
\operatorname{LexiMin}(\mathcal{X}, \mu):=\max (\mathcal{X}, \underset{\mu}{\prec}) .
$$

In other words, LexiMin first maximizes the number of coordinates which receive unanimous support (if any); then, for every possible supermajoritarian threshold $q \in(0,1]$, LexiMin maximizes the number of coordinates where the support exceeds $q$, with higher values of $q$ given lexicographical priority over lower ones. This rule was proposed by Tideman (1987) in the setting of preference aggregation (he called it the ranked pairs rule; see also Zavist and Tideman (1989)). The leximin rule can be seen as the 'limit' of the system $\left\{H^{d}\right\}_{d>0}$ of homogeneous rules as $d \rightarrow \infty$ (Nehring and Pivato, 2011b).

Strong supermajoritarian efficiency. For any probability measure $\rho \in \Delta(\mathcal{X})$, and any $q \in(0,1]$, define

$$
\gamma_{\mu, \rho}(q):=\sum_{\mathbf{x} \in \mathcal{X}} \rho(\mathbf{x}) \cdot \gamma_{\mu, \mathbf{x}}(q)
$$

Intuitively, suppose $\rho$ describes the outcome of a 'stochastic' judgement aggregation rule, which produces a $\rho$-random element of $\mathcal{X}$ as output. Thus, $\gamma_{\mu, \rho}(q)$ is the expected value of 
$\gamma_{\mu, \boldsymbol{\bullet}}(q)$ for this stochastic rule. For any $\mathbf{x} \in \mathcal{X}$, define $\delta_{\mathbf{x}} \in \Delta(\mathcal{X})$ by

$$
\delta_{\mathbf{x}}(\mathbf{x}):=1, \quad \text { and } \quad \delta_{\mathbf{x}}(\mathbf{y}):=0, \quad \text { for all } \mathbf{y} \in \mathcal{X} \backslash\{\mathbf{x}\} .
$$

Clearly $\gamma_{\mu, \delta_{\mathbf{x}}}=\gamma_{\mu, \mathbf{x}}$. For any $\rho_{1}, \rho_{2} \in \Delta(\mathcal{X})$, write " $\rho_{1} \stackrel{\triangleright}{\mu}_{\rho} \rho_{2}$ " if $\gamma_{\mu, \rho_{1}}(q) \geq \gamma_{\mu, \rho_{2}}(q)$ for all

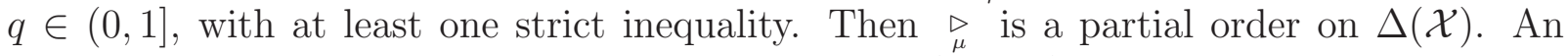
element $\mathrm{x} \in \mathcal{X}$ is strongly supermajoritarian efficient (SSME) if $\delta_{\mathbf{x}}$ is undominated in the poset $\left(\Delta(\mathcal{X}),{ }_{\mu}^{\triangleright}\right)$. Let $\operatorname{SSME}(\mathcal{X}, \mu)$ be the set of $\operatorname{SSME}$ elements in $\mathcal{X}$. An aggregation rule $F$ is strongly supermajoritarian efficient if $F(\mathcal{X}, \mu) \subseteq \operatorname{SSME}(\mathcal{X}, \mu)$ for all $\mathcal{X}$ and $\mu$.

Proposition 1.1 Let $(\mathcal{K}, \mathcal{X}, \mu)$ be a judgement aggregation problem.

(a) $\emptyset \neq \operatorname{SSME}(\mathcal{X}, \mu) \subseteq \operatorname{SME}(\mathcal{X}, \mu) \subseteq \operatorname{Cond}(\mathcal{X}, \mu)$. In general, these inclusions are strict.

If $\operatorname{Maj}(\mu) \cap \mathcal{X} \neq \emptyset$, then $\operatorname{SSME}(\mathcal{X}, \mu)=\operatorname{SME}(\mathcal{X}, \mu)=\operatorname{Cond}(\mathcal{X}, \mu)=\operatorname{Maj}(\mu) \cap \mathcal{X}$.

(b) If $\phi$ any gain function, then the additive support rule $F_{\phi}$ is strongly supermajoritarian efficient. In fact, if $\Phi_{I}$ is the set of all odd, continuous, real-valued gain functions, then $\bigcup_{\phi \in \Phi_{I}} F_{\phi}(\mu)=\operatorname{SSME}(\mathcal{X}, \mu)$.

This paper studies the structure and properties of supermajoritarian efficient judgement aggregation rules, and provides representation theorems and axiomatic characterizations for several classes of such rules. One of our goals is to provide a ready-to-use 'menu' of supermajoritarian efficient aggregation rules. An end-user seeking a rule with particular properties can choose the appropriate item from this menu.

This paper is organized as follows. In $\S 2$ we show that the set $\operatorname{SME}(\mathcal{X}, \mu)$ is generically a singleton whenever $\mathcal{X}$ satisfies a geometric condition called 'proximality'. Proximality holds whenever $|\mathcal{K}|=3$ (e.g. it holds for $\mathcal{X}_{\mathcal{A}}^{\mathrm{pr}}$ when $|\mathcal{A}|=3$ ). It also holds for certain 'committee selection' problems. In $\S 3$ we introduce a more general class of additive support rules (by allowing $\phi$ to range over a hyperreal field) and study some of their properties. We introduce a time-consistency condition called separability; the first main result of this paper states that a judgement aggregation rule $F$ is SME and separable if and only if $F$ can be represented as an additive support rule $F_{\phi}$.

Section 4 contains our second main result, which states that the median rule is the only regular, upper hemicontinuous, additive support rule satisfying a condition called reinforcement: if two sub-populations of voters both select a certain judgement $\mathrm{x} \in \mathcal{X}$, then the combined population of voters should also select $\mathbf{x}$. This is analogous to the classic result of Young and Levenglick (1978) characterizing the Kemeny rule. In $\S 5$, we show that the set of homogeneous rules are the only regular, upper hemicontinuous, additive support rules satisfying neutral reinforcement. This means that, for any $\mathbf{x}, \mathbf{y} \in \mathcal{X}$, if one sub-population selects the judgements $\{\mathbf{x}, \mathbf{y}\}$, and the other population is evenly split between $\mathbf{x}$-supporters and $\mathbf{y}$-supporters, then the combined population should also select $\{\mathbf{x}, \mathbf{y}\}$. Finally, in $\S 6$ we consider what conditions under which the gain function $\phi$ is continuous and/or unique up to scalar multiplication. Appendices A-D contain background and technical results. Appendix E contains the proofs of all the main results. 


\section{Proximality and supermajoritarian determinacy}

A judgement space $\mathcal{X}$ is supermajoritarian determinate if, for any $\mu \in \Delta(\mathcal{X})$, and any $\mathbf{x}, \mathbf{y} \in \operatorname{SME}(\mathcal{X}, \mu)$, we have $\gamma_{\mu, \mathbf{x}}=\gamma_{\mu, \mathbf{y}}$. This means that all additive rules yield the same output: $F_{\phi}(\mathcal{X}, \mu)=\operatorname{SME}(\mathcal{X}, \mu)$ for all nondecreasing $\phi:[-1,1] \longrightarrow \mathbb{R}$. Also, generically, $\operatorname{SME}(\mathcal{X}, \mu)$ is a singleton. Which judgement spaces are supermajoritarian determinate?

Let $\mathcal{C}:=\operatorname{conv}(\mathcal{X})$ (a compact, convex polyhedron in $\mathbb{R}^{\mathcal{K}}$ ). Thus, $\widetilde{\mu} \in \mathcal{C}$ for any $\mu \in \Delta(\mathcal{X})$. Let $D:=\operatorname{dim}[\mathcal{C}]$. For any $\mathbf{x}, \mathbf{y} \in \mathcal{X}$, let $\mathcal{B}_{\mathbf{x}, \mathbf{y}}:=\{\mathbf{c} \in \mathcal{C} ; \operatorname{Median}(\mathbf{c}) \supseteq\{\mathbf{x}, \mathbf{y}\}\}$. Equivalently, $\mathcal{B}_{\mathbf{x}, \mathbf{y}}:=\{\mathbf{c} \in \mathcal{C} ; \mathbf{x} \bullet \mathbf{c}=\mathbf{y} \bullet \mathbf{c} \geq \mathbf{z} \bullet \mathbf{c}$, for all $\mathbf{z} \in \mathcal{X}\}$. Thus, $\mathcal{B}_{\mathbf{x}, \mathbf{y}}$ is a convex sub-polyhedron of $\mathcal{C}$ (defined by a finite system of linear (in)equalities). Write " $\mathbf{x} \underset{\overline{\mathcal{X}}}{\mathbf{y}}$ " if $\operatorname{dim}\left(\mathcal{B}_{\mathbf{x}, \mathbf{y}}\right)=D-1$. The relation " $\overline{\bar{X}}$ " defines a graph on $\mathcal{X}$, which we will call the internal graph.

For any $\mathbf{x}, \mathbf{y} \in \mathcal{X}$, let $\mathcal{K}_{ \pm}(\mathbf{x}, \mathbf{y}):=\left\{k \in \mathcal{K} ; x_{k} \neq y_{k}\right\}$. Let $d(\mathbf{x}, \mathbf{y}):=\left|\mathcal{K}_{ \pm}(\mathbf{x}, \mathbf{y})\right|$ be the Hamming distance from $\mathbf{x}$ to $\mathbf{y}$. Say $\mathcal{X}$ is proximal if $d(\mathbf{x}, \mathbf{y}) \leq 2$ for all $\mathbf{x} \overline{\overline{\mathcal{X}}} \mathbf{y} \in \mathcal{X}$. Finally, say $\mathcal{X}$ is thick if $\operatorname{dim}[\operatorname{conv}(\mathcal{X})]=K$ - that is, $\operatorname{int}(\operatorname{conv}(\mathcal{X})) \neq \emptyset$. This means that no coordinate of $\mathcal{X}$ can be expressed as an affine function of other coordinates. Thickness is a very mild condition which is satisfied by most 'interesting' judgement spaces.

Theorem $2.1 \quad$ (a) If $\mathcal{X}$ is proximal, then $\mathcal{X}$ is supermajoritarian determinate.

(b) If $\mathcal{X}$ is thick and supermajoritarian determinate, then $\mathcal{X}$ is proximal.

Example 2.2. If $|\mathcal{K}|=3$, then any $\mathcal{X} \subseteq\{ \pm 1\}^{\mathcal{K}}$ is proximal, and thus, supermajoritarian determinate. To see this, observe that $d(\mathbf{x}, \mathbf{y}) \leq 3$ for all $\mathbf{x}, \mathbf{y} \in\{ \pm 1\}^{\mathcal{K}}$, and $d(\mathbf{x}, \mathbf{y})=3$ if and only if $\mathbf{x}=-\mathbf{y}$, in which case clearly $\mathbf{x}$ 寿 $\mathbf{y}$.

In particular, if $|\mathcal{A}|=3$, then $\mathcal{X}_{\mathcal{A}}^{\mathrm{pr}}$ is proximal, hence supermajoritarian determinate.

For another example of proximality, let $\mathcal{K}$ represent a set of 'candidates'. Any $\mathbf{x} \in$ $\{ \pm 1\}^{\mathcal{K}}$ represents a 'committee' (where $x_{k}=1$ if and only if candidate $k$ is on the committee). Let $|\mathbf{x}|:=\#\left\{k \in \mathcal{K} ; x_{k}=1\right\}$ be the size of this committee. Let $0 \leq I \leq J \leq K$, and let $\mathcal{X}^{\text {com }}:=\left\{\mathbf{x} \in\{ \pm 1\}^{\mathcal{K}} ; I \leq|\mathbf{x}| \leq J\right\}$. Thus, $\mathcal{X}^{\text {com }}$ represents the set of committees drawn from $\mathcal{K}$, containing at least $I$ members and at most $J$ members.

Proposition 2.3 $\mathcal{X}^{\mathrm{com}}$ is proximal, and thus, supermajoritarian determinate.

\section{Separability and additive support rules}

We will now show that the class of additive support rules is characterized by supermajoritarian efficiency along with a simple consistency condition involving multiple judgement spaces. Let $\mathcal{K}_{1}$ and $\mathcal{K}_{2}$ be disjoint sets, and let $\mathcal{K}:=\mathcal{K}_{1} \sqcup \mathcal{K}_{2}$. Let $\mathcal{X}_{1} \subseteq\{ \pm 1\}^{\mathcal{K}_{1}}$, let $\mathcal{X}_{2} \subseteq\{ \pm 1\}^{\mathcal{K}_{2}}$ and let $\mathcal{X}:=\mathcal{X}_{1} \times \mathcal{X}_{2} \subseteq\{ \pm 1\}^{\mathcal{K}}$. If $\mathbf{x} \in \mathcal{X}$, then we write $\mathbf{x}=\left(\mathbf{x}_{1}, \mathbf{x}_{2}\right)$ where $\mathbf{x}_{n} \in \mathcal{X}_{n}$ for $n=1,2$. For any $\mu \in \Delta(\mathcal{X})$, let $\mu^{(1)} \in \Delta\left(\mathcal{X}_{1}\right)$ be the marginal profile of $\mu$ on $\mathcal{X}_{1}$. That is:

$$
\text { for all } \mathbf{x}_{1} \in \mathcal{X}_{1}, \quad \mu^{(1)}\left(\mathbf{x}_{1}\right):=\sum_{\mathbf{x}_{2} \in \mathcal{X}_{2}} \mu\left(\mathbf{x}_{1}, \mathbf{x}_{2}\right) \text {. }
$$


Likewise, define $\mu^{(2)} \in \Delta\left(\mathcal{X}_{2}\right)$. (Observe that $\widetilde{\mu}=\left(\widetilde{\mu}^{(1)}, \widetilde{\mu}^{(2)}\right)$, because $\widetilde{\mu}_{k}^{(1)}=\widetilde{\mu}_{k}$ for all $k \in \mathcal{K}_{1}$ and $\widetilde{\mu}_{k}^{(2)}=\widetilde{\mu}_{k}$ for all $k \in \mathcal{K}_{2}$.)

For any judgement space $\mathcal{X}$, and any $n \in \mathbb{N}$, let $\mathcal{X}^{n}:=\mathcal{X} \times \mathcal{X} \times \cdots \times \mathcal{X}$ be the $n$-fold Cartesian product of $\mathcal{X}$. Let $\langle\mathcal{X}\rangle:=\left\{\mathcal{X}^{n} ; n \in \mathbb{N}\right\}$. Let $F: \Delta(\mathcal{X}) \rightrightarrows \mathcal{X}$ be a judgement aggregation rule. For all $n \in \mathbb{N}$, define the rule $F^{n}: \Delta\left(\mathcal{X}^{n}\right) \rightrightarrows \mathcal{X}^{n}$ as follows:

$$
F^{n}(\mu):=F\left(\mu^{(1)}\right) \times F\left(\mu^{(2)}\right) \times \cdots \times F\left(\mu^{(n)}\right), \quad \text { for all } \mu \in \Delta\left(\mathcal{X}^{n}\right) .
$$

Here, $\mu^{(1)}, \ldots, \mu^{(n)} \in \Delta(\mathcal{X})$ are the marginal profiles of $\mu$ onto the $n$ copies of $\mathcal{X}$ which comprise $\mathcal{X}^{n}$. If we define $\Delta\langle\mathcal{X}\rangle:=\bigcup_{n=1}^{\infty} \Delta\left(\mathcal{X}^{n}\right)$, then we obtain a judgement aggregation rule $F^{*}: \Delta\langle\mathcal{X}\rangle \rightrightarrows \bigcup_{n=1}^{\infty} \mathcal{X}^{n}$. We say that $F^{*}$ is supermajoritarian efficient if $F^{n}$ is supermajoritarian efficient on $\Delta\left(\mathcal{X}^{n}\right)$ for all $n \in \mathbb{N}$.

Fix $W \in \mathbb{N}$. Let $\Delta_{W}\langle\mathcal{X}\rangle$ be the set of all profiles on $\langle\mathcal{X}\rangle$ involving exactly $W$ equally weighted (i.e. 'anonymous') voters. Thus, if $\mathcal{Q}_{W}:=\left\{1-\frac{2 k}{W} ; k \in[0 \ldots W]\right\}$, then $\mathcal{Q}_{W}=$ $\left\{\widetilde{\mu}_{k} ; k \in \mathcal{K}\right.$ and $\left.\mu \in \Delta_{W}\langle\mathcal{X}\rangle\right\}$. If $\mu \in \Delta_{W}\left(\mathcal{X}^{n}\right)$, then $\mu^{(1)}, \ldots, \mu^{(n)} \in \Delta_{W}(\mathcal{X})$. Thus, as long as $F$ is well-defined on $\Delta_{W}(\mathcal{X})$, the extended rule $F^{*}$ is well-defined on $\Delta_{W}\langle\mathcal{X}\rangle$. We say that $F^{*}$ is supermajoritarian efficient on $\Delta_{W}\langle\mathcal{X}\rangle$ if $F^{n}$ is supermajoritarian efficient on $\Delta_{W}\left(\mathcal{X}^{n}\right)$ for all $n \in \mathbb{N}$.

Theorem 3.1 Let $W \in \mathbb{N}$, let $\mathcal{X}$ be a judgement space, and let $F: \Delta_{W}(\mathcal{X}) \rightrightarrows \mathcal{X}$ be a judgement aggregation rule.

The rule $F^{*}$ is SME on $\Delta_{W}\langle\mathcal{X}\rangle$ if and only if there is an increasing function $\phi_{W}$ : $\mathcal{Q}_{W} \longrightarrow \mathbb{R}$ such that $F^{n}(\mu) \subseteq F_{\phi_{W}}\left(\mathcal{X}^{n}, \mu\right)$ for all $n \in \mathbb{N}$ and $\mu \in \Delta_{W}\left(\mathcal{X}^{n}\right)$.

If $W$ is fixed in advance, and all voters are anonymous, then Theorem 3.1 is sufficient for most practical purposes. However, if $W$ or the weights of individual voters are allowed to vary, then the rule $F$ must be well-defined on all of $\Delta(\mathcal{X})$. Also, Theorem 3.1 only states that $F^{*}$ is contained in $F_{\phi_{W}}$; if we want to ensure that $F^{*}=F_{\phi_{W}}$, we need a continuity condition which only makes sense if $F$ is defined on all of $\Delta(\mathcal{X})$.

To extend Theorem 3.1 this setting, we must extend the range of $\phi$ to hyperreal numbers. Loosely speaking, a hyperreal number field is a linearly ordered field ${ }^{*} \mathbb{R}$ which contains the field $\mathbb{R}$ as a proper subfield, but which also contains a large number of 'infinite' and 'infinitesimal' elements, having a well-defined arithmetic. (Formally, ${ }^{*} \mathbb{R}$ is an ultrapower of $\mathbb{R}$; see Appendix A for details.) A (hyperreal) gain function is now any increasing function $\phi:[-1,1] \longrightarrow * \mathbb{R}$. (Note that a real-valued gain function is a special case, because $\mathbb{R} \subset{ }^{*} \mathbb{R}$.) Given any hyperreal gain function $\phi$, we define the additive support rule $F_{\phi}$ as in eqn.(6).

For example, let ${ }^{*} d \in{ }^{*} \mathbb{R}$ be any positive infinite ${ }^{1}$ hyperreal number, and define $\phi^{*} d$ : $[-1,1] \longrightarrow{ }^{*} \mathbb{R}$ by $\phi^{*} d(r):=\operatorname{sign}(r) \cdot|r|^{*} d$. Then Nehring and Pivato (2011b) show that the additive support rule $F_{\phi^{*}}$ is the leximin rule introduced in $\S 1$.

If ${ }^{*} r \in{ }^{*} \mathbb{R}$ and $x \in\{ \pm 1\}$, then $x \cdot{ }^{*} r$ is also an element of ${ }^{*} \mathbb{R}$. Thus, if ${ }^{*} \mathrm{r} \in{ }^{*} \mathbb{R}^{\mathcal{K}}$ and $\mathbf{x} \in\{ \pm 1\}^{\mathcal{K}}$, then we can define $\mathbf{x} \bullet{ }^{*} \mathbf{r}:=\sum_{k \in \mathcal{K}} x_{k} \cdot{ }^{*} r_{k}$, an element of ${ }^{*} \mathbb{R}$. In particular, for any $\mu \in \Delta(\mathcal{X})$, we define $\phi(\widetilde{\mu}):=\left[\phi\left(\widetilde{\mu}_{k}\right)\right]_{k \in \mathcal{K}} \in{ }^{*} \mathbb{R}^{\mathcal{K}}$; then $\mathbf{x} \bullet \phi(\widetilde{\mu})=\sum_{k \in \mathcal{K}} x_{k} \cdot \phi\left(\widetilde{\mu}_{k}\right)$. The function $\phi$ is odd if $\phi(-r)=-\phi(r)$ for all $r \in[-1,1]$. We can assume without loss of generality that $\phi$ is odd, which yields a convenient expression for $F_{\phi}(\mathcal{X}, \mu)$ :

\footnotetext{
${ }^{1}$ A positive hyperreal number ${ }^{*} d$ is infinite if ${ }^{*} d>M$ for every $M \in \mathbb{N}$.
} 
Lemma 3.2 Let $\phi:[-1,1] \longrightarrow{ }^{*} \mathbb{R}$ be any gain function.

(a) There exists an odd function $\widehat{\phi}:[-1,1] \longrightarrow{ }^{*} \mathbb{R}$ such that, for all judgement spaces $\mathcal{X}$ and all $\mu \in \Delta(\mathcal{X})$, we have $F_{\phi}(\mathcal{X}, \mu)=F_{\widehat{\phi}}(\mathcal{X}, \mu)$.

(b) If $\phi$ is odd, then for all judgement spaces $\mathcal{X}$ and all $\mu \in \Delta(\mathcal{X})$, we have $F_{\phi}(\mathcal{X}, \mu)=$ $\underset{\mathbf{x} \in \mathcal{X}}{\operatorname{argmax}} \mathbf{x} \bullet \phi(\widetilde{\mu})$.

A judgement aggregation rule $F: \Delta(\mathcal{X}) \rightrightarrows \mathcal{X}$ is upper hemicontinuous (UHC) if, for every $\mu \in \Delta(\mathcal{X})$, each of the following two equivalent statements is true:

(a) There exists some $\bar{\epsilon}>0$ such that, for any $\epsilon \in(0, \bar{\epsilon})$ any other $\nu \in \Delta(\mathcal{X})$, we have $F(\epsilon \nu+(1-\epsilon) \mu) \subseteq F(\mu)$.

(b) For every sequence $\left\{\mu_{n}\right\}_{n=1}^{\infty} \subset \Delta(\mathcal{X})$, and every $\mathbf{x} \in \mathcal{X}$, if $\lim _{n \rightarrow \infty} \mu_{n}=\mu$, and $\mathbf{x} \in F\left(\mu_{n}\right)$ for all $n \in \mathbb{N}$, then $\mathbf{x} \in F(\mu)$.

Condition (a) is sometimes described as the 'overwhelming majority' property. Heuristically, the profile $\epsilon \nu+(1-\epsilon) \mu$ represents a mixture of two populations: a small 'minority' described by the profile $\nu$, and a large 'majority' represented by the profile $\mu$. Condition (a) says that, if the majority is large enough, then its views effectively overwhelm the minority view (except that the minority can perhaps act as a 'tie-breaker' in some cases). Condition (b) means that the outcome of judgement aggregation is robust under small measurement errors or perturbations of public opinion. ${ }^{2}$ We now come to the extended version of Theorem 3.1:

Theorem 3.3 Let $\mathcal{X}$ be a judgement space, and let $F: \Delta(\mathcal{X}) \rightrightarrows \mathcal{X}$ be an upper hemicontinuous judgement aggregation rule.

The rule $F^{*}$ is supermajoritarian efficient on $\Delta\langle\mathcal{X}\rangle$ if and only if there is an hyperreal field ${ }^{*} \mathbb{R}$ and a gain function $\phi:[-1,1] \longrightarrow{ }^{*} \mathbb{R}$ such that $F^{n}(\mu)=F_{\phi}\left(\mathcal{X}^{n}, \mu\right)$ for all $n \in \mathbb{N}$ and $\mu \in \Delta\left(\mathcal{X}^{n}\right)$.

In fact, Theorems 3.1 and 3.3 are both special cases of a more general result. A judgement monoid is a collection $\mathfrak{X}$ of judgement spaces which is closed under Cartesian products. That is: for any $\mathcal{X}, \mathcal{Y} \in \mathfrak{X}$, we also have $\mathcal{X} \times \mathcal{Y} \in \mathfrak{X}$. For example, $\langle\mathcal{X}\rangle$ is a monoid, and the set of all judgement spaces is a judgement monoid. Fix some judgement spaces $\mathcal{X}_{1}, \ldots, \mathcal{X}_{J}$ (for some $J \in \mathbb{N}$ ), then we call the set $\mathfrak{X}=\left\{\mathcal{X}_{1}^{m_{1}} \times \mathcal{X}_{2}^{m_{2}} \times \cdots \times \mathcal{X}_{J}^{m_{J}}\right.$; $\left.m_{1}, \ldots, m_{J} \in \mathbb{N}\right\}$ a finitely generated monoid.

We define $\Delta(\mathfrak{X}):=\bigcup_{\mathcal{X} \in \mathfrak{X}} \Delta(\mathcal{X})$. A judgement aggregation rule $F: \Delta(\mathfrak{X}) \rightrightarrows \bigcup_{\mathcal{X} \in \mathfrak{X}} \mathcal{X}$ is separable if, for all $\mathcal{X}_{1}, \mathcal{X}_{2} \in \mathfrak{X}$ and all $\mu \in \Delta\left(\mathcal{X}_{1} \times \mathcal{X}_{2}\right)$, we have $F\left(\mathcal{X}_{1} \times \mathcal{X}_{2}, \mu\right)=$ $F\left(\mathcal{X}_{1}, \mu^{(1)}\right) \times F\left(\mathcal{X}_{2}, \mu^{(2)}\right)$ (here, $\mu^{(1)} \in \Delta\left(\mathcal{X}_{1}\right)$ and $\mu^{(2)} \in \Delta\left(\mathcal{X}_{2}\right)$ are the marginal profiles of $\mu$ ). For example: if $F: \Delta(\mathcal{X}) \rightrightarrows \mathcal{X}$ is a judgement aggregation rule, then the rule $F^{*}$ defined by formula (11) is the unique extension of $F$ to a separable rule on $\Delta\langle\mathcal{X}\rangle$.

\footnotetext{
${ }^{2}$ Note that a nontrivial judgement aggregation rule can never be lower hemicontinuous (because it is a nonconstant function from $\Delta(\mathcal{X})$ into a discrete set).
} 
A finitary weight function is a function $\omega: \mathbb{N} \longrightarrow \mathbb{R}_{+}$such that the set $\operatorname{supp}(\omega):=\{n \in \mathbb{N}$; $\omega(n)>0\}$ is finite. (If $|\operatorname{supp}(\omega)|=N$, then $\omega$ represents a weight function for $N$ voters.) Let $\Omega$ be the set of all finitary weight functions. For any $\omega \in \Omega$, let $\Delta_{\omega}(\mathfrak{X})$ be the set of all profiles in $\Delta(\mathfrak{X})$ generated using $\omega$, in the sense of eqn.(1). Let $\mathcal{Q}_{\omega}:=\left\{\widetilde{\mu}_{k} ; \mu \in \Delta_{\omega}(\mathfrak{X})\right.$ and $k \in \mathcal{K}\}$. (For example, suppose $W \in \mathbb{N}$, and $\omega(n)=1$ for all $n \in[1 \ldots W]$ while $\omega(0)=1$ for all $n>W$; then $\Delta_{\omega}\langle\mathcal{X}\rangle=\Delta_{W}\langle\mathcal{X}\rangle$ and $\mathcal{Q}_{\omega}=\mathcal{Q}_{W}$, as defined prior to Theorem 3.1.) Here is the main result of this section, and one of the two main results of the paper:

Theorem 3.4 Let $\mathfrak{X}$ be any judgement monoid, and let $F$ be a separable judgement aggregation rule on $\mathfrak{X}$.

(a) Let $\omega \in \Omega$ and suppose $\mathfrak{X}$ is finitely generated. The rule $F$ is $S M E$ on $\Delta_{\omega}(\mathfrak{X})$ if and only if there is a gain function $\phi_{\omega}: \mathcal{Q}_{\omega} \longrightarrow \mathbb{R}$ such that $F(\mathcal{X}, \mu) \subseteq F_{\phi_{\omega}}(\mathcal{X}, \mu)$ for all $\mathcal{X} \in \mathfrak{X}$ and $\mu \in \Delta_{\omega}(\mathcal{X})$.

(b) The rule $F$ is $S M E$ on $\Delta(\mathfrak{X})$ if and only if there is a hyperreal field * $\mathbb{R}$ and a gain function $\phi:[-1,1] \longrightarrow{ }^{*} \mathbb{R}$ such that $F(\mathcal{X}, \mu) \subseteq F_{\phi}(\mathcal{X}, \mu)$ for all $\mathcal{X} \in \mathfrak{X}$ and $\mu \in \Delta(\mathcal{X})$. In this case, for all $\mathcal{X} \in \mathfrak{X}$, there is a dense open subset $\mathcal{O} \subseteq \Delta(\mathcal{X})$ such that $F(\mathcal{X}, \mu)=F_{\phi}(\mathcal{X}, \mu)$ and is single-valued for all $\mu \in \mathcal{O}$.

(c) Let $F$ and $\phi$ be as in part (b). Fix $\mathcal{X} \in \mathfrak{X}$, and suppose $F$ is upper hemicontinuous on $\Delta(\mathcal{X})$. Then $F(\mathcal{X}, \mu)=F_{\phi}(\mathcal{X}, \mu)$ for all $\mu \in \Delta(\mathcal{X})$.

We finish this section by noting some other convenient properties of additive support rules. For any $\mu \in \Delta(\mathcal{X})$, let $\mathcal{X}(\mu):=\{\mathbf{x} \in \mathcal{X} ; \mu(\mathbf{x})>0\}$. Let $\mu^{\prime} \in \Delta(\mathcal{X})$ and let $\mathbf{y} \in \mathcal{X}$. We say that $\mu^{\prime}$ is more supportive than $\mu$ of $\mathbf{y}$ if $\mu^{\prime}(\mathbf{y})>\mu(\mathbf{y})$, while $\mu^{\prime}(\mathbf{x})<\mu(\mathbf{x})$ for all $\mathbf{x} \in \mathcal{X}(\mu) \backslash\{\mathbf{y}\}$, and $\mu^{\prime}(\mathbf{x})=\mu(\mathbf{x})=0$ for all $\mathbf{x} \in \mathcal{X} \backslash \mathcal{X}(\mu)$. For example: let $\delta_{\mathbf{y}} \in \Delta(\mathcal{X})$ be the 'point mass' at $\mathbf{y}\left(\right.$ so $\delta_{\mathbf{y}}(\mathbf{y}):=1$ and $\delta_{\mathbf{y}}(\mathbf{x}):=0$ for all $\left.\mathbf{x} \in \mathcal{X} \backslash\{\mathbf{y}\}\right)$. Then for any $\mu \in \Delta(\mathcal{X})$ and any $r \in(0,1]$, the convex combination $r \delta_{\mathbf{y}}+(1-r) \mu$ is more supportive than $\mu$ of $\mathbf{y}$. A judgement aggregation rule $F: \Delta(\mathcal{X}) \rightrightarrows \mathcal{X}$ is monotone if, for any $\mu, \mu^{\prime} \in \Delta(\mathcal{X})$, and $\mathbf{y} \in F(\mu)$, if $\mu^{\prime}$ is more supportive than $\mu$ of $\mathbf{y}$, then $F\left(\mu^{\prime}\right)=\{\mathbf{y}\}$. In other words: if $\mathbf{y}$ is already one of the winning alternatives, then any slight increase in the popular support for $\mathbf{y}$ at the expense of the support for other elements of $\mathcal{X}$ will make $\mathbf{y}$ the unique winning alternative. The rule $F$ is generically single-valued if there is an open dense subset $\mathcal{O} \subset \Delta(\mathcal{X})$ such that $F(\mu)$ is single-valued for all $\mu \in \mathcal{O}$.

Proposition 3.5 Let ${ }^{*} \mathbb{R}$ be any hyperreal field, and let $\phi:[-1,1] \longrightarrow{ }^{*} \mathbb{R}$ be any gain function. Then for any judgement space $\mathcal{X}$, the additive support rule $F_{\phi}$ is (a) strongly supermajoritarian efficient, (b) monotone, and (c) generically single-valued on $\Delta(\mathcal{X})$.

Nehring and Pivato (2011b) give a partial converse to this result: if $\phi$ is not strictly increasing, then there exists a judgement space $\mathcal{X}$ such that $F_{\phi}$ is not supermajoritarian efficient, generically single-valued, or monotone on $\Delta(\mathcal{X})$.

Proposition 3.6 Let $\phi:[-1,1] \longrightarrow \mathbb{R}$ be any continuous, real-valued gain function. Then for every judgement space $\mathcal{X}$, the rule $F_{\phi}$ is upper hemicontinuous on $\Delta(\mathcal{X})$. 
The next section provides a partial converse to this result, but the details are subtle. Nehring and Pivato (2011b) gives a different sort of partial converse to Proposition 3.6: if $\phi$ is not continuous, then there exists a judgement space $\mathcal{X}$ such that $F_{\phi}$ is not upper hemicontinous on $\Delta(\mathcal{X})$. Furthermore, Proposition 3.6 is true even for continuous hyperreal gain functions (under a suitable definition of 'continuity'). However, any such continuous hyperreal gain function is 'equivalent' to a real-valued gain function (Nehring and Pivato, 2011b). Thus, allowing $\phi$ to be hyperreal in Proposition 3.6 does not yield any additional generality.

\section{Reinforcement and the median rule}

Let $\mu_{1}, \mu_{2} \in \Delta(\mathcal{X})$ be two profiles, describing two subpopulations of size $S_{1}$ and $S_{2}$. Let $c_{1}=S_{1} /\left(S_{1}+S_{2}\right)$ and $c_{2}=S_{2} /\left(S_{1}+S_{2}\right)$. Then $\mu=c_{1} \mu_{1}+c_{2} \mu_{2}$ is the profile of the combined population. If each subpopulation separately endorses some position $\mathbf{x} \in \mathcal{X}$, then the combined population presumably should also endorse this position. A judgment aggregation rule $F: \Delta(\mathcal{X}) \rightrightarrows \mathcal{X}$ satisfies reinforcement if the following holds: for any $\mu_{1}, \mu_{2} \in \Delta(\mathcal{X})$ with $F\left(\mu_{1}\right) \cap F\left(\mu_{2}\right) \neq \emptyset$, and any $c_{1}, c_{2} \in(0,1)$ with $c_{1}+c_{2}=1$, if $\mu=c_{1} \mu_{1}+c_{2} \mu_{2}$, then $F(\mu)=F\left(\mu_{1}\right) \cap F\left(\mu_{2}\right)$. In other words, for any $\mathbf{x} \in \mathcal{X}$, we have $\mathbf{x} \in F\left(\mu_{1}\right) \cap F\left(\mu_{2}\right)$ if and only if $\mathbf{x} \in F(\mu)$.

In the setting of preference aggregation, Young and Levenglick (1978) showed that the Kemeny rule is the only rule which is neutral, anonymous, Condorcet admissible, and satisfies reinforcement. The Kemeny rule is simply the median rule on the permutahedron. The second main result of this paper is an analog of the Young-Levenglick theorem for judgement aggregation.

A gain function $\phi:[-1,1] \longrightarrow{ }^{*} \mathbb{R}$ is regular if there exists some $r_{2}>r_{1}>r_{0}>0$, such that $\phi\left(r_{0}\right) /\left(\phi\left(r_{2}\right)-\phi\left(r_{1}\right)\right)$ is finite. For example: any real-valued gain function is regular. To be irregular, $\phi$ must either be infinitely large on $(0,1]$, or have infinitesimal slope on this domain, or both. For example, if $\infty$ represents some infinite hyperreal, then the functions $\phi(r)=\operatorname{sign}(r) \cdot(\infty+\log |r|)$ and $\phi(r)=\operatorname{sign}(r) \cdot(1+\log |r| / \infty)$ are irregular. A judgement aggregation rule $F: \Delta(\mathcal{X}) \rightrightarrows \mathcal{X}$ is regular if $F=F_{\phi}$ for some regular $\phi$. We now come to our second main result.

Theorem 4.1 (a) The median rule is upper hemicontinuous and satisfies reinforcement on every judgement space.

(b) Let $\mathcal{X}$ be a thick judgement space, and let $F: \Delta(\mathcal{X}) \rightrightarrows \mathcal{X}$ be any judgement aggregation rule. Then $F$ is regular, upper hemicontinuous and satisfies reinforcement on $\Delta(\mathcal{X})$ if and only if $F$ is the median rule. ${ }^{3}$

Theorem 4.1 make it desirable to have an efficient way to compute the median rule. Of course, any additive rule can be computed by solving a linear program on the polyhedron

\footnotetext{
${ }^{3}$ Nehring and Pivato (2011b) give another reinforcement characterization of the median rule, which does not require $\mathcal{X}$ to be thick or require $F$ to be UHC, but instead imposes a slightly stronger regularity condition on the gain function near 0 .
} 
$\operatorname{conv}(\mathcal{X})$, but this may be quite time-consuming. However, for the median rule, there is another, possibly faster method, by traversing the internal graph $(\overline{\overline{\mathcal{X}}})$ from section 2 .

Let $\mu \in \Delta(\mathcal{X})$. Define an orientation $\underset{\mu}{\sim}$ on the internal graph by setting $\mathbf{x}_{\mu} \mathbf{y}$ if $\mathbf{x} \bullet \widetilde{\mu}<\mathbf{y} \bullet \widetilde{\mu}$, for any $\mathbf{x} \underset{\overline{\mathcal{X}}}{\mathbf{y}} \in \mathcal{X}$. This is an acyclic binary relation. Let $\underset{\mu}{\prec}$ be the transitive closure of $\underset{\mu}{\sim}$; then $\underset{\mu}{\prec}$ is a strict partial order on $\mathcal{X}$. Let $\max (\mathcal{X}, \underset{\mu}{\prec})$ be the set of $(\underset{\mu}{\prec})$-undominated elements in $\mathcal{X}$.

Proposition 4.2 For any judgement aggregation $\operatorname{problem}(\mathcal{X}, \mu)$, we have Median $(\mathcal{X}, \mu)=$ $\max (\mathcal{X}, \underset{\mu}{\prec})$.

\section{Neutral reinforcement and homogeneous rules}

Let $\mu^{1}, \mu^{2} \in \Delta(\mathcal{X})$, and let $c_{1}, c_{2} \in[0,1]$ with $c_{1}+c_{2}=1$. If $\mathbf{x} \in F\left(\mu^{1}\right)$ and $\mathbf{x} \in F\left(\mu^{2}\right)$, then the reinforcement axiom requires $\mathbf{x} \in F\left(c_{1} \mu^{1}+c_{2} \mu^{2}\right)$. One objection to this requirement is that the profiles $\mu^{1}$ and $\mu^{2}$ may choose $\mathbf{x}$ over other elements of $\mathcal{X}$ for two very different and incompatible reasons, and neither reason may be applicable for the combined profile $c_{1} \mu^{1}+c_{2} \mu^{2}$. For example, suppose $\mathbf{x} \in F\left(\mu^{1}\right)$ because there is some $k \in \mathcal{K}$ such that $x_{k} \widetilde{\mu}_{k}^{1} \approx$ 1 , while $\mathbf{x} \in F\left(\mu^{2}\right)$ because there is some other $k^{\prime} \in \mathcal{K} \backslash\{k\}$ such that $x_{k^{\prime}} \widetilde{\mu}_{k^{\prime}}^{2} \approx 1$. But perhaps there is no large supermajority supporting $\mathbf{x}$ in the combined profile $c_{1} \mu^{1}+c_{2} \mu^{2}$; thus, $\mathbf{x}$ might not be the best choice for $F\left(c_{1} \mu^{1}+c_{2} \mu^{2}\right)$. Reinforcement excludes this sort of argument; in this way, it excludes rules like leximin, which are strongly oriented towards satisfying large supermajorities. We will now consider a weakened form of reinforcement, which more tightly controls the mixing population $\mu^{2}$.

For any $\mathbf{x}, \mathbf{y} \in \mathcal{X}$, let $\delta^{\mathbf{x}, \mathbf{y}}:=\frac{1}{2} \delta_{\mathbf{x}}+\frac{1}{2} \delta_{\mathbf{y}}$. A judgement aggregation rule $F: \Delta(\mathcal{X}) \rightrightarrows \mathcal{X}$ satisfies neutral reinforcement if, for any $\mathbf{x}, \mathbf{y} \in \mathcal{X}$ and $\mu \in \Delta(\mathcal{X})$, if $F(\mu)=\{\mathbf{x}, \mathbf{y}\}$, then $F\left(r \mu+(1-r) \delta^{\mathbf{x}, \mathbf{y}}\right)=\{\mathbf{x}, \mathbf{y}\}$ for all $r \in(0,1]$. Reinforcement implies neutral reinforcement (because $\{\mathbf{x}, \mathbf{y}\} \subseteq F\left(\delta^{\mathbf{x}, \mathbf{y}}\right)$ for any supermajoritarian efficient rule $\left.F\right)$, but the latter condition is much weaker. For any $d \in(0, \infty)$, the $d$-homogeneous rule $H^{d}: \Delta(\mathcal{X}) \rightrightarrows \mathcal{X}$ is the additive support rule defined by $\phi^{d}(r):=\operatorname{sign}(r) \cdot|r|^{d}$.

Theorem $5.1 \quad$ (a) Any homogeneous rule is upper hemicontinuous and satisfies neutral reinforcement on every judgement space.

(b) Let $\mathcal{X}$ be a thick judgement space, and let $F: \Delta(\mathcal{X}) \rightrightarrows \mathcal{X}$ be a judgement aggregation rule. Then $F$ is regular, upper hemicontinuous and satisfies neutral reinforcement on $\Delta(\mathcal{X})$ if and only if $F=H^{d}$ for some $d \in(0, \infty){ }^{4}$

Homogeneous rules also satisfy two other, slightly stronger forms of neutral reinforcement.

Proposition 5.2 Let $F: \Delta(\mathcal{X}) \rightrightarrows \mathcal{X}$ be a homogeneous rule, let $\mathbf{x}, \mathbf{y} \in \mathcal{X}$, and let $\mu \in \Delta(\mathcal{X})$ such that $\mathbf{x} \in F(\mu)$. Then:

\footnotetext{
${ }^{4}$ Nehring and Pivato (2011b) give another neutral reinforcement characterization of homogeneous rules, which does not require $\mathcal{X}$ to be thick or $F$ to be UHC, but instead imposes a stronger regularity condition on the gain function near 0 .
} 
(a) $\mathbf{x} \in F\left(r \mu+(1-r) \delta^{\mathbf{x}, \mathbf{y}}\right)$ for all $r \in[0,1]$.

(b) Suppose there exists $\nu \in \Delta(\mathcal{X})$ such that $\widetilde{\nu}=\mathbf{0}$ (i.e. $\mathbf{0} \in \operatorname{conv}(\mathcal{X})$ ). Then $\mathbf{x} \in F(r \mu+(1-r) \nu)$ for all $r \in[0,1]$.

The proofs of both (a) and (b) are very similar to the proof of Theorem 5.1(a). Conversely, these properties also characterize homogeneous rules, via statements analogous to Theorem 5.1(b). However, these characterizations are less interesting, because the condition of Proposition 5.2(a) logically implies neutral reinforcement (so it yields a weaker characterization), and the condition of Proposition 5.2(b) only applies when $\mathbf{0} \in \operatorname{conv}(\mathcal{X})$.

\section{Continuity and uniqueness}

In $\S 3$ we showed that many SME rules can be represented as additive support rules. In what sense is the gain function $\phi$ in this representation unique? When is $\phi$ real-valued and continuous? In particular, Proposition 3.6 states that a real continuous gain function yields an upper hemicontinuous rule. Is the converse true?

The answer to these questions depends upon the structure of the judgement space $\mathcal{X}$. To see this, suppose that $\mathcal{X}$ is supermajoritarian determinate, in the sense defined in $\S 2$. Then for any gain functions $\phi$ and $\psi$, we have $F_{\phi}(\mathcal{X}, \mu)=F_{\psi}(\mathcal{X}, \mu)$ for all $\mu \in \Delta(\mathcal{X})$. In particular, the median rule and the leximin rule are identical on $\Delta(\mathcal{X})$. Thus, the additive representation is far from unique, and continuity of $\phi$ is not necessary for the upper hemicontinuity of $F_{\phi}$. This suggests that, in general, the additive representation of an SME rule on $\Delta(\mathcal{X})$ will be unique only to the extent that $\mathcal{X}$ deviates from supermajoritarian determinacy. Furthermore, we shall see that the uniqueness, real value, and continuity of the gain function $\phi$ can only be established in a subset $\mathcal{R}_{\mathcal{X}}^{\phi} \subseteq[-1,1]$, the 'domain of robust tradeoffs', which we now define.

Recall $\mathcal{C}:=\operatorname{conv}(\mathcal{X})$. Let $\mathcal{A}$ be the affine subspace of $\mathbb{R}^{\mathcal{K}}$ spanned by $\mathcal{C}$, and let int $(\mathcal{C})$ denote the relative interior of $\mathcal{C}$ as a subset of $\mathcal{A}$ (if $\mathcal{X}$ is thick, then this is just the interior of $\mathcal{C}$ as a subset of $\left.\mathbb{R}^{\mathcal{K}}\right)$. Let $\phi:[-1,1] \longrightarrow * \mathbb{R}$ be an odd, nondecreasing function. For any $\mathbf{x}, \mathbf{y} \in \mathcal{X}$, define

$$
{ }^{o} \mathcal{B}_{\mathbf{x}, \mathbf{y}}^{\phi}:=\left\{\mathbf{c} \in \operatorname{int}(\mathcal{C}) ; F_{\phi}(\mathbf{c})=\{\mathbf{x}, \mathbf{y}\}\right\} \quad \text { (this set may be empty). }
$$

For all $k \in \mathcal{K}_{ \pm}(\mathbf{x}, \mathbf{y})$, let $\mathcal{R}_{\mathbf{x}, \mathbf{y}}^{k}$ be the projection of ${ }^{\circ} \mathcal{B}_{\mathbf{x}, \mathbf{y}}^{F}$ onto the $k$ th coordinate. Define

$$
\mathcal{R}_{\mathcal{X}}^{\phi}:=\bigcup_{\substack{\mathbf{x}, \mathbf{y} \in \mathcal{X} \\ d(\mathbf{x}, \mathbf{y}) \geq 3}} \bigcup_{k \in \mathcal{K}_{ \pm}(\mathbf{x}, \mathbf{y})} \mathcal{R}_{\mathbf{x}, \mathbf{y}}^{k}
$$

Lemma 6.1 Let $\mathcal{X}$ be a judgement space which is not supermajoritarian determinate. If $\phi$ is any gain function such that $F_{\phi}$ is upper hemicontinuous on $\Delta(\mathcal{X})$, then $\mathcal{R}_{\mathcal{X}}^{\phi}$ is a nonempty open set.

In particular, Lemma 6.1 implies that $\mathcal{R}_{\mathcal{X}}^{\phi} \neq \emptyset$ if $\mathcal{X}$ thick and non-proximal. We now come to a partial converse to Proposition 3.6. 
Theorem 6.2 Let $\mathcal{X}$ be a thick judgement space, and let $\phi:[-1,1] \longrightarrow{ }^{*} \mathbb{R}$ be a gain function such that $F_{\phi}: \Delta(\mathcal{X}) \rightrightarrows \mathcal{X}$ is upper hemicontinuous and $\mathcal{R}_{\mathcal{X}}^{\phi} \neq \emptyset$.

(a) Let $\mathcal{R} \subseteq \mathcal{R}_{\mathcal{X}}^{\phi}$ be a connected component of $\mathcal{R}_{\mathcal{X}}^{\phi}$, and fix $r_{1}, r_{2} \in \mathcal{R}$ with $0<r_{1}<r_{2}$. Define $\bar{\phi}: \mathcal{R} \longrightarrow \mathbb{R}$ by

$$
\bar{\phi}(r):=\operatorname{st}\left(\frac{\phi(r)-\phi\left(r_{1}\right)}{\phi\left(r_{2}\right)-\phi\left(r_{1}\right)}\right),
$$

for all $r \in \mathcal{R}$. Then $\bar{\phi}$ is continuous, real-valued, and increasing on $\mathcal{R}$.

(b) Suppose there exists some $s \in{ }^{*} \mathbb{R}$ such that the function $\mathrm{st}(s \phi)$ is continuous and real-valued on $\mathrm{cl}\left(\mathcal{R}_{\mathcal{X}}^{\phi}\right)$. Then there exists a real-valued, continuous gain function $\psi:[-1,1] \longrightarrow \mathbb{R}$ such that $F_{\phi}=F_{\psi}$.

The next result shows that, even on a thick, non-proximal space, the 'full' converse to Proposition 3.6 is false: upper hemicontinuity of $F_{\phi}$ does not imply that $\phi$ is either continuous or real-valued everywhere on $[-1,1]$.

Proposition 6.3 Let $M \in \mathbb{N}$, and let $\mathcal{X}_{\mathcal{A}}^{\mathrm{pr}}$ be the permutahedron on $M$ alternatives. Let $\phi:[-1,1] \longrightarrow{ }^{*}$ be a gain function such that $\phi$ is continuous, real-valued, and unbounded on $\left(-1+\frac{2}{M}, 1-\frac{2}{M}\right)$, and $\phi$ is infinite on $\left[-1,-1+\frac{2}{M}\right] \sqcup\left[1-\frac{2}{M}, 1\right]$. Then $F_{\phi}$ is upper hemicontinuous.

Finally, we turn to the uniqueness of the additive representation. As with our results about continuity, it is only possible to establish uniqueness inside the domain $\mathcal{R}_{\mathcal{X}}^{\phi}$.

Theorem 6.4 Let $\phi:[-1,1] \longrightarrow \mathbb{R}$ and $\psi:[-1,1] \longrightarrow \mathbb{R}$ be odd, continuous and increasing. Let $\mathcal{X}$ be a thick judgement space, such that $\mathcal{R}_{\mathcal{X}}^{\phi} \cup\{0\}$ is connected.

We have $F_{\phi}(\mathcal{X}, \mu)=F_{\psi}(\mathcal{X}, \mu)$ for all $\mu \in \Delta(\mathcal{X})$ if and only if there is some $s>0$ such that $\psi(r)=s \phi(r)$ for all $r \in \mathcal{R}_{\mathcal{X}}^{\phi}$.

Thus, the behaviour of $F_{\phi}$ on $\Delta(\mathcal{X})$ uniquely determines the gain function $\phi$ (up to positive scalar multiplication) inside the region $\mathcal{R}_{\mathcal{X}}^{\phi}$. However, outside of $\mathcal{R}_{\mathcal{X}}^{\phi}$, the gain function $\phi$ can be redefined arbitrarily, without changing the behaviour of $F_{\phi}$.

When is $\mathcal{R}_{\mathcal{X}}^{\phi} \cup\{0\}$ is connected? A subset $\mathcal{S} \subset \mathbb{R}^{\mathcal{K}}$ is star-shaped (at $\mathbf{0}$ ) if, for any nonzero $\mathbf{s} \in \mathcal{S}$, the line segment $\operatorname{conv}\{\mathbf{0}, \mathbf{s}\}$ is contained in $\mathcal{S}$. For example, any convex set containing $\mathbf{0}$ is star-shaped. Let $\mathcal{C}:=\operatorname{conv}(\mathcal{X})$. For any $\phi:[-1,1] \longrightarrow \mathbb{R}$ and $\mathbf{c} \in \mathcal{C}$, recall that $\phi(\mathbf{c}):=\phi\left(c_{k}\right)_{k \in \mathcal{K}} \in \mathbb{R}^{\mathcal{K}}$. Then define $\phi[\mathcal{C}]:=\{\phi(\mathbf{c}) ; \mathbf{c} \in \mathcal{C}\}$, a subset of $\mathbb{R}^{\mathcal{K}}$.

Proposition 6.5 (a) If $\phi[\mathcal{C}]$ is star-shaped, then $\mathcal{R}_{\mathcal{X}}^{\phi} \cup\{0\}$ is connected.

(b) If $\mathbf{0} \in \mathcal{C}$, then for any $d \in(0, \infty)$, there exists $\epsilon>0$ such that, if $\left\|\phi-\phi^{d}\right\|_{\infty}<\epsilon$, then $\mathcal{R}_{\mathcal{X}}^{\phi} \cup\{0\}$ is connected.

(c) If $F_{\phi}: \Delta(\mathcal{X}) \rightrightarrows \mathcal{X}$ satisfies neutral reinforcement, then $\mathcal{R}_{\mathcal{X}}^{\phi} \cup\{0\}$ is connected.

Thus, Theorem 6.4 is applicable whenever $\phi$ is 'close enough' to some homogeneous rule. 


\section{Further directions}

Up until now, we have assumed that all voters have logically consistent judgements, so that the 'profile' could be represented as a function $\mu: \mathcal{X} \longrightarrow \mathbb{R}$. Furthermore, while different voters may have different weights, we assumed that a given voter should have the same weight with respect to all propositions in $\mathcal{K}$.

These assumptions are not always appropriate. Certain voters may have a special 'expertise' or 'priority' on certain propositions, but not on others. Indeed, perhaps they shouldn't have any weight at all on certain propositions. In a complex judgement aggregation problem, some voters might be 'specialists', whose judgement is reliable on a certain subset of $\mathcal{K}$, but whose overall judgement is not necessarily even an element of $\mathcal{X}$.

Fortunately, these assumptions are not necessary for our results. Suppose there are $N$ voters, and for each $k \in \mathcal{K}$, introduce a distinct weight function $\omega_{k}:[1 \ldots N] \longrightarrow \mathbb{R}^{+}$ (summing to unity), describing the relative priority of different voters on proposition $k$. Let $\left\{\mathbf{x}^{1}, \ldots, \mathbf{x}^{N}\right\} \in\{ \pm 1\}^{\mathcal{K}}$ be the judgements of the voters. (Thus, some voters could provide logically inconsistent judgements.) This information can no longer be be adequately summarized with a function $\mu:\{ \pm 1\}^{\mathcal{K}} \longrightarrow \mathbb{R}$. However, we can still define the vector $\widetilde{\mu} \in[-1,1]^{\mathcal{K}}$ by setting $\widetilde{\mu}_{k}:=\sum_{n=1}^{N} \omega_{k}(n) x_{k}^{n}$ for all $k \in \mathcal{K}$.

A judgement aggregation rule must now be defined as a multifunction $\widetilde{F}:[-1,1]^{\mathcal{K}} \rightrightarrows \mathcal{X}$. The information in $\widetilde{\mu}_{k}$ is all that is required to define supermajoritarian efficiency using the functions $\gamma_{\widetilde{\mu}, \mathbf{x}}$ defined in eqn.(4), or to define the additive rule $F_{\phi}$ via eqn.(6). Most of our results should generalize easily to this setting.

We have also assumed that all propositions in $\mathcal{K}$ should be treated as having equal importance. But in some judgement aggregation problems, certain propositions should receive special treatment. (For example, in truth-functional aggregation, 'premise' propositions should be treated differently from 'conclusion' propositions.)

To relax this assumption, we could introduce a weight vector $\boldsymbol{\lambda} \in \mathbb{R}_{+}^{\mathcal{K}}$, where $\lambda_{k}$ measures the 'importance' of coordinate $k$. For any $\mu \in \Delta(\mathcal{X}), \mathbf{x} \in \mathcal{X}$, and $q \in(0,1]$, we then define $\gamma_{\mu, \mathbf{x}}(q):=\sum\left\{\lambda_{k} ; k \in \mathcal{K}\right.$ and $\left.x_{k} \widetilde{\mu}_{k} \geq q\right\}$. We can then define supermajoritarian efficiency as before. For any $\phi:[-1,1] \longrightarrow \mathbb{L}$, we define

$$
F_{\phi}(\mathcal{X}, \lambda, \mu):=\underset{\mathbf{x} \in \mathcal{X}}{\operatorname{argmax}}\left(\sum_{k \in \mathcal{K}} \lambda_{k} \phi\left(x_{k} \widetilde{\mu}_{k}\right)\right) .
$$

Again, most of our results should generalize easily to this setting.

\section{Appendix A: Hyperreal fields}

Let $\mathcal{I}$ be any infinite indexing set, and let $\mathbb{R}^{\mathcal{I}}$ be the space of all functions $r: \mathcal{I} \longrightarrow \mathbb{R}$. A free filter on $\mathcal{I}$ is a collection $\mathfrak{F}$ of subsets of $\mathcal{I}$ satisfying the following axioms:

(F0) No finite subset of $\mathcal{I}$ is an element of $\mathfrak{F}$. (In particular, $\emptyset \notin \mathfrak{F}$.)

(F1) If $\mathcal{E}, \mathcal{F} \in \mathfrak{F}$, then $\mathcal{E} \cap \mathcal{F} \in \mathfrak{F}$. 
For any $\mathcal{E} \subseteq \mathcal{I}$, axioms $(\mathrm{F} 0)$ and $(\mathrm{F} 1)$ together imply that at most one of $\mathcal{E}$ or $\mathcal{E}^{\complement}$ can be in $\mathfrak{F}$. A free ultrafilter is filter $\mathfrak{F}$ which also satisfies:

(UF) For any $\mathcal{E} \subseteq \mathcal{I}$, either $\mathcal{E} \in \mathfrak{F}$ or $\mathcal{E}^{\complement} \in \mathfrak{F}$.

Equivalently, $\mathfrak{F}$ a 'maximal' filter: it is not a proper subset of any other filter. Heuristically, elements of $\mathfrak{F}$ are 'large' subsets of $\mathcal{I}$ : if $\mathcal{F} \in \mathfrak{F}$ and a certain statement holds for all $i \in \mathcal{F}$, then this statement holds for 'generic' element of $\mathcal{I}$. In particular, axioms (F0) and (UF) imply that $\mathcal{I} \in \mathfrak{F}$.

Ultrafilter lemma. Every free filter $\mathfrak{F}$ is contained in some free ultrafilter

Proof sketch. Consider the set of all free filters containing $\mathfrak{F}$; apply Zorn's Lemma to get a maximal element of this set.

Let $\mathfrak{F}$ be a free ultrafilter on $\mathcal{I}$, and for all $r, s \in \mathbb{R}^{\mathcal{I}}$, define $r \underset{\widetilde{F}}{\sim} s$ if the set $\{i \in \mathcal{I}$; $r(i)=s(i)\}$ is an element of $\mathfrak{F}$. Let ${ }^{*} \mathbb{R}:=\mathbb{R}^{\mathcal{I}} /(\underset{\widetilde{F}}{ })$. Then ${ }^{*} \mathbb{R}$ is both a linearly ordered field and a linearly ordered real vector space; it is called a hyperreal field. In particular, if $\mathcal{I}=\mathbb{N}$, then ${ }^{*} \mathbb{R}$ is the field of hyperreal numbers (Anderson, 1991).

\section{Appendix B: The internal graph}

Let $\mathcal{X}$ be a judgement space, and let $\mathcal{C}:=\operatorname{conv}(\mathcal{X})$. For any $\mathbf{x}, \mathbf{y} \in \mathcal{X}$, write "x $\overleftrightarrow{\mathcal{X}} \mathbf{y}$ " if some edge of the polyhedron $\mathcal{C}$ connects $\mathbf{x}$ to $\mathbf{y}$; this yields the edge graph of $\mathcal{X}$. This section will investigate the relationship between the internal graph and the edge graph. We will also consider a third graph structure on $\mathcal{X}$. For any $\mathbf{x}, \mathbf{y} \in \mathcal{X}$, define $\mathcal{K}_{0}(\mathbf{x}, \mathbf{y}):=\{k \in \mathcal{K}$; $\left.x_{k}=x_{y}\right\}$. Define $[\mathbf{x}, \mathbf{y}]:=\left\{\mathbf{z} \in \mathcal{X} ; z_{k}=x_{k}=y_{k}\right.$ for all $\left.k \in \mathcal{K}_{0}(\mathbf{x}, \mathbf{y})\right\}$. We write " $\mathbf{x}{ }_{\mathcal{X}}^{\bowtie} \mathbf{y}$ " if $[\mathbf{x}, \mathbf{y}]:=\{\mathbf{x}, \mathbf{y}\}$; this is the adjacency graph of $\mathcal{X}$.

For any $\mathbf{x}, \mathbf{y}, \mathbf{z} \in \mathcal{X}$, let $\operatorname{med}(\mathbf{x}, \mathbf{y}, \mathbf{z}) \in\{ \pm 1\}^{\mathcal{K}}$ be the outcome of a propositionwise majority vote amongst three voters who respectively endorse positions $\mathbf{x}, \mathbf{y}$, and $\mathbf{z}$. (Formally: $\operatorname{med}(\mathbf{x}, \mathbf{y}, \mathbf{z}):=\left(m_{k}\right)_{k \in \mathcal{K}}$, where for all $k \in \mathcal{K}, m_{k}:=\operatorname{sign}\left(x_{k}+y_{k}+z_{k}\right)$.) We say $\mathcal{X}$ is a median space if $\operatorname{med}(\mathbf{x}, \mathbf{y}, \mathbf{z}) \in \mathcal{X}$ for all $\mathbf{x}, \mathbf{y}, \mathbf{z} \in \mathcal{X}$. Nehring and Puppe (2007) have shown that $\mathcal{X}$ is a median space if and only if propositionwise majority vote on profiles in $\Delta(\mathcal{X})$ always produces outcomes in $\mathcal{X}$ (i.e. majority vote is a viable judgement aggregation rule); in this case all supermajoritarian efficient aggregation rules will agree with the outcome of majority vote. At the opposite extreme, we say $\mathcal{X}$ is McGarvey if $\mathbf{0} \in \operatorname{int}(\operatorname{conv}(\mathcal{X}))$. Nehring and Pivato (2011a) have shown that $\mathcal{X}$ is a McGarvey space if and only if propositionwise majority vote on profiles in $\Delta(\mathcal{X})$ can produce any outcome in $\{ \pm 1\}^{\mathcal{K}}$ (so any conceivable 'voting paradox' is possible).

Proposition B.1 (a) $\underset{\chi}{\ltimes}$ is a subgraph of $\overline{\overline{\bar{\chi}}}$.

(b) If $\mathcal{X}$ is a median space, then $\stackrel{\ltimes}{\mathcal{X}}$ is equal to $\overline{\overline{\mathcal{X}}}$. 
(c) $\overline{\overline{\mathcal{X}}}$ is a subgraph of $\overleftrightarrow{\mathcal{X}}$

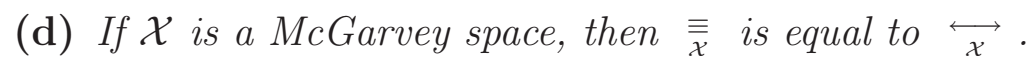

(e) The diameter of $\overline{\overline{\mathcal{X}}}$ is at most $\operatorname{dim}[\mathcal{C}]$.

Proof of Proposition B.1. Let $\mathbf{x}, \mathbf{y} \in \mathcal{X}$. Define $\mathcal{B}_{\mathbf{x}, \mathbf{y}}^{\prime}:=\{\mathbf{c} \in \mathcal{C} ; \operatorname{Median}(\mathbf{c})=\{\mathbf{x}, \mathbf{y}\}\}$. Recall that $D:=\operatorname{dim}[\mathcal{C}]$.

Claim 1: $\quad\left(\operatorname{dim}\left(\mathcal{B}_{\mathbf{x}, \mathbf{y}}\right)=D-1\right) \Longleftrightarrow\left(\mathcal{B}_{\mathbf{x}, \mathbf{y}}^{\prime} \neq \emptyset\right)$.

Proof: Let $\mathcal{P}:=\{\mathbf{c} \in \mathcal{C} ;(\mathbf{x}-\mathbf{y}) \bullet \mathbf{c}=0\} ;$ then $\mathcal{B}_{\mathbf{x}, \mathbf{y}}^{\prime} \subseteq \mathcal{B}_{\mathbf{x}, \mathbf{y}} \subset \mathcal{P}$. Clearly, $\operatorname{dim}(\mathcal{P})=D-1$. Thus, $\operatorname{dim}\left(\mathcal{B}_{\mathbf{x}, \mathbf{y}}\right)=D-1$ if and only if $\mathcal{B}_{\mathbf{x}, \mathbf{y}}$ contains a relatively open subset of $\mathcal{P}$.

“” For any $\mathbf{z} \in \mathcal{X} \backslash\{\mathbf{x}, \mathbf{y}\}$, let

$$
\mathcal{B}_{\mathbf{x}, \mathbf{y}, \mathbf{z}}:=\left\{\mathbf{c} \in \mathcal{B}_{\mathbf{x}, \mathbf{y}} ; \mathbf{z} \in F(\mathbf{c})\right\}=\left\{\mathbf{c} \in \mathcal{B}_{\mathbf{x}, \mathbf{y}} ;(\mathbf{x}-\mathbf{z}) \bullet \mathbf{c}=0\right\} .
$$

Then $\operatorname{dim}\left(\mathcal{B}_{\mathbf{x}, \mathbf{y}, \mathbf{z}}\right)=\operatorname{dim}\left(\mathcal{B}_{\mathbf{x}, \mathbf{y}}\right)-1=D-2$, because $\mathcal{B}_{\mathbf{x}, \mathbf{y}, \mathbf{z}}$ is defined by imposing an additional linear constraint on $\mathcal{B}_{\mathbf{x}, \mathbf{y}}$ (and $(\mathbf{x}-\mathbf{z})$ is linearly independent of $(\mathbf{x}-\mathbf{y})$ if $\mathbf{z} \neq \mathbf{y})$. Thus

$$
\mathcal{B}_{\mathbf{x}, \mathbf{y}}^{\prime}=\mathcal{B}_{\mathbf{x}, \mathbf{y}} \backslash \bigcup_{\mathbf{z} \in \mathcal{X} \backslash\{\mathbf{x}, \mathbf{y}\}} \mathcal{B}_{\mathbf{x}, \mathbf{y}, \mathbf{z}}
$$

is a $(D-1)$-dimensional set minus a finite union of lower-dimensional sets; hence it is nonempty.

"£" Suppose $\mathcal{B}_{\mathbf{x}, \mathbf{y}}^{\prime} \neq \emptyset$. Let $\mathbf{b} \in \mathcal{B}_{\mathbf{x}, \mathbf{y}}^{\prime}$. Then $\mathbf{b} \in \mathcal{P}$, and $(\mathbf{x}-\mathbf{z}) \bullet \mathbf{b}>0$, for all $\mathbf{z} \in \mathcal{X} \backslash\{\mathbf{x}, \mathbf{y}\}$. Let $\epsilon:=\min _{\mathbf{z} \in \mathcal{X} \backslash\{\mathbf{x}, \mathbf{y}\}}(\mathbf{x}-\mathbf{z}) \bullet \mathbf{b}$; then $\epsilon>0$, because $\mathcal{X}$ is finite.

For any $\mathbf{p} \in \mathbb{R}^{\mathcal{K}}$, if $\|\mathbf{b}-\mathbf{p}\|_{1}<\epsilon / 2$, then $(\mathbf{x}-\mathbf{z}) \bullet \mathbf{p}>0$ for all $\mathbf{z} \in \mathcal{X} \backslash\{\mathbf{x}, \mathbf{y}\}$ (because $\left.|(\mathbf{x}-\mathbf{z}) \bullet \mathbf{p}-(\mathbf{x}-\mathbf{z}) \bullet \mathbf{b}|<\|\mathbf{x}-\mathbf{z}\|_{\infty} \cdot\|\mathbf{p}-\mathbf{b}\|_{1}=2 \cdot\|\mathbf{p}-\mathbf{b}\|_{1}<2 \cdot \epsilon / 2=\epsilon\right)$. Thus, if $\mathbf{p} \in \mathcal{P}$ and $\|\mathbf{b}-\mathbf{p}\|_{1}<\epsilon / 2$, then $\mathbf{p} \in \mathcal{B}_{\mathbf{x}, \mathbf{y}}^{\prime}$. Thus, $\operatorname{dim}\left(\mathcal{B}_{\mathbf{x}, \mathbf{y}}^{\prime}\right)=\operatorname{dim}(\mathcal{P})=\operatorname{dim}(\mathcal{C})-1=$ $D-1$. But $\mathcal{B}_{\mathbf{x}, \mathbf{y}}^{\prime} \subseteq \mathcal{B}_{\mathbf{x}, \mathbf{y}} ;$ thus, $\operatorname{dim}\left(\mathcal{B}_{\mathbf{x}, \mathbf{y}}\right)=D-1 . \quad \diamond_{\text {claim } 1}$

(a) Suppose $\mathbf{x} \underset{\mathcal{X}}{\bowtie} \mathbf{y}$. We must show that $\mathbf{x} \underset{\bar{\chi}}{\equiv} \mathbf{y}$. Without loss of generality, suppose $x_{k}=1=$ $y_{k}$ for all $k \in \mathcal{K}_{0}(\mathbf{x}, \mathbf{y})$. Define $\mathbf{c}:=(\mathbf{x}+\mathbf{y}) / 2$. Then $c_{k}=1$ for all $k \in \mathcal{K}_{0}(\mathbf{x}, \mathbf{y})$, and $c_{k}=0$ for all other $k \in \mathcal{K}$. Thus, $\mathbf{c} \bullet \mathbf{x}=\mathbf{c} \bullet \mathbf{y}=\left|\mathcal{K}_{0}(\mathbf{x}, \mathbf{y})\right|$. For any other $\mathbf{z} \in \mathcal{X}$, we have $\mathbf{c} \bullet \mathbf{z} \leq\left|\mathcal{K}_{0}(\mathbf{x}, \mathbf{y})\right|$, with equality if and only if $\mathbf{z} \in[\mathbf{x}, \mathbf{y}]$. But $\mathbf{x}_{\mathcal{X}}^{\stackrel{凶}{y}}$, so $[\mathbf{x}, \mathbf{y}]=\{\mathbf{x}, \mathbf{y}\}$. Thus, $\mathbf{c} \bullet \mathbf{z}<\mathbf{c} \bullet \mathbf{x}=\mathbf{c} \bullet \mathbf{y}$ for all $\mathbf{z} \in \mathcal{X} \backslash\{\mathbf{x}, \mathbf{y}\}$. Thus, $F(\mathbf{c})=\{\mathbf{x}, \mathbf{y}\}$, so $\mathbf{c} \in \mathcal{B}_{\mathbf{x}, \mathbf{y}}^{\prime}$. Thus, $\mathcal{B}_{\mathbf{x}, \mathbf{y}}^{\prime} \neq \emptyset$, so Claim 1 says $\operatorname{dim}\left(\mathcal{B}_{\mathbf{x}, \mathbf{y}}\right)=D-1$ as desired.

(b) Suppose $\mathbf{x} / x_{x} \mathbf{y}$; we must show that $\mathbf{x} / F_{x} \mathbf{y}$. If $\left.\mathbf{x}\right|_{x} ^{x} \mathbf{y}$, then there exists some $\mathbf{z} \in$ $[\mathbf{x}, \mathbf{y}] \backslash\{\mathbf{x}, \mathbf{y}\}$; we will show that $\mathcal{B}_{\mathbf{x}, \mathbf{y}}=\mathcal{B}_{\mathbf{x}, \mathbf{y}, \mathbf{z}}$; thus, $\mathcal{B}_{\mathbf{x}, \mathbf{y}}^{\prime}=\emptyset$, and thus, Claim 1 implies that $\mathbf{x}$ 寿 $\mathbf{y}$.

Let $\widetilde{\mu} \in \mathcal{B}_{\mathbf{x}, \mathbf{y}}$; then $\{\mathbf{x}, \mathbf{y}\} \subseteq \operatorname{Median}(\mathcal{X}, \mu)$. But $\mathcal{X}$ is a median space, so $\operatorname{Median}(\mathcal{X}, \mu)=$ $\operatorname{Maj}(\mu)$. Thus, $\{\mathbf{x}, \mathbf{y}\} \subseteq \operatorname{Maj}(\mu)$. This means that $\operatorname{sign}\left(\widetilde{\mu}_{k}\right)=x_{k}=y_{k}$ for all $k \in$ $\mathcal{K}_{0}(\mathbf{x}, \mathbf{y})$, whereas $\operatorname{sign}\left(\widetilde{\mu}_{k}\right)=0$ for all $k \in \mathcal{K}_{ \pm}(\mathbf{x}, \mathbf{y})$. But $\mathbf{z} \in[\mathbf{x}, \mathbf{y}]$, so $z_{k}=x_{k}=y_{k}$ for all $k \in \mathcal{K}_{0}(\mathbf{x}, \mathbf{y})$. Thus, $\mathbf{z} \in \operatorname{Maj}(\mu)$ also. Thus, $\mathbf{z} \in \operatorname{Median}(\mathcal{X}, \mu)$. Thus, $\widetilde{\mu} \in \mathcal{B}_{\mathbf{x}, \mathbf{y}, \mathbf{z}}$, as claimed. 
(c) Suppose $\mathbf{x} \underset{\overline{\mathcal{X}}}{\equiv} \mathbf{y}$; we must show that $\mathbf{x} \underset{\mathcal{X}}{\overleftrightarrow{y}} \mathbf{y}$. Let $\mathbf{b} \in \mathcal{B}_{\mathbf{x}, \mathbf{y}}^{\prime}$. Then $\underset{\mathbf{z} \in \mathcal{X}}{\operatorname{argmax}}(\mathbf{b} \bullet \mathbf{z})=\{\mathbf{x}, \mathbf{y}\}$. Thus, $\operatorname{argmax}(\mathbf{b} \bullet \mathbf{c})=\operatorname{conv}\{\mathbf{x}, \mathbf{y}\}$ (because $\mathcal{X}$ contains all extremal points of $\mathcal{C}$ ). Thus, $\mathbf{c} \in \mathcal{C}$ $\operatorname{conv}\{\mathbf{x}, \mathbf{y}\}$ is the set of solutions to some linear program, so it is an edge of $\operatorname{conv}(\mathcal{X})$. Thus, $\mathbf{x} \underset{x}{\longleftrightarrow} \mathbf{y}$.

(d) Suppose $\mathbf{x} \underset{\mathcal{x}}{\longleftrightarrow} \mathbf{y}$; we must show that $\mathbf{x} \underset{\overline{\mathcal{X}}}{\overline{\mathbf{y}}} \mathbf{\text { . Since }} \operatorname{conv}\{\mathbf{x}, \mathbf{y}\}$ is an edge of $\mathcal{C}$, there exists some vector $\mathbf{v} \in \mathbb{R}^{\mathcal{K}}$ such that $\underset{\mathbf{c} \in \mathcal{C}}{\operatorname{argmax}}(\mathbf{v} \bullet \mathbf{c})=\operatorname{conv}\{\mathbf{x}, \mathbf{y}\}$. Now, $\mathcal{X}$ is McGarvey, so $\mathcal{C}$ contains a neighbourhood of $\mathbf{0}$, so there exists some $r>0$ such that $r \cdot \mathbf{v} \in \mathcal{C}$. Find some $\mu \in \Delta(\mathcal{X})$ such that $\widetilde{\mu}=r \cdot \mathbf{v}$. Then $\underset{\mathbf{c} \in \mathcal{C}}{\operatorname{argmax}}(\widetilde{\mu} \bullet \mathbf{c})=\operatorname{conv}\{\mathbf{x}, \mathbf{y}\}$; thus, $\underset{\mathbf{z} \in \mathcal{X}}{\operatorname{argmax}}(\widetilde{\mu} \bullet \mathbf{z})=\{\mathbf{x}, \mathbf{y}\}$. Thus, $\operatorname{Median}(\mathcal{X}, \mu)=\{\mathbf{c} \in \mathcal{C}, \mathbf{y}\}$. Thus, $\widetilde{\mu} \in \mathcal{B}_{\mathbf{x}, \mathbf{y}}^{\prime}$, so $\mathcal{B}_{\mathbf{x}, \mathbf{y}}^{\prime} \neq \emptyset$, so $\operatorname{dim}\left(\mathcal{B}_{\mathbf{x}, \mathbf{y}}\right)=D-1$ by Claim 1 above.

(e) Part (a) implies that it suffices to show that the diameter of the adjacency graph is at $\operatorname{most} \operatorname{dim}(\mathcal{C})$. Let $\mathbf{x}, \mathbf{z} \in \mathcal{X}$. A monotone path from $\mathbf{x}$ to $\mathbf{z}$ is a sequence $\mathbf{x}=$

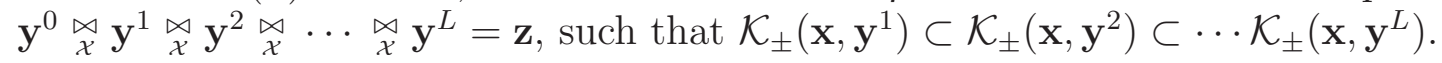

Claim 2: If $d(\mathbf{x}, \mathbf{z})=J$, then there is a monotone path from $\mathbf{x}$ to $\mathbf{z}$ of length at most $J$.

Proof: (by strong induction on $J$ )

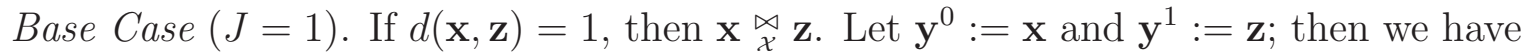
a monotone path of length 1 .

Induction. Suppose the claim is true for all $\mathbf{x}^{\prime}, \mathbf{z}^{\prime} \in \mathcal{X}$ with $d\left(\mathbf{x}^{\prime}, \mathbf{z}^{\prime}\right)<J$. Let

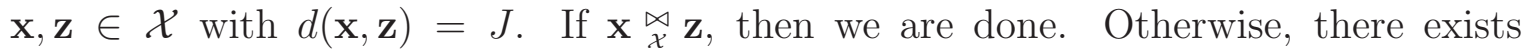
some $\mathbf{y} \in[\mathbf{x}, \mathbf{z}] \backslash\{\mathbf{x}, \mathbf{z}\}$. Thus, $x_{k}=y_{k}=z_{k}$ for all $k \in \mathcal{K}_{0}(\mathbf{x}, \mathbf{z})$. Furthermore, for all $k \in \mathcal{K}_{ \pm}(\mathbf{x}, \mathbf{z})$, either $y_{k}=x_{k}$, or $y_{k}=z_{k}$ (but not both). Thus, if $J^{\prime}:=d(\mathbf{x}, \mathbf{y})$ and $J^{\prime \prime}:=d(\mathbf{y}, \mathbf{z})$, then we have

$$
\begin{aligned}
J^{\prime}+J^{\prime \prime} & =\#\left\{k \in \mathcal{K}_{ \pm}(\mathbf{x}, \mathbf{z}) ; y_{k} \neq x_{k}\right\}+\#\left\{k \in \mathcal{K}_{ \pm}(\mathbf{x}, \mathbf{z}) ; y_{k} \neq z_{k}\right\} \\
& =\left|\mathcal{K}_{ \pm}(\mathbf{x}, \mathbf{z})\right|=d(\mathbf{x}, \mathbf{z})=J
\end{aligned}
$$

Also, $J^{\prime}, J^{\prime \prime} \geq 1$, because $\mathbf{x} \neq \mathbf{y} \neq \mathbf{z}$. Thus, $J^{\prime}=J-J^{\prime \prime} \leq J-1$ and $J^{\prime \prime}=J-J^{\prime} \leq J-1$. Thus, the induction hypothesis yields a monotone path from $\mathbf{x}$ to $\mathbf{y}$ of length at most $J^{\prime}$, and a monotone path from $\mathbf{y}$ to $\mathbf{z}$ of length at most $J^{\prime \prime}$. Gluing these two paths together yields a monotone path from $\mathbf{x}$ to $\mathbf{z}$ of length at most $J^{\prime}+J^{\prime \prime}=J . \quad \diamond$ claim 2

Now, let $\mathbf{x}, \mathbf{z} \in \mathcal{X}$; we must show that there is some $(\underset{\mathcal{X}}{\underset{\mathcal{X}}{\boldsymbol{x}}})$-path from $\mathbf{x}$ to $\mathbf{z}$ of length at

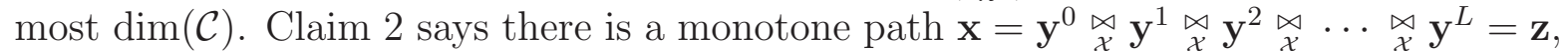
for some $L \in \mathbb{N}$. Since this path is monotone, the set $\mathcal{Y}:=\left\{\left(\mathbf{y}^{1}-\mathbf{y}^{0}\right),\left(\mathbf{y}^{2}-\mathbf{y}_{0}\right), \ldots,\left(\mathbf{y}^{L}-\right.\right.$ $\left.\left.\mathbf{y}^{0}\right)\right\}$ is linearly independent, which means that $\operatorname{dim}(\operatorname{span}(\mathcal{Y}))=L$. But $\mathcal{Y} \subseteq \mathcal{X}-\mathcal{X}$, so $\operatorname{dim}(\operatorname{span}(\mathcal{Y})) \leq \operatorname{dim}(\operatorname{span}(\mathcal{X}-\mathcal{X}))=\operatorname{dim}(\mathcal{C})$. Thus, we must have $L \leq \operatorname{dim}(\mathcal{C})$, as desired. 


\section{Appendix C: Detecting identical SME aggregation rules}

Throughout this section, let $\mathcal{X} \subset\{ \pm 1\}^{\mathcal{K}}$ be some judgement space. This section contains technical results which are useful for showing that two judgement aggregation rules $F$ and $G$ are actually identical. For example, the proofs of Theorems 2.1 and 3.4(c) both use the following result.

Lemma C.1 Let $F, G: \Delta(\mathcal{X}) \rightrightarrows \mathcal{X}$ be two judgement aggregation rules. Suppose $F(\mu) \subseteq$ $G(\mu)$ for all $\mu \in \Delta(\mathcal{X})$, and $G$ is monotone, and $F$ is upper hemicontinuous. Then $F(\mu)=G(\mu)$ for all $\mu \in \Delta(\mathcal{X})$.

Proof: Let $\mu \in \Delta(\mathcal{X})$. We have $F(\mu) \subseteq G(\mu)$ by hypothesis; we must show $F(\mu) \supseteq G(\mu)$. So, let $\mathbf{x} \in G(\mu)$; we will show that $\mathbf{x} \in F(\mu)$. Let $\delta_{\mathbf{x}} \in \Delta(\mathcal{X})$ be the point mass at $\mathbf{x}$. For all $n \in \mathbb{N}$, define $\mu_{n}:=\left(1-\frac{1}{n}\right) \mu+\frac{1}{n} \delta_{\mathbf{x}}$. Then $\mu_{n}$ is more supportive of $\mathbf{x}$ than $\mu$, so $G\left(\mu_{n}\right)=\{\mathbf{x}\}$ because $G$ is monotone. Thus, $F\left(\mu_{n}\right)=\{\mathbf{x}\}$ because $F \subseteq G$, and $F\left(\mu_{n}\right)$ must be nonempty. However, $\lim _{n \rightarrow \infty} \mu_{n}=\mu$, and $F$ is upper hemicontinuous. Thus, $\mathbf{x} \in F(\mu)$, as desired.

A judgement aggregation rule $F: \Delta(\mathcal{X}) \rightrightarrows \mathcal{X}$ is a support rule if there exists a function $\widetilde{F}: \operatorname{conv}(\mathcal{X}) \rightrightarrows \mathcal{X}$ such that $F(\mu)=\widetilde{F}(\widetilde{\mu})$ for all $\mu \in \Delta(\mathcal{X})$. For example: for any odd gain function $\phi:[-1,1] \longrightarrow{ }^{*} \mathbb{R}$, the additive rule $F_{\phi}$ is a support rule, because Lemma 3.2(b) says that $F_{\phi}(\mu)$ is simply the set of $\mathbf{x} \in \mathcal{X}$ which maximize the value of $\mathbf{x} \bullet \phi(\widetilde{\mu})$.

Let $\mathcal{C}:=\operatorname{conv}(\mathcal{X})$, and let $F: \mathcal{C} \rightrightarrows \mathcal{X}$ be a support rule. For any $\mathrm{x} \in \mathcal{X}$, we define

$$
\mathcal{C}_{\mathbf{x}}^{F}:=\{\mathbf{c} \in \mathcal{C} ; \mathbf{x} \in F(\mathbf{c})\} \quad \text { and } \quad{ }^{\circ} \mathcal{C}_{\mathbf{x}}^{F} \quad:=\quad\{\mathbf{c} \in \mathcal{C} ; F(\mathbf{c})=\{\mathbf{x}\}\} .
$$

Next, for any $\mathbf{x}, \mathbf{y} \in \mathcal{X}$, we define

$$
\mathcal{B}_{\mathbf{x}, \mathbf{y}}^{F}:=\mathcal{C}_{\mathbf{x}}^{F} \cap \mathcal{C}_{\mathbf{y}}^{F}=\{\mathbf{c} \in \mathcal{C} ; \mathbf{x}, \mathbf{y} \in F(\mathbf{c})\} .
$$

A key step in the proof of Theorem E.7 is the following result:

Proposition C.2 Let $F, G: \Delta(\mathcal{X}) \rightrightarrows \mathcal{X}$ be SME, UHC monotone support rules. Then

$$
(F=G) \Longleftrightarrow\left({ }^{o} \mathcal{B}_{\mathbf{x}, \mathbf{y}}^{F} \subseteq \mathcal{B}_{\mathbf{x}, \mathbf{y}}^{G} \cup\left(\mathcal{C} \backslash \mathcal{C}_{\mathbf{x}}^{G}\right) \text { for every } \mathbf{x}, \mathbf{y} \in \mathcal{X} \text { with } d(\mathbf{x}, \mathbf{y}) \geq 3\right) \text {. }
$$

In fact, Proposition C.2 follows from a more general result, which is also used to prove Theorem 5.1(b).

Proposition C.3 Let $F, G: \Delta(\mathcal{X}) \rightrightarrows \mathcal{X}$ be UHC, monotone support rules. Then

$$
(F=G) \Longleftrightarrow\left({ }^{o} \mathcal{B}_{\mathbf{x}, \mathbf{y}}^{F} \subseteq \mathcal{B}_{\mathbf{x}, \mathbf{y}}^{G} \cup\left(\mathcal{C} \backslash \mathcal{C}_{\mathbf{x}}^{G}\right) \text { for every } \mathbf{x}, \mathbf{y} \in \mathcal{X}\right)
$$


Remark. Note that, for any $\mathbf{x}, \mathbf{y} \in \mathcal{X}$ and any rules $F, G: \Delta(\mathcal{X}) \rightrightarrows \mathcal{X}$, we have

$$
\left({ }^{o} \mathcal{B}_{\mathbf{x}, \mathbf{y}}^{F} \subseteq \mathcal{B}_{\mathbf{x}, \mathbf{y}}^{G} \cup\left(\mathcal{C} \backslash \mathcal{C}_{\mathbf{x}}^{G}\right)\right) \quad \Longleftrightarrow \quad\left({ }^{\circ} \mathcal{B}_{\mathbf{x}, \mathbf{y}}^{F} \cap \mathcal{C}_{\mathbf{x}}^{G} \subseteq \mathcal{C}_{\mathbf{y}}^{G}\right)
$$

Thus, we can use the right hand side of eqn.(C1) when applying Propositions C.2 and C.3.

We need two preliminary lemmas to prove Proposition C.3.

Lemma C.4 Let $F: \Delta(\mathcal{X}) \rightrightarrows \mathcal{X}$ be an upper hemicontinuous, monotone support rule. Let $\mathbf{x} \in \mathcal{X}$. Let $\partial \mathcal{C}_{\mathbf{x}}^{F}$ be the relative boundary of $\mathcal{C}_{\mathbf{x}}^{F}$ as a subset of $\mathcal{C}$, and let int $\left(\mathcal{C}_{\mathbf{x}}^{F}\right)$ be the relative interior of $\mathcal{C}_{\mathbf{x}}^{F}$. (That is: $\partial \mathcal{C}_{\mathbf{x}}^{F}:=\mathcal{C}_{\mathbf{x}}^{F} \cap \operatorname{cl}\left(\mathcal{C} \backslash \mathcal{C}_{\mathbf{x}}^{F}\right)$ and $\operatorname{int}\left(\mathcal{C}_{\mathbf{x}}^{F}\right):=\mathcal{C}_{\mathbf{x}}^{F} \backslash \partial \mathcal{C}_{\mathbf{x}}^{F}$.) Then:

(a) $\operatorname{int}\left(\mathcal{C}_{\mathbf{x}}^{F}\right)={ }^{o} \mathcal{C}_{\mathbf{x}}^{F}$

(b) $\mathcal{C}_{\mathbf{x}}^{F}$ is connected.

(c) $\mathcal{C}_{\mathrm{x}}^{F}=\operatorname{cl}\left({ }^{\circ} \mathcal{C}_{\mathrm{x}}^{F}\right)$.

(d) $\partial \mathcal{C}_{\mathbf{x}}^{F}=\bigcup_{\mathbf{y} \in \mathcal{X} \backslash\{\mathbf{x}\}} \mathcal{B}_{\mathbf{x}, \mathbf{y}}^{F}=\bigcup_{\mathbf{y} \in \mathcal{X} \backslash\{\mathbf{x}\}} \operatorname{cl}\left({ }^{o} \mathcal{B}_{\mathbf{x}, \mathbf{y}}^{F}\right)$.

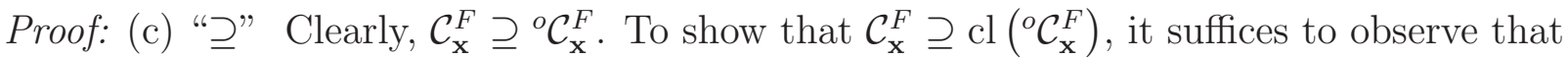
$\mathcal{C}_{\mathbf{x}}^{F}$ is closed, because $F$ is upper hemicontinuous by hypothesis.

" $\subseteq$ " Let $\mathbf{c} \in \mathcal{C}_{\mathbf{x}}^{F}$. For any $r \in(0,1)$, let $\mathbf{c}^{r}:=r \mathbf{x}+(1-r) \mathbf{c}$. Clearly, $\lim _{r \rightarrow 0} \mathbf{c}^{r}=\mathbf{c}$. Thus, to show that $\mathbf{c} \in \operatorname{cl}\left({ }^{o} \mathcal{C}_{\mathbf{x}}^{F}\right)$, it suffices to show that $\mathbf{c}^{r} \in{ }^{o} \mathcal{C}_{\mathbf{x}}^{F}$ for all $r>0$.

To see this, suppose $\mathbf{c}=\widetilde{\mu}$ for some $\mu \in \Delta(\mathcal{X})$. Define $\delta_{\mathbf{x}} \in \Delta(\mathcal{X})$ as in eqn.(9). Then $\mathbf{c}^{r}=\widetilde{\mu}^{r}$, where $\widetilde{\mu}^{r}=r \delta_{\mathbf{x}}+(1-r) \mu$. Now, $\widetilde{\mu}^{r}$ is more supportive of $\mathbf{x}$ than $\mu$; thus, $F(\widetilde{\mu})=\{\mathbf{x}\}$, because $F$ is monotone by hypothesis. Thus, $\widetilde{F}\left(\mathbf{c}^{r}\right)=\{\mathbf{x}\}$; hence $\mathbf{c}^{r} \in{ }^{o} \mathcal{C}_{\mathbf{x}}^{F}$.

(b) For any $\mathbf{c} \in{ }^{\circ} \mathcal{C}_{\mathbf{x}}^{F}$, the proof of part (c) shows that the line segment from $\mathbf{x}$ to $\mathbf{c}$ is in ${ }^{\circ} \mathcal{C}_{\mathbf{x}}^{F}$. Thus, ${ }^{\mathcal{C}}{ }_{\text {x }}^{F}$ is path-connected, hence connected.

(a) To see $\operatorname{int}\left(\mathcal{C}_{\mathbf{x}}^{F}\right) \supseteq{ }^{{ }^{\mathcal{C}}} F$, note that

$$
{ }^{o} \mathcal{C}_{\mathbf{x}}^{F}:=\quad\{\mathbf{c} \in \mathcal{C} ; F(\mathbf{c})=\mathbf{x}\}=\mathcal{C} \backslash \bigcup_{\mathbf{y} \in \mathcal{X} \backslash\{\mathbf{x}\}} \mathcal{C}_{\mathbf{y}}^{F} .
$$

Now $\bigcup_{\mathbf{y} \in \mathcal{X} \backslash\{\mathbf{x}\}} \mathcal{C}_{\mathbf{y}}^{F}$ is closed because $\mathcal{X}$ is finite and $\mathcal{C}_{\mathbf{y}}^{F}$ is closed for any $\mathbf{y} \in \mathcal{X}$ (because $F$ is upper hemicontinuous). Thus, eqn.(C2) makes ${ }^{{ }^{\circ}} \mathcal{C}_{\mathbf{x}}^{F}$ a relatively open subset of $\mathcal{C}$; thus, ${ }^{\mathcal{C}_{\mathrm{x}}^{F}} \subseteq \operatorname{int}\left(\mathcal{C}_{\mathrm{x}}^{F}\right)$

To see $\operatorname{int}\left(\mathcal{C}_{\mathrm{x}}^{F}\right) \subseteq{ }^{{ }^{F}} F$, note that

$$
\begin{aligned}
\operatorname{cl}\left(\mathcal{C} \backslash{ }^{\circ} \mathcal{C}_{\mathbf{x}}^{F}\right) & \overline{\overline{(*)}} \bigcup_{\mathbf{y} \in \mathcal{X} \backslash\{\mathbf{x}\}} \mathcal{C}_{\mathbf{y}}^{F} \overline{\overline{(\diamond)}} \bigcup_{\mathbf{y} \in \mathcal{X} \backslash\{\mathbf{x}\}} \operatorname{cl}\left({ }^{\circ} \mathcal{C}_{\mathbf{y}}^{F}\right) \\
& \overline{\overline{(\dagger)}} \operatorname{cl}\left(\bigcup_{\mathbf{y} \in \mathcal{X} \backslash\{\mathbf{x}\}}{ }^{o} \mathcal{C}_{\mathbf{y}}^{F}\right) \subseteq \operatorname{cl}\left(\mathcal{C} \backslash \mathcal{C}_{\mathbf{x}}^{F}\right) .
\end{aligned}
$$


Here, $(*)$ is by eqn. $(\mathrm{C} 2),(\diamond)$ is by applying part (c) to each $\mathbf{y} \in \mathcal{X} \backslash\{\mathbf{x}\}$, and $(\dagger)$ is because $\mathcal{X}$ is finite.

Taking the complement of both sides, we get $\mathcal{C} \backslash \operatorname{cl}\left(\mathcal{C} \backslash \mathcal{C}_{\mathrm{x}}^{F}\right) \supseteq \mathcal{C} \backslash \operatorname{cl}\left(\mathcal{C} \backslash \mathcal{C}_{\mathrm{x}}^{F}\right)$, which is equivalent to $\operatorname{int}\left({ }^{\circ} \mathcal{C}_{\mathbf{x}}^{F}\right) \supseteq \operatorname{int}\left(\mathcal{C}_{\mathbf{x}}^{F}\right)$, which means ${ }^{~} \mathcal{C}_{\mathbf{x}}^{F} \supseteq \operatorname{int}\left(\mathcal{C}_{\mathbf{x}}^{F}\right)$ (because ${ }^{~} \mathcal{C}_{\mathbf{x}}^{F}$ is relatively open).

(d) We must first establish some results about the geometry of $\mathcal{C}_{\mathbf{x}}^{F}$.

Claim 1: For any $\mathbf{b} \in \operatorname{int}(\mathcal{C}) \cap \partial \mathcal{C}_{\mathbf{x}}^{F}$, there exist arbitrarily small relative neighbourhoods $\mathcal{V} \subseteq \mathcal{C}$ around $\mathbf{b}$ such that the set $\mathcal{V} \backslash \mathcal{C}_{\mathbf{x}}^{F}$ is path-connected.

Proof: Monotonicity implies that $\mathbf{x} \in \mathcal{C}_{\mathbf{x}}^{F}$. Thus, upper hemicontinuity implies that there is some $\epsilon>0$ such that $\mathcal{B}(\mathbf{x}, \epsilon) \cap \mathcal{C} \subseteq{ }^{\mathcal{C}_{\mathbf{x}}^{F}}$ (where $\mathcal{B}(\mathbf{x}, \epsilon)$ is the $\epsilon$-ball around $\mathbf{x}$ in $\left.\mathbb{R}^{\mathcal{K}}\right)$. Let $\mathcal{S}:=\partial \mathcal{B}(\mathbf{x}, \epsilon) \cap \mathcal{C}$; then $\mathcal{S}$ is homeomorphic to a ball in $\mathbb{R}^{D-1}$. Thus, the Cartesian product $\mathcal{S} \times[0,1]$ is homeomorphic to a $D$-dimensional 'cylinder'.

Let $\mathcal{C}^{*}:=\mathcal{C} \backslash\{\mathbf{x}\}$ and let $\mathcal{C}_{\mathbf{x}}^{*}:=\mathcal{C}_{\mathbf{x}}^{F} \backslash\{\mathbf{x}\}$.

Claim 1.1: (a) There is a homeomorphism $\eta: \mathcal{C}^{*} \longrightarrow \mathcal{S} \times(0,1]$.

(b) There is a continuous function $\alpha: \mathcal{S} \longrightarrow(0,1]$ such that $\eta\left(\mathcal{C}_{\mathbf{x}}^{*}\right)=\{(s, t) ; s \in \mathcal{S}$ and $0<t \leq \alpha(s)\}$.

Proof: (a) For any $\mathbf{c} \in \mathcal{C}^{*}$, let $\mathcal{L}_{\mathbf{c}}$ denote the unique ray in $\mathbb{R}^{\mathcal{K}}$ originating at $\mathbf{x}$ and passing through c. Then $\mathcal{L}_{\mathbf{c}}$ passes through $\mathcal{S}$ at a unique point - call this point $\sigma(\mathbf{c})$. This defines a continuous surjection $\sigma: \mathcal{C}^{*} \longrightarrow \mathcal{S}$. Let $\mathcal{T}$ be the set of all faces of the polyhedron $\mathcal{C}$ which do not contain $\mathbf{x}$. Then for any $\mathbf{c} \in \mathcal{C}^{*}$, the line $\mathcal{L}_{\mathbf{c}}$ passes through $\mathcal{T}$ at a unique point - call this point $\tau(\mathbf{c})$. This defines a continuous function $\tau: \mathcal{C}^{*} \longrightarrow \mathcal{T}$. Let $\rho(\mathbf{c}):=|\mathbf{c}-\mathbf{x}| /|\tau(\mathbf{c})-\mathbf{x}|$; this defines a continuous function $\rho: \mathcal{C}^{*} \longrightarrow(0,1]$ (with $\left.\mathcal{T}=\rho^{-1}\{1\}\right)$. Furthermore, for any fixed $\mathbf{s} \in \mathcal{S}$, the restricted map $\rho: \mathcal{L}_{\mathbf{s}} \cap \mathcal{C}^{*} \longrightarrow(0,1]$ is bijective. Now define $\eta: \mathcal{C}^{*} \longrightarrow \mathcal{S} \times(0,1]$ by $\eta(\mathbf{c}):=(\sigma(\mathbf{c}), \rho(\mathbf{c}))$; then $\eta$ is a homeomorphism.

(b) For any $\mathbf{c} \in \mathcal{C}^{*}$, let $(\mathbf{x}, \mathbf{c}]$ denote the line segment from $\mathbf{x}$ to $\mathbf{c}$ (a subset of $\mathcal{L}_{\mathbf{c}}$ ). If $\mathbf{c} \in \mathcal{C}_{\mathbf{x}}^{F}$, then $(\mathbf{x}, \mathbf{c}] \subset \mathcal{C}_{\mathbf{x}}^{F}$ (because $F$ is monotone). If $\eta(\mathbf{c})=\left(\mathbf{s}, r_{0}\right)$, then this means that $\eta^{-1}(\mathbf{s}, r) \in \mathcal{C}_{\mathbf{x}}^{F}$ for all $r \in\left(0, r_{0}\right]$. Thus, for any $\mathbf{s} \in \mathcal{S}$, if we define

$$
\alpha(\mathbf{s}):=\sup \left\{\rho(\mathbf{c}) ; \mathbf{c} \in \mathcal{C}_{\mathbf{x}}^{F} \cap \mathcal{L}_{\mathbf{s}}\right\}=\sup \left\{r \in[0,1] ; \eta^{-1}(\mathbf{s}, r) \in \mathcal{C}_{\mathbf{x}}^{F}\right\},
$$

then $\eta^{-1}(\mathbf{s}, r) \in \mathcal{C}_{\mathbf{x}}^{F}$ for all $r<\alpha(\mathbf{s})$. Thus, $\eta^{-1}(\mathbf{s}, \alpha(\mathbf{s})) \in \mathcal{C}_{\mathbf{x}}^{F}$, because $\mathcal{C}_{\mathbf{x}}^{F}$ is closed because $F$ is upper hemicontinuous. Furthermore, $\eta^{-1}(\mathbf{s}, \alpha(\mathbf{s})) \in \partial \mathcal{C}_{\mathbf{x}}^{F}$ if $\alpha(\mathbf{s})<1$ (again by upper hemicontinuity).

The set $\mathcal{S}$ is compact, and $\rho: \mathcal{S} \longrightarrow(0,1]$ is continuous; thus, $M:=\min \{\rho(\mathbf{s}) ; \mathbf{s} \in \mathcal{S}\}$ exists, and $M>0$. Clearly, $\alpha(\mathbf{s}) \geq M$ for all $\mathbf{s} \in \mathcal{S}$ (because $\mathcal{S} \subset \mathcal{C}_{\mathbf{x}}^{F}$ ).

To see that $\alpha$ is continuous, let $\left\{\mathbf{s}_{n}\right\}_{n=1}^{\infty} \subset \mathcal{S}$ be a sequence converging to some point $\mathbf{s} \in \mathcal{S}$, and let $t_{n}:=\alpha\left(\mathbf{s}_{n}\right)$ for all $n \in \mathbb{N}$. Then $\left\{t_{n}\right\}_{n=1}^{\infty} \subset[M, 1]$, by the previous paragraph. The interval $[M, 1]$ is compact, so the sequence $\left\{t_{n}\right\}_{n=1}^{\infty}$ must have cluster points in $[M, 1]$. Let $t$ be any cluster point of $\left\{t_{n}\right\}_{n=1}^{\infty}$; we must show that $\alpha(\mathbf{s})=t$. First suppose $t<1$. By dropping to a subsequence if necessary, we can ensure that $t_{n}<1$ for all large enough $n \in \mathbb{N}$; thus $\eta^{-1}\left(s, t_{n}\right) \in \partial \mathcal{C}_{\mathbf{x}}^{F}$. The map $\eta^{-1}$ is 
continuous; thus, $\eta^{-1}(\mathbf{s}, t)$ is a cluster point of the sequence $\left\{\eta^{-1}\left(\mathbf{s}_{n}, t_{n}\right)\right\}_{n=1}^{\infty} \subset \partial \mathcal{C}_{\mathbf{x}}^{F}$; thus, $\eta^{-1}(\mathbf{s}, t) \in \partial \mathcal{C}_{\mathbf{x}}^{F}$ (because $\partial \mathcal{C}_{\mathbf{x}}^{F}$ is closed, by upper hemicontinuity). Thus, $\alpha(\mathbf{s})=t$ (by definition of $\alpha$ ).

Thus, if $\left\{t_{n}\right\}_{n=1}^{\infty} \subset \mathcal{S}$ clusters at any point $t<1$, then $\alpha(\mathbf{s})=t$. Otherwise, we must have $\lim _{n \rightarrow \infty} t_{n}=1$. But $\left\{\eta^{-1}\left(\mathbf{s}_{n}, t_{n}\right)\right\}_{n=1}^{\infty} \subset \mathcal{C}_{\mathbf{x}}^{F}$; thus, $\eta^{-1}(\mathbf{s}, 1) \in \mathcal{C}_{\mathbf{x}}^{F}$ (because $\mathcal{C}_{\mathbf{x}}^{F}$ is closed, by upper hemicontinuity). Thus, $\alpha(\mathbf{s})=1$.

$\nabla$ Claim 1.1

Now, let $\mathbf{b} \in \operatorname{int}(\mathcal{C}) \cap \partial \mathcal{C}_{\mathbf{x}}^{F}$, and let $\left(\mathbf{s}_{0}, t_{0}\right):=\eta(\mathbf{b})$. Then $t_{0}<1$ (because $\mathbf{b} \notin \partial \mathcal{C}$ because $\mathbf{b} \in \operatorname{int}(\mathcal{C}))$. But $t_{0}=\alpha\left(\mathbf{s}_{0}\right)$; thus, $\alpha(\mathbf{s})<1$ for all $\mathbf{s}$ sufficiently close to $\mathbf{s}_{0}$ (because $\alpha$ is continuous, by Claim 1.1(b)). Furthermore, for any $\epsilon>0$, there exists some $\delta>0$ such that the set

$$
\left\{(\mathbf{s}, t) \in \mathcal{S} \times(0,1] ;\left|\mathbf{s}-\mathbf{s}_{0}\right|<\delta \text { and } \alpha(\mathbf{s})<t<\alpha\left(\mathbf{s}_{0}\right)+\epsilon\right\}
$$

is path-connected (because $\alpha$ is continuous). Thus, there exist arbitrarily small neighbourhoods $\mathcal{U}$ around $\left(\mathbf{s}_{0}, t_{0}\right)$ such that the set $\{(\mathbf{s}, t) \in \mathcal{U} ; \alpha(\mathbf{s})<t<1\}$ is path connected. Mapping this back through the homeomorphism $\eta$ from Claim 1.1(a), this means there exist arbitrarily small neighbourhoods $\mathcal{V}$ around $\mathbf{b}$ such that the set $\mathcal{V} \backslash \mathcal{C}_{\mathbf{x}}^{F}$ is path-connected.

$\diamond$ Claim 1

For any $\mathcal{Y} \subseteq \mathcal{X}$, define ${ }^{\circ} \mathcal{B}_{\mathcal{Y}}^{F}:=\{\mathbf{c} \in \mathcal{C} ; F(\mathbf{c})=\mathcal{Y}\}$. (Thus, if $\mathcal{Y}=\{\mathbf{x}, \mathbf{y}\}$, then ${ }^{\circ} \mathcal{B}_{\mathbf{x}, \mathbf{y}}^{F}=$ ${ }^{\circ} \mathcal{B}_{\mathcal{Y}}^{F} \cap \operatorname{int}(\mathcal{C})$.) If $\mathbf{y} \in \mathcal{Y}$ and $|\mathcal{Y}| \geq 2$, then clearly ${ }^{\circ} \mathcal{B}_{\mathcal{Y}}^{F} \subset \partial \mathcal{C}_{\mathbf{y}}^{F}$.

Claim 2: Let $\mathbf{x}_{0} \in \mathcal{Y} \subseteq \mathcal{X}$ with $|\mathcal{Y}| \geq 3$. Then every element of ${ }^{\circ} \mathcal{B}_{\mathcal{Y}}^{F} \cap \operatorname{int}(\mathcal{C})$ is a cluster point of ${ }^{\circ} \mathcal{B}_{y^{\prime}}^{F}$ for some $\mathcal{Y}^{\prime} \subsetneq \mathcal{Y}$ with $\mathrm{x}_{0} \in \mathcal{Y}^{\prime}$ and $2 \leq\left|\mathcal{Y}^{\prime}\right| \leq|\mathcal{Y}|-1$.

Proof: Suppose $\mathcal{Y}:=\left\{\mathbf{x}_{0}, \mathbf{x}_{1}, \ldots, \mathbf{x}_{N}\right\}$ (for some $N \geq 2$ ). Let $\mathbf{b} \in{ }^{\circ} \mathcal{B}_{\mathcal{Y}}^{F} \cap \operatorname{int}(\mathcal{C})$. We will show that $\mathbf{b}$ is a cluster point of ${ }^{\circ} \mathcal{B}_{\mathcal{Y}^{\prime}}^{F}$ for some $\mathcal{Y}^{\prime} \subseteq\left\{\mathbf{x}_{0}, \mathbf{x}_{1}, \ldots, \mathbf{x}_{N-1}\right\}$ with $\left|\mathcal{Y}^{\prime}\right| \geq 2$ and $\mathbf{x}_{0} \in \mathcal{Y}^{\prime}$.

There exists some relatively open neighbourhood $\mathcal{V} \subset \mathcal{C}$ around $\mathbf{b}$ such that, for all $\mathbf{b}^{\prime} \in \mathcal{V}$, we have $F\left(\mathbf{b}^{\prime}\right) \subseteq \mathcal{Y}$ (otherwise, we contradict the upper hemicontinuity of $F$, because $F(\mathbf{b})=\mathcal{Y})$. Now, $\mathbf{b} \in\left(\partial \mathcal{C}_{\mathbf{x}_{N}}^{F}\right) \cap \operatorname{int}(\mathcal{C})$; thus Claim 1 says we can choose $\mathcal{V}$ such that the set $\mathcal{V} \backslash \mathcal{C}_{\mathbf{x}_{N}}^{F}$ is path-connected.

For any $\epsilon>0$, let $\mathbf{b}_{0}^{\epsilon}:=\epsilon \mathbf{x}_{0}+(1-\epsilon) \mathbf{b}$ and let $\mathbf{b}_{1}^{\epsilon}:=\epsilon \mathbf{x}_{1}+(1-\epsilon) \mathbf{b}$. Then $F\left(\mathbf{b}_{0}^{\epsilon}\right)=\left\{\mathbf{x}_{0}\right\}$ and $F\left(\mathbf{b}_{1}^{\epsilon}\right)=\left\{\mathbf{x}_{1}\right\}$ because $F$ is monotone. If $\epsilon$ is small enough, then $\mathbf{b}_{0}^{\epsilon}, \mathbf{b}_{1}^{\epsilon} \in \mathcal{V}$ (it's a neighbourhood of $\mathbf{b}$ ) and clearly $\mathbf{b}_{0}^{\epsilon}, \mathbf{b}_{1}^{\epsilon} \notin \mathcal{C}_{\mathbf{x}_{N}}^{F}$; thus $\mathbf{b}_{0}^{\epsilon}, \mathbf{b}_{1}^{\epsilon} \in \mathcal{V} \backslash \mathcal{C}_{\mathbf{x}_{N}}^{F}$, which is path-connected. So, let $\beta^{\mathcal{V}}:[0,1] \longrightarrow \mathcal{V} \backslash \mathcal{C}_{\mathbf{x}_{N}}^{F}$ be a continuous path with $\beta^{\mathcal{V}}(0)=$ $\mathbf{b}_{0}^{\epsilon}$ and $\beta^{\mathcal{V}}(1)=\mathbf{b}_{1}^{\epsilon}$. Thus, $F\left(\beta^{\mathcal{V}}(0)\right)=\left\{\mathbf{x}_{0}\right\}$ and $F\left(\beta^{\mathcal{V}}(1)\right)=\left\{\mathbf{x}_{1}\right\}$. Thus, upper hemicontinuity of $F$ yields some $R_{\mathcal{V}} \in[0,1)$ such that $\mathbf{x}_{0} \in F\left(\beta^{\mathcal{V}}(r)\right)$ for all $r \in\left[0, R_{\mathcal{V}}\right]$, but $\mathbf{x}_{0} \notin F\left(\beta^{\mathcal{V}}(r)\right)$ for all $r \in\left(R_{\mathcal{V}}, 1\right]$. But $F\left(\beta^{\mathcal{V}}(r)\right) \neq \emptyset$; thus, for all $r \in\left(R_{\mathcal{V}}, 1\right]$, we must have $\mathbf{x}_{n(r)} \in F\left(\beta^{\mathcal{V}}(r)\right)$ for some $n(r) \in[1 \ldots N-1]$. By letting $r \searrow R_{\mathcal{V}}$, invoking upper hemicontinuity, and dropping to a subsequence if necessary, we get $\mathbf{x}_{n} \in F\left(\beta^{\mathcal{V}}\left(R_{\mathcal{V}}\right)\right)$ for some $n \in[1 \ldots N-1]$. Thus, we have $\left\{\mathbf{x}_{0}, \mathbf{x}_{n}\right\} \subseteq F\left(\beta^{\mathcal{V}}\left(R_{\mathcal{V}}\right)\right) \subseteq$ $\left\{\mathbf{x}_{1}, \ldots, \mathbf{x}_{N-1}\right\}$. In other words, $F\left(\beta^{\mathcal{V}}\left(R_{\mathcal{V}}\right)\right)=\mathcal{Y}^{\prime}$ for some $\mathcal{Y}^{\prime} \subseteq\left\{\mathbf{x}_{0}, \ldots, \mathbf{x}_{N-1}\right\}$ with $\left|\mathcal{Y}^{\prime}\right| \geq 2$ and $\mathbf{x}_{0} \in \mathcal{Y}^{\prime}$.

Let $\left\{\mathcal{V}_{n}\right\}_{n=1}^{\infty}$ be a sequence of neighbourhoods of $\mathbf{b}$ satisfying the conditions of Claim 1 , whose diameters converge to 0 . For every $n \in \mathbb{N}$, construct $\mathbf{b}^{n}:=\beta^{\mathcal{V}_{n}}\left(R_{\mathcal{V}_{n}}\right)$ as 
in the previous paragraph. Then $\mathbf{b}^{n} \underset{\epsilon \rightarrow 0}{\longrightarrow} \mathbf{b}$, because $\mathbf{b}^{n} \in \mathcal{V}^{n}$, and $\operatorname{diam}\left(\mathcal{V}_{n}\right) \underset{n \rightarrow \infty}{\longrightarrow} 0$. By dropping to a subsequence if necessary, we can ensure there is some fixed subset $\mathcal{Y}^{\prime} \subseteq\left\{\mathbf{x}_{0}, \ldots, \mathbf{x}_{N-1}\right\}$ with $\left|\mathcal{Y}^{\prime}\right| \geq 2$ and $\mathbf{x}_{0} \in \mathcal{Y}^{\prime}$, such that $F\left(\mathbf{b}^{n}\right)=\mathcal{Y}^{\prime}$ for all $n \in \mathbb{N}$. Thus, $\mathbf{b}$ is a cluster point of ${ }^{\circ} \mathcal{B}_{\mathcal{Y}^{\prime}}^{F}$.

$\diamond$ Claim 2

Claim 3: Let $\mathrm{x} \in \mathcal{Y} \subset \mathcal{X}$ with $|\mathcal{Y}| \geq 2$. Then every element of ${ }^{\circ} \mathcal{B}_{\mathcal{Y}}^{F}$ is a cluster point of ${ }^{\circ} \mathcal{B}_{\mathbf{x}, \mathbf{y}}^{F}$ for some $\mathbf{y} \in \mathcal{Y} \backslash\{\mathbf{x}\}$.

Proof: Every element of ${ }^{\circ} \mathcal{B}_{\mathcal{Y}}^{F}$ is a cluster point of ${ }^{\circ} \mathcal{B}_{\mathcal{Y}}^{F} \cap \operatorname{int}(\mathcal{C})$. Thus, it suffices to prove the claim for elements of ${ }^{\circ} \mathcal{B}_{\mathcal{Y}}^{F} \cap \operatorname{int}(\mathcal{C})$.

Let $|\mathcal{Y}|=N \geq 2$. If $N=2$ then we are done. So suppose $N>2$. Let $\mathbf{b} \in{ }^{o} \mathcal{B}_{\mathcal{Y}}^{F} \cap \operatorname{int}(\mathcal{C})$. Claim 2 says that $\mathbf{b}=\lim _{n \rightarrow \infty} \mathbf{b}_{n}$ for some sequence $\left\{\mathbf{b}_{n}\right\}_{n=1}^{\infty} \subseteq{ }^{\circ} \mathcal{B}_{\mathcal{Y}^{\prime}}$, where $\mathcal{Y}^{\prime} \subsetneq \mathcal{Y}$ with $2 \leq\left|\mathcal{Y}^{\prime}\right| \leq|\mathcal{Y}|-1$ and $\mathrm{x} \in \mathcal{Y}^{\prime}$

If $\left|\mathcal{Y}^{\prime}\right|=2$, then we are done. Otherwise, for each $n \in \mathbb{N}$, Claim 2 implies that $\mathbf{b}_{n}=\lim _{m \rightarrow \infty} \mathbf{b}_{n, m}$ for some sequence $\left\{\mathbf{b}_{n, m}\right\}_{m=1}^{\infty} \subseteq{ }^{\circ} \mathcal{B}_{\mathcal{Y}_{n}}^{F}$, where $\mathcal{Y}_{n} \subsetneq \mathcal{Y}^{\prime}$ with $2 \leq\left|\mathcal{Y}_{n}\right| \leq$ $\left|\mathcal{Y}^{\prime}\right|-1$ and $\mathbf{x} \in \mathcal{Y}_{n}$. By dropping to a subsequence of $\left\{\mathbf{b}_{n}\right\}_{n=1}^{\infty}$ if necessary, we can assume that $\mathcal{Y}_{n}=\mathcal{Y}^{\prime \prime}$ for all $n \in \mathbb{N}$, for some fixed $\mathcal{Y}^{\prime \prime} \subseteq \mathcal{Y}^{\prime}$ with $2 \leq\left|\mathcal{Y}^{\prime \prime}\right| \leq\left|\mathcal{Y}^{\prime}\right|-1$ and $\mathbf{x} \in \mathcal{Y}^{\prime \prime}$. Now consider the diagonal sequence $\left\{\mathbf{b}_{n, n}\right\}_{n=1}^{\infty}$. Clearly, $\mathbf{b}_{n, n} \underset{n \rightarrow \infty}{\longrightarrow} \mathbf{b}$; thus, b is a cluster point of ${ }^{\circ} \mathcal{B}_{\mathcal{Y}^{\prime \prime}}^{F}$.

If $\left|\mathcal{Y}^{\prime \prime}\right|=2$, then we are done. Otherwise, repeat the argument of the previous paragraph to construct a sequence $\left\{\mathbf{b}_{n, n, n}\right\}_{n=1}^{\infty} \subseteq{ }^{o} \mathcal{B}_{\mathcal{Y}^{\prime \prime \prime}}^{F}$, for some $\mathcal{Y}^{\prime \prime \prime} \subsetneq \mathcal{Y}^{\prime \prime}$ with $2 \leq\left|\mathcal{Y}^{\prime \prime \prime}\right| \leq\left|\mathcal{Y}^{\prime \prime}\right|-1$ and $\mathbf{x} \in \mathcal{Y}^{\prime \prime \prime}$, such that $\mathbf{b}_{n, n, n} \underset{n \rightarrow \infty}{\longrightarrow} \mathbf{b}$.

Inductively, we can find a decreasing sequence $\mathcal{Y} \supsetneq \mathcal{Y}^{\prime} \supsetneq \mathcal{Y}^{\prime \prime} \supsetneq \mathcal{Y}^{\prime \prime \prime} \supsetneq \cdots \supsetneq \mathcal{Y}^{(k)} \supsetneq$ $\cdots\{\mathbf{x}\}$, such that $\mathbf{b}$ is a cluster point of ${ }^{\circ} \mathcal{B}_{\mathcal{Y}^{(k)}}^{F}$ and $\left|\mathcal{Y}^{(k)}\right| \geq 2$ for every $k$. Since $|\mathcal{Y}|$ is finite, we must eventually reach some $k$ where $\left|\mathcal{Y}^{(k)}\right|=2$ and $\mathbf{x} \in \mathcal{Y}^{(k)} . \quad \diamond$ claim 3

Now we have:

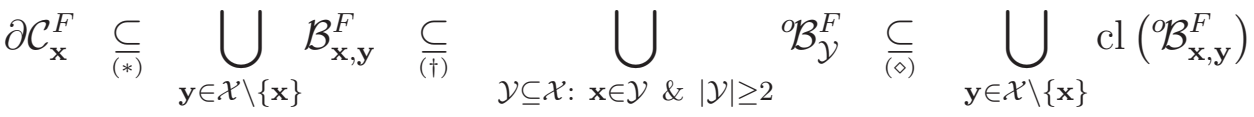

$$
\begin{aligned}
& \underset{( \pm)}{\subset} \bigcup_{\mathbf{y} \in \mathcal{X} \backslash\{\mathbf{x}\}} \mathcal{B}_{\mathbf{x}, \mathbf{y}}^{F} \underset{(@)}{\subseteq} \partial \mathcal{C}_{\mathbf{x}}^{F}
\end{aligned}
$$

To see $(*)$, let $\mathbf{b} \in \partial \mathcal{C}_{\mathbf{x}}^{F}$. Then $\mathbf{b}$ is a cluster point of $\mathcal{C}_{\mathbf{x}}^{G}$, but also a cluster point of $\mathcal{C}_{\mathbf{y}}^{F}$ for some $\mathbf{y} \in \mathcal{X} \backslash\{\mathbf{x}\}$. Then upper hemicontinuity of $F$ implies that $\mathbf{b} \in \mathcal{C}_{\mathbf{x}}^{F} \cap \mathcal{C}_{\mathbf{y}}^{F}=\mathcal{B}_{\mathbf{x}, \mathbf{y}}^{F}$. Next, $(\dagger)$ is because, for all $\mathbf{y} \in \mathcal{X} \backslash\{\mathbf{x}\}$, we have $\mathcal{B}_{\mathbf{x}, \mathbf{y}}^{F} \subseteq \mathcal{B}_{\mathcal{Y}}^{F}$ for some $\mathcal{Y} \supseteq\{\mathbf{x}, \mathbf{y}\}$. Meanwhile, $(\diamond)$ is by Claim 3. Finally, $(\ddagger)$ is because for all $\mathbf{x}, \mathbf{y} \in \mathcal{X}$, we have $\operatorname{cl}\left({ }^{\circ} \mathcal{B}_{\mathbf{x}, \mathbf{y}}^{F}\right) \subseteq$ $\mathcal{B}_{\mathbf{x}, \mathbf{y}}$ by upper hemicontinuity.

To see (@), let $\mathbf{y} \in \mathcal{X} \backslash\{\mathbf{x}\}$. For any $\mathbf{b} \in \mathcal{B}_{\mathbf{x}, \mathbf{y}}^{F}$, monotonicity of $F$ implies that $\mathbf{b}$ is a cluster point of both ${ }^{\circ} \mathcal{C}_{\mathbf{x}}^{F}$ and ${ }^{\circ} \mathcal{C}_{\mathbf{y}}^{F}$; thus, $\mathcal{B}_{\mathbf{x}, \mathbf{y}} \subseteq \partial \mathcal{C}_{\mathbf{x}}^{F}$.

Lemma C.5 Let $F, G: \Delta(\mathcal{X}) \rightrightarrows \mathbb{R}$ be upper hemicontinuous, monotone support rules. The following are equivalent: 
(a) $\mathcal{C}_{\mathrm{x}}^{F} \subseteq \mathcal{C}_{\mathrm{x}}^{G}$ for all $\mathrm{x} \in \mathcal{X}$.

(b) ${ }^{\circ} \mathcal{C}_{\mathrm{x}}^{F} \subseteq{ }^{o} \mathcal{C}_{\mathrm{x}}^{G}$ for all $\mathrm{x} \in \mathcal{X}$.

(c) $F=G$.

Proof: The statement "(c) $\Longrightarrow(\mathrm{b})$ " is immediate. The statement "(b) $\Longrightarrow$ (a)" follows Lemma C.4(c). It remains to show "(a) $\Longrightarrow(\mathrm{c})$ ". So suppose $\mathcal{C}_{\mathrm{x}}^{F} \subseteq \mathcal{C}_{\mathrm{x}}^{G}$ for all $\mathrm{x} \in \mathcal{X}$.

Claim 1: For all $\mathrm{x} \in \mathcal{X}$, we have: (i) ${ }^{\circ} \mathcal{C}_{\mathrm{x}}^{G} \subseteq{ }^{{ }^{C}} \mathcal{x}_{\mathbf{x}}^{F}$, and (ii) $\operatorname{cl}\left({ }^{\circ} \mathcal{C}_{\mathbf{x}}^{G}\right) \subseteq \operatorname{cl}\left({ }^{\circ} C_{\mathbf{x}}^{F}\right)$.

Proof: (i) Suppose $\mathbf{c} \in \mathcal{C}_{\mathbf{x}}^{G}$. Then for all $\mathbf{y} \in \mathcal{X} \backslash\{\mathbf{x}\}, \mathbf{c} \notin \mathcal{C}_{\mathbf{y}}^{G}$; hence the contrapositive of hypothesis (a) says $\mathbf{c} \notin \mathcal{C}_{\mathbf{y}}^{F}$, so $\mathbf{y} \notin F(\mathbf{c})$. Thus, if $\mathbf{x} \notin F(\mathbf{c})$, then $F(\mathbf{c})=\emptyset$, which is impossible. Thus, $\mathbf{x} \in F(\mathbf{c})$, which means that $F(\mathbf{c})=\{\mathbf{x}\}$; hence $\mathbf{c} \in \mathcal{C}^{\mathcal{F}}{ }_{\mathbf{x}}$.

(ii) Follows immediately by taking the closure in part (i).

$\diamond$ Claim 1

Claim 2: $\quad$ For all $\mathrm{x} \in \mathcal{X}$, we have $\mathcal{C}_{\mathrm{x}}^{G}=\mathcal{C}_{\mathrm{x}}^{F}$.

Proof: Combining Claim 1(ii) and Lemma C.4(c), we get $\mathcal{C}_{\mathrm{x}}^{G} \subseteq \mathcal{C}_{\mathrm{x}}^{F}$. But $\mathcal{C}_{\mathrm{x}}^{G} \supseteq \mathcal{C}_{\mathrm{x}}^{F}$ by hypothesis. Thus, $\mathcal{C}_{\mathrm{x}}^{G}=\mathcal{C}_{\mathrm{x}}^{F}$.

Thus, for any $\mathbf{c} \in \mathcal{C}$ and any $\mathbf{x} \in \mathcal{X}$, we have

$$
(\mathrm{x} \in F(\mathrm{c})) \Longleftrightarrow\left(\mathrm{c} \in \mathcal{C}_{\mathrm{x}}^{F}\right) \Longleftrightarrow\left(\mathrm{c} \in \mathcal{C}_{\mathrm{x}}^{G}\right) \Longleftrightarrow(\mathrm{x} \in G(\mathrm{c})),
$$

where $(*)$ is by Claim 2. Thus, $F=G$.

Proof of Proposition C.3. " $\Longrightarrow$ " is obvious: if $F=G$, then ${ }^{\circ} \mathcal{B}_{\mathbf{x}, \mathbf{y}}^{F}={ }^{\circ} \mathcal{B}_{\mathbf{x}, \mathbf{y}}^{G} \subseteq \mathcal{B}_{\mathbf{x}, \mathbf{y}}^{G}$ for all $\mathbf{x}, \mathbf{y} \in \mathcal{X}$.

"£" According to Lemma C.5, it suffices to establish the following statement:

$$
\mathcal{C}_{\mathrm{x}}^{G} \subseteq \mathcal{C}_{\mathrm{x}}^{F}, \quad \text { for every } \mathrm{x} \in \mathcal{X} .
$$

To verify statement (C3), first note that

$$
\begin{aligned}
& \partial \mathcal{C}_{\mathbf{x}}^{F} \overline{\overline{(*)}} \bigcup_{\mathbf{y} \in \mathcal{X} \backslash\{\mathbf{x}\}} \operatorname{cl}\left({ }^{\circ} \mathcal{B}_{\mathbf{x}, \mathbf{y}}^{F}\right) \underset{(\overline{(o)}}{\subset} \bigcup_{\mathbf{y} \in \mathcal{X} \backslash\{\mathbf{x}\}} \mathcal{B}_{\mathbf{x}, \mathbf{y}}^{G} \cup \operatorname{cl}\left(\mathcal{C} \backslash \mathcal{C}_{\mathbf{x}}^{G}\right) \\
& \overline{\overline{(\dagger)}} \partial \mathcal{C}_{\mathbf{x}}^{G} \cup \operatorname{cl}\left(\mathcal{C} \backslash \mathcal{C}_{\mathbf{x}}^{G}\right) \quad \overline{\overline{(\ddagger)}} \operatorname{cl}\left(\mathcal{C} \backslash \mathcal{C}_{\mathbf{x}}^{G}\right) .
\end{aligned}
$$

Here, $(*)$ is by applying Lemma C.4(d) to $\partial \mathcal{C}_{\mathbf{x}}^{F},(\diamond)$ is by the theorem hypothesis, and $(\dagger)$ is by applying Lemma C.4(d) to $\partial \mathcal{C}_{\mathbf{x}}^{G}$. Finally ( $\left.\ddagger\right)$ is because $\partial \mathcal{C}_{\mathrm{x}}^{G} \subseteq \operatorname{cl}\left(\mathcal{C} \backslash \mathcal{C}_{\mathrm{x}}^{G}\right)$ by definition.

It follows that

$$
\operatorname{int}\left(\mathcal{C}_{\mathbf{x}}^{F}\right) \sqcup \operatorname{int}\left(\mathcal{C} \backslash \mathcal{C}_{\mathbf{x}}^{F}\right) \quad=\mathcal{C} \backslash\left(\partial \mathcal{C}_{\mathbf{x}}^{F}\right) \underset{(*)}{\supset} \mathcal{C} \backslash \operatorname{cl}\left(\mathcal{C} \backslash \mathcal{C}_{\mathbf{x}}^{G}\right)=\operatorname{int}\left(\mathcal{C}_{\mathbf{x}}^{G}\right),
$$

where $(*)$ is by equation $(\mathrm{C} 4)$. But $\operatorname{int}\left(\mathcal{C}_{\mathrm{x}}^{G}\right)$ is connected, by Lemma C.4(b). Thus, we must have either int $\left(\mathcal{C}_{\mathrm{x}}^{G}\right) \subseteq \operatorname{int}\left(\mathcal{C}_{\mathrm{x}}^{F}\right)$, or int $\left(\mathcal{C}_{\mathrm{x}}^{G}\right) \subseteq \operatorname{int}\left(\mathcal{C} \backslash \mathcal{C}_{\mathrm{x}}^{F}\right)$. However, $\mathrm{x} \in \operatorname{int}\left(\mathcal{C}_{\mathrm{x}}^{G}\right)$ and $\mathbf{x} \in \operatorname{int}\left(\mathcal{C}_{\mathbf{x}}^{F}\right)$ (by monotonicity). Thus, we must have $\operatorname{int}\left(\mathcal{C}_{\mathbf{x}}^{G}\right) \subseteq \operatorname{int}\left(\mathcal{C}_{\mathbf{x}}^{F}\right)$. Take the closures and apply Lemma C.4(c) to get $\mathcal{C}_{\mathrm{x}}^{G} \subseteq \mathcal{C}_{\mathrm{x}}^{F}$, as desired. 
To go from Proposition C.3 to Proposition C.2, we need two more lemmas, which analyze the way SME rules distinguish between elements of $\mathcal{X}$ with Hamming distance 1 or 2. The first of these is also used in the proof of Proposition 3.6.

Lemma C.6 Let $\mu \in \Delta(\mathcal{X})$ and let $\mathbf{x}, \mathbf{y} \in \mathcal{X}$ with $d(\mathbf{x}, \mathbf{y}) \leq 2$.

(a) If $\mathbf{x} \bullet \widetilde{\mu}<\mathbf{y} \bullet \widetilde{\mu}$, then $\mathbf{x} \underset{\mu}{\triangleleft} \mathbf{y}$, and thus, $\mathbf{x} \notin \operatorname{SME}(\mathcal{X}, \mu)$.

(b) If $\mathbf{x} \bullet \widetilde{\mu}=\mathbf{y} \bullet \tilde{\mu}$, then $\gamma_{\mu, \mathbf{x}}=\gamma_{\mu, \mathbf{y}}$. Thus, $\mathbf{x} \in \operatorname{SME}(\mathcal{X}, \mu)$ if and only if $\mathbf{y} \in$ $\operatorname{SME}(\mathcal{X}, \mu)$

Proof: (a) Suppose $d(\mathbf{x}, \mathbf{y})=1$, and $\mathcal{K}_{ \pm}(\mathbf{x}, \mathbf{y})=\{j\}$. Then $(\mathbf{x}-\mathbf{y}) \bullet \widetilde{\mu}=\left(x_{j}-y_{j}\right) \widetilde{\mu}_{j}$. Suppose without loss of generality that $y_{j}=1$. Thus, $x_{j}=-1$ and

$$
\begin{aligned}
& (\mathbf{x} \bullet \tilde{\mu}<\mathbf{y} \bullet \tilde{\mu}) \Longleftrightarrow\left(\left(x_{j}-y_{j}\right) \widetilde{\mu}_{j}<0\right) \quad \Longrightarrow \quad\left(\widetilde{\mu}_{j}>0\right) \\
& \Longrightarrow \quad\left(\gamma_{\mathbf{x}, \mu}(r)=\left\{\begin{array}{rll}
\gamma_{\mathbf{y}, \mu}(r) & \text { if } & r>\widetilde{\mu} \\
\gamma_{\mathbf{y}, \mu}(r)-1 & \text { if } & r \leq \widetilde{\mu}
\end{array}\right)\right. \\
& \Longrightarrow\left(\mathbf{x}_{\mu}^{\triangleleft} \mathbf{y}\right) \Longrightarrow(\mathrm{x} \notin \operatorname{SME}(\mathcal{X}, \mu)) \text {. }
\end{aligned}
$$

To see $(\diamond)$, recall that $x_{k}=y_{k}$ for all $k \in \mathcal{K} \backslash\{j\}$.

Now suppose $d(\mathbf{x}, \mathbf{y})=2$, and $\mathcal{K}_{ \pm}(\mathbf{x}, \mathbf{y})=\{i, j\}$. Then $(\mathbf{x}-\mathbf{y}) \bullet \widetilde{\mu}=\left(x_{i}-y_{i}\right) \widetilde{\mu}_{i}+\left(x_{j}-\right.$ $\left.y_{j}\right) \tilde{\mu}_{j}$. Suppose without loss of generality that $0 \leq \widetilde{\mu}_{i} \leq \widetilde{\mu}_{j}$. Thus

$$
\begin{aligned}
(\mathbf{x} \bullet \tilde{\mu}<\mathbf{y} \bullet \tilde{\mu}) & \Longleftrightarrow\left(\left(x_{i}-y_{i}\right) \widetilde{\mu}_{i}+\left(x_{j}-y_{j}\right) \widetilde{\mu}_{j}<0\right) \\
& \Longleftrightarrow\left(\left(x_{i}-y_{i}\right) \widetilde{\mu}_{i}<\left(y_{j}-x_{j}\right) \widetilde{\mu}_{j}\right) \Longrightarrow \quad\left(y_{j}=1 \text { and } x_{j}=-1\right) .
\end{aligned}
$$

But $x_{k}=y_{k}$ for all $k \in \mathcal{K} \backslash\{i, j\}$. Thus:

- If $x_{i}=1$ and $y_{i}=-1$, then $\gamma_{\mu, \mathbf{x}}(r)=\left\{\begin{array}{rll}\gamma_{\mathbf{y}, \mu}(r) & \text { if } & r>\widetilde{\mu} ; \\ \gamma_{\mu, \mathbf{y}}(r)-1 & \text { if } & \widetilde{\mu}_{i}<r \leq \widetilde{\mu}_{j} ; \\ \gamma_{\mu, \mathbf{y}}(r) & \text { if } & r \leq \widetilde{\mu}_{i} .\end{array}\right.$
- If $x_{i}=-1$ and $y_{i}=1$, then $\gamma_{\mu, \mathbf{x}}(r)=\left\{\begin{array}{rll}\gamma_{\mathbf{y}, \mu}(r) & \text { if } & r>\widetilde{\mu} ; \\ \gamma_{\mu, \mathbf{y}}(r)-1 & \text { if } \quad \widetilde{\mu}_{i}<r \leq \widetilde{\mu}_{j} ; \\ \gamma_{\mu, \mathbf{y}}(r)-2 & \text { if } \quad r \leq \widetilde{\mu}_{i} .\end{array}\right.$

Either way, $\mathbf{x} \underset{\mu}{\triangleleft} \mathbf{y}$, and thus, $\mathbf{x} \notin \operatorname{SME}(\mathcal{X}, \mu)$.

(b) The proof is similar to (a). In the case $d(\mathbf{x}, \mathbf{y})=1$, we deduce that $\widetilde{\mu}_{j}=0$. In the case $d(\mathbf{x}, \mathbf{y})=2$, we deduce that $\widetilde{\mu}_{j}=\widetilde{\mu}_{j}$. Either way, we then deduce that $\gamma_{\mu, \mathbf{x}}=\gamma_{\mu, \mathbf{y}}$.

Lemma C.7 Let $F, G: \Delta(\mathcal{X}) \rightrightarrows \mathbb{R}$ be $S M E$ support rules. For any $\mathbf{x}, \mathbf{y} \in \mathcal{X}$, if $d(\mathbf{x}, \mathbf{y}) \leq$ 2 , then $\mathcal{B}_{\mathbf{x}, \mathbf{y}}^{F} \subseteq \operatorname{cl}\left(\mathcal{C} \backslash \mathcal{C}_{\mathbf{x}}^{G}\right)$. 
Proof: Let $\mathbf{b} \in \mathcal{B}_{\mathbf{x}, \mathbf{y}}^{F}$; we must show that $\mathbf{b} \in \operatorname{cl}\left(\mathcal{C} \backslash \mathcal{C}_{\mathbf{x}}^{G}\right)$. Find $\mu \in \Delta(\mathcal{X})$ such that $\mathbf{b}=\tilde{\mu}$.

Claim 1: $\quad \mathrm{x} \bullet \mathrm{b}=\mathrm{y} \bullet \mathrm{b}$.

Proof: First note that

$$
\begin{aligned}
& (\mathrm{x} \bullet \mathrm{b}<\mathrm{y} \bullet \mathrm{b}) \underset{\overline{(\diamond)}}{\Longrightarrow}(\mathrm{x} \notin \operatorname{SME}(\mathcal{X}, \mu)) \\
& \Longrightarrow(\mathrm{x} \notin F(\mathbf{b})) \Longleftrightarrow\left(\mathrm{b} \notin \mathcal{C}_{\mathbf{x}}^{F}\right) \text {. }
\end{aligned}
$$

Here, $(\diamond)$ is by Lemma C.6, while $(*)$ is because $F$ is supermajoritarian efficient.

Conversely, if $\mathbf{b} \in \mathcal{C}_{\mathbf{x}}^{F}$, then $\mathbf{x} \bullet \mathbf{b} \geq \mathbf{y} \bullet \mathbf{b}$. Likewise, if $\mathbf{b} \in \mathcal{C}_{\mathbf{y}}^{F}$, then $\mathbf{x} \bullet \mathbf{b} \leq \mathbf{y} \bullet \mathbf{b}$. Thus, if $\mathbf{b} \in \mathcal{B}_{\mathbf{x}, \mathbf{y}}^{F}=\mathcal{C}_{\mathbf{x}}^{F} \cap \mathcal{C}_{\mathbf{y}}^{F}$, then $\mathbf{x} \bullet \mathbf{b}=\mathbf{y} \bullet \mathbf{b}$.

Now, for any $r \in[0,1]$, let $\mathbf{b}^{r}:=r \mathbf{y}+(1-r) \mathbf{b}$. Then $\mathbf{x} \bullet \mathbf{b}^{r}<\mathbf{y} \bullet \mathbf{b}^{r}$. Identical reasoning to statement (C5) implies that $\mathbf{b}^{r} \notin \mathcal{C}_{\mathbf{x}}^{G}$ (because $G$ is also supermajoritarian efficent); hence $\mathbf{b}^{r} \in \mathcal{C} \backslash \mathcal{C}_{\mathbf{x}}^{G}$ for all $r>0$. But $\mathbf{b}^{r} \underset{r \rightarrow 0}{\longrightarrow} \mathbf{b}$. Thus, $\mathbf{b} \in \operatorname{cl}\left(\mathcal{C} \backslash \mathcal{C}_{\mathbf{x}}^{G}\right)$, as desired.

Proof of Proposition C.2. " $\Longrightarrow "$ follows immediately from Proposition C.3.

"£" follows from Lemmas C.7 and C.3.

\section{Appendix D: Facts about additive rules}

This appendix collects some technical results about additive judgement aggregation rules, which are often used in the proofs in Appendix E. For example, the next lemma is used in the proofs of Theorem 3.4(a) and Lemma D.2.

Lemma D.1 Suppose $\phi:[-1,1] \longrightarrow \mathbb{L}$ is odd. Let $\mathcal{X}$ be a judgement space, and let $\mu \in$ $\Delta(\mathcal{X})$. For any $\mathbf{x} \in \mathcal{X}$, let $\mathcal{M}(\mu, \mathbf{x}):=\left\{k \in \mathcal{K} ; x_{k} \widetilde{\mu}_{k} \geq 0\right\}$. Then $F_{\phi}(\mathcal{X}, \mu)=$ $\underset{\mathbf{x} \in \mathcal{X}}{\operatorname{argmax}} \sum_{k \in \mathcal{M}(\mathbf{x}, \mu)} \phi\left|\widetilde{\mu}_{k}\right|$.

Proof: For any $\mathbf{x}, \mathbf{y} \in \mathcal{X}$, define

$$
\begin{aligned}
\mathcal{K}_{0} & :=\left\{k \in \mathcal{K} ; \widetilde{\mu}_{k}=0\right\}, \\
\mathcal{K}_{++} & :=\mathcal{M}(\mu, \mathbf{x}) \cap \mathcal{M}(\mu, \mathbf{y}) \backslash \mathcal{K}_{0}=\left\{k \in \mathcal{K} ; x_{k} \widetilde{\mu}_{k}=y_{k} \widetilde{\mu}_{k}>0\right\}, \\
\mathcal{K}_{+-} & :=\mathcal{M}(\mu, \mathbf{x}) \cap \mathcal{M}(\mu, \mathbf{y})^{\complement} \backslash \mathcal{K}_{0}=\left\{k \in \mathcal{K} ; x_{k} \widetilde{\mu}_{k}=-y_{k} \widetilde{\mu}_{k}>0\right\}, \\
\mathcal{K}_{-+} & :=\mathcal{M}(\mu, \mathbf{x})^{\complement} \cap \mathcal{M}(\mu, \mathbf{y}) \backslash \mathcal{K}_{0}=\left\{k \in \mathcal{K} ; y_{k} \widetilde{\mu}_{k}=-x_{k} \widetilde{\mu}_{k}>0\right\}, \\
\text { and } \quad \mathcal{K}_{--} & :=\mathcal{M}(\mu, \mathbf{x})^{\complement} \cap \mathcal{M}(\mu, \mathbf{y})^{\complement} \backslash \mathcal{K}_{0}=\left\{k \in \mathcal{K} ; x_{k} \widetilde{\mu}_{k}=y_{k} \widetilde{\mu}_{k}<0\right\} .
\end{aligned}
$$


Then $\mathcal{K}=\mathcal{K}_{0} \sqcup \mathcal{K}_{++} \sqcup \mathcal{K}_{+-} \sqcup \mathcal{K}_{-+} \sqcup \mathcal{K}_{--}$. Thus,

$$
\begin{aligned}
& \sum_{k \in \mathcal{K}} \phi\left(x_{k} \widetilde{\mu}_{k}\right)-\sum_{k \in \mathcal{K}} \phi\left(y_{k} \widetilde{\mu}_{k}\right)=\sum_{k \in \mathcal{K}}\left(\phi\left(x_{k} \widetilde{\mu}_{k}\right)-\phi\left(y_{k} \widetilde{\mu}_{k}\right)\right) \\
& \overline{\overline{(\diamond)}} \sum_{k \in \mathcal{K}_{+-}}\left(\phi\left(x_{k} \widetilde{\mu}_{k}\right)-\phi\left(y_{k} \widetilde{\mu}_{k}\right)\right)+\sum_{k \in \mathcal{K}_{-+}}\left(\phi\left(x_{k} \widetilde{\mu}_{k}\right)-\phi\left(y_{k} \widetilde{\mu}_{k}\right)\right) \\
& \overline{\overline{(*)}} \sum_{k \in \mathcal{K}_{+-}}\left(\phi\left(\left|\widetilde{\mu}_{k}\right|\right)-\phi\left(-\left|\widetilde{\mu}_{k}\right|\right)\right)+\sum_{k \in \mathcal{K}_{-+}}\left(\phi\left(-\left|\widetilde{\mu}_{k}\right|\right)-\phi\left(\left|\widetilde{\mu}_{k}\right|\right)\right) \\
& \overline{\overline{(\dagger)}} \sum_{k \in \mathcal{K}_{+-}}\left(\phi\left|\widetilde{\mu}_{k}\right|+\phi\left|\widetilde{\mu}_{k}\right|\right)+\sum_{k \in \mathcal{K}_{-+}}\left(-\phi\left|\widetilde{\mu}_{k}\right|-\phi\left|\widetilde{\mu}_{k}\right|\right) \\
& =2 \sum_{k \in \mathcal{K}_{+-}} \phi\left|\widetilde{\mu}_{k}\right|-2 \sum_{k \in \mathcal{K}_{-+}} \phi\left|\widetilde{\mu}_{k}\right| \\
& \overline{\overline{(\diamond)}} 2 \sum_{k \in \mathcal{K}_{+-} \sqcup \mathcal{K}_{++} \sqcup \mathcal{K}_{0}} \phi\left|\widetilde{\mu}_{k}\right|-2 \sum_{k \in \mathcal{K}_{-+} \sqcup \mathcal{K}_{++} \sqcup \mathcal{K}_{0}} \phi\left|\widetilde{\mu}_{k}\right| \\
& =2 \sum_{k \in \mathcal{M}(\mu, \mathbf{x})} \phi\left|\widetilde{\mu}_{k}\right|-2 \sum_{k \in \mathcal{M}(\mu, \mathbf{y})} \phi\left|\widetilde{\mu}_{k}\right| .
\end{aligned}
$$

Here, both $(\diamond)$ are because $\phi\left(x_{k} \widetilde{\mu}_{k}\right)=\phi\left(y_{k} \widetilde{\mu}_{k}\right)$ for all $k \in \mathcal{K}_{++} \sqcup \mathcal{K}_{--} \sqcup \mathcal{K}_{0}$. Next, $(*)$ is by the definitions of $\mathcal{K}_{+-}$and $\mathcal{K}_{-+}$. Finally $(\dagger)$ is because $\phi$ is odd. Thus,

$$
\begin{aligned}
\left(\sum_{k \in \mathcal{K}} \phi\left(x_{k} \widetilde{\mu}_{k}\right) \geq \sum_{k \in \mathcal{K}} \phi\left(y_{k} \widetilde{\mu}_{k}\right)\right) & \Longleftrightarrow\left(\sum_{k \in \mathcal{M}(\mu, \mathbf{x})} \phi\left|\widetilde{\mu}_{k}\right| \geq \sum_{k \in \mathcal{M}(\mu, \mathbf{y})} \phi\left|\widetilde{\mu}_{k}\right|\right) . \\
\text { Thus, } \underset{\mathbf{x} \in \mathcal{X}}{\operatorname{argmax}}\left(\sum_{k \in \mathcal{K}} \phi\left(x_{k} \widetilde{\mu}_{k}\right)\right) & =\underset{\mathbf{x} \in \mathcal{X}}{\operatorname{argmax}} \sum_{k \in \mathcal{M}(\mathbf{x}, \mu)} \phi\left|\widetilde{\mu}_{k}\right| .
\end{aligned}
$$

Let $\Gamma$ be the space of all functions on $(0,1]$ of the form $\sum_{n=1}^{N} a_{n} \mathbf{1}_{\left(q_{n}, r_{n}\right]}$, where $a_{n} \in \mathbb{R}$ and $0 \leq q_{n}<r_{n} \leq 1$ for all $n \in[1 \ldots N]$. Endow $\Gamma$ with the supremum norm: $\|\gamma\|_{\infty}:=$ $\sup |\gamma(r)|$ for all $\gamma \in \Gamma$. Let $\Gamma_{+}$be the set of all elements of $\Gamma$ which are nonnegative $r \in(0,1]$

everywhere A positive linear functional on $\Gamma$ is a linear function $P: \Gamma \longrightarrow \mathbb{R}$ which is $\|\bullet\|_{\infty^{-}}$ continuous, such that $P(\gamma) \geq 0$ for all $\gamma \in \Gamma_{+}$. Let $\Gamma_{+}^{*}$ be the set of all positive linear functionals on $\Gamma$. The next result is used in the proofs of Propositions 1.1(b) and 3.5(a), as well as Theorem 2.1(a).

Lemma D.2 Let $\Gamma_{+}^{*}$ be the set of positive linear functionals on $\Gamma$. Let $\Phi_{\mathrm{OND}}$ be the set of odd, nondecreasing functions from $[-1,1]$ into $\mathbb{R}$.

(a) There is a bijective correspondence between $\Phi_{\mathrm{OND}}$ and $\Gamma_{+}^{*}$ defined as follows. Given any $\phi \in \Phi_{\mathrm{OND}}$, define $\phi^{*}: \Gamma \longrightarrow \mathbb{R}$ by first defining $\phi^{*}\left(\mathbf{1}_{(q, r]}\right):=\phi(r)-\phi(q)$ for all $q<r \in[0,1]$, and then extending $\phi^{*}$ to all of $\Gamma$ by linearity. Then $\phi^{*} \in \Gamma_{+}^{*}$.

To invert this map, suppose $P \in \Gamma_{+}^{*}$. Define $\phi:[0,1] \longrightarrow \mathbb{R}_{+}$by setting $\phi(q):=$ $P\left(\mathbf{1}_{(0, q]}\right)$ for all $q \in[0,1]$, and $\phi(q):=-\phi(-q)$ for all $q \in[-1,0]$. Then $\phi \in \Phi_{\mathrm{OND}}$, and $\phi^{*}=P$. 
Let $\mathcal{X}$ be a judgement space, and let $\mu \in \Delta(\mathcal{X})$.

(b) For all $\mathbf{x} \in \mathcal{X}$ and any $\phi \in \Phi_{\mathrm{OND}}$, we have $\gamma_{\mu, \mathbf{x}} \in \Gamma_{+}$, and $\phi^{*}\left(\gamma_{\mathbf{x}, \mu}\right)=\sum_{k \in \mathcal{M}(\mathbf{x}, \mu)} \phi\left|\widetilde{\mu}_{k}\right|$.

(c) Thus, for any $\phi \in \Phi_{\mathrm{OND}}$, we have $F_{\phi}(\mathcal{X}, \mu)=\underset{\mathbf{x} \in \mathcal{X}}{\operatorname{argmax}} \phi^{*}\left(\gamma_{\mu, \mathbf{x}}\right)$.

Proof: (a) Given $\phi \in \Phi_{\mathrm{OND}}$, it is easy to check that $\phi^{*} \in \Gamma_{+}^{*}$.

Conversely, let $P \in \Gamma_{+}^{*}$ and define $\phi$ as above. By construction, $\phi$ is odd and $\phi(0)=0$. To see that $\phi$ is nondecreasing, let $q<r \in(0,1]$. Then

$$
\phi(r)=P\left(\mathbf{1}_{(0, r]}\right)=P\left(\mathbf{1}_{(0, q]}+\mathbf{1}_{(q, r]}\right)=P\left(\mathbf{1}_{(0, q]}\right)+P\left(\mathbf{1}_{(q, r]}\right) \underset{(*)}{\geq} P\left(\mathbf{1}_{(0, q]}\right)=\phi(q) .
$$

(*) because $P\left(\mathbf{1}_{(q, r]}\right) \geq 0$ because $P$ is positive, and $\mathbf{1}_{(q, r]} \in \Gamma_{+}$. Equation (D1) also shows that $P\left(\mathbf{1}_{(q, r]}\right)=\phi(r)-\phi(q)$ for $q<r \in(0,1]$; thus $P=\phi^{*}$.

(b) For any $\mathbf{x} \in \mathcal{X}$, we have

$$
\begin{aligned}
\gamma_{\mathbf{x}, \mu} & =\sum_{k \in \mathcal{M}(\mathbf{x}, \mu)} \mathbf{1}_{\left(0,\left|\widetilde{\mu}_{k}\right|\right]} . \\
\text { Thus, } \phi^{*}\left(\gamma_{\mathbf{x}, \mu}\right) & =\sum_{k \in \mathcal{M}(\mathbf{x}, \mu)} \phi^{*}\left(\mathbf{1}_{\left(0,\left|\widetilde{\mu}_{k}\right|\right]}\right)=\sum_{k \in \mathcal{M}(\mathbf{x}, \mu)} \phi\left|\widetilde{\mu}_{k}\right| .
\end{aligned}
$$

(c) follows from part (b) and Lemma D.1.

The proofs of Proposition 1.1(b) and Theorem 3.4(a) are based on separation arguments involving finite-dimensional polyhedra. Fix $Q \in \mathbb{N}$, and let $\mathbb{R}_{+}^{Q}:=\left\{\mathbf{r} \in \mathbb{R}^{Q} ; r_{q} \geq 0\right.$, for all $q \in[1 \ldots Q]\}$ be the non-negative orthant. The next lemma slightly strengthens a well-known fact about Pareto optimality.

Lemma D.3 Let $Q \in \mathbb{N}$. Let $\mathcal{P} \subset \mathbb{R}^{Q}$ be a closed, convex polyhedron in which $\mathbf{0}$ is Pareto optimal (i.e. $\mathcal{P} \cap \mathbb{R}_{+}^{Q}=\{\mathbf{0}\}$ ). Then there exists an all-positive vector $\mathbf{v} \in \mathbb{R}_{+}^{Q}$ such that $\mathbf{v} \bullet \mathbf{p} \leq 0$ for all $\mathbf{p} \in \mathcal{P}$.

Proof: Let $\mathcal{T}:=\mathcal{P}-\mathbb{R}_{+}^{Q}$; then $\mathcal{T}$ is a closed, convex polyhedron.

Claim 1: $\mathcal{T} \cap \mathbb{R}_{+}^{Q}=\{0\}$.

Proof: (by contradiction) If $\mathbf{t} \in \mathcal{T}$, then $\mathbf{t}=\mathbf{p}-\mathbf{r}$ for some $\mathbf{p} \in \mathcal{P}$ and $\mathbf{r} \in \mathbb{R}_{+}^{Q}$. Thus $\mathbf{p}=\mathbf{t}+\mathbf{r}$. Thus, if $\mathbf{t} \in \mathbb{R}_{+}^{Q} \backslash\{\mathbf{0}\}$, then $\mathbf{p} \in \mathbb{R}_{+}^{Q} \backslash\{\mathbf{0}\}$ also, contradicting the Pareto optimality of $\mathbf{0}$.

$\diamond$ Claim 1

Thus, $\mathbf{0}$ lies on the boundary of the polyhedron $\mathcal{T}$, so it is contained in some face. Let $\mathcal{F}$ be the minimal-dimension face of $\mathcal{T}$ containing $\mathbf{0}$. Thus, if $\mathcal{S} \subseteq \mathbb{R}^{Q}$ is the linear subspace spanned by $\mathcal{F}$, then $\mathcal{F}=\mathcal{S} \cap \mathcal{T}$, and there exists some $\epsilon>0$ such that $\mathcal{B}_{\epsilon} \cap \mathcal{S} \subset \mathcal{F}$, where $\mathcal{B}_{\epsilon} \subset \mathbb{R}^{Q}$ is the $\epsilon$-ball around $\mathbf{0}$. 
$\mathcal{F}$ is a face of $\mathcal{T}$, so there exists some $\mathbf{v} \in \mathbb{R}^{\mathcal{K}}$ such that $\mathcal{F}=\operatorname{argmax}_{\mathbf{t} \in \mathcal{T}}(\mathbf{v} \bullet \mathbf{t})$. Clearly $\mathbf{v} \bullet \mathbf{0}=0$; hence $\mathbf{v} \bullet \mathbf{f}=0$ for all $\mathbf{f} \in \mathcal{F}$, while $\mathbf{v} \bullet \mathbf{t}<0$ for all $\mathbf{t} \in \mathcal{T} \backslash \mathcal{F}$. Thus, if $\mathcal{V} \subset \mathbb{R}^{Q}$ is the hyperplane orthogonal to $\mathbf{v}$, then $\mathcal{F}=\mathcal{T} \cap \mathcal{V}$.

Claim 2: $\quad \mathbf{v} \bullet \mathbf{r}>0$ for all $\mathbf{r} \in \mathbb{R}_{+}^{Q} \backslash\{0\}$.

Proof: For any $\mathbf{r} \in \mathbb{R}_{+}^{Q} \backslash\{\mathbf{0}\}$, we have $-\mathbf{r} \in \mathcal{T}$, so $\mathbf{v} \bullet(-\mathbf{r}) \leq 0$, so $\mathbf{v} \bullet \mathbf{r} \geq 0$. It remains to show that $\mathbf{v} \bullet \mathbf{r} \neq 0$. By contradiction, suppose $\mathbf{v} \bullet \mathbf{r}=0$. Then $\mathbf{r} \in \mathcal{V}$. Thus, $-\mathbf{r} \in \mathcal{V}$. But $-\mathbf{r} \in \mathcal{T}$ also, so $-\mathbf{r} \in \mathcal{T} \cap \mathcal{V}=\mathcal{F}=\mathcal{T} \cap \mathcal{S}$. But $\mathcal{F}$ contains a relative neighbourhood around $\mathbf{0}$ in the subspace $\mathcal{S}$, so if $-\mathbf{r} \in \mathcal{F}$, then there exists some $\epsilon>0$ such that $\epsilon \mathbf{r} \in \mathcal{F}$; hence $\epsilon \mathbf{r} \in \mathcal{T}$. But $\epsilon \mathbf{r} \in \mathbb{R}_{+}^{Q} \backslash\{\mathbf{0}\}$, so this contradicts Claim 1 . $\diamond$ Claim 2

For any $q \in[1 \ldots Q]$, let $\mathbf{e}_{q}=(0,0, \ldots, 0,1,0, \ldots, 0)$ be the $q$ th unit vector. Then $\mathbf{e}_{q} \in \mathbb{R}_{+}^{Q}$. Thus, Claim 2 says that $\mathbf{v} \bullet \mathbf{e}_{q}>0$. But this means that $v_{q}>0$, as desired.

\section{Appendix E: Proofs of the main results}

The proofs of Proposition 1.1 and Theorems 2.1, 3.4, and E.7(b) all depend upon Proposition 3.5. Thus, we will prove Proposition 3.5 before anything else. Here is a preliminary lemma.

Lemma E.1 Let $\mathcal{X}$ be a judgement space, and let $F: \Delta(\mathcal{X}) \rightrightarrows \mathcal{X}$ be a judgement aggregation rule. If $F$ is monotone, then $F$ is generically single-valued on $\Delta(\mathcal{X})$.

Proof: Suppose $F$ is monotone. Fix $\mathbf{y} \in \mathcal{X}$. Define

$$
\Delta_{\mathbf{y}}:=\{\mu \in \Delta(\mathcal{X}) ; \mathbf{y} \in F(\mu)\} \text { and } \Delta_{\mathbf{y}}^{o}:=\{\mu \in \Delta(\mathcal{X}) ; F(\mu)=\{\mathbf{y}\}\} .
$$

Claim 1: $\quad$ Suppose $\operatorname{int}\left(\Delta_{\mathbf{y}}\right) \neq \emptyset$. Then there is an open subset $\mathcal{O}_{\mathbf{y}} \subset \Delta(\mathcal{X})$ such that $\mathcal{O}_{\mathbf{y}} \subset \Delta_{\mathbf{y}}^{o}$ but $\mathcal{O}_{\mathbf{y}}$ is dense in $\Delta_{\mathbf{y}}$.

Proof: Let $\Delta_{\mathbf{x}}^{*}:=\left\{\mu \in \Delta_{\mathbf{y}} ; \mu(\mathbf{x})>0\right.$ for all $\left.\mathbf{x} \in \mathcal{X}\right\}$. Then $\Delta_{\mathbf{x}}^{*}$ is a dense open subset of $\Delta_{\mathbf{y}}$. For any $\mu \in \Delta_{\mathbf{y}}^{*}$, define $\mathcal{O}_{\mu}:=\left\{\mu^{\prime} \in \Delta(\mathcal{X}) ; \mu^{\prime}(\mathbf{y})>\mu(\mathbf{y})\right.$ while $\mu^{\prime}(\mathbf{x})<\mu(\mathbf{x})$, for all $\mathbf{x} \in \mathcal{X} \backslash\{\mathbf{y}\}\}$. Then $\mathcal{O}_{\mu}$ is an open subset of $\Delta(\mathcal{X})$. Furthermore, $\mathcal{O}_{\mu} \subset \Delta_{\mathbf{y}}^{o}$ because $F$ is monotone, and any element of $\mathcal{O}_{\mu}$ is more supportive than $\mu$ of $\mathbf{y}$. Note that $\mu$ is a cluster point of $\mathcal{O}_{\mu}$. Now let $\mathcal{O}_{\mathbf{y}}:=\bigcup_{\mu \in \Delta_{\mathbf{y}}^{*}} \mathcal{O}_{\mu}$. Then every point in $\Delta_{\mathbf{y}}^{*}$ is a cluster point of $\mathcal{O}_{\mathbf{y}}$. Thus, $\mathcal{O}_{\mathbf{y}}$ is dense in $\Delta_{\mathbf{y}}$, because $\Delta_{\mathbf{y}}^{*}$ is dense in $\Delta_{\mathbf{y}} . \quad \diamond$ claim 1

Let $\mathcal{Y}:=\left\{\mathbf{y} \in \mathcal{X} ; \operatorname{int}\left(\Delta_{\mathbf{y}}\right) \neq \emptyset\right\}$. Let $\Delta^{\prime}:=\bigcup_{\mathbf{y} \in \mathcal{Y}} \Delta_{\mathbf{y}}$. Then $\Delta^{\prime}$ is dense in $\Delta(\mathcal{X})$ (because its compliment has empty interior). Let $\mathcal{O}:=\bigsqcup_{\mathbf{y} \in \mathcal{Y}} \mathcal{O}_{\mathbf{y}}$. Then $\mathcal{O}$ is an open subset of $\Delta(\mathcal{X})$, and $F$ is single-valued on $\mathcal{O}$ by definition. It remains to show that $\mathcal{O}$ is dense in $\Delta(\mathcal{X})$, and for this, it suffices to show that $\mathcal{O}$ is dense in $\Delta^{\prime}$. Let $\mu \in \Delta^{\prime}$; then $\mu \in \Delta_{\mathbf{y}}$ for some $\mathbf{y} \in \mathcal{Y}$. Thus, $\mu$ is a cluster point of $\mathcal{O}_{\mathbf{y}}$ by Claim 1 , and thus, a cluster point of $\mathcal{O}$. Thus, $\mathcal{O}$ is dense in $\Delta^{\prime}$. 
Proof of Proposition 3.5 (a) Suppose $\phi$ is strictly increasing. We must show that $F_{\phi}(\mathcal{X}, \mu) \subseteq$ $\operatorname{SSME}(\mathcal{X}, \mu)$. Contrapositively, let $\mathbf{y} \in \mathcal{X}$, and suppose $\mathbf{y} \notin \operatorname{SSME}(\mathcal{X}, \mu)$; we will show that $\mathbf{y} \notin F_{\phi}(\mathcal{X}, \mu)$.

Define $\phi^{*}: \Gamma \longrightarrow \mathbb{R}$ as in Lemma D.2(a). For any $\rho \in \Delta(\mathcal{X})$, we have $\gamma_{\mu, \rho} \in \Gamma_{+}$, and

$$
\phi^{*}\left(\gamma_{\mu, \rho}\right) \quad \overline{\overline{(\diamond)}} \phi^{*}\left(\sum_{\mathbf{x} \in \mathcal{X}} \rho(\mathbf{x}) \cdot \gamma_{\mu, \mathbf{x}}\right)=\sum_{\mathbf{x} \in \mathcal{X}} \rho(\mathbf{x}) \cdot \phi^{*}\left(\gamma_{\mu, \mathbf{x}}\right) \text {. }
$$

where $(\diamond)$ is by eqn.(8) defining $\gamma_{\mu, \rho}$.

If $\mathbf{y} \notin \operatorname{SSME}(\mathcal{X}, \mu)$, then there exists some $\rho \in \Delta(\mathcal{X})$ such that $\rho{ }_{\mu} \mathbf{y}$. Thus, $\gamma_{\mu, \rho} \geq \gamma_{\mu, \mathbf{y}}$, and there exist $q_{1}<q_{2} \in[0,1]$ such that $\gamma_{\mu, \rho}(r)>\gamma_{\mu, \mathbf{y}}(r)$ for all $r \in\left[q_{1}, q_{2}\right]$. For any $\epsilon>0$, let $\mathcal{R}_{\epsilon}:=\left\{r \in\left[q_{1}, q_{2}\right] ; \gamma_{\mu, \rho}(r)-\gamma_{\mu, \mathbf{y}}(r)>\epsilon\right\}$. If $\epsilon$ is small enough, then there exist $r_{1}<r_{2} \in\left[q_{1}, q_{2}\right]$ such that $\left(r_{1}, r_{2}\right] \in \mathcal{R}_{\epsilon}$; thus, $\gamma_{\mu, \rho}-\gamma_{\mu, \mathbf{y}} \geq \epsilon \cdot \mathbf{1}_{\left(r_{1}, r_{2}\right]}$. Thus,

$$
\begin{aligned}
\phi^{*}\left(\gamma_{\mu, \rho}\right)-\phi^{*}\left(\gamma_{\mu, \mathbf{y}}\right) & =\phi^{*}\left(\gamma_{\mu, \rho}-\gamma_{\mu, \mathbf{y}}\right) \underset{(*)}{\geq} \phi^{*}\left(\epsilon \mathbf{1}_{\left(r_{1}, r_{2}\right]}\right) \\
& =\epsilon \cdot \phi^{*}\left(\mathbf{1}_{\left(r_{1}, r_{2}\right]}\right)=\epsilon\left(\phi\left(r_{2}\right)-\phi\left(r_{1}\right)\right) \underset{(\dagger)}{>} 0 .
\end{aligned}
$$

Here, $(*)$ is because $\phi^{*}$ is positive and $\gamma_{\mu, \rho}-\gamma_{\mu, \mathbf{y}} \geq \epsilon \cdot \mathbf{1}_{\left(r_{1}, r_{2}\right]}$, while $(\dagger)$ is because $\phi$ is strictly increasing. Combine (E1) and (E2) to get

$$
\sum_{\mathbf{x} \in \mathcal{X}} \rho(\mathbf{x}) \cdot \phi^{*}\left(\gamma_{\mu, \mathbf{x}}\right)>\phi^{*}\left(\gamma_{\mu, \mathbf{y}}\right) .
$$

Thus, there is some $\mathbf{x} \in \mathcal{X}$ such that $\phi^{*}\left(\gamma_{\mu, \mathbf{x}}\right)>\phi^{*}\left(\gamma_{\mu, \mathbf{y}}\right)$. Thus, $\mathbf{y} \notin F_{\phi}(\mathcal{X}, \mu)$, by Lemma D.2(c).

(b) Let $\mu \in \Delta(\mathcal{X})$ and $\mathbf{y} \in F_{\phi}(\mu)$. Let $\mu^{\prime} \in \Delta(\mathcal{X})$ be more supportive than $\mu$ of $\mathbf{y}$; we must show that $F_{\phi}\left(\mu^{\prime}\right)=\{\mathbf{y}\}$. By negating certain coordinates of $\mathcal{X}$ if necessary, we can assume without loss of generality that $\mathbf{y}=\mathbf{1}$.

Claim 1: For all $k \in \mathcal{K}$, if there exists $\mathbf{x} \in \mathcal{X}$ with $x_{k}=-1$, then $\widetilde{\mu}_{k}^{\prime}>\widetilde{\mu}_{k}$.

Proof: Recall that $\mathcal{X}(\mu):=\{\mathbf{x} \in \mathcal{X} ; \mu(\mathbf{x})>0\}$. If $\mu^{\prime}$ is more supportive than $\mu$ of $\mathbf{y}$, then $\mathcal{X}\left(\mu^{\prime}\right) \subseteq \mathcal{X}(\mu)$. Let $\mathcal{X}_{-}:=\left\{\mathbf{x} \in \mathcal{X}(\mu) ; x_{k}=-1\right\}$ and $\mathcal{X}_{+}:=\{\mathbf{x} \in \mathcal{X}(\mu) ; \mathbf{x} \neq \mathbf{y}$ but $\left.x_{k}=1\right\}$. Then

$$
\begin{aligned}
& \left(\widetilde{\mu}_{k}^{\prime}-\widetilde{\mu}_{k}\right) \overline{\overline{(\diamond)}} \sum_{\mathbf{x} \in \mathcal{X}(\mu)}\left(\mu^{\prime}(\mathbf{x})-\mu(\mathbf{x})\right) x_{k} \\
& =\left(\mu^{\prime}(\mathbf{y})-\mu(\mathbf{y})\right) y_{k}+\sum_{\mathbf{x} \in \mathcal{X}_{-}}\left(\mu^{\prime}(\mathbf{x})-\mu(\mathbf{x})\right) x_{k}+\sum_{\mathbf{x} \in \mathcal{X}_{+}}\left(\mu^{\prime}(\mathbf{x})-\mu(\mathbf{x})\right) x_{k} . \\
& \overline{\overline{(+)}}\left(\mu^{\prime}(\mathbf{y})-\mu(\mathbf{y})\right)-\sum_{\mathbf{x} \in \mathcal{X}_{-}}\left(\mu^{\prime}(\mathbf{x})-\mu(\mathbf{x})\right)+\sum_{\mathbf{x} \in \mathcal{X}_{+}}\left(\mu^{\prime}(\mathbf{x})-\mu(\mathbf{x})\right) \\
& \underset{(*)}{>}\left(\mu^{\prime}(\mathbf{y})-\mu(\mathbf{y})\right)+\sum_{\mathbf{x} \in \mathcal{X}_{-}}\left(\mu^{\prime}(\mathbf{x})-\mu(\mathbf{x})\right)+\sum_{\mathbf{x} \in \mathcal{X}_{+}}\left(\mu^{\prime}(\mathbf{x})-\mu(\mathbf{x})\right) \\
& =\sum_{\mathbf{x} \in \mathcal{X}(\mu)}\left(\mu^{\prime}(\mathbf{x})-\mu(\mathbf{x})\right)=1-1=0 .
\end{aligned}
$$


Here, $(\diamond)$ is by defining equation $(2),(\dagger)$ is by definition of $\mathcal{X}_{-}$and $\mathcal{X}_{+}$, and $(*)$ is because $\mathcal{X}_{-} \neq \emptyset$ (by the hypothesis of the claim), while $\mu^{\prime}(\mathbf{x})-\mu(\mathbf{x})<0$ for all $\mathbf{x} \in \mathcal{X}_{-}$ by the definition of $\mu^{\prime}$.

$\diamond$ Claim 1

Now, for any $\mathbf{x} \in \mathcal{X} \backslash\{\mathbf{y}\}$, we will show that $\mathbf{x} \notin F\left(\mu^{\prime}\right)$. For all $k \in \mathcal{K}_{ \pm}(\mathbf{x}, \mathbf{y})$, we have $y_{k}=1$ and $x_{k}=-1$, while Claim 1 says $\widetilde{\mu}_{k}^{\prime}>\widetilde{\mu}_{k}$. Thus,

$$
(\mathbf{y}-\mathbf{x}) \bullet \phi\left(\widetilde{\mu}^{\prime}\right)=\sum_{k \in \mathcal{K}_{ \pm}(\mathbf{x}, \mathbf{y})} 2 \phi\left(\widetilde{\mu}_{k}^{\prime}\right) \underset{(\diamond)}{>} \sum_{k \in \mathcal{K}_{ \pm}(\mathbf{x}, \mathbf{y})} 2 \phi\left(\widetilde{\mu}_{k}\right)=(\mathbf{y}-\mathbf{x}) \bullet \phi(\widetilde{\mu}) \underset{(*)}{\geq} 0,
$$

and thus, $\mathbf{x} \notin F_{\phi}\left(\mu^{\prime}\right)$. Here, $(\diamond)$ is because $\phi$ is increasing, and $(*)$ is because $\mathbf{y} \in F_{\phi}(\mu)$. We conclude that $\mathbf{x} \notin F_{\phi}\left(\mu^{\prime}\right)$ for all $\mathbf{x} \in \mathcal{X} \backslash\{\mathbf{y}\}$; thus, $F\left(\widetilde{\mu}^{\prime}\right)=\{\mathbf{y}\}$, as desired.

(c) follows immediately from part (b) and Lemma E.1.

\section{Proofs from $\S 1$}

Proof of Proposition 1.1. (a) $\operatorname{SSME}(\mathcal{X}, \mu)$ is nonempty because it is obtained by maximizing over a finite set. For any $\mathbf{x}, \mathbf{y} \in \mathcal{X}$, observe that

$$
(\mathcal{M}(\mathbf{x}, \mu) \supset \mathcal{M}(\mathbf{y}, \mu)) \Longrightarrow\left(\mathbf{x}_{\mu}^{\triangleright} \mathbf{y}\right) \Longrightarrow\left(\delta_{\mathbf{x}} \underset{\mu}{\triangleright} \delta_{\mathbf{y}}\right) .
$$

Thus, if $\delta_{\mathbf{y}}$ is undominated in $\left(\Delta(\mathcal{X}),{ }_{\mu}\right)$, then $\mathbf{y}$ must be undominated in $\left(\mathcal{X}, \triangleright_{\mu}\right)$. Likewise if $\mathbf{y}$ is undominated in $(\mathcal{X}, \underset{\mu}{\triangleright})$, then $\mathbf{y}$ must be Condorcet efficient in $\mathcal{X}$.

To see that, in general, $\operatorname{SME}(\mathcal{X}, \mu) \subsetneq \operatorname{Cond}(\mathcal{X}, \mu)$, consider the Slater rule:

$$
\operatorname{Slater}(\mathcal{X}, \mu):=\underset{\mathbf{x} \in \mathcal{X}}{\operatorname{argmax}}|\mathcal{M}(\mathbf{x}, \mu)| \text {. }
$$

It is easy to see that $\operatorname{Slater}(\mathcal{X}, \mu) \subseteq \operatorname{Cond}(\mathcal{X}, \mu)$. However, it is also possible to construct examples where $\operatorname{Slater}(\mathcal{X}, \mu) \not \subset \operatorname{SME}(\mathcal{X}, \mu)$ (Nehring and Pivato, 2011b). Thus, Cond $(\mathcal{X}, \mu) \backslash \operatorname{SME}(\mathcal{X}, \mu) \neq \emptyset$.

It is also possible to construct an example where $\operatorname{SSME}(\mathcal{X}, \mu) \subsetneq \operatorname{SME}(\mathcal{X}, \mu)$; see Nehring and Pivato (2011b)

(b) "ᄃ" follows immediately from Proposition 3.5(a).

"卫" Let $\mathcal{Q}(\mu):=\left\{\left|\widetilde{\mu}_{k}\right| ; k \in \mathcal{K}\right\}$, and write $\mathcal{Q}=\left\{q_{1}, q_{2}, \ldots, q_{N}\right\}$, where $0 \leq q_{1}<q_{2}<$ $\cdots<q_{N} \leq 1$. Define $q_{0}:=0$. Let $\Gamma$ be the space of functions defined above Lemma D.2. Define a linear injective map $f_{\mu}: \mathbb{R}^{N} \longrightarrow \Gamma$ by

$$
f_{\mu}\left(r_{1}, \ldots, r_{N}\right):=\sum_{n=1}^{N} r_{n} \mathbf{1}_{\left(q_{n-1}, q_{n}\right]}=r_{1} \mathbf{1}_{\left(0, q_{1}\right]}+r_{2} \mathbf{1}_{\left(q_{1}, q_{2}\right]}+\cdots+r_{N} \mathbf{1}_{\left(q_{N-1}, q_{N}\right]} .
$$

Let $\Gamma_{\mu}:=f_{\mu}\left[\mathbb{R}^{N}\right]$ (a subspace of $\Gamma$ ); then $\gamma_{\mu, \rho} \in \Gamma_{\mu}$ for all $\rho \in \Delta(\mathcal{X})$. Let $g_{\mu}:=f_{\mu}^{-1}$ : $\Gamma_{\mu} \longrightarrow \mathbb{R}^{N}$, and then define $G_{\mu}: \Delta(\mathcal{X}) \longrightarrow \mathbb{R}^{N}$ by $G_{\mu}(\rho):=g_{\mu}\left(\gamma_{\mu, \rho}\right)$. Let $\mathcal{P}_{\mu}:=G_{\mu}[\Delta(\mathcal{X})] ;$ 
then $\mathcal{P}_{\mu}$ is a convex, compact polyhedron in $\mathbb{R}^{N}$. For all $\mathbf{x} \in \mathcal{X}$, let $G_{\mu}(\mathbf{x}):=g_{\mu}\left(\gamma_{\mu, \mathbf{x}}\right) \in$ $\mathcal{P}_{\mu}$. Let " $<$ " be the Pareto relation on $\mathbb{R}^{N}$. Fix $\mathbf{x} \in \mathcal{X}$. We have

$$
\left(\mathrm{x}_{\mu}^{\triangleleft} \rho\right) \Longleftrightarrow\left(G_{\mu}(\mathbf{x})<G_{\mu}(\rho)\right), \quad \text { for all } \rho \in \Delta(\mathcal{X}) .
$$

Thus, $(\mathrm{x} \in \operatorname{SSME}(\mathcal{X}, \mu)) \Longleftrightarrow\left(G_{\mu}(\mathbf{x})\right.$ is Pareto optimal in $\left.\mathcal{P}_{\mu}\right)$.

Lemma D.3 now yields a strictly positive vector $\mathbf{v} \in \mathbb{R}_{+}^{N}$ such that

$$
\mathbf{v} \bullet G_{\mu}(\mathbf{x}) \quad \geq \mathbf{v} \bullet \mathbf{p}, \quad \text { for all } \mathbf{p} \in \mathcal{P}_{\mu} .
$$

Construct $\phi \in \Phi_{I}$ such that $\phi\left(q_{n}\right)-\phi\left(q_{n-1}\right)=v_{n}$ for all $n \in[1 \ldots N]$. (For example, $\phi$ could be piecewise linear, with vertices at $q_{1}, \ldots, q_{N}$.) Let $\phi^{*}: \Gamma \longrightarrow \mathbb{R}$ be the positive linear functional defined by $\phi$, as in Lemma D.2(a). Then for all $\mathbf{y} \in \mathcal{X}$, if $G_{\mu}(\mathbf{y})=\mathbf{p} \in \mathcal{P}_{\mu}$, then

$$
\begin{aligned}
\phi^{*}\left(\gamma_{\mu, \mathbf{y}}\right) & =\phi^{*}\left(f_{\mu}(\mathbf{p})\right)=\phi^{*}\left(\sum_{n=1}^{N} p_{n} \mathbf{1}_{\left(q_{n-1}, q_{n}\right]}\right)=\sum_{n=1}^{N} p_{n} \phi^{*}\left(\mathbf{1}_{\left(q_{n-1}, q_{n}\right]}\right) \\
= & \sum_{n=1}^{N} p_{n}\left(\phi\left(q_{n}\right)-\phi\left(q_{n-1}\right)\right)=\sum_{n=1}^{N} p_{n} v_{n}=\mathbf{v} \bullet \mathbf{p}=\mathbf{v} \bullet G_{\mu}(\mathbf{y}) . \\
\text { Thus, } & \left(\mathbf{v} \bullet G_{\mu}(\mathbf{x}) \geq \mathbf{v} \bullet G_{\mu}(\mathbf{y})\right) \Longleftrightarrow\left(\phi^{*}\left(\gamma_{\mu, \mathbf{x}}\right) \geq \phi^{*}\left(\gamma_{\mu, \mathbf{y}}\right)\right) .
\end{aligned}
$$

Thus, equation (E4) implies that $\phi^{*}\left(\gamma_{\mu, \mathbf{x}}\right) \geq \phi^{*}\left(\gamma_{\mu, \mathbf{y}}\right)$ for all $\mathbf{y} \in \mathcal{X}$. Thus, Lemma D.2(c) says that $\mathrm{x} \in F_{\phi}(\mathcal{X}, \mu)$, as desired.

\section{Proofs from $\S 3$}

Proof of Lemma 3.2. (a) Define $\widehat{\phi}(r):=\phi(r)-\phi(-r)$ for all $r \in[-1,1]$. Then $\widehat{\phi}$ is odd. Let $\mathbf{x}, \mathbf{y} \in \mathcal{X}$. Recall that $\mathcal{K}_{ \pm}(\mathbf{x}, \mathbf{y}):=\left\{k \in \mathcal{K} ; x_{k} \neq y_{k}\right\}$. Then

$$
\begin{aligned}
& \sum_{k \in \mathcal{K}} \phi\left(x_{k} \widetilde{\mu}_{k}\right)-\sum_{k \in \mathcal{K}} \phi\left(y_{k} \widetilde{\mu}_{k}\right)=\sum_{k \in \mathcal{K}_{ \pm}(\mathbf{x}, \mathbf{y})}\left(\phi\left(x_{k} \widetilde{\mu}_{k}\right)-\phi\left(y_{k} \widetilde{\mu}_{k}\right)\right) \\
& \overline{\overline{(*)}} \sum_{k \in \mathcal{K}_{ \pm}(\mathbf{x}, \mathbf{y})}\left(\phi\left(x_{k} \widetilde{\mu}_{k}\right)-\phi\left(-x_{k} \widetilde{\mu}_{k}\right)\right) \quad \overline{\overline{(\dagger)}} \sum_{k \in \mathcal{K}_{ \pm}(\mathbf{x}, \mathbf{y})} \widehat{\phi}\left(x_{k} \widetilde{\mu}_{k}\right) \\
& \overline{\overline{(\ddagger)}} \frac{1}{2} \sum_{k \in \mathcal{K}_{ \pm}(\mathbf{x}, \mathbf{y})}\left(\widehat{\phi}\left(x_{k} \widetilde{\mu}_{k}\right)-\widehat{\phi}\left(-x_{k} \widetilde{\mu}_{k}\right)\right) \overline{\overline{(*)}} \frac{1}{2} \sum_{k \in \mathcal{K}_{ \pm}(\mathbf{x}, \mathbf{y})}\left(\widehat{\phi}\left(x_{k} \widetilde{\mu}_{k}\right)-\widehat{\phi}\left(y_{k} \widetilde{\mu}_{k}\right)\right) \\
& =\frac{1}{2}\left(\sum_{k \in \mathcal{K}} \widehat{\phi}\left(x_{k} \widetilde{\mu}_{k}\right)-\sum_{k \in \mathcal{K}} \widehat{\phi}\left(y_{k} \widetilde{\mu}_{k}\right)\right) .
\end{aligned}
$$

Here $(*)$ is because $y_{k}=-x_{k}$ for all $k \in \mathcal{K}_{ \pm}(\mathbf{x}, \mathbf{y})$. Meanwhile $(\dagger)$ is simply the definition of $\widehat{\phi}$, and ( $\ddagger$ ) is because $\widehat{\phi}$ is odd, so $\widehat{\phi}(r)-\widehat{\phi}(-r)=2 \widehat{\phi}(r)$. 
Equation (E5) implies that

$$
\left(\sum_{k \in \mathcal{K}} \phi\left(x_{k} \widetilde{\mu}_{k}\right) \geq \sum_{k \in \mathcal{K}} \phi\left(y_{k} \widetilde{\mu}_{k}\right)\right) \Longleftrightarrow\left(\sum_{k \in \mathcal{K}} \widehat{\phi}\left(x_{k} \widetilde{\mu}_{k}\right) \geq \sum_{k \in \mathcal{K}} \widehat{\phi}\left(y_{k} \widetilde{\mu}_{k}\right)\right) .
$$

Thus, $F_{\phi}(\mathcal{X}, \mu)=F_{\widehat{\phi}}(\mathcal{X}, \mu)$.

(b) If $\phi$ is odd, then for any $\mu \in \Delta(\mathcal{X}), \mathbf{x} \in \mathcal{X}$, and $k \in \mathcal{K}$, we have $\phi\left(x_{k} \widetilde{\mu}_{k}\right)=x_{k} \phi\left(\widetilde{\mu}_{k}\right)$ (because $\left.x_{k}= \pm 1\right)$. Thus, $\sum_{k \in \mathcal{K}} \phi\left(x_{k} \cdot \widetilde{\mu}_{k}\right)=\sum_{k \in \mathcal{K}} x_{k} \phi\left(\widetilde{\mu}_{k}\right)=\mathbf{x} \bullet \phi(\widetilde{\mu})$. Thus, $\underset{\mathbf{x} \in \mathcal{X}}{\operatorname{argmax}}\left(\sum_{k \in \mathcal{K}} \phi\left(x_{k} \widetilde{\mu}_{k}\right)\right)=$ $\underset{\mathbf{x} \in \mathcal{X}}{\operatorname{argmax}} \mathbf{x} \bullet \phi(\widetilde{\mu})$.

Fix $\omega \in \Omega$. Let $\mathcal{Q}:=\mathcal{Q}_{\omega} \cap[0,1]$; then $\mathbb{R}^{\mathcal{Q}}$ is a finite-dimensional vector space (because $\mathcal{Q}_{\omega} \subset[-1,1]$ is a finite set). Let $\mathcal{X}$ be a judgement space, and let $\mu \in \Delta(\mathcal{X})$. For any $\mathbf{x} \in \mathcal{X}$, we define $\mathbf{g}(\mathbf{x}, \mu) \in \mathbb{R}^{\mathcal{Q}}$ by setting

$$
g_{q}(\mathbf{x}, \mu):=\frac{\gamma_{\mu, \mathbf{x}}(q)}{|\mathcal{K}|}=\frac{\#\left\{k \in \mathcal{K} ; x_{k} \widetilde{\mu}_{k} \geq q\right\}}{|\mathcal{K}|}, \quad \text { for all } q \in \mathcal{Q} .
$$

We then define $\mathcal{D}(\mathbf{x} ; \mathcal{X}, \mu):=\{\mathbf{g}(\mathbf{y}, \mu)-\mathbf{g}(\mathbf{x}, \mu) ; \mathbf{y} \in \mathcal{X}\} \subseteq \mathbb{R}^{\mathcal{Q}}$. If $F: \Delta_{\omega}(\mathcal{X}) \rightrightarrows \mathcal{X}$ is a judgement aggregation rule, then define

$$
\mathcal{D}_{\omega}(F, \mathcal{X}):=\bigcup_{\mu \in \Delta_{\omega}(\mathcal{X})} \bigcup_{\mathbf{x} \in F(\mathcal{X}, \mu)} \mathcal{D}(\mathbf{x} ; \mathcal{X}, \mu) \subseteq \mathbb{R}^{\mathcal{Q}}
$$

Finally, let $\mathfrak{X}$ be a judgement monoid. If $F$ is a judgement aggregation rule on $\Delta_{\omega}(\mathfrak{X})$, then define $\mathcal{D}_{\omega}(F, \mathfrak{X}):=\bigcup_{\mathcal{X} \in \mathfrak{X}} \mathcal{D}_{\omega}(F, \mathcal{X}) \subseteq \mathbb{R}^{\mathcal{Q}}$.

Lemma E.2 $F$ is $S M E$ on $\Delta_{\omega}(\mathfrak{X})$ if and only if $\mathcal{D}_{\omega}(F, \mathfrak{X}) \cap \mathbb{R}_{+}^{\mathcal{Q}}=\{\mathbf{0}\}$.

Proof: For any $\mathcal{X} \in \mathfrak{X}, \mu \in \Delta_{\omega}(\mathcal{X})$, and $\mathrm{x} \in \mathcal{X}$, we have

$$
\begin{aligned}
(\mathrm{x} & \in \operatorname{SME}(\mathcal{X}, \mu)) \\
& \Longleftrightarrow\left(\nexists \mathbf{y} \in \mathcal{X} \text { with } g_{q}(\mathbf{y}, \mu) \geq g_{q}(\mathbf{x}, \mu) \text { for all } q \in \mathcal{Q}, \text { and } \mathbf{g}(\mathbf{y}, \mu) \neq \mathbf{g}(\mathbf{x}, \mu)\right) \\
& \Longleftrightarrow\left((\mathbf{g}(\mathbf{y}, \mu)-\mathbf{g}(\mathbf{x}, \mu)) \notin \mathbb{R}_{+}^{\mathcal{Q}} \backslash\{\mathbf{0}\} \text { for all } \mathbf{y} \in \mathcal{X}\right) \\
& \Longleftrightarrow\left(\mathcal{D}(\mathbf{x} ; \mathcal{X}, \mu) \cap \mathbb{R}_{+}^{\mathcal{Q}}=\{\mathbf{0}\}\right) .
\end{aligned}
$$

Thus, $F$ is supermajoritarian efficient on $\Delta_{\omega}(\mathfrak{X})$ if and only if $\mathcal{D}(\mathbf{x} ; \mathcal{X}, \mu) \cap \mathbb{R}_{+}^{\mathcal{Q}}=\{\mathbf{0}\}$ whenever $\mathrm{x} \in F(\mathcal{X}, \mu)$ for some $(\mathcal{X}, \mu) \in \Delta_{\omega}(\mathfrak{X})$. The claim follows.

Let $\mathcal{P}_{\omega}(F, \mathfrak{X})$ be the closure of $\mathcal{D}_{\omega}(F, \mathfrak{X})$. 
Lemma E.3 Let $\mathfrak{X}$ be a judgement monoid.

(a) If $F$ is a separable judgement aggregation rule on $\Delta(\mathfrak{X})$, then $\mathcal{P}_{\omega}(F, \mathfrak{X})$ is a closed, convex subset of $\mathbb{R}^{\mathcal{Q}}$.

(b) Also, if $\mathfrak{X}$ is finitely generated, then $\mathcal{P}_{\omega}(F, \mathfrak{X})$ is a convex polyhedron in $\mathbb{R}^{\mathcal{Q}}$.

Proof: (a) For any $(\mathcal{X}, \mu) \in \Delta(\mathfrak{X})$, define $\mathcal{D}(F, \mathcal{X}, \mu):=\bigcup_{\mathbf{x} \in F(\mathcal{X}, \mu)} \mathcal{D}(\mathbf{x} ; \mathcal{X}, \mu)$. (Thus,

$$
\left.\mathcal{D}_{\omega}(F, \mathcal{X})=\bigcup_{\mu \in \Delta_{\omega}(\mathcal{X})} \mathcal{D}_{\omega}(F, \mathcal{X}, \mu) .\right)
$$

Claim 1: Let $\mathcal{X}_{1}, \mathcal{X}_{2} \in \mathfrak{X}$, and suppose $\mathcal{X}_{1} \subseteq\{ \pm 1\}^{\mathcal{K}_{1}}$ and $\mathcal{X}_{2} \subseteq\{ \pm 1\}^{\mathcal{K}_{2}}$ for some finite sets $\mathcal{K}_{1}, \mathcal{K}_{2}$. If $\mathcal{X}:=\mathcal{X}_{1} \times \mathcal{X}_{2}$, then $\mathcal{X} \subseteq\{ \pm 1\}^{\mathcal{K}}$, where $\mathcal{K}:=\mathcal{K}_{1} \sqcup \mathcal{K}_{2}$. Let $s_{1}:=\left|\mathcal{K}_{1}\right| /|\mathcal{K}|$ and $s_{2}:=\left|\mathcal{K}_{2}\right| /|\mathcal{K}|\left(\right.$ so $\left.s_{1}+s_{2}=1\right)$.

(a) For any $\mu_{1} \in \Delta\left(\mathcal{X}_{1}\right)$ and $\mu_{2} \in \Delta\left(\mathcal{X}_{2}\right)$, there exists a profile $\mu=\mu_{1} \otimes \mu_{2} \in \Delta(\mathcal{X})$ such that $\mu^{(1)}=\mu_{1}$ and $\mu^{(2)}=\mu_{2}$.

(b) For any $\mathbf{x}=\left(\mathbf{x}^{1}, \mathbf{x}^{2}\right) \in \mathcal{X}$, we have $\mathbf{g}(\mathbf{x}, \mu)=s_{1} \mathbf{g}\left(\mathbf{x}^{1}, \mu^{(1)}\right)+s_{2} \mathbf{g}\left(\mathbf{x}^{2}, \mu^{(2)}\right)$.

(c) $\mathcal{D}(F, \mathcal{X}, \mu)=\left\{s_{1} \mathbf{d}_{1}+s_{2} \mathbf{d}_{2} ; \mathbf{d}_{1} \in \mathcal{D}\left(F, \mathcal{X}_{1}, \mu^{(1)}\right)\right.$ and $\left.\mathbf{d}_{2} \in \mathcal{D}\left(F, \mathcal{X}_{2}, \mu^{(2)}\right)\right\}$.

Proof: (a) For all $\mathbf{x}_{1} \in \mathcal{X}_{1}$ and $\mathbf{x}_{2} \in \mathcal{X}_{2}$, recall that $\left(\mathbf{x}_{1}, \mathbf{x}_{2}\right) \in \mathcal{X}$. Define $\mu \in \Delta(\mathcal{X})$ by setting $\mu\left(\mathbf{x}_{1}, \mathbf{x}_{2}\right):=\mu_{1}\left(\mathbf{x}_{1}\right) \cdot \mu_{2}\left(\mathbf{x}_{2}\right)$, for all $\mathbf{x}_{1} \in \mathcal{X}_{1}$ and $\mathbf{x}_{2} \in \mathcal{X}_{2}$.

For any $\mathbf{x}_{1} \in \mathcal{X}_{1}$, we have

$$
\begin{aligned}
\mu^{(1)}\left(\mathbf{x}_{1}\right) \overline{\overline{(*)}} \sum_{\mathbf{x}_{2} \in \mathcal{X}_{2}} \mu\left(\mathbf{x}_{1}, \mathbf{x}_{2}\right) & =\sum_{\mathbf{x}_{2} \in \mathcal{X}_{2}} \mu_{1}\left(\mathbf{x}_{1}\right) \cdot \mu_{2}\left(\mathbf{x}_{2}\right)=\mu_{1}\left(\mathbf{x}_{1}\right) \cdot \sum_{\mathbf{x}_{2} \in \mathcal{X}_{2}} \mu_{2}\left(\mathbf{x}_{2}\right)=\mu_{1}\left(\mathbf{x}_{1}\right), \\
\text { because } \sum_{\mathbf{x}_{2} \in \mathcal{X}_{2}} \mu_{2}\left(\mathbf{x}_{2}\right) & =1 .
\end{aligned}
$$

Here $(*)$ is by defining equation (10). Thus, $\mu^{(1)}=\mu_{1}$. Likewise, $\mu^{(2)}=\mu_{2}$.

(b) Note that $\widetilde{\mu}=\left(\widetilde{\mu}^{(1)}, \widetilde{\mu}^{(2)}\right) \in \mathbb{R}^{\mathcal{K}}$. Thus, for both $n \in\{1,2\}$ and all $k \in \mathcal{K}_{n}$, we have $x_{k} \cdot \tilde{\mu}_{k}=x_{k}^{n} \cdot \tilde{\mu}_{k}^{(n)}$. Thus, for any $q \in(0,1]$, we have

$$
\begin{aligned}
\left\{k \in \mathcal{K} ; x_{k} \cdot \widetilde{\mu}_{k} \geq q\right\} & =\left\{k \in \mathcal{K}_{1} ; x_{k}^{1} \cdot \widetilde{\mu}_{k}^{(1)} \geq q\right\} \sqcup\left\{k \in \mathcal{K}_{2} ; x_{k}^{2} \cdot \widetilde{\mu}_{k}^{(2)} \geq q\right\} . \\
\text { Thus, }|\mathcal{K}| \cdot g_{q}(\mathbf{x}, \mu) & =\left|\left\{k \in \mathcal{K} ; x_{k} \cdot \widetilde{\mu}_{k} \geq q\right\}\right| \\
& =\left|\left\{k \in \mathcal{K}_{1} ; x_{k}^{1} \cdot \widetilde{\mu}_{k}^{(1)} \geq q\right\}\right|+\left|\left\{k \in \mathcal{K}_{2} ; x_{k}^{2} \cdot \widetilde{\mu}_{k}^{(2)} \geq q\right\}\right| \\
& =\left|\mathcal{K}_{1}\right| \cdot g_{q}\left(\mathbf{x}^{1}, \mu^{(1)}\right)+\left|\mathcal{K}_{2}\right| \cdot g_{q}\left(\mathbf{x}^{2}, \mu^{(2)}\right) .
\end{aligned}
$$

now divide both sides by $|\mathcal{K}|$ to prove part (b). 
(c) Let $\mathbf{x}:=\left(\mathbf{x}_{1}, \mathbf{x}_{2}\right)$ and $\mathbf{y}:=\left(\mathbf{y}_{1}, \mathbf{y}_{2}\right)$ be elements of $\mathcal{X}$; Thus, $\mathbf{x}_{1}, \mathbf{y}_{1} \in \mathcal{X}_{1}$ and $\mathbf{x}_{2}, \mathbf{y}_{2} \in$ $\mathcal{X}_{2}$. Let $\mathbf{d}:=\mathbf{g}(\mathbf{y}, \mu)-\mathbf{g}(\mathbf{x}, \mu)$, and let $\mathbf{d}_{n}:=\mathbf{g}\left(\mathbf{y}_{n}, \mu^{(n)}\right)-\mathbf{g}\left(\mathbf{x}_{n}, \mu^{(n)}\right)$, for $n \in\{1,2\}$. Part (b) implies that

$$
\begin{aligned}
\mathbf{d} & =\mathbf{g}(\mathbf{y}, \mu)-\mathbf{g}(\mathbf{x}, \mu) \\
& =s_{1} \mathbf{g}\left(\mathbf{y}_{1}, \mu^{(1)}\right)-s_{1} \mathbf{g}\left(\mathbf{x}_{1}, \mu^{(1)}\right)+s_{2} \mathbf{g}\left(\mathbf{y}_{2}, \mu^{(2)}\right)-s_{2} \mathbf{g}\left(\mathbf{x}_{2}, \mu^{(2)}\right)=s_{1} \mathbf{d}_{1}+s_{2} \mathbf{d}_{2} .
\end{aligned}
$$

But

$$
\begin{aligned}
(\mathrm{d} \in \mathcal{D}(F, \mathcal{X}, \mu)) & \Longleftrightarrow(\mathrm{x} \in F(\mathcal{X}, \mu)) \Longleftrightarrow\left(\mathrm{x}_{n} \in F\left(\mathcal{X}_{n}, \mu^{(n)}\right) \text { for } n=1,2\right) \\
& \Longleftrightarrow\left(\mathbf{d}_{n} \in \mathcal{D}\left(F, \mathcal{X}_{n}, \mu^{(n)}\right) \text { for } n=1,2\right)
\end{aligned}
$$

where $(*)$ is because $F$ is separable.

For any $N \in \mathbb{N}$, let $\Delta_{N}:=\left\{\mathrm{s} \in \mathbb{R}_{+}^{N} ; \sum_{n=1}^{N} s_{n}=1\right\}$ be the $N$-simplex.

Claim 2: $\quad$ Let $N \geq 2$, and let $\mathbf{d}_{1}, \ldots, \mathbf{d}_{N} \in \mathcal{D}_{\omega}(F, \mathfrak{X})$. There exists a dense subset $\mathcal{S} \subseteq \Delta_{N}$ such that, for any $\mathbf{s}=\left(s_{1}, \ldots, s_{N}\right) \in \mathcal{S}$, we have $\sum_{n=1}^{N} s_{n} \mathbf{d}_{n} \in \mathcal{D}_{\omega}(F, \mathfrak{X})$.

Proof: For all $n \in[1 \ldots N]$, there exists $\left(\mathcal{X}_{n}, \mu_{n}\right) \in \Delta_{\omega}(\mathfrak{X})$ such that $\mathbf{d}_{n} \in \mathcal{D}\left(F, \mathcal{X}_{n}, \mu_{n}\right)$. Let $K_{n}=\left|\mathcal{K}_{n}\right|$ for $n \in[1 \ldots N]$. Define

$$
\mathcal{S}:=\left\{\frac{\left(m_{1} K_{1}, m_{2} K_{2}, \ldots, m_{N} K_{N}\right)}{m_{1} K_{1}+\cdots+m_{N} K_{N}} ; m_{1}, \ldots, m_{N} \in \mathbb{N}\right\} .
$$

Then $\mathcal{S}$ is a dense subset of $\Delta_{N}$.

Let $\mathbf{s} \in \mathcal{S}$ and let $m_{1}, \ldots, m_{N}$ be as in eqn.(E6). For all $n \in[1 \ldots N]$, let $\mathcal{X}_{n}^{m_{n}}$ denote the $m_{n}$-fold Cartesian power of $\mathcal{X}_{n}$, and let

$$
\mu_{n}^{\otimes m_{n}}:=\underbrace{\mu_{n} \otimes \mu_{n} \otimes \cdots \otimes \mu_{n}}_{m_{n}}
$$

where the operator " $\otimes$ " is as defined in Claim 2(a). Finally, let $\mathcal{X}:=\mathcal{X}_{1}^{m_{1}} \times \mathcal{X}_{2}^{m_{2}} \times$ $\cdots \times \mathcal{X}_{N}^{m_{N}}$, and let $\mu:=\mu_{1}^{\otimes m_{1}} \otimes \mu_{2}^{\otimes m_{2}} \otimes \cdots \otimes \mu_{N}^{\otimes m_{N}}$. Then $\mathcal{X} \in \mathfrak{X}$ (because $\mathfrak{X}$ is a monoid) and $\mu \in \Delta(\mathcal{X})$, and inductive application of Claim 2(c) implies that $s_{1} \mathbf{d}_{1}+\cdots+s_{N} \mathbf{d}_{N} \in \mathcal{D}(F, \mathcal{X}, \mu) ;$ thus, $s_{1} \mathbf{d}_{1}+\cdots+s_{N} \mathbf{d}_{N} \in \mathcal{D}_{\omega}(F, \mathfrak{X})$.

$\diamond$ Claim 2

Now, $\mathcal{P}_{\omega}(\mathfrak{X}, F)$ is the closure of $\mathcal{D}_{\omega}(\mathfrak{X}, F)$. Thus, for any $\mathbf{d}_{1}, \ldots, \mathbf{d}_{N} \in \mathcal{D}_{\omega}(\mathfrak{X}, F)$ and any $\left(s_{1}, \ldots, s_{N}\right) \in \Delta_{N}$, Claim 2 implies that $s_{1} \mathbf{d}_{1}+\cdots+s_{N} \mathbf{d}_{N} \in \mathcal{P}_{\omega}(\mathfrak{X}, F)$. Thus $\mathcal{P}_{\omega}(\mathfrak{X}, F)$ contains the convex hull $\operatorname{conv}\left[\mathcal{D}_{\omega}(\mathfrak{X}, F)\right]$. Thus, $\mathcal{D}_{\omega}(\mathfrak{X}, F) \subseteq \operatorname{conv}\left[\mathcal{D}_{\omega}(\mathfrak{X}, F)\right] \subseteq$ $\mathcal{P}_{\omega}(\mathfrak{X}, F)=\overline{\mathcal{D}_{\omega}(\mathfrak{X}, F)}$; hence $\mathcal{P}_{\omega}(\mathfrak{X}, F)=\overline{\operatorname{conv}\left[\mathcal{D}_{\omega}(\mathfrak{X}, F)\right]}$, so $\mathcal{P}_{\omega}(\mathfrak{X}, F)$ is the closure of a convex set, and thus, itself convex. 
(b) Let $\mathcal{X}_{1}, \ldots, \mathcal{X}_{N}$ be judgement spaces, and suppose $\mathfrak{X}:=\left\langle\mathcal{X}_{1}, \ldots, \mathcal{X}_{N}\right\rangle$. Define

$$
\mathcal{E}:=\bigcup_{n=1}^{N} \bigcup_{\mu \in \Delta_{\omega}\left(\mathcal{X}_{n}\right)} \mathcal{D}\left(F, \mathcal{X}_{n}, \mu\right)
$$

Then $\mathcal{E}$ is a finite set, because for each $n \in[1 \ldots N]$, the set $\Delta_{\omega}\left(\mathcal{X}_{n}\right)$ is finite (because $\omega$ is finitary), and for each $\mu \in \Delta_{\omega}\left(\mathcal{X}_{n}\right)$, the set $\mathcal{D}\left(F, \mathcal{X}_{n}, \mu\right)$ is finite (because $\mathcal{X}_{n}$ is finite). Thus, $\operatorname{conv}(\mathcal{E})$ is the convex hull of a finite set, and thus, a closed, convex polyhedron in $\mathbb{R}^{\mathcal{Q}}$.

For any $\mathcal{X} \in \mathfrak{X}$, if $\mathcal{X}=\mathcal{X}_{1}^{m_{1}} \times \mathcal{X}_{2}^{m_{2}} \times \cdots \times \mathcal{X}_{N}^{m_{N}}$, then Claim 1(c) implies that every element of $\mathcal{D}_{\omega}(F, \mathcal{X})$ is a rational convex combination of elements from $\mathcal{E}$. Thus, $\mathcal{D}_{\omega}(F, \mathcal{X}) \subseteq$ $\operatorname{conv}(\mathcal{E})$. This holds for all $\mathcal{X} \in \mathfrak{X}$; thus, $\mathcal{D}_{\omega}(F, \mathfrak{X}) \subseteq \operatorname{conv}(\mathcal{E})$. Since conv $(\mathcal{E})$ is closed, we deduce that $\operatorname{cl}\left(\mathcal{D}_{\omega}(F, \mathfrak{X})\right) \subseteq \operatorname{conv}(\mathcal{E})$.

Conversely, Claim 2 shows that every convex combination of elements from $\mathcal{E}$ lies in $\operatorname{cl}\left(\mathcal{D}_{\omega}(F, \mathfrak{X})\right)$. Thus, $\operatorname{conv}(\mathcal{E}) \subseteq \operatorname{cl}\left(\mathcal{D}_{\omega}(F, \mathfrak{X})\right)$.

Thus, $\operatorname{conv}(\mathcal{E})=\operatorname{cl}\left(\mathcal{D}_{\omega}(F, \mathfrak{X})\right)=\mathcal{P}_{\omega}(F, \mathfrak{X})$. Thus, $\mathcal{P}_{\omega}(F, \mathfrak{X})$ is a convex polyhedron.

Proof of Theorem 3.4. (a) "£" If $\phi$ is strictly increasing, then for all $\mathcal{X} \in \mathfrak{X}$ and $\mu \in \Delta_{\omega}(\mathcal{X})$, Proposition 3.5(a) says $F_{\phi}(\mu) \subseteq \operatorname{SME}(\mathcal{X}, \mu)$, and thus, $F(\mu) \subseteq \operatorname{SME}(\mathcal{X}, \mu)$. Thus, $F$ is supermajoritarian efficient on $\Delta_{\omega}(\mathfrak{X})$.

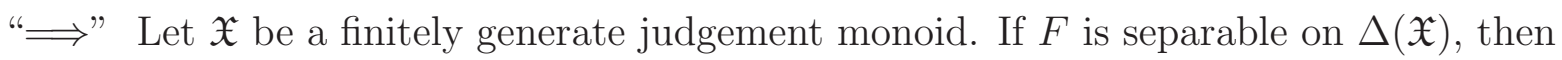
Lemma E.3(b) says $\mathcal{P}_{\omega}(F, \mathfrak{X})$ is a closed, convex polyhedron in $\mathbb{R}^{\mathcal{Q}}$. If $F$ is SME, then Lemma E.2 implies that $\mathcal{P}_{\omega}(F, \mathfrak{X}) \cap \mathbb{R}_{+}^{\mathcal{Q}}=\{\mathbf{0}\}$. Thus, Lemma D.3 yields an all-positive vector $\mathbf{v} \in \mathbb{R}_{+}^{\mathcal{Q}}$ such that $\mathbf{v} \bullet \mathbf{p} \leq 0$ for all $\mathbf{p} \in \mathcal{P}_{\omega}(F, \mathfrak{X})$.

Now we define $\phi: \mathcal{Q}_{\omega} \longrightarrow \mathbb{R}$ as follows: for all $r \in \mathcal{Q}_{\omega}$, define

$$
\phi(r):=\sum_{\substack{q \in \mathcal{Q} \\ q \leq r}} v_{q} \text { if } r \geq 0 \text {, and } \phi(r):=-\phi(-r) \text { if } r \leq 0 \text {. }
$$

Thus, $\phi$ is odd by construction, and $\phi$ is strictly increasing on $\mathcal{Q}_{\omega}$, because $v_{q}>0$ for all $q \in \mathcal{Q}_{\omega}$.

Let $\mathcal{X} \in \mathfrak{X}$ and $\mu \in \Delta_{\omega}(\mathcal{X})$. For any $\mathbf{x} \in \mathcal{X}$, recall $\mathcal{M}(\mu, \mathbf{x}):=\left\{k \in \mathcal{K} ; x_{k} \widetilde{\mu}_{k} \geq 0\right\}$.

Claim 1: For any $\mathbf{x} \in \mathcal{X}$, we have $|\mathcal{K}| \cdot \mathbf{v} \bullet \mathbf{g}(\mathbf{x}, \mu)=\sum_{k \in \mathcal{M}(\mathbf{x}, \mu)} \phi\left|\widetilde{\mu}_{k}\right|$.

Proof: For any $r, q \in[-1,1]$, define $\delta_{q}^{r}:=1$ if $r \geq q$, whereas $\delta_{q}^{r}:=0$ if $r<q$. Then

$$
\begin{aligned}
|\mathcal{K}| \cdot \mathbf{v} \bullet \mathbf{g}(\mathbf{x}, \mu) & =|\mathcal{K}| \cdot \sum_{q \in \mathcal{Q}} v_{q} \cdot g_{q}(\mathbf{x}, \mu)=\sum_{q \in \mathcal{Q}} v_{q} \cdot \#\left\{k \in \mathcal{K} ; x_{k} \widetilde{\mu}_{k} \geq q\right\} \\
& =\sum_{q \in \mathcal{Q}} v_{q} \sum_{k \in \mathcal{K}} \delta_{q}^{x_{k} \widetilde{\mu}_{k}}=\sum_{k \in \mathcal{K}} \sum_{q \in \mathcal{Q}} v_{q} \delta_{q}^{x_{k} \widetilde{\mu}_{k}} \\
& =\sum_{k \in \mathcal{K}} \sum_{\substack{q \in \mathcal{Q} \\
q \leq x_{k}}} v_{q} \overline{\bar{\mu}} \sum_{\substack{k \in \mathcal{K} \\
x_{k} \widetilde{\mu}_{k} \geq 0}} \phi\left(x_{k} \widetilde{\mu}_{k}\right)=\sum_{k \in \mathcal{M}(\mathbf{x}, \mu)} \phi\left|\widetilde{\mu}_{k}\right| .
\end{aligned}
$$


Here, $(*)$ is by definition (E7).

We must show that $F(\mathcal{X}, \mu) \subseteq F_{\phi}(\mathcal{X}, \mu)$. To see this, let $\mathbf{x} \in F(\mathcal{X}, \mu)$. For any other $\mathbf{y} \in \mathcal{X}$, we have $\mathbf{g}(\mathbf{y}, \mu)-\mathbf{g}(\mathbf{x}, \mu) \in \mathcal{D}_{\omega}(F, \mathfrak{X}) \subseteq \mathcal{P}_{\omega}(F, \mathfrak{X})$, so $\mathbf{v} \bullet(\mathbf{g}(\mathbf{y}, \mu)-\mathbf{g}(\mathbf{x}, \mu)) \leq 0$, and hence $\mathbf{v} \bullet \mathbf{g}(\mathbf{y}, \mu) \leq \mathbf{v} \bullet \mathbf{g}(\mathbf{x}, \mu)$. Thus, $F(\mathcal{X}, \mu) \subseteq \underset{\mathbf{x} \in \mathcal{X}}{\operatorname{argmax}}(\mathbf{v} \bullet \mathbf{g}(\mu, \mathbf{x}))$. Thus, Claim 1 and Lemma D.1 imply that $F(\mathcal{X}, \mu) \subseteq F_{\phi}(\mathcal{X}, \mu)$, as desired.

(b) "£" exactly the same proof as part (a).

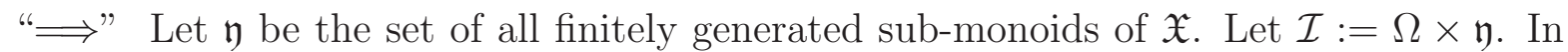
effect, $\mathcal{I}$ is the set of possible 'inputs' to the construction in part (a).

For any finite collection $\mathcal{T}:=\left\{\left(\mathcal{X}_{1}, \mu_{1}\right),\left(\mathcal{X}_{2}, \mu_{2}\right), \ldots,\left(\mathcal{X}_{N}, \mu_{N}\right)\right\} \subset \Delta(\mathfrak{X})$, define $\mathcal{I}_{\mathcal{T}}:=$ $\left\{(\omega, \mathfrak{Y}) \in \mathcal{I} ; \quad \mathcal{X}_{n} \in \mathfrak{Y}\right.$ and $\mu_{n} \in \Delta_{\omega}\left(\mathcal{X}_{n}\right)$ for all $\left.n \in[1 \ldots N]\right\}$. Then let $\mathfrak{E}:=\{\mathcal{J} \subseteq \mathcal{I}$; $\mathcal{I}_{\mathcal{T}} \subseteq \mathcal{J}$ for some nonempty finite $\left.\mathcal{T} \subset \Delta(\mathfrak{X})\right\}$.

Claim 2: $\quad \mathfrak{E}$ is a free filter.

Proof: We must check axioms (F0)-(F2).

(F0) Let $\mathcal{T}:=\left\{\left(\mathcal{X}_{1}, \mu_{1}\right),\left(\mathcal{X}_{2}, \mu_{2}\right), \ldots,\left(\mathcal{X}_{N}, \mu_{N}\right)\right\} \subset \Delta(\mathfrak{X})$. Fix $\omega \in \Omega$, and suppose $\operatorname{supp}(\omega)=[1 \ldots W]$. Let $M:=W \cdot\left|\mathcal{X}_{1}\right| \cdot\left|\mathcal{X}_{2}\right| \cdots\left|\mathcal{X}_{N}\right|$, and let $\beta:[1 \ldots M] \longrightarrow[1 \ldots W] \times$ $\mathcal{X}_{1} \times \mathcal{X}_{2} \times \cdots \times \mathcal{X}_{N}$ be some bijection. Define $\omega^{\prime} \in \Omega$ as follows: for all $m \in[1 \ldots M]$, if $\beta(m)=\left(w, \mathbf{x}_{1}, \ldots, \mathbf{x}_{N}\right)$, then let $\omega^{\prime}(m):=\omega(w) \cdot \mu_{1}\left(\mathbf{x}_{1}\right) \cdots \mu_{N}\left(\mathbf{x}_{N}\right)$. Let $\mathfrak{Y} \subset$ $\mathfrak{X}$ be any finitely generated sub-monoid containing the finitely generated monoid $\left\langle\mathcal{X}_{1}, \ldots, \mathcal{X}_{N}\right\rangle$. Then it is easy to check that $\left(\mathcal{X}_{n}, \mu_{n}\right) \in \Delta_{\omega^{\prime}}(\mathfrak{Y})$ for all $n \in[1 \ldots N]$. Thus, $\left(\omega^{\prime}, \mathfrak{Y}\right) \in \mathcal{I}_{\mathcal{T}}$.

We can repeat this construction for any $\omega \in \Omega$; thus, $\mathcal{I}_{\mathcal{T}}$ is infinite. This holds for any finite $\mathcal{T} \subset \Delta(\mathfrak{X})$. Every element of $\mathfrak{E}$ must contain $\mathcal{I}_{\mathcal{T}}$ for some finite $\mathcal{T} \subset \Delta(\mathfrak{X})$; thus, every element of $\mathfrak{E}$ is infinite.

(F1) Let $\mathcal{E}, \mathcal{F} \in \mathfrak{E}$. Then there exist finite sets $\mathcal{S}, \mathcal{T} \subset \Delta(\mathfrak{X})$ such that $\mathcal{I}_{\mathcal{S}} \subseteq \mathcal{E}$ and $\mathcal{I}_{\mathcal{T}} \subseteq \mathcal{F}$. But then $\mathcal{S} \cup \mathcal{T}$ is also finite, and $\mathcal{I}_{\mathcal{S} \cup \mathcal{T}}=\mathcal{I}_{\mathcal{S}} \cap \mathcal{I}_{\mathcal{T}} \subseteq \mathcal{E} \cap \mathcal{F}$; thus, $\mathcal{E} \cap \mathcal{F} \in \mathfrak{E}$.

(F2) Suppose $\mathcal{E} \in \mathfrak{E}$ and $\mathcal{E} \subseteq \mathcal{D}$. Then there is some finite $\mathcal{T} \subset \Delta(\mathfrak{X})$ such that $\mathcal{I}_{\mathcal{T}} \subseteq \mathcal{E}$. But then $\mathcal{I}_{\mathcal{T}} \subseteq \mathcal{D}$; thus $\mathcal{D} \in \mathfrak{E}$ also.

$\diamond$ Claim 2

Now Claim 2 and the Ultrafilter Lemma yields a free ultrafilter $\mathfrak{F}$ with $\mathfrak{E} \subseteq \mathfrak{F}$. Let ${ }^{*} \mathbb{R}$ be the hyperreal field defined by $\mathfrak{F}$ (see Appendix A). We define $\phi:[-1,1] \longrightarrow{ }^{*} \mathbb{R}$ as follows. First, for all $(\omega, \mathfrak{Y}) \in \mathcal{I}$, let $\phi_{\omega, \mathfrak{Y}}: \mathcal{Q}_{\omega} \longrightarrow \mathbb{R}$ be the odd, strictly increasing function from part (a). Next, for any $r \in[-1,1]$, define the element $\phi(r) \in \mathbb{R}^{\mathcal{I}}$ by:

$$
\phi_{\omega, \mathfrak{Y}}(r):=\left\{\begin{array}{rll}
\phi_{\omega, \mathfrak{Y}}(r) & \text { if } & r \in \mathcal{Q}_{\omega} \\
0 & \text { if } & r \notin \mathcal{Q}_{\omega}
\end{array}\right.
$$

Then define $\phi(r) \in{ }^{*} \mathbb{R}$ to be the $(\underset{\mathfrak{F}}{\sim}$ )-equivalence class of $\phi(r)$.

Claim 3: $\quad \phi$ is odd and strictly increasing. 
Proof: Odd. Let $r \in[-1,1]$. Find some $\mathcal{X} \in \mathfrak{X}$ and $\mu \in \Delta(\mathcal{X})$ such that $\widetilde{\mu}_{k}=r$ for some $k \in \mathcal{K}$; then for all $(\omega, \mathfrak{Y}) \in \mathcal{I}_{\{(\mathcal{X}, \mu)\}}$ we have $r \in \mathcal{Q}_{\omega}$, and $\phi_{\omega, \mathfrak{Y}}(-r)=-\phi_{\omega, \mathfrak{Y}}(r)$, because $\phi_{\omega, \mathfrak{Y}}$ is odd by part (a). But $\mathcal{I}_{\{(\mathcal{X}, \mu)\}} \in \mathfrak{F}$, by definition of $\mathfrak{F}$. Thus, we obtain $\phi(-r)=-\phi(r)$.

Increasing. Let $q, r \in[-1,1]$, with $q<r$. Find some $\mathcal{X} \in \mathfrak{X}$ and $\mu \in \Delta(\mathcal{X})$ such that $\widetilde{\mu}_{k}=r$ and $\widetilde{\mu}_{j}=q$ for some $j, k \in \mathcal{K}$; then for all $\omega \in \Omega_{\{(\mathcal{X}, \mu)\}}$, we have $q, r \in \mathcal{Q}_{\omega}$, and $\phi_{\omega, \mathfrak{Y}}(q)<\phi_{\omega, \mathfrak{Y}}(r)$, because $\phi_{\omega, \mathfrak{Y}}$ is increasing by part (a). But $\mathcal{I}_{\{(\mathcal{X}, \mu)\}} \in \mathfrak{F}$ by definition of $\mathfrak{F}$. Thus, we obtain $\phi(q)<\phi(r)$.

$\diamond$ Claim 3

Now, let $\mathcal{X} \in \mathfrak{X}, \mu \in \Delta(\mathcal{X})$ and let $\mathrm{x} \in F(\mathcal{X}, \mu)$; we must show that $\mathrm{x} \in F_{\phi}(\mathcal{X}, \mu)$. For all $(\omega, \mathfrak{Y}) \in \mathcal{I}_{\{(\mathcal{X}, \mu)\}}$, part (a) says that $F(\mathcal{X}, \mu) \subseteq F_{\phi_{\omega}}(\mathcal{X}, \mu)$; thus, for all $\mathbf{y} \in \mathcal{X}$, we have $(\mathbf{x}-\mathbf{y}) \bullet \phi_{\omega, \mathfrak{Y}}(\mu) \geq 0$. But $\mathcal{I}_{\{(\mathcal{X}, \mu)\}} \in \mathfrak{F}$. Thus, $(\mathbf{x}-\mathbf{y}) \bullet \phi(\mu) \geq 0$. This holds for all $\mathbf{y} \in \mathcal{X}$; thus, $\mathbf{x} \in F_{\phi}(\mathcal{X}, \mu)$.

This holds for all $\mathrm{x} \in F(\mathcal{X}, \mu)$; thus, $F(\mathcal{X}, \mu) \subseteq F_{\phi}(\mathcal{X}, \mu)$.

Since $F(\mathcal{X}, \mu)$ must be nonempty, we must have $F(\mathcal{X}, \mu)=F_{\phi}(\mathcal{X}, \mu)$ whenever $F_{\phi}(\mathcal{X}, \mu)$ is single-valued. But $\phi$ is strictly increasing, so $F_{\phi}$ is single-valued on a dense, open subset of $\Delta(\mathcal{X})$, by Proposition 3.5(c).

(c) The rule $F_{\phi}$ is monotone, by Proposition 3.5. Meanwhile $F$ is UHC by hypothesis. Thus, Lemma C.1 implies that $F(\mu)=F_{\phi}(\mu)$ for all $\mu \in \Delta(\mathcal{X})$.

Proof of Theorem 3.1. Let $\mathfrak{X}:=\langle\mathcal{X}\rangle$. Fix $W \in \mathbb{N}$, and define $\omega(n):=1$ for all $n \in[1 \ldots W]$ while $\omega(0):=1$ for all $n>W$; then $\Delta_{\omega}\langle\mathcal{X}\rangle=\Delta_{W}\langle\mathcal{X}\rangle$. Now apply Theorem 3.4(a)

Proof of Theorem 3.3. Let $\mathfrak{X}:=\langle\mathcal{X}\rangle$, and apply Theorem 3.4(c)

Proof of Proposition 3.6. Fix $\mathcal{K}$ and $\mathcal{X} \subseteq\{ \pm 1\}^{\mathcal{K}}$. Let $\left\{\mu^{n}\right\}_{n=1}^{\infty} \subset \Delta(\mathcal{X})$ and $\mu \in \Delta(\mathcal{X})$, and suppose $\mu^{n} \underset{n \rightarrow \infty}{\longrightarrow} \mu$. Let $\mathbf{x} \in \mathcal{X}$, and suppose $\mathbf{x} \in F_{\phi}\left(\mu^{n}\right)$ for all sufficiently large $n \in \mathbb{N}$; we must show that $\mathbf{x} \in F_{\phi}(\mu)$. Let $\mathbf{y} \in \mathcal{X} \backslash\{\mathbf{x}\}$. Then $\left(\lim _{n \rightarrow \infty} \mu^{n}=\mu\right)$

$$
\begin{aligned}
& \Longrightarrow \quad\left(\lim _{n \rightarrow \infty} \widetilde{\mu}^{n}=\widetilde{\mu}\right) \underset{\overline{(*)}}{\Longrightarrow}\left(\lim _{n \rightarrow \infty} \phi\left(\widetilde{\mu}_{k}^{n}\right)=\phi\left(\widetilde{\mu}_{k}\right), \text { for all } k \in \mathcal{K}_{ \pm}(\mathbf{x}, \mathbf{y})\right) \\
& \Longrightarrow \quad\left(\lim _{n \rightarrow \infty} \sum_{k \in \mathcal{K}_{ \pm}(\mathbf{x}, \mathbf{y})}\left(x_{k}-y_{k}\right) \phi\left(\widetilde{\mu}_{k}^{n}\right)=\sum_{k \in \mathcal{K}_{ \pm}(\mathbf{x}, \mathbf{y})}\left(x_{k}-y_{k}\right) \phi(\widetilde{\mu})\right) \\
& \Longleftrightarrow\left(\lim _{n \rightarrow \infty}(\mathbf{x}-\mathbf{y}) \bullet \phi\left(\widetilde{\mu}^{n}\right)=(\mathbf{x}-\mathbf{y}) \bullet \phi(\widetilde{\mu})\right) \\
& \Longrightarrow((\mathbf{x}-\mathbf{y}) \bullet \phi(\widetilde{\mu}) \geq 0) .
\end{aligned}
$$

Here, $(*)$ is because the map $\Delta(\mathcal{X}) \ni \mu \mapsto \widetilde{\mu} \in \mathbb{R}^{\mathcal{K}}$ is continuous, while $(\dagger)$ is because $\phi$ is continuous at $\widetilde{\mu}_{k}$ for all $k \in \mathcal{K}_{ \pm}(\mathbf{x}, \mathbf{y})$, by hypothesis. Finally $(\ddagger)$ is because $(\mathbf{x}-\mathbf{y}) \bullet$ $\phi\left(\widetilde{\mu}^{n}\right) \geq 0$ for all large enough $n \in \mathbb{N}$, because $\mathbf{x} \in F_{\phi}\left(\mu^{n}\right)$ by hypothesis.

Thus, $\mathbf{x} \bullet \phi(\widetilde{\mu}) \geq \mathbf{y} \bullet \phi(\widetilde{\mu})$ for all $\mathbf{y} \in \mathcal{X}$. Thus, $\mathbf{x} \in F_{\phi}(\mu)$ as desired. 


\section{Proofs from $\S 6$}

The proof of Theorem 4.1(b) depends on results from $\S 5$, which in turn depend on results from $\S 6$. Thus, we will first prove the results from $\S 6$, then $\S 5$, and finally, $\S 4$. We need some preliminary lemmas before we can prove the results in from $\S 6$. Let $\phi:[-1,1] \longrightarrow{ }^{*}$ be odd and increasing. Let $\mathcal{C}:=\operatorname{conv}(\mathcal{X})$. For any $\mathrm{x} \in \mathcal{X}$, recall that $\mathcal{C}_{\mathrm{x}}^{\phi}:=\left\{\mathbf{c} \in \mathcal{C} ; \mathrm{x} \in F_{\phi}(\mathbf{c})\right\}$. For any $\mathbf{x}, \mathbf{y} \in \mathcal{X}$, recall that ${ }^{\circ} \mathcal{B}_{\mathbf{x}, \mathbf{y}}^{\phi}:=\{\mathbf{c} \in \operatorname{int}(\mathcal{C}) ; F(\mathbf{c})=\{\mathbf{x}, \mathbf{y}\}\}$. Parts $(\mathrm{c}, \mathrm{d}, \mathrm{e})$ of the next result are used in the proofs of Theorems E.7(b) and 4.1(b). Part (f) is necessary to derive Theorem 6.4 from Theorem E.7.

Lemma E.4 Let $\phi:[-1,1] \longrightarrow{ }^{*} \mathbb{R}$ be odd and increasing. Let $\mathcal{X}$ be a judgement space, and suppose $F_{\phi}: \Delta(\mathcal{X}) \rightrightarrows \mathcal{X}$ is upper hemicontinuous. Let $\mathbf{x}, \mathbf{y} \in \mathcal{X}$, and let $\mathbf{b} \in \mathcal{B}_{\mathbf{x}, \mathbf{y}}^{\phi}$.

(a) Let $k \in \mathcal{K}_{ \pm}(\mathbf{x}, \mathbf{y})$. For any $\epsilon>0$, if $\mathbf{b}+\epsilon x_{k} \mathbf{e}_{k} \in \mathcal{C}$, then $\mathbf{b}+\epsilon x_{k} \mathbf{e}_{k} \in \mathcal{C} \backslash \mathcal{C}_{\mathbf{y}}^{\phi}$.

Now suppose $\mathcal{X}$ is thick and $\mathbf{b} \in{ }^{\circ} \mathcal{B}_{\mathbf{x}, \mathbf{y}}^{\phi}$.

(b) Let $k \in \mathcal{K}_{ \pm}(\mathbf{x}, \mathbf{y})$. If $\epsilon$ is small enough, then $\mathbf{b}+\epsilon x_{k} \mathbf{e}_{k} \in \mathcal{C}_{\mathbf{x}}^{\phi} \backslash \mathcal{C}_{\mathbf{y}}^{\phi}$.

(c) Let $i, j \in \mathcal{K}_{ \pm}(\mathbf{x}, \mathbf{y})$. There exists $\epsilon_{0}>0$ and a continuous, increasing function $\delta_{i j}:\left(-\epsilon_{0}, \epsilon_{0}\right) \longrightarrow \mathbb{R}$ such that, for all $\epsilon \in\left(-\epsilon_{0}, \epsilon_{0}\right)$, we have $\mathbf{b}+\epsilon x_{i} \mathbf{e}_{i}+\delta_{i j}(\epsilon) y_{j} \mathbf{e}_{j} \in$ ${ }^{o} \mathcal{B}_{\mathbf{x}, \mathbf{y}}^{\phi} \cdot$

(d) $\mathcal{R}_{\mathcal{X}}^{\phi}$ is always an open set.

(e) Every $F_{\phi}$-pivotal class is an open subset of $[-1,1]$, and a union of connected components of $\mathcal{R}_{\mathcal{X}}^{\phi}$.

(f) Thus, if $\mathcal{R}_{\mathcal{X}}^{\phi}$ is connected, then $\mathcal{X}$ has only one $F_{\phi}$-pivotal class.

Proof: (a) Find profiles $\mu, \nu \in \Delta(\mathcal{X})$, such that $\widetilde{\mu}=\mathbf{b}$ and $\widetilde{\nu}=\mathbf{b}+\epsilon x_{k} \mathbf{e}_{k}$. By hypothesis, $\mathbf{x}, \mathbf{y} \in F_{\phi}(\mu)$, so $\mathbf{x} \bullet \phi(\widetilde{\mu})=\mathbf{y} \bullet \phi(\widetilde{\mu})$. Let $\delta:=\phi\left(b_{k}+\epsilon\right)-\phi\left(b_{k}\right)$; then $\delta>0$ (because $\phi$ is increasing), and we have

$$
\begin{aligned}
\mathbf{x} \bullet \phi(\widetilde{\nu}) & =\mathbf{x} \bullet \phi(\widetilde{\mu})+\delta ; \\
\text { and } \mathbf{y} \bullet \phi(\widetilde{\nu}) & =\mathbf{y} \bullet \phi(\widetilde{\mu})-\delta<\mathbf{x} \bullet \phi(\widetilde{\nu}) .
\end{aligned}
$$

Thus, $\mathbf{y} \notin F_{\phi}(\nu)$. Thus, $\mathbf{b}+\epsilon x_{k} \mathbf{e}_{k} \in \mathcal{C} \backslash \mathcal{C}_{\mathbf{y}}^{\phi}$.

(b) If $\mathbf{b} \in{ }^{\circ} \mathcal{B}_{\mathbf{x}, \mathbf{y}}^{\phi}$, then $F_{\phi}(\mathbf{b})=\{\mathbf{x}, \mathbf{y}\}$. Thus, if $\epsilon$ is small enough, then $F_{\phi}\left(\mathbf{b}+\epsilon x_{k} \mathbf{e}_{k}\right) \subseteq\{\mathbf{x}, \mathbf{y}\}$, because $F_{\phi}$ is upper hemicontinuous. Thus, we must have $\mathbf{b}+\epsilon x_{k} \mathbf{e}_{k} \in \mathcal{C}_{\mathbf{x}}^{\phi} \backslash \mathcal{C}_{\mathbf{y}}^{\phi}$.

(c) (by contradiction) First we consider the case when $\epsilon>0$.

There exists some $\epsilon_{1}>0$ such that $\mathbf{c} \in \operatorname{int}(\mathcal{C})$ for all $\mathbf{c} \in \mathbb{R}^{\mathcal{K}}$ with $\|\mathbf{c}-\mathbf{b}\|_{\infty}<\epsilon_{1}$ (because $\mathbf{b} \in \operatorname{int}(\mathcal{C})$ by definition of $\left.{ }^{o} \mathcal{B}_{\mathbf{x}, \mathbf{y}}^{\phi}\right)$. Next, there exists some $\epsilon_{2} \in\left(0, \epsilon_{1}\right)$ such that $F_{\phi}(\mathbf{c}) \subseteq\{\mathbf{x}, \mathbf{y}\}$ for all $\mathbf{c} \in \mathcal{C}$ with $\|\mathbf{c}-\mathbf{b}\|_{\infty}<\epsilon_{2}$-otherwise, we have a contradiction of upper hemicontinuity. 
For all $\epsilon \in\left(0, \epsilon_{2}\right)$, part (b) says $\mathbf{b}+\epsilon x_{i} \mathbf{e}_{i} \in \mathcal{C}_{\mathbf{x}}^{\phi} \backslash \mathcal{C}_{\mathbf{y}}^{\phi}$. We claim there exists $\epsilon_{0}>0$ such that, for all $\epsilon \in\left(0, \epsilon_{0}\right)$, there exists $\delta_{i j}(\epsilon) \in\left(0, \epsilon_{2}\right)$ such that $\mathbf{b}+\epsilon x_{i} \mathbf{e}_{i}+\delta_{i j}(\epsilon) y_{j} \mathbf{e}_{j} \epsilon$ ${ }^{\circ} \mathcal{B}_{\mathbf{x}, \mathbf{y}}^{\phi}$. Suppose not. Then for any $\epsilon_{0} \in\left(0, \epsilon_{2}\right)$, there exists some $\epsilon_{1} \in\left(0, \epsilon_{0}\right)$ such that $\mathbf{b}+\epsilon_{1} x_{i} \mathbf{e}_{i}+\delta y_{j} \mathbf{e}_{j} \in \mathcal{C}_{\mathbf{x}}^{\phi} \backslash \mathcal{C}_{\mathbf{y}}^{\phi}$ for all $\delta \in\left(0, \epsilon_{2}\right)$.

Claim 1: For any $\epsilon \in\left(\epsilon_{1}, \epsilon_{2}\right)$, we have $\mathbf{b}+\epsilon x_{i} \mathbf{e}_{i}+\delta y_{j} \mathbf{e}_{j} \in \mathcal{C}_{\mathbf{x}}^{\phi} \backslash \mathcal{C}_{\mathbf{y}}^{\phi}$ for all $\delta \in\left(0, \epsilon_{2}\right)$.

Proof: (by contradiction) For any such $\epsilon$ and $\delta$, we have $\mathbf{b}+\epsilon x_{i} \mathbf{e}_{i}+\delta y_{j} \mathbf{e}_{j} \in \mathcal{C}_{\mathbf{x}}^{\phi} \cup \mathcal{C}_{\mathbf{y}}^{\phi}$ by construction of $\epsilon_{2}$. Suppose $\mathbf{b}+\epsilon x_{i} \mathbf{e}_{i}+\delta y_{j} \mathbf{e}_{j} \in \mathcal{C}_{\mathbf{y}}^{\phi}$ for some $\epsilon \in\left(\epsilon_{1}, \epsilon_{2}\right)$ and $\delta \in\left(0, \epsilon_{2}\right)$. Fix $\epsilon$, and let $\delta^{\prime} \in\left(0, \epsilon_{2}\right)$ be the infimum of all $\delta \in\left(0, \epsilon_{2}\right)$ such that $\mathbf{b}+\epsilon x_{i} \mathbf{e}_{i}+\delta y_{j} \mathbf{e}_{j} \in$ $\mathcal{C}_{\mathbf{y}}^{\phi}$. Then $\mathbf{b}+\epsilon x_{i} \mathbf{e}_{i}+\delta^{\prime} y_{j} \mathbf{e}_{j} \in \mathcal{C}_{\mathbf{y}}^{\phi}$, because $F_{\phi}$ is upper hemicontinuous. Meanwhile, $\mathbf{b}+\epsilon x_{i} \mathbf{e}_{i}+\delta y_{j} \mathbf{e}_{j} \in \mathcal{C}_{\mathbf{x}}^{\phi}$ for all $\delta \in\left(0, \delta^{\prime}\right)$. Thus, $\mathbf{b}+\epsilon x_{i} \mathbf{e}_{i}+\delta^{\prime} y_{j} \mathbf{e}_{j} \in \mathcal{C}_{\mathbf{x}}^{\phi}$, again by upper hemicontinuity. Thus, $\mathbf{b}+\epsilon x_{i} \mathbf{e}_{i}+\delta^{\prime} y_{j} \mathbf{e}_{j} \in{ }^{\circ} \mathcal{B}_{\mathbf{x}, \mathbf{y}}^{\phi}$.

Thus, part (b) says that $\mathbf{b}+\epsilon^{\prime} x_{i} \mathbf{e}_{i}+\delta^{\prime} y_{j} \mathbf{e}_{j} \in \mathcal{C}_{\mathbf{y}}^{\phi} \backslash \mathcal{C}_{\mathbf{x}}^{\phi}$ for all $\epsilon^{\prime} \in(0, \epsilon)$. In particular, this means that $\mathbf{b}+\epsilon_{1} x_{i} \mathbf{e}_{i}+\delta^{\prime} y_{j} \mathbf{e}_{j} \in \mathcal{C}_{\mathbf{y}}^{\phi} \backslash \mathcal{C}_{\mathbf{x}}^{\phi}$. But this contradicts the hypothesis defining $\epsilon_{1}$.

$\diamond$ Claim 1

Since we can make $\epsilon_{1}$ arbitrarily small, Claim 1 means that $\mathbf{b}+\epsilon x_{i} \mathbf{e}_{i}+\delta y_{j} \mathbf{e}_{j} \in \mathcal{C}_{\mathbf{x}}^{\phi} \backslash \mathcal{C}_{\mathbf{y}}^{\phi}$ for all $\epsilon, \delta \in\left(0, \epsilon_{2}\right)$. Now fix $\delta \in\left(0, \epsilon_{2}\right)$ and let $\epsilon$ go to zero; then upper hemicontinuity implies that $\mathbf{b}+\delta y_{j} \mathbf{e}_{j} \in \mathcal{C}_{\mathbf{x}}^{\phi}$. But for all $\delta>0$, part (b) says $\mathbf{b}+\delta y_{j} \mathbf{e}_{j} \in \mathcal{C}_{\mathbf{y}}^{\phi} \backslash \mathcal{C}_{\mathbf{x}}^{\phi}$. Contradiction.

The proof for $\epsilon<0$ is similar, with the roles of $\mathbf{x}$ and $\mathbf{y}$ reversed.

A similar construction yields a function $\delta_{j i}:\left(-\epsilon_{1}, \epsilon_{1}\right) \longrightarrow \mathbb{R}$ such that, for all $\epsilon \in\left(-\epsilon_{1}, \epsilon_{1}\right)$, we have $\mathbf{b}+\epsilon y_{j} \mathbf{e}_{j}+\delta_{j i}(\epsilon) x_{i} \mathbf{e}_{i} \in{ }^{o} \mathcal{B}_{\mathbf{x}, \mathbf{y}}^{\phi}$. Thus, $\delta_{j i}=\delta_{i j}^{-1}$. Thus, both $\delta_{i j}$ and $\delta_{j i}$ are invertible. Clearly they are nondecreasing. It follows that they are continuous and strictly increasing.

(d) If $\mathcal{R}_{\mathcal{X}}^{\phi}=\emptyset$, then it is automatically open. So, suppose $\mathcal{R}_{\mathcal{X}}^{\phi} \neq \emptyset$, and let $r \in \mathcal{R}_{\mathcal{X}}^{\phi}$. Then there exists $\mathbf{x}, \mathbf{y} \in \mathcal{X}$ with $d(\mathbf{x}, \mathbf{y}) \geq 3$, and $\mathbf{b} \in{ }^{o} \mathcal{B}_{\mathbf{x}, \mathbf{y}}^{\phi}$, and $i \in \mathcal{K}_{ \pm}(\mathbf{x}, \mathbf{y})$ such that $r=b_{i}$. But then part (c) yields some $\epsilon_{0}>0$ such that, for all $r^{\prime} \in\left(r-\epsilon_{0}, r+\epsilon_{0}\right)$, there is some $\mathbf{b}^{\prime} \in{ }^{o} \mathcal{B}_{\mathbf{x}, \mathbf{y}}^{\phi}$ with $\mathbf{b}_{i}^{\prime}=r^{\prime}$. Thus, $\left(r-\epsilon_{0}, r+\epsilon_{0}\right) \subseteq \mathcal{R}_{\mathcal{X}}^{\phi}$; thus, $\mathcal{R}_{\mathcal{X}}^{\phi}$ is an open set.

(e) Let $r \in \mathcal{R}_{\mathcal{X}}^{F}$.

Claim 2: $\quad$ The set $\mathcal{E}^{1}(r):=\left\{q \in \mathcal{R}_{\mathcal{X}}^{\phi} ; r \sim q\right\}$ is open.

Proof: If $q \in \mathcal{E}^{1}(r)$, then there exist $\mathbf{x}, \mathbf{y} \in \mathcal{X}$ with $d(\mathbf{x}, \mathbf{y}) \geq 3$, and $\mathbf{b} \in{ }^{o} \mathcal{B}_{\mathbf{x}, \mathbf{y}}^{F}$ and $j, k \in \mathcal{K}_{ \pm}(\mathbf{x}, \mathbf{y})$, such that $b_{k}=r$ and $b_{j}=q$. Since $d(\mathbf{x}, \mathbf{y}) \geq 3$, there is some $i \in \mathcal{K}_{ \pm}(\mathbf{x}, \mathbf{y}) \backslash\{j, k\}$. Let $\delta_{i j}:\left(-\epsilon_{0}, \epsilon_{0}\right) \longrightarrow \mathbb{R}$ be as in part (c). Then for all $\epsilon \in\left(-\epsilon_{0}, \epsilon_{0}\right)$, we have $r \sim q+\delta_{i j}(\epsilon)$.

$\diamond$ Claim 2

Inductively, for all $n \in \mathbb{N}$, define $\mathcal{E}^{n}(r):=\bigcup_{q \in \mathcal{E}^{n-1}(r)} \mathcal{E}^{1}(q)$. Then Claim 2 implies that $\mathcal{E}^{n}(r)$ contains a neighbourhood of each element of $\mathcal{E}^{n-1}(r)$. Finally, let $\mathcal{E}^{\infty}(r):=$ $\bigcup_{n=1}^{\infty} \mathcal{E}^{n}(r)$. Then $\mathcal{E}^{\infty}(r)$ is open, and is the pivotal class of $r$.

Claim 3: $\quad$ Let $\mathcal{R}^{\prime}$ be a connected component of $\mathcal{R}_{\mathcal{X}}^{F}$. Then all elements of $\mathcal{R}^{\prime}$ are $(\approx)$-equivalent. 
Proof: Each $(\approx)$-equivalence classes is an open subset of $\mathbb{R}$, so its intersection with $\mathcal{R}^{\prime}$ is a relatively open subset of $\mathcal{R}^{\prime}$. Thus, the $(\approx)$-equivalence classes form an open partition of $\mathcal{R}^{\prime}$. But $\mathcal{R}^{\prime}$ is connected, so this partition must contain only one element. In other words, all elements of $\mathcal{R}^{\prime}$ are $(\approx)$-equivalent.

$\diamond$ Claim 3

It follows that each $(\approx)$-equivalence class is a union of connected components of $\mathcal{R}_{\mathcal{X}}^{F}$.

(f) follows immediately from part (e): if $\mathcal{R}_{\mathcal{X}}^{F}$ has only one connected component, then it can have only one pivotal class.

Let $\phi:[-1,1] \longrightarrow{ }^{*} \mathbb{R}$ be a gain function. For all $r \in[-1,1]$, define $\mathbb{G}(\phi, r):=\left\{{ }^{*} r ;{ }^{*} r \in{ }^{*} \mathbb{R}\right.$ and $\left|{ }^{*} r\right|<\phi\left(r+\epsilon_{1}\right)-\phi\left(r-\epsilon_{2}\right)$ for all $\left.\epsilon_{1}, \epsilon_{2}>0\right\}$. Observe that, for any hyperreal scalar ${ }^{*} s>0$, the function st $\left({ }^{*} s \phi\right)$ is continuous at $r$ if and only if $\mathbb{G}\left({ }^{*} s \phi, r\right)$ contains only infinitesimal elements of ${ }^{*} \mathbb{R}$. Otherwise, $\mathbb{G}\left({ }^{*} s \phi, r\right)$ measures the size of the 'gap' at $r$. The proof of Proposition 6.2(a) uses the following lemma.

Lemma E.5 Let $\mathcal{X}$ be a thick judgement space, let $\phi:[-1,1] \longrightarrow{ }^{*} \mathbb{R}$ be a gain function, and suppose $F_{\phi}$ is upper hemicontinuous on $\Delta(\mathcal{X})$. If $r \sim s \in \mathcal{R}_{\mathcal{X}}^{F}$, then $\mathbb{G}(\phi, r)=\mathbb{G}(\phi, s)$.

Proof: We will show that $\mathbb{G}(\phi, s) \subseteq \mathbb{G}(\phi, r)$.

If $s \sim r$, then there exist $\mathbf{x}, \mathbf{y} \in \mathcal{X}$, with $d(\mathbf{x}, \mathbf{y}) \geq 3$, and $\mathbf{b} \in{ }^{o} \mathcal{B}_{\mathbf{x}, \mathbf{y}}^{\phi}$, and $i, j \in \mathcal{K}_{ \pm}(\mathbf{x}, \mathbf{y})$ such that $b_{i}=r$ and $b_{j}=s$. Let

$$
C:=\sum_{k \in \mathcal{K}_{ \pm}(\mathbf{x}, \mathbf{y}) \backslash\{i, j\}}\left(x_{k}-y_{k}\right) \phi\left(b_{k}\right) .
$$

Assume without loss of generality that $x_{i}=x_{j}=1$ and $y_{i}=y_{j}=-1$. If $\mathbf{b} \in{ }^{o} \mathcal{B}_{\mathbf{x}, \mathbf{y}}^{\phi}$, then $2 \phi\left(b_{i}\right)+2 \phi\left(b_{j}\right)+C=(\mathbf{x}-\mathbf{y}) \bullet \phi(\mathbf{b})=0$.

Lemma E.4(c) yields $\epsilon_{0}>0$ and a continuously increasing function $\delta_{i j}:\left(-\epsilon_{0}, \epsilon_{0}\right) \longrightarrow \mathbb{R}$ such that, for all $\epsilon \in\left(-\epsilon_{0}, \epsilon_{0}\right)$, we have $\mathbf{b}+\epsilon x_{i} \mathbf{e}_{i}+\delta_{i j}(\epsilon) y_{j} \mathbf{e}_{j} \in{ }^{o} \mathcal{B}_{\mathbf{x}, \mathbf{y}}^{\phi}$. Thus, for all $\epsilon \in\left(-\epsilon_{0}, \epsilon_{0}\right)$, we have

$$
2 \phi\left(b_{i}+\epsilon\right)+2 \phi\left(b_{j}-\delta_{i j}(\epsilon)\right)+C=0 .
$$

Thus, for any $\epsilon_{1}, \epsilon_{2}>0$, we have

$$
\begin{aligned}
0 & =0-0=\left(2 \phi\left(b_{i}+\epsilon_{1}\right)+2 \phi\left(b_{j}-\delta_{i j}\left(\epsilon_{1}\right)\right)+C\right)-\left(2 \phi\left(b_{i}-\epsilon_{2}\right)+2 \phi\left(b_{j}-\delta_{i j}\left(-\epsilon_{2}\right)\right)+C\right) \\
& =2\left(\phi\left(b_{i}+\epsilon_{1}\right)-\phi\left(b_{i}-\epsilon_{2}\right)\right)+2\left(\phi\left(b_{j}-\delta_{i j}\left(\epsilon_{1}\right)\right)-\phi\left(b_{j}-\delta_{i j}\left(-\epsilon_{2}\right)\right)\right) \\
& =2\left(\phi\left(r+\epsilon_{1}\right)-\phi\left(r-\epsilon_{2}\right)\right)+2\left(\phi\left(s-\delta_{i j}\left(\epsilon_{1}\right)\right)-\phi\left(s-\delta_{i j}\left(-\epsilon_{2}\right)\right)\right) .
\end{aligned}
$$

Thus,

$$
\phi\left(r+\epsilon_{1}\right)-\phi\left(r-\epsilon_{2}\right)=\phi\left(s-\delta_{i j}\left(-\epsilon_{2}\right)\right)-\phi\left(s-\delta_{i j}\left(\epsilon_{1}\right)\right) .
$$

Now, let $g \in \mathbb{G}(\phi, s)$. Then $|g| \leq \phi\left(s+\delta_{2}\right)-\phi\left(s+\delta_{1}\right)$ for all $\delta_{1}, \delta_{2}>0$. Thus, $|g| \leq \phi\left(s-\delta_{i j}\left(-\epsilon_{2}\right)\right)-\phi\left(s-\delta_{i j}\left(\epsilon_{1}\right)\right)$ for all $\epsilon_{1}, \epsilon_{2}>0$. (Note that $-\delta_{i j}\left(-\epsilon_{2}\right)>0$, because 
$\delta_{i j}$ is increasing and $\delta_{i j}(0)=0$.) Thus, equation (E8) says that $|g| \leq \phi\left(r+\epsilon_{1}\right)-\phi\left(r-\epsilon_{2}\right)$ for all $\epsilon_{1}, \epsilon_{2}>0$. Thus, $g \in \mathbb{G}(\phi, r)$, as desired. Thus $\mathbb{G}(\phi, s) \subseteq \mathbb{G}(\phi, r)$.

An identical argument shows $\mathbb{G}(\phi, r) \subseteq \mathbb{G}(\phi, s)$. We conclude $\mathbb{G}(\phi, r)=\mathbb{G}(\phi, s)$.

Proof of Lemma 6.1. (a) Nonempty. (by contradiction) Suppose $\mathcal{R}_{\mathcal{X}}^{\phi}=\emptyset$.

Claim 1: For any continuous, increasing gain function $\psi:[-1,1] \longrightarrow \mathbb{R}$, we have $F_{\phi}=F_{\psi}$.

Proof: $F_{\psi}$ is upper hemicontinuous by Proposition 3.6, while $F_{\phi}$ is upper hemicontinuous by hypothesis. Meanwhile, Proposition $3.5(\mathrm{a}, \mathrm{b})$ say that both $F_{\phi}$ and $F_{\psi}$ are SME and monotone.

If $\mathcal{R}_{\mathcal{X}}^{\phi}=\emptyset$, then for any $\mathbf{x}, \mathbf{y} \in \mathcal{X}$ with $d_{H}(\mathbf{x}, \mathbf{y}) \geq 3$, we must have ${ }^{o} \mathcal{B}_{\mathbf{x}, \mathbf{y}}^{\phi}=\emptyset$. Thus, the right-hand condition in Proposition C.2 is trivially satisfied. Thus, $F_{\psi}=F_{\phi}$. $\diamond$ Claim 1

In particular, let $\Phi_{I}$ be the set of all odd, continuous, real-valued gain functions. Then we have

$$
\operatorname{SSME}(\mathcal{X}, \mu) \overline{\overline{(*)}} \bigcup_{\psi \in \Phi_{I}} F_{\psi}(\mu) \overline{\overline{(\dagger)}} F_{\phi}(\mu)
$$

where $(*)$ is by Proposition 1.1(b), and $(\dagger)$ is by Claim 1.

Thus, $\mathcal{X}$ is supermajoritarian determinate. Contradiction.

Open follows from Lemma E.4(d).

Proof of Theorem 6.2. (a) For all $r \in[-1,1]$, define $\hat{\phi}(r):=\frac{\phi(r)-\phi\left(r_{1}\right)}{\phi\left(r_{2}\right)-\phi\left(r_{1}\right)}$, to obtain a function $\hat{\phi}:[-1,1] \longrightarrow \mathbb{R}$. Define $\mathbb{G}(\hat{\phi}, r)$ as in Lemma E.5. Let $\mathbb{I}$ be the set of all infinitesimal elements of *R.

Claim 1: For any $r \in \mathcal{R}$, we have $\mathbb{G}(\hat{\phi}, r) \subseteq \mathbb{I}$.

Proof: (by contradiction) Suppose there exists some $r \in \mathcal{R}$ with some $g \in \mathbb{G}(\hat{\phi}, r)$ which is not infinitesimal. Since $\mathbb{G}(\hat{\phi}, r)$ is symmetric under negation, we can assume $g>0$ without loss of generality.

Let $\mathcal{R}^{\prime}:=\left(r_{1}, r_{2}\right) \cap \mathcal{R}$ (an open subset of $\mathcal{R}$ ). For any $r^{\prime} \in \mathcal{R}^{\prime}$, we have $0=\hat{\phi}\left(r_{1}\right)<$ $\hat{\phi}\left(r^{\prime}\right)<\hat{\phi}\left(r_{2}\right)=1$; thus, $\hat{\phi}\left(r^{\prime}\right)$ is finite. Furthermore, $\mathcal{R}$ is connected, so Lemma E.4(e) says that $r \sim r^{\prime}$ for all $r^{\prime} \in \mathcal{R}$. Thus, Lemma E.5 says that $g \in \mathbb{G}\left(\hat{\phi}, r^{\prime}\right)$ for all $r^{\prime} \in \mathcal{R}^{\prime}$. That is: for all $s_{1}, s_{2} \in \mathcal{R}$, if $s_{1}<r^{\prime}<s_{2}$, then $\hat{\phi}\left(s_{2}\right)-\hat{\phi}\left(s_{1}\right)>g$. Since $g \notin \mathbb{I}$ and $\hat{\phi}\left(r^{\prime}\right) \in[0,1]$, this implies there is rational number $q_{r^{\prime}} \in \mathbb{Q} \cap[0,1]$ such that for all $s_{1}, s_{2} \in \mathcal{R}$, if $s_{1}<r^{\prime}<s_{2}$, then $\hat{\phi}\left(s_{1}\right)<q_{r^{\prime}}<\hat{\phi}\left(s_{2}\right)$.

Furthermore, if $r^{\prime}<r^{\prime \prime} \in \mathcal{R}^{\prime}$, then $q_{r^{\prime}}<q_{r^{\prime \prime}}$ (because for any $s \in\left(r^{\prime}, r^{\prime \prime}\right)$, we have $\left.q_{r^{\prime}}<\hat{\phi}(s)<q_{r^{\prime \prime}}\right)$. Thus, the mapping $\mathcal{R}^{\prime} \ni r \mapsto q_{r} \in \mathbb{Q} \cap[0,1]$ is injective. But $\mathcal{R}^{\prime}$ is uncountable (it is an open subset of $\mathbb{R}$ ), whereas $\mathbb{Q}$ is countable. This is a contradiction.

$\diamond$ Claim 1 
Claim 2: $\quad \bar{\phi}$ is continuous on $\mathcal{R}$.

Proof: Let $r \in \mathcal{R}$. If $\bar{\phi}$ is discontinuous at $r$, then $\mathbb{G}(\hat{\phi}, r)$ contains a non-infinitesimal value, contradicting Claim 1.

$\diamond$ Claim 2

Claim 3: $\quad$ For any $r \in \mathcal{R}$, we have $\mathbb{G}(\hat{\phi}, r) \supseteq \mathbb{I}$.

Proof: (by contradiction) Suppose there exists some $r^{\prime} \in \mathcal{R}$ with some $i \in \mathbb{I}$ such that $i \notin \mathbb{G}\left(\hat{\phi}, r^{\prime}\right)$. Since $\mathbb{G}\left(\hat{\phi}, r^{\prime}\right)$ and $\mathbb{I}$ are symmetric under negation, we can assume $i>0$ without loss of generality. Then for all $r \in \mathcal{R}$, Lemma E.5 says that $i \notin \mathbb{G}(\hat{\phi}, r)$. Thus, there exists some $\epsilon_{r}>0$ such that $\hat{\phi}\left(r+\epsilon_{r}\right)-\hat{\phi}\left(r-\epsilon_{r}\right)<i$, and hence, $|\hat{\phi}(s)-\hat{\phi}(r)|<i$ for all $s \in \mathcal{R} \cap\left(r-\epsilon_{r}, r+\epsilon_{r}\right)$. Thus, $\bar{\phi}(s)=\bar{\phi}(r)$ for all $s \in \mathcal{R} \cap\left(r-\epsilon_{r}, r+\epsilon_{r}\right)$.

Thus, if we fix some $r_{0} \in \mathcal{R}$, and define $c_{0}:=\bar{\phi}(r)$, then the set $\mathcal{R}^{\prime}:=\{r \in \mathcal{R}$; $\left.\bar{\phi}(r)=c_{0}\right\}$ is an open neighbourhood around $r_{0}$ (because for all $r^{\prime} \in \mathcal{R}^{\prime}$, we have $\left.\left(r^{\prime}-\epsilon_{r^{\prime}}, r^{\prime}+\epsilon_{r^{\prime}}\right) \subseteq \mathcal{R}^{\prime}\right)$. But $\mathcal{R}^{\prime}$ is also closed in $\mathcal{R}$, because $\bar{\phi}$ is continuous on $\mathcal{R}$, by Claim 2. But $\mathcal{R}$ is connected, so this implies that $\mathcal{R}^{\prime}=\mathcal{R}$. Thus, $\bar{\phi}$ is constant on $\mathcal{R}$. But by definition, $\bar{\phi}\left(r_{2}\right)=1$ while $\bar{\phi}\left(r_{1}\right)=0$. Contradiction.

$\diamond$ Claim 3

It remains to show that $\bar{\phi}$ is increasing on $\mathcal{R}$. Suppose not. Then there is some $r \in \mathcal{R}$ and $\epsilon>0$ such that $\bar{\phi}$ is constant on $(r-\epsilon, r+\epsilon)$. But then there is some positive $i \in \mathbb{I}$ such that $|\hat{\phi}(s)-\hat{\phi}(r)|<i$ for all $s \in(r-\epsilon, r+\epsilon)$, which means $i \notin \mathbb{G}(\hat{\phi}, r)$, contradicting Claim 3.

(b) Let $\psi:[-1,1] \longrightarrow \mathbb{R}$ be a continuous gain function such that $\psi(r)=$ st $(s \phi(r))$ for all $r \in \mathrm{cl}\left(\mathcal{R}_{\mathcal{X}}^{F}\right)$. (Such a continuous extension exists exists by the Tietze Extension Theorem, because st $(s \phi)$ is continuous on $\operatorname{cl}\left(\mathcal{R}_{\mathcal{X}}^{F}\right)$ and $\operatorname{cl}\left(\mathcal{R}_{\mathcal{X}}^{F}\right)$ is a closed subset of $[-1,1]$.$) We will show that F_{\psi}=F_{\phi}$.

$F_{\phi}$ is upper hemicontinuous by hypothesis, and Proposition 3.6 says that $F_{\psi}$ is upper hemicontinuous, because $\psi$ is continuous on $[-1,1]$. Meanwhile, Proposition 3.5 says that both $F_{\psi}$ and $F_{\phi}$ are monotone and SME. Thus, we can apply Proposition C.2.

Claim 4: For any $\mathbf{x}, \mathbf{y} \in \mathcal{X}$, if $d_{H}(\mathbf{x}, \mathbf{y}) \geq 3$, then ${ }^{\circ} \mathcal{B}_{\mathbf{x}, \mathbf{y}}^{\phi} \cap \mathcal{C}_{\mathbf{x}}^{\psi} \subseteq \mathcal{C}_{\mathbf{y}}^{\psi}$.

Proof: Let $\mathbf{b} \in{ }^{o} \mathcal{B}_{\mathbf{x}, \mathbf{y}}^{\phi} \cap \mathcal{C}_{\mathbf{x}}^{\psi}$. Then

$$
\begin{aligned}
& \psi(\mathbf{b}) \bullet(\mathbf{x}-\mathbf{y})=\sum_{k \in \mathcal{K}_{ \pm}(\mathbf{x}, \mathbf{y})}\left(x_{k}-y_{k}\right) \cdot \psi\left(b_{k}\right) \overline{\overline{(*)}} \sum_{k \in \mathcal{K}_{ \pm}(\mathbf{x}, \mathbf{y})}\left(x_{k}-y_{k}\right) \cdot \operatorname{st}\left(s \phi\left(b_{k}\right)\right) \\
& \overline{\overline{(\diamond)}} \operatorname{st}\left(s \sum_{k \in \mathcal{K}_{ \pm}(\mathbf{x}, \mathbf{y})}\left(x_{k}-y_{k}\right) \cdot \phi\left(b_{k}\right)\right)=\operatorname{st}(s \phi(\mathbf{b}) \bullet(\mathbf{x}-\mathbf{y})) \overline{\overline{(\dagger)}} \operatorname{st}(0)=0 .
\end{aligned}
$$

Here, $(*)$ is because, for all $k \in \mathcal{K}_{ \pm}(\mathbf{x}, \mathbf{y})$, we have $b_{k} \in \mathcal{R}_{\mathcal{X}}^{F}$, and thus, $\psi\left(b_{k}\right)=$ st $\left(s \phi\left(b_{k}\right)\right)$. Next, $(\diamond)$ is because the function st : ${ }^{*} \mathbb{R} \longrightarrow \mathbb{R}$ is a ring homomorphism, so it preserves multiplication and addition. Finally, $(\dagger)$ is because $\mathbf{b} \in{ }^{o} \mathcal{B}_{\mathbf{x}, \mathbf{y}}^{\phi}$.

It follows that $\psi(\mathbf{b}) \bullet \mathbf{y}=\psi(\mathbf{b}) \bullet \mathbf{x}$. Thus, $\mathbf{y} \in F_{\psi}(\mathbf{b})$, because $\mathbf{x} \in F_{\psi}(\mathbf{b}) . \quad \diamond$ claim 4

Proposition C.2 and Claim 4 imply that $F_{\psi}=F_{\phi}$, as desired. 
The next result is used to prove Proposition 6.3, but is also of independent interest. For any $\mu \in \Delta(\mathcal{X})$, let $\mathcal{K}_{R}(\mu):=\left\{k \in \mathcal{K} ;\left|\widetilde{\mu}_{k}\right| \geq R\right\}$. For any $\mathbf{x} \in \mathcal{X}$, let $\mathcal{K}_{R}(\mu, \mathbf{x}):=\{k \in$ $\left.\mathcal{K}_{R}(\mu) ; \quad x_{k}=\operatorname{Maj}_{k}(\mu)\right\}$. The $R$-core of $\mu$ is the (possibly empty) set $\mathcal{X}_{R}(\mu):=\{\mathbf{x} \in \mathcal{X}$; $x_{k}=\operatorname{Maj}(\mu)_{k}$ for all $\left.k \in \mathcal{K}_{R}(\mu)\right\}$; the set of all elements of $\mathcal{X}$ which agree with every majority of size $(R+1) / 2$.

Lemma E.6 Let $\phi:[-1,1] \longrightarrow{ }^{*} \mathbb{R}$ be a gain function. Let $R \in(0,1)$, and suppose that $\phi$ is continuous, real-valued, and unbounded on $(-R, R)$, and $\phi$ is infinite on $[-1,-R] \sqcup[R, 1]$.

(a) For any $\mathbf{y} \in F_{\phi}(\mu)$, and any other $\mathbf{x} \in \mathcal{X}$, we have $\mathcal{K}_{R}(\mu, \mathbf{x}) \subseteq \mathcal{K}_{R}(\mu, \mathbf{y})$.

(b) If $F_{\phi}$ is not upper hemicontinuous at $\mu$, then $\mathcal{X}_{R}(\mu)=\emptyset$.

(c) Conversely, if $\mathcal{X}_{R}(\mu) \neq \emptyset$, then $F_{\phi}(\mu) \subseteq \mathcal{X}_{R}(\mu)$, and $F_{\phi}$ is upper hemicontinuous at $\mu$.

Proof: (a) (by contrapositive) Let $\mathbf{x}, \mathbf{y} \in \mathcal{X}$, and suppose $\mathcal{K}_{R}(\mu, \mathbf{x}) \supset \mathcal{K}_{R}(\mu, \mathbf{y})$. Then $\mathcal{K}_{ \pm}(\mathbf{x}, \mathbf{y}) \cap \mathcal{K}_{R}(\mu) \neq \emptyset$. For all $k \in \mathcal{K}_{ \pm}(\mathbf{x}, \mathbf{y}) \cap \mathcal{K}_{R}(\mu)$, we have $\left|\widetilde{\mu}_{k}\right| \geq R$, so that $\left|\phi\left(\widetilde{\mu}_{k}\right)\right|=\infty$; meanwhile, $x_{k}=\operatorname{Maj}_{k}(\mu)=-y_{k}$, so that $\left(x_{k}-y_{k}\right) \phi\left(\widetilde{\mu}_{k}\right)=\infty$. Meanwhile, for all $k \in \mathcal{K}_{ \pm}(\mathbf{x}, \mathbf{y}) \backslash \mathcal{K}_{R}(\mu)$, the value of $\left(x_{k}-y_{k}\right) \phi\left(\widetilde{\mu}_{k}\right)$ is finite. Thus, $(\mathbf{x}-\mathbf{y}) \bullet \phi(\widetilde{\mu})$ is (positive) infinite. Thus, $\mathbf{y} \notin F_{\phi}(\mu)$.

By contrapositive, if $\mathbf{y} \in F_{\phi}(\mu)$, then no such $\mathbf{x} \in \mathcal{X}$ can exist.

(b) Suppose $F_{\phi}$ is not upper hemicontinuous at $\mu$; then there exists some sequence $\left\{\mu^{n}\right\}_{n=1}^{\infty}$ such that $\mu^{n} \underset{n \rightarrow \infty}{\longrightarrow} \mu$, and some $\mathbf{x} \in \mathcal{X}$ such that $\mathbf{x} \in F_{\phi}\left(\mu^{n}\right)$ for all $n \in \mathbb{N}$, but $\mathbf{x} \notin F_{\phi}(\mu)$.

Claim 1: For any other $\mathbf{z} \in \mathcal{X}$, we have $\mathcal{K}_{R}(\mu, \mathbf{z}) \subseteq \mathcal{K}_{R}(\mu, \mathbf{x})$.

Proof: By contradiction, suppose there was some $\mathbf{z} \in \mathcal{X}$ with $\mathcal{K}_{R}(\mu, \mathbf{z}) \supset \mathcal{K}_{R}(\mu, \mathbf{x})$. Then $\mathcal{K}_{ \pm}(\mathbf{x}, \mathbf{z}) \cap \mathcal{K}_{R}(\mu) \neq \emptyset$. For all $k \in \mathcal{K}_{ \pm}(\mathbf{x}, \mathbf{z}) \cap \mathcal{K}_{R}(\mu)$, we have $\widetilde{\mu}_{k}^{n} \underset{n \rightarrow \infty}{\longrightarrow} \widetilde{\mu}_{k}$, and hence $\left|\phi\left(\widetilde{\mu}_{k}^{n}\right)\right| \underset{n \rightarrow \infty}{\longrightarrow} \infty$. Meanwhile, $z_{k}=\operatorname{Maj}_{k}(\mu)=-x_{k}$, so that $\left(z_{k}-x_{k}\right) \phi\left(\widetilde{\mu}_{k}^{n}\right) \underset{n \rightarrow \infty}{\longrightarrow} \infty$.

On the other hand, for all $k \in \mathcal{K}_{ \pm}(\mathbf{x}, \mathbf{y}) \backslash \mathcal{K}_{R}(\mu)$, there is some $R_{k}<R$ such that $\left|\widetilde{\mu}_{k}^{n}\right| \leq$ $R_{k}$ for all large enough $n \in \mathbb{N}$; thus, there is some $M_{k}<\infty$ such that $\left|\left(x_{k}-y_{k}\right) \phi\left(\widetilde{\mu}_{k}^{n}\right)\right|<$ $M_{k}$ for all large enough $n \in \mathbb{N}$. Thus, if $n$ is large enough, then $\mathbf{z} \bullet \phi\left(\widetilde{\mu}^{n}\right)>\mathbf{x} \bullet \phi\left(\widetilde{\mu}^{n}\right)$, which means $\mathbf{x} \notin F_{\phi}\left(\mu^{n}\right)$. Contradiction.

$\diamond$ Claim 1

Now, by contradiction, suppose that $\mathcal{X}_{R}(\mu) \neq \emptyset$. Then Claim 1 implies that $\mathbf{x} \in \mathcal{X}_{R}(\mu)$. Meanwhile, if $\mathbf{y} \in F_{\phi}(\mu)$, then part (a) implies that $\mathbf{y} \in \mathcal{X}_{R}(\mu)$. Thus, $\mathcal{K}_{ \pm}(\mathbf{x}, \mathbf{y}) \subseteq$ $\mathcal{K} \backslash \mathcal{K}_{R}(\mu)$. Thus,

$$
\begin{aligned}
(\mathbf{x}-\mathbf{y}) \bullet \phi(\widetilde{\mu}) & =\sum_{k \in \mathcal{K}_{ \pm}(\mathbf{x}, \mathbf{y})}\left(x_{k}-y_{k}\right) \phi\left(\widetilde{\mu}_{k}\right)=\sum_{\mathcal{K} \backslash \mathcal{K}_{R}(\mu)}\left(x_{k}-y_{k}\right) \phi\left(\widetilde{\mu}_{k}\right) \\
& \overline{\overline{(\diamond)}} \sum_{\mathcal{K} \backslash \mathcal{K}_{R}(\mu)}\left(x_{k}-y_{k}\right) \lim _{n \rightarrow \infty} \phi\left(\widetilde{\mu}_{k}^{n}\right)=\lim _{n \rightarrow \infty} \sum_{\mathcal{K} \backslash \mathcal{K}_{R}(\mu)}\left(x_{k}-y_{k}\right) \phi\left(\widetilde{\mu}_{k}^{n}\right) \\
& =\lim _{n \rightarrow \infty} \sum_{k \in \mathcal{K}_{ \pm}(\mathbf{x}, \mathbf{y})}\left(x_{k}-y_{k}\right) \phi\left(\widetilde{\mu}_{k}^{n}\right)=\lim _{n \rightarrow \infty}(\mathbf{x}-\mathbf{y}) \bullet \phi\left(\widetilde{\mu}^{n}\right) \underset{(*)}{\geq} 0 .
\end{aligned}
$$


Here, $(\diamond)$ is because $\widetilde{\mu}_{k}^{n} \longrightarrow{ }_{n \rightarrow \infty} \widetilde{\mu}_{k} \in(R, R)$ for all $k \in \mathcal{K} \backslash \mathcal{K}_{R}(\mu)$, and $\phi$ is continuous on $(-R, R)$ by hypothesis. Meanwhile, $(*)$ is because $(\mathbf{x}-\mathbf{y}) \bullet \phi\left(\widetilde{\mu}^{n}\right) \geq 0$ for all $n \in \mathbb{N}$, because $\mathbf{x} \in F_{\phi}\left(\mu^{n}\right)$ for all $n \in \mathbb{N}$.

We conclude that $\mathbf{x} \bullet \phi(\widetilde{\mu}) \geq \mathbf{y} \bullet \phi(\widetilde{\mu})$; thus, $\mathbf{x} \in F_{\phi}(\mu)$, because $\mathbf{y} \in F_{\phi}(\mu)$. Contradiction.

(c) follows from (a) and the contrapositive of (b).

Proof of Proposition 6.3. (by contradiction) Let $\mu \in \Delta(\mathcal{X})$, and suppose $F_{\phi}$ is not upper hemicontinuous at $\mu$. Let $R:=1-\frac{2}{M}$, and let $\mathcal{K}_{R}(\mu):=\left\{k \in \mathcal{K} ;\left|\widetilde{\mu}_{k}\right| \geq R\right\}$. For any subset $\mathcal{J} \subseteq \mathcal{K}$, let $\mathcal{X}_{\mathcal{J}}(\mu):=\left\{\mathbf{x} \in \mathcal{X}_{\mathcal{A}}^{\mathrm{pr}} ; \mathbf{x}_{\mathcal{J}}=\operatorname{Maj}(\mu)_{\mathcal{J}}\right\}$.

[Definition of forbidden/critical words. Fact: Any critical word in $\mathcal{X}_{\mathcal{A}}^{\mathrm{pr}}$ represents a 'preference cycle'; maximum length of such a critical word is $M]$.

Claim 1: $\quad$ (a) $\left|\mathcal{K}_{R}(\mu)\right| \geq M$

(b) Any critical subword of $\operatorname{Maj}(\mu)_{\mathcal{K}_{R}(\mu)}$ is a Condorcet cycle of length $M$.

(c) $\mu\left[\mathcal{X}_{k}(\mu)\right]=1-\frac{1}{M}$ for all $k \in \mathcal{K}_{R}(\mu)$.

Proof: For any $k \in \mathcal{K}_{R}(\mu)$, we must have $\mu\left[\mathcal{X}_{k}(\mu)\right] \geq 1-\frac{1}{M}$. Thus, for any subset $\mathcal{J} \subseteq \mathcal{K}_{R}(\mu)$, we have

$$
\mu\left[\mathcal{X}_{\mathcal{J}}(\mu)\right] \geq 1-\frac{|\mathcal{J}|}{M}
$$

(a) If $F_{\phi}$ is not upper hemicontinuous at $\mu$, and $\mathcal{J}:=\mathcal{K}_{R}(\mu)$, then Lemma E.6(b) says $\mathcal{X}_{\mathcal{J}}(\mu)=\emptyset$, which means that $\mu\left[\mathcal{X}_{\mathcal{J}}(\mu)\right]=0$. Thus, inequality (E9) says that $\left|\mathcal{K}_{R}(\mu)\right| \geq M$.

(b) If $|\mathcal{J}|<M$, then inequality (E9) implies that $\mu\left[\mathcal{X}_{\mathcal{J}}(\mu)\right]>0$; hence $\mathcal{X}_{\mathcal{J}}(\mu) \neq \emptyset$. Thus, any subword of less than $M$ coordinates of $\operatorname{Maj}(\mu)$ is $\mathcal{X}_{\mathcal{A}}^{\mathrm{pr}}$-admissible. Thus, the smallest forbidden words (i.e. the critical words) in $\operatorname{Maj}(\mu)_{\mathcal{K}_{R}(\mu)}$ must have length $M$. Such a critical word in $\mathcal{X}_{\mathcal{A}}^{\mathrm{pr}}$ has the form of a Condorcet cycle of length $M$.

(c) (By contradiction) Suppose instead that there was some $k \in \mathcal{K}$ with $\mu\left[\mathcal{X}_{k}(\mu)\right]>1-\frac{1}{M}$. By reasoning similar to inequality (E9), we conclude that, for any $\mathcal{J} \subset \mathcal{K}_{R}(\mu)$ with $|\mathcal{J}|=M$, we have $\mu\left[\mathcal{X}_{\mathcal{J}}(\mu)\right]>1-\frac{M}{M}=0$. Thus, $\mathcal{X}_{\mathcal{J}}(\mu) \neq \emptyset$. Thus, $\operatorname{Maj}(\mu)_{\mathcal{J}}$ is not a $\mathcal{X}_{\mathcal{A}}^{\mathrm{pr}}$-forbidden word. Thus, the smallest forbidden word containing $k$ must have length greater than $M$, which means there is a critical word in $\mathcal{X}_{\mathcal{A}}^{\mathrm{pr}}$ of length greater than $M$, which is false.

$\diamond$ Claim 1

Let $\mathcal{J} \subseteq \mathcal{K}$ be a subset with $|\mathcal{J}|=M$, such that $\operatorname{Maj}(\mu)_{\mathcal{J}}$ is a maximal Condorcet cycle; say it represents the cycle $\left(a_{1} \succ a_{2} \succ \cdots \succ a_{M} \succ a_{1}\right)$. For any $i \in \mathcal{J}$, if coordinate $i$ represents the link " $a_{n} \succ a_{n+1}$ ", then let $\mathbf{x}_{i} \in \mathcal{X}_{\mathcal{A}}^{\mathrm{pr}}$ represent the preference order obtained by breaking the Condorcet cycle at this link (i.e. $a_{n+1} \succ a_{n+2} \succ \cdots a_{M} \succ a_{1} \succ$ $\left.a_{2} \succ \cdots \succ a_{n}\right)$. Let $\mathcal{X}^{\prime}:=\left\{\mathbf{x}_{1}, \mathbf{x}_{2}, \ldots, \mathbf{x}_{M}\right\}$. It follows that $\mathcal{X}_{i}(\mu)=\mathcal{X}^{\prime} \backslash\left\{\mathbf{x}_{i}\right\}$ (a set of $M-1$ elements).

Claim $1(\mathrm{c})$ says that $\mu\left[\mathcal{X}_{i}(\mu)\right]=1-\frac{1}{M}$ for all $i \in \mathcal{J}$. From this, it follows that $\mu\left[\mathbf{x}_{i}\right]=\frac{1}{M}$ for all $i \in \mathcal{J}$. From this, it is easy to deduce that $\operatorname{SME}\left(\mathcal{X}_{\mathcal{A}}^{\mathrm{pr}}, \mu\right)=\mathcal{X}^{\prime}$. Furthermore, from 
the symmetry of the profile $\mu$, we deduce that $\phi(\widetilde{\mu}) \bullet \mathbf{x}_{i}=\phi(\widetilde{\mu}) \bullet \mathbf{x}_{j}$ for all $i, j \in[1 \ldots M]$; thus, $F_{\phi}(\mu)=\mathcal{X}^{\prime}$.

However, if $F_{\phi}$ is not upper hemicontinuous at $\mu$; then there exists some sequence $\left\{\mu^{n}\right\}_{n=1}^{\infty}$ such that $\mu^{n} \underset{n \rightarrow \infty}{\longrightarrow} \mu$, and some $\mathbf{x} \in \mathcal{X}$ such that $\mathbf{x} \in F_{\phi}\left(\mu^{n}\right)$ for all $n \in \mathbb{N}$, but $\mathbf{x} \notin F_{\phi}(\mu)$. Then Claim 1 in the proof of Lemma E.6(a) implies that $\mathbf{x} \in \mathcal{X}^{\prime}$. Thus, $\mathbf{x} \in F_{\phi}(\mu)$. Contradiction.

Theorem 6.4 is actually a special case of a more general result. Let $\phi, \psi:[-1,1] \longrightarrow \mathbb{R}$ be odd gain functions, and let $\mathcal{X}$ be a judgement space. We say $\psi$ is an $\mathcal{X}$-compatible affine transform of $\phi$ if there exist functions $s: \mathcal{R}_{\mathcal{X}}^{\phi} \longrightarrow \mathbb{R}_{+}$and $t: \mathcal{R}_{\mathcal{X}}^{\phi} \longrightarrow \mathbb{R}$ such that:

(C1) $s$ is constant on each $F_{\phi}$-pivotal class of $\mathcal{R}_{\mathcal{X}}^{\phi}$.

(C2) $t$ is constant on each connected component of $\mathcal{R}_{\mathcal{X}}^{\phi}$.

(C3) For all $\mathbf{x}, \mathbf{y} \in \mathcal{X}$ with $d(\mathbf{x}, \mathbf{y}) \geq 3$, and all $\mathbf{b} \in{ }^{o} \mathcal{B}_{\mathbf{x}, \mathbf{y}}^{\phi}, \sum_{k \in \mathcal{K}_{ \pm}(\mathbf{x}, \mathbf{y})}\left(x_{k}-y_{k}\right) t\left(b_{k}\right)=0$.

(C4) For all $r \in \mathcal{R}_{\mathcal{X}}^{\phi}$, we have $\psi(r)=s(r) \cdot \phi(r)+t(r)$.

If $\phi$ and $\psi$ are odd and continuous, then (C4) holds at all points in the closure of $\mathcal{R}_{\mathcal{X}}^{\phi}$; thus (C2) forces $t$ to be constantly zero on any connected component of $\mathcal{R}_{\mathcal{X}}^{\phi}$ which has 0 in its closure. If all of $\mathcal{R}_{\mathcal{X}}^{\phi}$ is in one $F_{\phi}$-pivotal class, then (C1) means that $s$ is a constant. If $\mathcal{R}_{\mathcal{X}}^{\phi}$ is connected, then (C1) and (C2) make both $s$ and $t$ constant functions, and then (C3) forces $t \equiv 0$, so $\psi$ is just a scalar multiple of $\phi$ on $\mathcal{R}_{\mathcal{X}}^{\phi}$. Even if $\mathcal{R}_{\mathcal{X}}^{\phi}$ is not connected, (C3) requires the (piecewise constant) values of $t$ to satisfy a large, homogeneous system of linear equations, which will usually force $t \equiv 0$; in this case, $\psi$ is a 'piecewise constant scalar multiple' of $\phi$, with possibly different scalars on different $F_{\phi}$-pivotal classes of $\mathcal{R}_{\mathcal{X}}^{\phi}$.

Theorem E.7 Let $\phi, \psi:[-1,1] \longrightarrow \mathbb{R}$ be odd, real-valued gain functions, and let $\mathcal{X}$ be a judgement space. Suppose $F_{\phi}$ and $F_{\psi}$ are upper hemicontinuous on $\Delta(\mathcal{X})$.

(a) If $\psi$ is any $\mathcal{X}$-compatible affine transform of $\phi$, then $F_{\phi}(\mathcal{X}, \mu)=F_{\psi}(\mathcal{X}, \mu)$ for all $\mu \in \Delta(\mathcal{X})$.

(b) Conversely, suppose $\mathcal{X}$ is thick and $\phi$ and $\psi$ are continuous on $\mathcal{R}_{\mathcal{X}}^{F}$. If $F_{\phi}(\mathcal{X}, \mu)=$ $F_{\psi}(\mathcal{X}, \mu)$ for all $\mu \in \Delta(\mathcal{X})$, then $\psi$ is an $\mathcal{X}$-compatible affine transform of $\phi$.

Proof of Theorem E.7(a). Let $\mathcal{C}:=\operatorname{conv}(\mathcal{X})$.

Claim 1: $\quad$ For any $\mathbf{x}, \mathbf{y} \in \mathcal{X}$, if $d(\mathbf{x}, \mathbf{y}) \geq 3$, then ${ }^{o} \mathcal{B}_{\mathbf{x}, \mathbf{y}}^{\phi} \subseteq \mathcal{B}_{\mathbf{x}, \mathbf{y}}^{\psi} \cup\left(\mathcal{C} \backslash \mathcal{C}_{\mathbf{x}}^{\psi}\right)$. 
Proof: Let $s: \mathcal{R}_{\mathcal{X}}^{\phi} \longrightarrow \mathbb{R}_{+}$and $t: \mathcal{R}_{\mathcal{X}}^{\phi} \longrightarrow \mathbb{R}$ satisfy $(\mathrm{C} 1)-(\mathrm{C} 4)$, and let $\mathbf{b} \in{ }^{\circ} \mathcal{B}_{\mathbf{x}, \mathbf{y}}^{\phi}$. Then

$$
\begin{aligned}
& (\mathbf{x}-\mathbf{y}) \bullet \psi(\mathbf{b})=\sum_{k \in \mathcal{K}_{ \pm}(\mathbf{x}, \mathbf{y})}\left(x_{k}-y_{k}\right) \psi\left(b_{k}\right) \\
& \overline{\overline{(*)}} \sum_{k \in \mathcal{K}_{ \pm}(\mathbf{x}, \mathbf{y})}\left(x_{k}-y_{k}\right)\left(s\left(b_{k}\right) \phi\left(b_{k}\right)+t\left(b_{k}\right)\right) \\
& \overline{\overline{(\dagger)}} s \sum_{k \in \mathcal{K}_{ \pm}(\mathbf{x}, \mathbf{y})}\left(x_{k}-y_{k}\right)\left(\phi\left(b_{k}\right)+t\left(b_{k}\right)\right) \\
& =s(\mathbf{x}-\mathbf{y}) \bullet \phi(\mathbf{b})+\sum_{k \in \mathcal{K}_{ \pm}(\mathbf{x}, \mathbf{y})}\left(x_{k}-y_{k}\right) t\left(b_{k}\right) \\
& \overline{\overline{(\dagger)}} 0+\sum_{k \in \mathcal{K}_{ \pm}(\mathbf{x}, \mathbf{y})}\left(x_{k}-y_{k}\right) t\left(b_{k}\right) \overline{\overline{(\ddagger)}} 0 .
\end{aligned}
$$

Here, $(*)$ is by $(\mathrm{C} 4)$. Next, $(\diamond)$ is because for all $j, k \in \mathcal{K}_{ \pm}(\mathbf{x}, \mathbf{y})$, we have $b_{j}, b_{k} \in \mathcal{R}_{\mathcal{X}}^{\phi}$, and $b_{j} \sim b_{k}$, so that (C1) yields some $s>0$ such that $s\left(b_{k}\right)=s$ for all $k \in \mathcal{K}_{ \pm}(\mathbf{x}, \mathbf{y})$. Finally, $(\dagger)$ is because $\mathbf{b} \in{ }^{\circ} \mathcal{B}_{\mathbf{x}, \mathbf{y}}^{\phi}$, and $(\ddagger)$ is by (C3).

If $(\mathbf{x}-\mathbf{z}) \bullet \psi(\mathbf{b}) \geq 0$ for all $\mathbf{z} \in \mathcal{X}$, then statement (E10) implies that $F_{\psi}(\mathbf{b}) \supseteq\{\mathbf{x}, \mathbf{y}\}$, so $\mathbf{b} \in \mathcal{B}_{\mathbf{x}, \mathbf{y}}^{\psi}$. Otherwise, if $(\mathbf{x}-\mathbf{z}) \bullet \psi(\mathbf{b})<0$ for some $\mathbf{z} \in \mathcal{X}$, then $\mathbf{x} \notin F_{\psi}(\mathbf{b})$, so $\mathrm{b} \in \mathcal{C} \backslash \mathcal{C}_{\mathbf{x}}^{\psi}$.

$\diamond$ Claim 1

Now Proposition C.2 and Claim 1 imply that $F_{\phi}(\mathcal{X}, \mu)=F_{\psi}(\mathcal{X}, \mu)$ for all $\mu \in \Delta(\mathcal{X})$.

To prove Theorem E.7(b), we must first generalize a well-known result about solutions to the Cauchy functional equation. Let $\mathcal{W} \subseteq[-1,1]$ be an open subset, and let $\phi: \mathcal{W} \longrightarrow \mathbb{R}$. We say $\phi$ has constant slope on $\mathcal{W}$ if there is some $s \in \mathbb{R}$, and, for each connected component $\mathcal{W}^{\prime} \subseteq \mathcal{W}$, there is some constant $t \in \mathbb{R}$ such that $\phi(w)=s w+t$ for all $w \in \mathcal{W}^{\prime}$. (In particular, $\phi^{\prime}(w)=s$, for all $w \in \mathcal{W}$.)

Lemma E.8 Let $N \geq 2$, and let $\mathcal{U} \subseteq \mathbb{R}^{N}$ be a connected open subset. Let $\mathcal{V}:=\left\{u_{1}+u_{2}+\right.$ $\left.\cdots+u_{N} ; \mathbf{u} \in \mathcal{U}\right\}$. For all $n \in \mathcal{N}$, let $\mathcal{U}_{n}:=\pi_{n}(\mathcal{U})$, and let $\mathcal{W}:=\mathcal{V} \cup \mathcal{U}_{1} \cup \cdots \cup \mathcal{U}_{N} \subseteq \mathbb{R}$. Let $\tau: \mathcal{W} \longrightarrow \mathbb{R}$ be a continuous, increasing function, such that $\tau\left(u_{1}+u_{2}+\cdots+u_{N}\right)=$ $\tau\left(u_{1}\right)+\cdots+\tau\left(u_{N}\right)$ for all $\mathbf{u} \in \mathcal{U}$. Then $\phi$ has constant slope $s>0$ on $\mathcal{W}$.

Proof: For any $q \in \mathcal{U}_{1}$, let $\mathcal{V}_{q}:=\left\{u_{1}+u_{2}+\cdots+u_{N} ; \mathbf{u} \in \mathcal{U}\right.$ and $\left.u_{1}=q\right\}$. Note that $\mathcal{U}_{1}$ is an open subset of $\mathbb{R}$ (because $\mathcal{U}$ is an open subset of $\mathbb{R}^{N}$ ).

Claim 1: (a) For any $q \in \mathcal{U}_{1}$ and $v \in \mathcal{V}_{q}$, there exists some $\epsilon>0$ such that $v \in \mathcal{V}_{q^{\prime}}$ for all $q^{\prime} \in(q-\epsilon, q+\epsilon)$.

(b) If $\mathcal{Q} \subseteq \mathcal{U}_{1}$ is any dense subset, then $\bigcup_{q \in \mathcal{Q}} \mathcal{V}_{q}=\mathcal{V}$.

Proof: (a) Find $\mathbf{u} \in \mathcal{U}$ with $u_{1}=q$ and $u_{2}+\cdots+u_{N}=v$. For any $\epsilon>0$, let $\mathcal{B}(\mathbf{u}, \epsilon):=\left\{\mathbf{r} \in \mathbb{R}^{N} ;\left|r_{n}-u_{n}\right|<\epsilon\right.$, for all $\left.n \in[1 \ldots N]\right\}$ (the $\epsilon$-ball in the $\infty$-norm ). There exists some $\epsilon>0$ such that $\mathcal{B}(\mathbf{u}, \epsilon) \subset \mathcal{U}$ (because $\mathcal{U}$ is open). Let $q^{\prime} \in(q-\epsilon, q+\epsilon)$. 
Define $\mathbf{u}^{\prime}$ by $u_{1}^{\prime}=q^{\prime}$ and $u_{2}^{\prime}=u_{2}+\left(q-q^{\prime}\right)$, while $u_{n}^{\prime}=u_{n}$ for $n \in[3 \ldots N]$. Then $\mathbf{u}^{\prime} \in \mathcal{B}(\mathbf{u}, \epsilon)$, so $\mathbf{u}^{\prime} \in \mathcal{U}$. But by construction, $u_{1}^{\prime}=q^{\prime}$ and $u_{1}^{\prime}+\cdots+u_{N}^{\prime}=v$. Thus, $v \in \mathcal{V}_{q^{\prime}}$.

(b) From their definitions it is clear that $\bigcup_{q \in \mathcal{U}} \mathcal{V}_{q}=\mathcal{V}$. Part (a) implies that it suffices to take the union over a dense subset of $\mathcal{U}$.

$\diamond$ Claim 1

Claim 2: $\quad$ Let $q \in \mathcal{U}_{1}$. Suppose $\tau$ is differentiable at $q$, and $\tau^{\prime}(q)=s$. Then $\tau^{\prime}(v)=s$ for all $v \in \mathcal{V}_{q}$.

Proof: Let $v \in \mathcal{V}_{q}$; then $v=u_{1}+u_{2}+\cdots+u_{N}$ for some $\mathbf{u} \in \mathcal{U}$ with $u_{1}=q$. If $\epsilon$ is small enough, then $\left(u_{1}+\epsilon, u_{2}, \ldots, u_{N}\right)$ is also in $\mathcal{U}$ (because $\mathcal{U}$ is open). Thus

$$
\begin{aligned}
\tau(v+\epsilon)-\tau(v) & =\tau\left(u_{1}+\epsilon+u_{2}+\cdots+u_{N}\right)-\tau\left(u_{1}+u_{2}+\cdots+u_{N}\right) \\
& \overline{(*)}\left(\tau\left(u_{1}+\epsilon\right)+\tau\left(u_{2}\right)+\cdots+\tau\left(u_{N}\right)\right)-\left(\tau\left(u_{1}\right)+\tau\left(u_{2}\right)+\cdots+\tau\left(u_{N}\right)\right) \\
& =\tau\left(u_{1}+\epsilon\right)-\tau\left(u_{1}\right)=\tau(q+\epsilon)-\tau(q) . \\
\text { Thus, } \tau^{\prime}(v) & =\lim _{\epsilon \rightarrow 0} \frac{\tau(v+\epsilon)-\tau(v)}{\epsilon}=\lim _{\epsilon \rightarrow 0} \frac{\tau(q+\epsilon)-\tau(q)}{\epsilon}=\tau^{\prime}(q)=s,
\end{aligned}
$$
as desired. Here, $(*)$ is by the hypothesis of the theorem.

Claim 3: $\quad$ There exists some constant $s \geq 0$ such that $\tau^{\prime}(v)=s$ for all $v \in \mathcal{V}$.

Proof: Since $\tau$ is nondecreasing and $\mathcal{U}_{1}$ is open, there is a dense subset $\mathcal{Q} \subset \mathcal{U}_{1}$ such that $\tau^{\prime}(q)$ exists for all $q \in \mathcal{Q}$ (Kolmogorov and Fomīn, 1975, Thm.6, §31.2, p.321). Thus, Claim 2 says that $\tau^{\prime}(v)$ exists for all $v \in \bigcup_{q \in \mathcal{Q}} \mathcal{V}_{q}$, and is constant on $\mathcal{V}_{q}$ for each $q \in \mathcal{Q}$. Claim 1(a) says that $\mathcal{V}_{q_{1}}$ overlaps $\mathcal{V}_{q_{2}}$ if $q_{1}$ and $q_{2}$ are close; thus, $\tau^{\prime}$ is constant on $\mathcal{V}_{q_{1}} \cup \mathcal{V}_{q_{2}}$. Thus, $\tau^{\prime}$ must be constant on each connected component of the union $\bigcup_{q \in \mathcal{Q}} \mathcal{V}_{q}$. But Claim $1(\mathrm{~b})$ says this union is all of $\mathcal{V}$, which is connected (because $\mathcal{U}$ is connected). Thus, $\tau^{\prime}$ exists and is constant on $\mathcal{V}$.

Claim 3

Claim 4: $\quad \tau^{\prime}(u)=s$ for all $u \in \mathcal{U}_{1}$.

Proof: Let $v \in \mathcal{V}_{u}$; then $v=u_{1}+u_{2}+\cdots+u_{N}$ for some $\mathbf{u} \in \mathcal{U}$ with $u_{1}=u$. If $\epsilon$ is small enough, then $\left(u_{1}+\epsilon, u_{2}, \ldots, u_{N}\right)$ is also in $\mathcal{U}$ (because $\mathcal{U}$ is open). Then, by exactly the same reasoning as Claim 2, we have $\tau^{\prime}(u)=\tau^{\prime}(v)$. But $\tau^{\prime}(v)=s$, by Claim 3 . $\diamond$ Claim 4

For any $n \in[1 \ldots N]$, a similar argument to Claim 4 shows that $\tau^{\prime}(u)=s$ for all $u \in \mathcal{U}_{n}$. Thus, $\tau^{\prime}(w)=s$ for all $w \in \mathcal{W}$. Also, $s \geq 0$ since $\tau$ is nondecreasing. Thus, if $\mathcal{W}^{\prime} \subseteq \mathcal{W}$ is any connected component, then $\tau$ is an affine function on $\mathcal{W}^{\prime}$. That is, there exists $t \in \mathbb{R}$ such that $\tau(w)=s w+t$ for all $w \in \mathcal{W}^{\prime}$. 
Proof of Theorem E.7(b). Let $\mathbf{x}, \mathbf{y} \in \mathcal{X}$, with $d(\mathbf{x}, \mathbf{y}) \geq 3$. We will first show that $\phi$ is an affine transformation of $\psi$ in an open neighbourhood of each point in the set $\mathcal{R}_{\mathbf{x}, \mathbf{y}}:=\bigcup_{k \in \mathcal{K}_{ \pm}(\mathbf{x}, \mathbf{y})} \mathcal{R}_{\mathbf{x}, \mathbf{y}}^{k}$.

Suppose that ${ }^{\circ} \mathcal{B}_{\mathbf{x}, \mathbf{y}}^{\phi} \neq \emptyset$ (otherwise $\mathcal{R}_{\mathbf{x}, \mathbf{y}}=\emptyset$ and there is nothing to prove). Let $\mathcal{R}^{\prime}:=$ $\phi\left(\mathcal{R}_{\mathbf{x}, \mathbf{y}}\right)$ and $\tau:=\psi \circ \phi^{-1}$; it is equivalent to show that $\tau$ is locally affine on $\mathcal{R}^{\prime}$.

Without loss of generality, suppose $\mathcal{K}_{ \pm}(\mathbf{x}, \mathbf{y})=[1 \ldots J]$ for some $J \geq 3$. Also suppose $x_{1}=$ 1 and $y_{1}=-1$, whereas $x_{j}=-1$ and $y_{j}=1$ for all $j \in[2 \ldots J]$. Let $\mathcal{O}:=\left(\mathcal{R}_{\mathcal{X}}^{\phi}\right)^{J} \times \mathbb{R}^{K-J}$; then $\mathcal{O}$ is an open subset of $\mathbb{R}^{\mathcal{K}}$ (because Lemma E.4(d) says $\mathcal{R}_{\mathcal{X}}^{\phi}$ is open, because $F_{\phi}$ is upper hemicontinuous and $\mathcal{X}$ is thick). Observe that ${ }^{\circ} \mathcal{B}_{\mathbf{x}, \mathbf{y}}^{F} \subseteq \mathcal{O}$ (because $\pi_{j}\left({ }^{\circ} \mathcal{B}_{\mathbf{x}, \mathbf{y}}^{F}\right) \subseteq \mathcal{R}_{\mathcal{X}}^{\phi}$ for all $j \in[1 \ldots J]$, by definition of $\mathcal{R}_{\mathcal{X}}^{\phi}$ ). Let

$$
\mathcal{C}^{\prime}:=(\mathcal{O} \cap \operatorname{int}(\mathcal{C})) \backslash \underset{\mathbf{z} \in \mathcal{X} \backslash\{\mathbf{x}, \mathbf{y}\}}{\bigcup} \mathcal{C}_{\mathbf{z}} .
$$

Then $\mathcal{C}^{\prime}$ is an open subset of $\mathbb{R}^{\mathcal{K}}$ (because $\mathcal{O}$ and $\operatorname{int}(\mathcal{C})$ are open, while $\mathcal{C}_{\mathbf{z}}$ is closed for all $\mathbf{z} \in \mathcal{X}$, because $F_{\phi}$ is upper hemicontinuous). We have ${ }^{\circ} \mathcal{B}_{\mathbf{x}, \mathbf{y}}^{F}=\left\{\mathbf{c} \in \mathcal{C}^{\prime}\right.$; $(\mathbf{x}-\mathbf{y}) \bullet \phi(\mathbf{c})=0\}$. By our assumption on the coordinates of $\mathbf{x}$ and $\mathbf{y}$, this means

$$
{ }^{{ }^{\circ} \mathcal{B}_{\mathbf{x}, \mathbf{y}}^{F}}=\left\{\mathbf{c} \in \mathcal{C}^{\prime} ; \phi\left(c_{1}\right)=\sum_{j=2}^{J} \phi\left(c_{j}\right)\right\} .
$$

Claim 1: Let $\widetilde{\mathcal{R}}_{\mathcal{X}}^{\phi}:=\phi\left(\mathcal{R}_{\mathcal{X}}^{\phi}\right) \subseteq \mathbb{R}$. Then $\phi: \mathcal{R}_{\mathcal{X}}^{F} \longrightarrow \widetilde{\mathcal{R}}_{\mathcal{X}}^{\phi}$ is a homeomorphism.

Proof: $\phi$ is injective because $\phi$ is increasing on $\mathcal{R}_{\mathcal{X}}^{\phi}$ by hypothesis. Also, $\phi$ is continuous on $\mathcal{R}_{\mathcal{X}}^{\phi}$ by hypothesis. It remains to show that $\phi$ is an open map on $\mathcal{R}_{\mathcal{X}}^{\phi}$. To see this, let $\mathcal{S} \subset \mathcal{R}_{\mathcal{X}}^{\phi}$ be a relatively open subset; we must show that $\phi(\mathcal{S})$ is open. Let $t \in \phi(\mathcal{S})$; then $t=\phi(s)$ for some $s \in \mathcal{S}$. Since $\mathcal{S}$ is relatively open in $\mathcal{R}_{\mathcal{X}}^{\phi}$, and $\mathcal{R}_{\mathcal{X}}^{\phi}$ is open in $\mathbb{R}$ by Lemma E.4(d), there is some $\epsilon>0$ such that $(s-\epsilon, s+\epsilon) \subseteq \mathcal{S}$. Now, $\phi(s-\epsilon)<\phi(s)<$ $\phi(s+\epsilon)$ because $\phi$ is strictly increasing. Thus, $(\phi(s-\epsilon), \phi(s+\epsilon))$ is a neighbourhood of $t=\phi(s)$. The Intermediate Value Theorem implies that $(\phi(s-\epsilon), \phi(s+\epsilon)) \subseteq \phi(\mathcal{S})$, because $\phi$ is continuous on $\mathcal{R}_{\mathcal{X}}^{F}$. Thus, $\phi(\mathcal{S})$ contains a neighbourhood around $t$. $\diamond$ Claim 1

Note that Claim 1 implies that $\tau:=\psi \circ \phi^{-1}$ is continuous on $\widetilde{\mathcal{R}}_{\mathcal{X}}^{\phi}$.

Now, define $\phi^{J}: \mathcal{O} \longrightarrow \mathbb{R}^{J}$ by $\phi^{J}(\mathbf{r}):=\left(\phi\left(r_{j}\right)\right)_{j=1}^{J}$. Claim 1 implies that $\phi^{J}$ is a continuous and open function on $\mathcal{O}$. Thus, if $\widetilde{\mathcal{C}}:=\phi^{J}\left[\mathcal{C}^{\prime}\right]$, then $\widetilde{\mathcal{C}}$ is an open subset of $\mathbb{R}^{J}$. Let $\widetilde{\mathcal{B}}_{\mathbf{x}, \mathbf{y}}:=\phi^{J}\left({ }^{\circ} \mathcal{B}_{\mathbf{x}, \mathbf{y}}^{\phi}\right)$. Then equation (E11) becomes

$$
\widetilde{\mathcal{B}}_{\mathbf{x}, \mathbf{y}}=\left\{\widetilde{\mathbf{c}} \in \widetilde{\mathcal{C}} ; \widetilde{c}_{1}=\sum_{j=2}^{J} \widetilde{c}_{j}\right\} .
$$


Let $\pi_{J}: \mathbb{R}^{J} \longrightarrow \mathbb{R}^{J-1}$ be the projection onto coordinates $[2 \ldots J]$. Then $\mathcal{B}:=\pi_{J}\left(\widetilde{\mathcal{B}}_{\mathbf{x}, \mathbf{y}}\right)$ is an open subset of $\mathbb{R}^{J-1}$, by the Open Mapping Theorem. Let $\mathcal{U} \subseteq \mathcal{B}$ be a connected component of $\mathcal{B}$, and let $\tilde{\mathcal{U}}:=\pi_{J}^{-1}(\mathcal{U}) \cap \widetilde{\mathcal{B}}_{\mathbf{x}, \mathbf{y}}$. Equation (E12) implies that

$$
\widetilde{u}_{1}=\sum_{j=2}^{J} \widetilde{u}_{j}, \quad \text { for all } \widetilde{\mathbf{u}} \in \tilde{\mathcal{U}} .
$$

So, let $\widetilde{\mathbf{u}} \in \widetilde{\mathcal{U}}$, and suppose $\widetilde{\mathbf{u}}:=\phi^{J}(\mathbf{b})$, for some $\mathbf{b} \in{ }^{\circ} \mathcal{B}_{\mathbf{x}, \mathbf{y}}$. Then $\tau(\widetilde{\mathbf{u}})=\psi(\mathbf{b})$. Furthermore, $\mathbf{b} \in{ }^{o} \mathcal{B}_{\mathbf{x}, \mathbf{y}}^{\psi}$ because $F_{\phi}(\mathbf{b})=F_{\psi}(\mathbf{b})$ by hypothesis. Thus, we have

$$
\begin{aligned}
\tau\left(\widetilde{u}_{1}\right)-\sum_{j=2}^{J} \tau\left(\widetilde{u}_{j}\right) & =\psi\left(b_{1}\right)-\sum_{j=2}^{J} \psi\left(b_{j}\right)=(\mathbf{x}-\mathbf{y}) \bullet \psi(\mathbf{b})=0 . \\
\text { Thus, } \tau\left(\widetilde{u}_{1}\right) & =\sum_{j=2}^{J} \tau\left(\widetilde{u}_{j}\right), \quad \text { for all } \widetilde{\mathbf{u}} \in \widetilde{\mathcal{U}}
\end{aligned}
$$

Combining equations (E13) and (E14), we get:

$$
\tau\left(\sum_{j=2}^{J} u_{j}\right)=\sum_{j=2}^{J} \tau\left(u_{j}\right), \quad \text { for all } \mathbf{u} \in \mathcal{U}
$$

For all $j \in[2 \ldots J]$, let $\mathcal{U}_{j}:=\pi_{j}(\mathcal{U})$. Let $\mathcal{V}:=\pi_{1}(\widetilde{\mathcal{U}})$, and let $\mathcal{W}:=\mathcal{V} \cup \mathcal{U}_{2} \cup \cdots \cup \mathcal{U}_{J}$. Then $\mathcal{W} \subseteq \widetilde{\mathcal{R}}_{\mathcal{X}}^{F}$, so Claim 1 implies that $\tau$ is continuous on $\mathcal{W}$. Also, $\tau$ is increasing, because $\psi$ and $\phi$ (and hence, $\phi^{-1}$ ) are increasing. Finally, equation (E13) says that $\mathcal{V}=\left\{u_{2}+\cdots+u_{J} ; \mathbf{u} \in \mathcal{U}\right\}$. Then equation (E15) and Lemma E.8 imply that there is some $s>0$ and, for each connected subset $\mathcal{W}^{\prime} \subseteq \mathcal{W}$, some $t \in \mathbb{R}$ such that $\tau(w)=s w+t$ for all $w \in \mathcal{W}^{\prime}$. Thus, if $\mathcal{R}^{\prime}:=\left\{\phi^{-1}(w) ; w^{\prime} \in \mathcal{W}^{\prime}\right\}$, then we have $\psi(r)=s \phi(r)+t$ for all $r \in \mathcal{R}^{\prime}$.

Proceeding in this way, we can define functions $s: \mathcal{R}_{\mathcal{X}}^{F} \longrightarrow \mathbb{R}_{+}$and $t: \mathcal{R}_{\mathcal{X}}^{F} \longrightarrow \mathbb{R}$ satisfying condition (C4). By construction, $s$ and $t$ are constant in an open neighbourhood around each point in their domain. Thus, they must be constant on each connected component of $\mathcal{R}_{\mathcal{X}}^{\phi}$. This verifies $(\mathrm{C} 2)$.

To prove $(\mathrm{C} 1)$, let $r_{1} \sim r_{2} \in \mathcal{R}_{\mathcal{X}}^{\phi}$. There exist $\mathbf{x}, \mathbf{y} \in \mathcal{X}$ with $d(\mathbf{x}, \mathbf{y}) \geq 3$, some $\mathbf{b} \in{ }^{o} \mathcal{B}_{\mathbf{x}, \mathbf{y}}^{F}$, and some $j, k \in \mathcal{K}_{ \pm}(\mathbf{x}, \mathbf{y})$ such that $b_{j}=r_{1}$ and $b_{k}=r_{2}$. But then in the above construction, if we let $\widetilde{\mathcal{U}}$ be the connected component of $\widetilde{\mathcal{B}}_{\mathbf{x}, \mathbf{y}}$ containing $\phi(\mathbf{b})$, then we end up with $\phi\left(r_{1}\right), \phi\left(r_{2}\right) \in \mathcal{W}$, and thus, $s\left(r_{1}\right)=s\left(r_{2}\right)$, as desired.

Finally, to establish (C3) let $\mathbf{x}, \mathbf{y} \in \mathcal{X}$ with $d(\mathbf{x}, \mathbf{y}) \geq 3$, and let $\mathbf{b} \in{ }^{o} \mathcal{B}_{\mathbf{x}, \mathbf{y}}^{\phi}$ Then $\mathbf{b} \in{ }^{o} \mathcal{B}_{\mathbf{x}, \mathbf{y}}^{\psi}$ 
also, so

$$
\begin{aligned}
0 & =(\mathbf{x}-\mathbf{y}) \bullet \psi(\mathbf{b})=\sum_{k \in \mathcal{K}_{ \pm}(\mathbf{x}, \mathbf{y})}\left(x_{k}-y_{k}\right) \psi\left(b_{k}\right) \\
\overline{\overline{(*)}} & \sum_{k \in \mathcal{K}_{ \pm}(\mathbf{x}, \mathbf{y})}\left(x_{k}-y_{k}\right)\left(s\left(b_{k}\right) \phi\left(b_{k}\right)+t\left(b_{k}\right)\right) \\
\overline{\overline{(\dagger)}} & s \sum_{k \in \mathcal{K}_{ \pm}(\mathbf{x}, \mathbf{y})}\left(x_{k}-y_{k}\right)\left(\phi\left(b_{k}\right)+t\left(b_{k}\right)\right) \\
& =s(\mathbf{x}-\mathbf{y}) \bullet \phi(\mathbf{b})+\sum_{k \in \mathcal{K}_{ \pm}(\mathbf{x}, \mathbf{y})}\left(x_{k}-y_{k}\right) t\left(b_{k}\right) \\
\overline{\overline{(\dagger)}} & \sum_{k \in \mathcal{K}_{ \pm}(\mathbf{x}, \mathbf{y})}\left(x_{k}-y_{k}\right) t\left(b_{k}\right) .
\end{aligned}
$$

Here, $(*)$ is by $(\mathrm{C} 4),(\diamond)$ is because $(\mathrm{C} 1)$ yields some $s>0$ such that $s\left(b_{k}\right)=s$ for all $k \in \mathcal{K}_{ \pm}(\mathbf{x}, \mathbf{y})$, and $(\dagger)$ is because $\mathbf{b} \in{ }^{o} \mathcal{B}_{\mathbf{x}, \mathbf{y}}^{\phi}$.

Proof of Theorem 6.4. If $\phi$ and $\psi$ are continuous on $[-1,1]$, then $F_{\phi}$ and $F_{\psi}$ are upper hemicontinuous on $\Delta(\mathcal{X})$, by Proposition 3.6.

"£" If $\psi(r)=s \phi(r)$ for all $r \in \mathcal{R}_{\mathcal{X}}^{F}$, then $\psi$ is certainly an $\mathcal{X}$-compatible affine transform of $\phi$. Thus, Theorem E.7(a) says that $F_{\phi}(\mathcal{X}, \mu)=F_{\psi}(\mathcal{X}, \mu)$ for all $\mu \in \Delta(\mathcal{X})$.

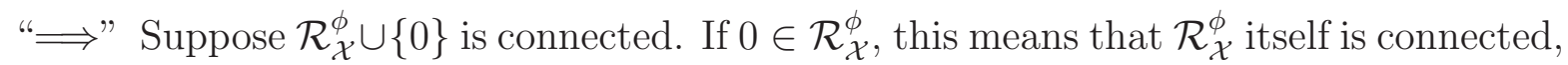
and hence, Lemma E.4(f) says it contains only one pivotal class.

Otherwise, $\mathcal{R}_{\mathcal{X}}^{\phi}$ has at most two connected components: $\mathcal{R}^{+}:=\mathcal{R}_{\mathcal{X}}^{\phi} \cap(0,1]$ and $\mathcal{R}^{-}:=$ $\mathcal{R}_{\mathcal{X}}^{\phi} \cap[-1,0)$. Lemma E.4(f) says that each of these contains at most one pivotal class, and since pivotal classes are invariant under negation, we again conclude that $\mathcal{R}_{\mathcal{X}}^{\phi}$ has only one pivotal class.

If $\phi$ and $\psi$ are continuous on $[-1,1]$, and $F_{\phi}(\mathcal{X}, \mu)=F_{\psi}(\mathcal{X}, \mu)$ for all $\mu \in \Delta(\mathcal{X})$, then Theorem E.7(b) says that $\psi$ is an $\mathcal{X}$-compatible affine transform of $\phi$. But $\mathcal{R}_{\mathcal{X}}^{F}$ contains only one pivotal class, so (C1) implies that $s$ is constant on $\mathcal{R}_{\mathcal{X}}^{\phi}$; thus, $\psi(r)=s \phi(r)+t(r)$ for all $r \in \mathcal{R}_{\mathcal{X}}^{F}$. Next, (C2) implies that the function $t(\bullet)$ takes a constant value on $t^{+}$ on $\mathcal{R}^{+}$. But then we have

$$
t^{+}=0+t^{+}=s(0)+t^{+} \overline{\overline{(*)}} \lim _{r \searrow 0} s \phi(r)+t^{+}=\lim _{r \searrow 0} \psi(r) \overline{\overline{(\dagger)}} 0 .
$$

Here, $(*)$ is because $\phi$ is continuous and odd, while $(\dagger)$ is because $\psi$ is continuous and odd.

We conclude that $t^{+}=0$. An identical argument, shows that $t=0$ on $\mathcal{R}^{-}$also. We conclude that $\psi(r)=s \phi(r)$ for all $r \in \mathcal{R}_{\mathcal{X}}^{F}$. 
Proof of Lemma 6.5 (a) Fix $\mathbf{x}, \mathbf{y} \in \mathcal{X}$, let $\mathbf{b} \in{ }^{o} \mathcal{B}_{\mathbf{x}, \mathbf{y}}^{F}$, and for any $s>0$, define $\mathbf{b}(s):=$ $\phi^{-1}(s \phi(\mathbf{b}))$. Then we have $\mathbf{z} \bullet \phi(\mathbf{b}(s))=s \mathbf{z} \bullet \phi(\mathbf{b})$ for all $\mathbf{z} \in \mathcal{X}$. Thus, for all $\mathbf{z} \in \mathcal{X} \backslash\{\mathbf{x}, \mathbf{y}\}$, we have $\mathbf{x} \bullet \phi(\mathbf{b}(s))=\mathbf{y} \bullet \phi(\mathbf{b}(s))>\mathbf{z} \bullet \phi(\mathbf{b}(s))$, because $\mathbf{x} \bullet \phi(\mathbf{b})=$ $\mathbf{y} \bullet \phi(\mathbf{b})>\mathbf{z} \bullet \phi(\mathbf{b})$, because $\mathbf{b} \in{ }^{\circ} \mathcal{B}_{\mathbf{x}, \mathbf{y}}^{F}$. Thus, if $\mathbf{b}(s) \in \mathcal{C}$, then $\mathbf{b}(s) \in{ }^{o} \mathcal{B}_{\mathbf{x}, \mathbf{y}}^{F}$ also.

But if $\phi(\mathcal{C})$ is star-shaped, then $s \phi(\mathbf{b}) \in \phi(\mathcal{C})$ for all $s \in[0,1]$. Thus, we have $\mathbf{b}(s) \in{ }^{o} \mathcal{B}_{\mathbf{x}, \mathbf{y}}^{F}$ for all $s \in(0,1]$. But this describes a continuous path from $\mathbf{b}$ to $\mathbf{0}$. Thus, for any $k \in \mathcal{K}_{ \pm}(\mathbf{x}, \mathbf{y})$, the map $s \mapsto b_{k}(s)$ describes a continuous path from $b_{k}$ to 0 .

This argument applies for any $\mathbf{x}, \mathbf{y} \in \mathcal{X}$, any $\mathbf{b} \in{ }^{o} \mathcal{B}_{\mathbf{x}, \mathbf{y}}^{F}$, and any $k \in \mathcal{K}_{ \pm}(\mathbf{x}, \mathbf{y})$. Thus, every element of $\mathcal{R}_{\mathcal{X}}^{\phi} \cup\{0\}$ is path-connected to 0 .

(b) The function $\phi^{d}(r)=\operatorname{sign}(r)|r|^{d}$ is homogeneous. Thus, the function $\phi^{d}: \mathbb{R}^{\mathcal{K}} \longrightarrow \mathbb{R}^{\mathcal{K}}$ defined by $\phi^{d}(\mathbf{r})=\left(\phi^{d}\left(r_{k}\right)\right)_{k \in \mathcal{K}}$ acts homogeneously on $\mathbb{R}^{\mathcal{K}}$. By hypothesis, $\mathbf{0} \in \mathcal{C}$, and $\mathcal{C}$ is convex, hence star-shaped. Thus, $\phi^{d}(\mathcal{C})$ is also star-shaped. Thus, if $\left\|\phi-\phi^{d}\right\|_{\infty}$ is sufficiently small, then $\phi(\mathcal{C})$ is also star-shaped. Thus, part (a) implies that $\mathcal{R}_{\mathcal{X}}^{\phi} \cup\{0\}$ is connected.

(c) Let $r \in \mathcal{R}_{\mathcal{X}}^{F}$. Suppose $r>0$; we will show that $(0, r] \subseteq \mathcal{R}_{\mathcal{X}}^{F}$. By definition, there exist $\mathbf{x}, \mathbf{y} \in \mathcal{X}$ with $d(\mathbf{x}, \mathbf{y}) \geq 3$ and $\mathbf{b} \in{ }^{\circ} \mathcal{B}_{\mathbf{x}, \mathbf{y}}^{F}$ and $k \in \mathcal{K}_{ \pm}(\mathbf{x}, \mathbf{y})$ such that $b_{k}=r$. Now, for all $s \in(0,1)$, let $\mathbf{b}^{s}:=s \mathbf{b}+(1-s)(\mathbf{x}+\mathbf{y}) / 2$. Then $\mathbf{b}^{s} \in{ }^{o} \mathcal{B}_{\mathbf{x}, \mathbf{y}}^{F}$ by neutral reinforcement, and $b_{k}^{s}=s b_{k}+(1-s)\left(x_{k}+y_{k}\right) / 2=s r+(1-s) 0=s r$. Thus, $s r \in \mathcal{R}_{\mathcal{X}}^{F}$.

Thus, $\mathcal{R}_{\mathcal{X}}^{F} \cup\{0\}$ is an interval; thus, it is connected.

\section{Proofs from $\S 5$}

Proof of Theorem 5.1. (a) Fix $d \in(0, \infty)$. Recall that we define $\phi^{d}(r):=\operatorname{sign}(r) \cdot|r|^{d}$, and $H^{d}:=F_{\phi^{d}}$. Proposition 3.6 says that $H^{d}$ is upper hemicontinuous, because $\phi^{d}$ is continuous on $[-1,1]$. Clearly, $\phi^{d}$ is regular. It remains to show that $H^{d}$ satisfies neutral reinforcement.

To see this, let $\mathbf{x}, \mathbf{y} \in \mathcal{X}$, and let $\mathbf{b} \in \mathcal{B}_{\mathbf{x}, \mathbf{y}}^{\phi^{d}}$. Let $s \in[0,1]$, and let $\mathbf{b}^{s}:=s \mathbf{b}+(1-s)(\mathbf{x}+$ $\mathbf{y}) / 2$. We must show that $\mathbf{b}^{s} \in \mathcal{B}_{\mathbf{x}, \mathbf{y}}^{\phi^{d}}$. To see this, note that

$$
\begin{aligned}
(\mathbf{x}-\mathbf{y}) \bullet \phi^{d}\left(\mathbf{b}^{s}\right) & =\sum_{k \in \mathcal{K}_{ \pm}(\mathbf{x}, \mathbf{y})}\left(x_{k}-y_{k}\right) \cdot \phi^{d}\left(b_{k}^{s}\right) \quad \overline{\overline{(*)}} \sum_{k \in \mathcal{K}_{ \pm}(\mathbf{x}, \mathbf{y})}\left(x_{k}-y_{k}\right) \cdot \phi^{d}\left(s b_{k}\right) \\
& =\sum_{k \in \mathcal{K}_{ \pm}(\mathbf{x}, \mathbf{y})}\left(x_{k}-y_{k}\right) \cdot s^{d} \phi^{d}\left(b_{k}\right)=s^{d} \sum_{k \in \mathcal{K}_{ \pm}(\mathbf{x}, \mathbf{y})}\left(x_{k}-y_{k}\right) \cdot \phi^{d}\left(b_{k}\right) \\
& =s^{d} \cdot(\mathbf{x}-\mathbf{y}) \bullet \phi^{d}(\mathbf{b}) \overline{\overline{(\dagger)}} 0 .
\end{aligned}
$$

Here, $(\dagger)$ is because $\mathbf{b} \in \mathcal{B}_{\mathbf{x}, \mathbf{y}}^{\phi^{d}}$, while $(*)$ is because for all $k \in \mathcal{K}_{ \pm}(\mathbf{x}, \mathbf{y})$, we have $\left(x_{k}+\right.$ $\left.y_{k}\right) / 2=0$, and thus, $b_{k}^{s}=s b_{k}+(1-s)\left(x_{k}+y_{k}\right) / 2=s b_{k}$. 
Meanwhile, for any other $\mathbf{z} \in \mathcal{X}$, we have

$$
\begin{aligned}
(\mathbf{x}-\mathbf{z}) \bullet \phi^{d}\left(\mathbf{b}^{s}\right) & =\sum_{k \in \mathcal{K}}\left(x_{k}-z_{k}\right) \cdot \phi^{d}\left(b_{k}^{s}\right) \\
& =\sum_{k \in \mathcal{K}_{ \pm}(\mathbf{x}, \mathbf{y})}\left(x_{k}-z_{k}\right) \cdot \phi^{d}\left(b_{k}^{s}\right)+\sum_{k \in \mathcal{K}_{0}(\mathbf{x}, \mathbf{y})}\left(x_{k}-z_{k}\right) \cdot \phi^{d}\left(b_{k}^{s}\right) \\
& =s^{d} \sum_{k \in \mathcal{K}_{ \pm}(\mathbf{x}, \mathbf{y})}\left(x_{k}-z_{k}\right) \cdot \phi^{d}\left(b_{k}\right)+\sum_{k \in \mathcal{K}_{0}(\mathbf{x}, \mathbf{y})}\left(x_{k}-z_{k}\right) \cdot \phi^{d}\left(b_{k}^{s}\right) \\
& \underset{(\dagger)}{\geq} s^{d} \sum_{k \in \mathcal{K}_{ \pm}(\mathbf{x}, \mathbf{y})}\left(x_{k}-z_{k}\right) \cdot \phi^{d}\left(b_{k}\right)+s^{d} \sum_{k \in \mathcal{K}_{0}(\mathbf{x}, \mathbf{y})}\left(x_{k}-z_{k}\right) \cdot \phi^{d}\left(b_{k}\right) \\
& =s^{d} \cdot(\mathbf{x}-\mathbf{z}) \bullet \phi^{d}(\mathbf{b}) \underset{(\diamond)}{\geq} 0 .
\end{aligned}
$$

Here $(*)$ is as in eqn.(E16), and $(\diamond)$ is because $\mathbf{x} \in F_{\phi^{d}}(\mathbf{b})$ because $\mathbf{b} \in{ }^{o} \mathcal{B}_{\mathbf{x}, \mathbf{y}}^{\phi^{d}}$. To see $(\dagger)$, let $k \in \mathcal{K}_{0}(\mathbf{x}, \mathbf{y})$. If $x_{k}=y_{k}=1$, then $b_{k}^{s}=s b_{k}+(1-s)>s b_{k}$, so $\phi^{d}\left(b_{k}^{s}\right)>\phi^{d}\left(s b_{k}\right)=$ $s^{d} \phi^{d}\left(b_{k}\right)$. Meanwhile, $\left(x_{k}-z_{k}\right) \geq 0$, so $\left(x_{k}-z_{k}\right) \cdot \phi^{d}\left(b_{k}^{s}\right) \geq\left(x_{k}-z_{k}\right) \cdot s^{d} \phi^{d}\left(b_{k}\right)$.

If $x_{k}=y_{k}=-1$, then $b_{k}^{s}=s b_{k}-(1-s)<s b_{k}$, so $\phi^{d}\left(b_{k}^{s}\right)<\phi^{d}\left(s b_{k}\right)=s^{d} \phi^{d}\left(b_{k}\right)$. But now $\left(x_{k}-z_{k}\right) \leq 0$, so again $\left(x_{k}-z_{k}\right) \cdot \phi^{d}\left(b_{k}^{s}\right) \geq\left(x_{k}-z_{k}\right) \cdot s^{d} \phi^{d}\left(b_{k}\right)$.

We conclude that $\mathbf{x} \bullet \phi^{d}\left(\mathbf{b}^{s}\right)=\mathbf{y} \bullet \phi^{d}\left(\mathbf{b}^{s}\right) \geq \mathbf{z} \bullet \phi^{d}\left(\mathbf{b}^{s}\right)$ for all $\mathbf{z} \in \mathcal{X}$; thus, $\mathbf{b}^{s} \in \mathcal{B}_{\mathbf{x}, \mathbf{y}}^{\phi^{d}}$.

Theorem 5.1(b) "£" is just a special case of Theorem 5.1(a). The proof of Theorem

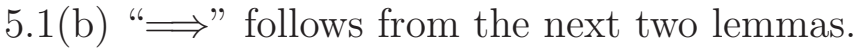

Lemma E.9 Let $\mathcal{X}$ be a thick judgement space. If $F: \Delta(\mathcal{X}) \rightrightarrows \mathcal{X}$ is regular, upper hemicontinuous and satisfies neutral reinforcement, then $F=F_{\psi}$ for some continuous gain function $\psi:[-1,1] \longrightarrow \mathbb{R}$.

Proof: If $\mathcal{X}$ is proximal, then Theorem 2.1 says that $\mathcal{X}$ is supermajoritarian determinate, so any additive rule is identical to the median rule. Thus, $F=F_{\phi}$ where $\phi(r)=r$ for all $r \in[-1,1]$. So, assume $\mathcal{X}$ is not proximal.

Let $\phi:[-1,1] \longrightarrow{ }^{*} \mathbb{R}$ be any odd, regular gain function such that $F=F_{\phi}$. Then Lemma 6.1 says that $\mathcal{R}_{\mathcal{X}}^{F} \neq \emptyset$. Thus, Proposition 6.5(c) says that there is some $R>0$ such that either $\mathcal{R}_{\mathcal{X}}^{\phi}=(-R, R)$, or $\mathcal{R}_{\mathcal{X}}^{\phi}=(-R, 0) \sqcup(0, R)$. Fix $r_{1}<r_{2} \in(0, R)$, and for all $r \in[-1,1]$, define

$$
\hat{\phi}(r) \quad:=\operatorname{st}\left(\frac{\phi(r)}{\phi\left(r_{2}\right)-\phi\left(r_{1}\right)}\right) \quad \text { and } \quad \varphi_{0} \quad:=\lim _{r \searrow 0} \hat{\phi}(r) .
$$

Since $\phi$ is regular, we can choose $r_{1}, r_{2}$ such that $\hat{\phi}(r)$ is finite for some $r \in \mathcal{R}$. Thus, $\hat{\phi}(r)$ is finite for all $r$ close to zero.

For any $\mathbf{r} \in \mathbb{R}^{\mathcal{K}}$, let $\operatorname{sign}(\mathbf{r}):=\left(\operatorname{sign}\left(r_{k}\right)\right)_{k \in \mathcal{K}} \in\{-1,0,1\}^{\mathcal{K}}$. We will say that $F_{\phi}$ satisfies parity on $\mathcal{X}$ if, for all $\mathbf{x}, \mathbf{y} \in \mathcal{X}$, and all $\mathbf{b} \in{ }^{o} \mathcal{B}_{\mathbf{x}, \mathbf{y}}^{\phi}$, we have $\operatorname{sign}(\mathbf{b}) \bullet(\mathbf{x}-\mathbf{y})=0$

Claim 1: Either $\varphi_{0}=0$, or $F_{\phi}$ satisfies parity. 
Proof: (by contradiction) Suppose $\varphi_{0}>0$. Then there is some $\epsilon>0$ such that $\varphi_{0} \leq \hat{\phi}(r)<\left(1+\frac{1}{K}\right) \varphi_{0}$ for all $r \in(0, \epsilon)$. Dividing both sides by $\varphi_{0}$ and using the fact that $\hat{\phi}$ is odd, we conclude that

$$
1 \leq \frac{|\hat{\phi}(r)|}{\varphi_{0}}<1+\frac{1}{K}, \quad \text { for all } r \in[-1,1] \text { with } 0<|r|<\epsilon .
$$

Suppose also that $F_{\phi}$ does not satisfy parity. Then there exist some $\mathbf{x}, \mathbf{y} \in \mathcal{X}$ and $\mathbf{b} \in{ }^{\circ} \mathcal{B}_{\mathbf{x}, \mathbf{y}}^{\phi}$ such that $\operatorname{sign}(\mathbf{b}) \bullet(\mathbf{x}-\mathbf{y})=c \neq 0$. Observe that $c \in 2 \mathbb{Z}$, because $\operatorname{sign}(\mathbf{b}) \in \mathbb{Z}^{\mathcal{K}}$ and $(\mathbf{x}-\mathbf{y}) \in 2 \mathbb{Z}^{\mathcal{K}}$. Thus, without loss of generality (by switching $\mathbf{x}$ and $\mathbf{y}$ if necessary) we can assume that $c \geq 2$. Find $\mu \in \Delta(\mathcal{X})$ such that $\widetilde{\mu}=\mathbf{b}$. Recall that $\delta^{\mathbf{x}, \mathbf{y}} \in \Delta(\mathcal{X})$ is the profile such that $\delta^{\mathbf{x}, \mathbf{y}}(\mathbf{x})=\frac{1}{2}=\delta^{\mathbf{x}, \mathbf{y}}(\mathbf{y})$ (thus, $\widetilde{\delta}^{\mathbf{x}, \mathbf{y}}=\frac{1}{2}(\mathbf{x}+\mathbf{y})$ ). Define $\nu:=(1-\epsilon) \delta^{\mathbf{x}, \mathbf{y}}+\epsilon \mu$.

Claim 1.1: For all $k \in \mathcal{K}_{ \pm}(\mathbf{x}, \mathbf{y})$, we have $\left|\hat{\phi}\left(\widetilde{\nu}_{k}\right) / \varphi_{0}-\operatorname{sign}\left(\widetilde{\mu}_{k}\right)\right|<\frac{c}{2 K}$.

Proof: If $k \in \mathcal{K}_{ \pm}(\mathbf{x}, \mathbf{y})$, then $\widetilde{\delta}_{k}^{\mathbf{x}, \mathbf{y}}=0$, so $\left|\widetilde{\nu}_{k}\right|=\epsilon\left|\widetilde{\mu}_{k}\right|<\epsilon$, and thus, $1 \leq\left|\hat{\phi}\left(\widetilde{\nu}_{k}\right)\right| / \varphi_{0}<$ $1+\frac{1}{K}$, by statement (E18). In other words, $\left|\hat{\phi}\left(\widetilde{\nu}_{k}\right) / \varphi_{0}-\operatorname{sign}\left(\hat{\phi}\left(\widetilde{\nu}_{k}\right)\right)\right|<\frac{1}{K} \leq \frac{c}{2 K}$, where the last inequality is because $c \geq 2$. But $\operatorname{sign}\left(\hat{\phi}\left(\widetilde{\nu}_{k}\right)\right)=\operatorname{sign}\left(\widetilde{\nu}_{k}\right)=\operatorname{sign}\left(\epsilon \widetilde{\mu}_{k}\right)=$ $\operatorname{sign}\left(\widetilde{\mu}_{k}\right)$. The claim follows.

$\nabla$ Claim 1.1

Thus,

$$
\begin{aligned}
& \left|(\mathbf{x}-\mathbf{y}) \bullet\left(\hat{\phi}(\widetilde{\nu}) / \varphi_{0}-\operatorname{sign}(\widetilde{\mu})\right)\right|=\left|\sum_{k \in \mathcal{K}_{ \pm}(\mathbf{x}, \mathbf{y})}\left(x_{k}-y_{k}\right)\left(\hat{\phi}\left(\widetilde{\nu}_{k}\right) / \varphi_{0}-\operatorname{sign}\left(\widetilde{\mu}_{k}\right)\right)\right| \\
& \leq \sum_{k \in \mathcal{K}_{ \pm}(\mathbf{x}, \mathbf{y})}\left|\hat{\phi}\left(\widetilde{\nu}_{k}\right) / \varphi_{0}-\operatorname{sign}\left(\widetilde{\mu}_{k}\right)\right| \\
& \leq \sum_{(*)} \frac{c}{2 K} \leq \frac{c}{2},
\end{aligned}
$$

where $(*)$ is by Claim 1.1. Thus,

$$
\begin{aligned}
\text { st } & \left(\frac{(\mathbf{x}-\mathbf{y}) \bullet \phi(\widetilde{\nu})}{\varphi_{0} \cdot\left(\phi\left(r_{2}\right)-\phi\left(r_{1}\right)\right)}\right) \quad \overline{\overline{(\dagger)}} \frac{1}{\varphi_{0}}(\mathbf{x}-\mathbf{y}) \bullet \operatorname{st}\left(\frac{\phi(\widetilde{\nu})}{\phi\left(r_{2}\right)-\phi\left(r_{1}\right)}\right) \\
& \overline{\overline{(\diamond)}} \frac{1}{\varphi_{0}}(\mathbf{x}-\mathbf{y}) \bullet \hat{\phi}(\widetilde{\nu}) \\
& =(\mathbf{x}-\mathbf{y}) \bullet \operatorname{sign}(\widetilde{\mu})+(\mathbf{x}-\mathbf{y}) \bullet\left(\hat{\phi}(\widetilde{\nu}) / \varphi_{0}-\operatorname{sign}(\widetilde{\mu})\right) \\
& =c+(\mathbf{x}-\mathbf{y}) \bullet\left(\hat{\phi}(\widetilde{\nu}) / \varphi_{0}-\operatorname{sign}(\widetilde{\mu})\right) \underset{(*)}{\geq} c-\frac{c}{2}=\frac{c}{2}>
\end{aligned}
$$

Here, $(\dagger)$ is because the map st : ${ }^{*} \mathbb{R} \longrightarrow \mathbb{R}$ is a ring homomorphism, and $\varphi_{0} \in \mathbb{R}$, and $(\mathbf{x}-\mathbf{y}) \in \mathbb{R}^{\mathcal{K}}$, so st maps these objects to themselves. Meanwhile, $(\diamond)$ is by definition (E17), and $(*)$ is by inequality (E19).

But $\varphi_{0} \cdot\left(\phi\left(r_{2}\right)-\phi\left(r_{1}\right)\right)>0$. Thus, inequality $($ E20) implies that st $((\mathbf{x}-\mathbf{y}) \bullet \phi(\widetilde{\nu}))>0$; thus $(\mathbf{x}-\mathbf{y}) \bullet \phi(\widetilde{\nu})>0$, and thus, $\mathbf{y} \notin F_{\phi}(\nu)$. But this contradicts neutral reinforcement. 
Now, fix $S \in(0, R)$, and define $\psi:[-1,1] \longrightarrow \mathbb{R}$ by setting $\psi(0)=0$ and $\psi(r):=$ $\hat{\phi}(S r)-\varphi_{0}$ for all $r \in(0,1]$, and $\psi(r):=\hat{\phi}(S r)+\varphi_{0}$ for all $r \in[-1,0)$.

Claim 2: $\quad \psi$ is odd, real-valued, continuous, and increasing on $[-1,1]$.

Proof: $\psi$ is odd because $\hat{\phi}$ is odd because $\phi$ is odd. Now, let $\bar{\phi}$ be as defined in Theorem $6.2(\mathrm{a})$. Then for all $r \in(0,1]$, we have

$$
\begin{aligned}
\psi(r) & =\hat{\phi}(S r)-\varphi_{0}=\hat{\phi}(S r)-\hat{\phi}\left(r_{1}\right)+\hat{\phi}\left(r_{1}\right)-\varphi_{0} \\
& \overline{(*)} \operatorname{st}\left(\frac{\phi(S r)-\phi\left(r_{1}\right)}{\phi\left(r_{2}\right)-\phi\left(r_{1}\right)}\right)+\left(\hat{\phi}\left(r_{1}\right)-\varphi_{0}\right) \\
& =\bar{\phi}(S r)+(\text { a real constant })
\end{aligned}
$$

where $(*)$ is by substituting in definition (E17), and recalling that st : * $\mathbb{R} \longrightarrow \mathbb{R}$ is a ring homomorphism. But Theorem 6.2(a) says that $\bar{\phi}$ is real-valued, continuous, and increasing on $\mathcal{R}$, which in particular means it has these properties on $(0, R)$. Since $S<R$, it follows that $\psi$ is real-valued, continuous, and increasing on $(0,1)$. Since $\psi$ is odd, it is also real-valued, continuous, and increasing on $(-1,0)$. Finally, by construction we have

$$
\lim _{r \searrow 0} \psi(r)=\lim _{r \searrow 0} \hat{\phi}(r)-\varphi_{0} \quad \overline{\overline{(\diamond)}} \varphi_{0}-\varphi_{0}=0
$$

where $(\diamond)$ is by definition (E17), Thus, $\psi$ is right-continuous at 0 . Since $\psi$ is odd, it is also left-continuous at 0 .

$\diamond$ Claim 2

It remains to show that $F_{\psi}=F_{\phi}$. The gain function $\phi$ is increasing by definition, while $\psi$ is increasing by Claim 2; thus, both $F_{\phi}$ and $F_{\psi}$ are monotone by Proposition 3.5. Furthermore, $\psi$ is continuous by Claim 2, so $F_{\psi}$ is upper hemicontinuous by Proposition 3.6. Meanwhile, $F_{\phi}$ is upper hemicontinuous by hypothesis. Thus, we can apply Proposition C.3.

Claim 3: $\quad$ For any $\mathbf{x}, \mathbf{y} \in \mathcal{X}$, we have ${ }^{\circ} \mathcal{B}_{\mathbf{x}, \mathbf{y}}^{\phi} \cap \mathcal{C}_{\mathbf{x}}^{\psi} \subseteq \mathcal{C}_{\mathbf{y}}^{\psi}$.

Proof: Let $\mathbf{b} \in{ }^{o} \mathcal{B}_{\mathbf{x}, \mathbf{y}}^{\phi} \cap \mathcal{C}_{\mathbf{x}}^{\psi}$. We must show that $\mathbf{y} \in F_{\psi}(\mathbf{b})$. Since $\mathbf{x} \in F_{\psi}(\mathbf{b})$, it suffices to show that $\psi(\mathbf{b}) \bullet(\mathbf{x}-\mathbf{y})=0$.

Recall that $\widetilde{\delta}^{\mathbf{x}, \mathbf{y}}:=(\mathbf{x}+\mathbf{y}) / 2$. Thus,

$$
\begin{aligned}
\phi(S \mathbf{b}) \bullet(\mathbf{x}-\mathbf{y}) & =\sum_{k \in \mathcal{K}_{ \pm}(\mathbf{x}, \mathbf{y})} \phi\left(S b_{k}\right) \cdot\left(x_{k}-y_{k}\right) \\
& \overline{\overline{(*)}} \sum_{k \in \mathcal{K}_{ \pm}(\mathbf{x}, \mathbf{y})} \phi\left(S b_{k}+(1-S) \widetilde{\delta}_{k}^{\mathbf{x}, \mathbf{y}}\right) \cdot\left(x_{k}-y_{k}\right) \\
& =\phi\left(S \mathbf{b}+(1-S) \widetilde{\delta}^{\mathbf{x}, \mathbf{y}}\right) \bullet(\mathbf{x}-\mathbf{y}) \overline{\overline{(\dagger)}} 0 .
\end{aligned}
$$

Here $(*)$ is because $\widetilde{\delta}_{k}^{\mathbf{x}, \mathbf{y}}=0$ for all $k \in \mathcal{K}_{ \pm}(\mathbf{x}, \mathbf{y})$, and $(\dagger)$ is because $F_{\phi}(S \mathbf{b}+(1-$ 
$\left.S) \widetilde{\delta^{\mathbf{x}, \mathbf{y}}}\right)=\{\mathbf{x}, \mathbf{y}\}$, by neutral reinforcement. Finally, observe that

$$
\begin{aligned}
\psi(\mathbf{b}) & =\hat{\phi}(S \mathbf{b})-\varphi_{0} \cdot \operatorname{sign}(\mathbf{b}) . \quad \text { Thus, } \\
\psi(\mathbf{b}) \bullet(\mathbf{x}-\mathbf{y}) & =\hat{\phi}(S \mathbf{b}) \bullet(\mathbf{x}-\mathbf{y})-\varphi_{0} \cdot \operatorname{sign}(\mathbf{b}) \bullet(\mathbf{x}-\mathbf{y}) \\
& \overline{\overline{(*)}} \hat{\phi}(S \mathbf{b}) \bullet(\mathbf{x}-\mathbf{y}) \overline{\overline{(\ddagger)}} \operatorname{st}\left(\frac{\phi(S \mathbf{b})}{\phi\left(r_{2}\right)-\phi\left(r_{1}\right)}\right) \bullet \operatorname{st}(\mathbf{x}-\mathbf{y}) \\
& \overline{\overline{(\diamond)}} \operatorname{st}\left(\frac{\phi(S \mathbf{b}) \bullet(\mathbf{x}-\mathbf{y})}{\phi\left(r_{2}\right)-\phi\left(r_{1}\right)}\right) \quad \overline{\overline{(\dagger)}} \operatorname{st}(0)=0 .
\end{aligned}
$$

here, $(*)$ is because Claim 1 asserts that either $\varphi_{0}=0$ or $\operatorname{sign}(\mathbf{b}) \bullet(\mathbf{x}-\mathbf{y})=0$. Next, $(\ddagger)$ is by definition (E17), while $(\diamond)$ is because the map st : ${ }^{*} \mathbb{R} \longrightarrow \mathbb{R}$ is a ring homomorphism. Finally, $(\dagger)$ is by eqn. $(\mathrm{E} 21)$.

Thus, $\psi(\mathbf{b}) \bullet \mathbf{y}=\psi(\mathbf{b}) \bullet \mathbf{x}$. Thus, $\mathbf{y} \in F_{\psi}(\mathbf{b})$, because $\mathbf{x} \in F_{\psi}(\mathbf{b})$. This holds for all $\mathbf{b} \in{ }^{o} \mathcal{B}_{\mathbf{x}, \mathbf{y}}^{\phi} \cap \mathcal{C}_{\mathbf{x}}^{\psi}$. Thus, ${ }^{o} \mathcal{B}_{\mathbf{x}, \mathbf{y}}^{\phi} \cap \mathcal{C}_{\mathbf{x}}^{\psi} \subseteq \mathcal{C}_{\mathbf{y}}^{\psi}$.

$\diamond$ Claim 3

Combining Proposition C.3 and Claim 2 yields $F_{\psi}=F_{\phi}$.

Lemma E.10 Let $\mathcal{X}$ be a thick judgement space, and let $\phi:[-1,1] \longrightarrow \mathbb{R}$ be a continuous gain function. If $F_{\phi}$ satisfies neutral reinforcement on $\mathcal{X}$, then $F_{\phi}=H^{d}$ for some $d \in$ $(0, \infty)$

Proof:

Claim 1: Fix $s \in(0,1)$. Define $\psi:[-1,1] \longrightarrow \mathbb{R}$ by $\psi(r):=\phi(s r)$. Then $F_{\psi}=F_{\phi}$.

Proof: We will use Proposition C.3. Let $\mathbf{x}, \mathbf{y} \in \mathcal{X}$, and let $\mathbf{b} \in{ }^{\circ} \mathcal{B}_{\mathbf{x}, \mathbf{y}}^{\phi}$. We must show that $\mathbf{b} \in \mathcal{B}_{\mathbf{x}, \mathbf{y}}^{\psi} \cup\left(\operatorname{conv}(\mathcal{X}) \backslash \mathcal{C}_{\mathbf{x}}^{\psi}\right)$.

To see this, let $\mathbf{b}^{s}:=s \mathbf{b}+(1-s)(\mathbf{x}+\mathbf{y}) / 2$. Then

$$
\begin{aligned}
(\mathbf{x}-\mathbf{y}) \bullet \psi(\mathbf{b}) & =\sum_{k \in \mathcal{K}_{ \pm}(\mathbf{x}, \mathbf{y})}\left(x_{k}-y_{k}\right) \cdot \psi\left(b_{k}\right)=\sum_{k \in \mathcal{K}_{ \pm}(\mathbf{x}, \mathbf{y})}\left(x_{k}-y_{k}\right) \cdot \phi\left(s b_{k}\right) \\
\overline{\overline{(*)}} & \sum_{k \in \mathcal{K}_{ \pm}(\mathbf{x}, \mathbf{y})}\left(x_{k}-y_{k}\right) \cdot \phi\left(b_{k}^{s}\right)=(\mathbf{x}-\mathbf{y}) \bullet \phi\left(\mathbf{b}^{s}\right) \overline{\overline{(\dagger)}} 0 .
\end{aligned}
$$

Here, $(*)$ is because for all $k \in \mathcal{K}_{ \pm}(\mathbf{x}, \mathbf{y})$, we have $\left(x_{k}+y_{k}\right) / 2=0$, and thus, $b_{k}^{s}=$ $s b_{k}+(1-s)\left(x_{k}+y_{k}\right) / 2=s b_{k}$. Next, $(\dagger)$ is because $\mathbf{b}^{s} \in \mathcal{B}_{\mathbf{x}, \mathbf{y}}^{\phi}$, because $\mathbf{b} \in{ }^{o} \mathcal{B}_{\mathbf{x}, \mathbf{y}}^{\phi}$ and $F_{\phi}$ satisfies neutral reinforcement.

Thus, $\mathbf{x} \bullet \psi(\mathbf{b})=\mathbf{y} \bullet \psi(\mathbf{b})$. If $\mathbf{x} \bullet \psi(\mathbf{b}) \geq \mathbf{z} \bullet \psi(\mathbf{b})$ for all $\mathbf{z} \in \mathcal{X}$, then $\mathbf{b} \in \mathcal{B}_{\mathbf{x}, \mathbf{y}}^{\psi}$; otherwise, $\mathbf{b} \in \operatorname{conv}(\mathcal{X}) \backslash \mathcal{C}_{\mathbf{x}}^{G}$.

$\diamond$ Claim 1

Claim 2: $\quad$ There exists a continuous, increasing function $\sigma:(0,1) \longrightarrow \mathbb{R}$ such that, for all $r \in \mathcal{R}_{\mathcal{X}}^{\phi}$ and $s \in(0,1)$, we have $\phi(s r)=\sigma(s) \cdot \phi(r)$. 
Proof: Proposition 6.5(c) implies that $\mathcal{R}_{\mathcal{X}}^{\phi} \cup\{0\}$ is connected. Fix $s \in(0,1)$ and define $\psi$ as in Claim 1. Then Claim 1 and Theorem 6.4 imply that there is some scalar $\sigma(s)>0$ such that $\psi(r)=\sigma(s) \cdot \phi(r)$ for all $r \in \mathcal{R}_{\mathcal{X}}^{\phi}$.

Finally, $\sigma$ continuous and increasing because $\phi$ continuous and increasing. $\diamond$ claim 2

Claim 3: For all $s, t \in(0,1]$, we have $\sigma(s t)=\sigma(s) \cdot \sigma(t)$.

Proof: Fix nonzero $r \in \mathcal{R}_{\mathcal{X}}^{\phi}$. Proposition 6.5(c) says $t r \in \mathcal{R}_{\mathcal{X}}^{\phi}$, because $t \in(0,1]$. Thus,

$$
\sigma(s t) \cdot \phi(r)=\phi(s t r)=\sigma(s) \cdot \phi(t r)=\sigma(s) \cdot \sigma(t) \cdot \phi(r)
$$

where every equality is by Claim 2. Now divide both sides by $\phi(r)$. (Note that $\phi(r) \neq 0$ because $r \neq 0$ and $\phi$ is strictly increasing, while $\phi(0)=0$ because $\phi$ is odd.) $\diamond$ claim 3

Claim 4: $\quad$ There is some $d>0$ such that $\sigma(s)=s^{d}$ for all $d \in[0,1]$.

Proof: Define $\lambda(s):=\log \left(\sigma\left(e^{-s}\right)\right)$ for $s \in(0, \infty)$. Then $\lambda$ is continuous and decreasing on $(0, \infty)$ because $\sigma$ continuous and increasing on $(0,1)$. However, Claim 3 implies that $\lambda$ satisfies the Cauchy functional equation: $\lambda(s+t)=\lambda(s)+\lambda(t)$ for all $s, t \in(0, \infty)$. Thus, there exists some $d>0$ such that $\lambda(s)=d s$ for all $s \in(0, \infty)$. But this means that $\sigma(s)=s^{d}$ for all $s \in(0,1)$.

$\diamond$ Claim 4

Now fix $R \in \mathcal{R}_{\mathcal{X}}^{\phi}$. Let $C:=\phi(R) / R^{d}$. For all $r \in[0, R]$, we have

$$
\phi(r)=\phi((r / R) \cdot R) \overline{\overline{(\diamond)}} \sigma(r / R) \cdot \phi(R) \overline{\overline{(*)}}(r / R)^{d} \cdot \phi(R)=C \cdot r^{d},
$$

where $(\diamond)$ is by Claim 2 , and $(*)$ is by Claim 4 .

Since $R$ is arbitrary, we conclude that there is some constant $C>0$ such that $\phi(r)=C \cdot r^{d}$ for all positive $r \in \mathcal{R}_{\mathcal{X}}^{\phi}$. Since $\phi$ is odd, this implies that $\phi(r)=C \cdot \phi^{d}(r)$ for all $r \in \mathcal{R}_{\mathcal{X}}^{\phi}$. But then Theorem 6.4 implies that $F_{\phi}=H^{d}$.

\section{Proofs from $\S 4$}

Lemma E.11 Let $d \in(0, \infty)$. If $H^{d}$ satisfies reinforcement on $\mathcal{X}$, then $H^{d}$ is the median rule on $\mathcal{X}$.

Proof: If $\mathcal{X}$ is proximal, then Theorem 2.1(a) says $\mathcal{X}$ is supermajoritarian determinate; thus, any SME additive rule on $\Delta(\mathcal{X})$ acts as the median rule.

Now suppose $\mathcal{X}$ is not proximal. Then there exist $\mathbf{x}, \mathbf{y} \in \mathcal{X}$ with $d(\mathbf{x}, \mathbf{y}) \geq 3$ and ${ }^{o} \mathcal{B}_{\mathbf{x}, \mathbf{y}}^{\phi^{d}} \neq \emptyset$. Let $\mathbf{b}^{0} \in{ }^{o} \mathcal{B}_{\mathbf{x}, \mathbf{y}}^{\phi^{d}}$, and find $i, j \in \mathcal{K}_{ \pm}(\mathbf{x}, \mathbf{y})$ such that $b_{i}^{0} \neq \pm b_{j}^{0}$. Lemma E.4(c) yields some other point $\mathbf{b}^{1} \in{ }^{o} \mathcal{B}_{\mathbf{x}, \mathbf{y}}^{\phi^{d}}$ such that $\mathbf{b}^{0}$ and $\mathbf{b}^{1}$ differ only in coordinates $i$ and $j$. Without loss of generality, suppose $x_{i}=1=-y_{i}$ and $y_{j}=1=-x_{j}$. 
For all $s \in[0,1]$, let $\mathbf{b}^{s}:=s \mathbf{b}^{1}+(1-s) \mathbf{b}^{0}$. Then $\mathbf{b}^{s} \in \mathcal{B}_{\mathbf{x}, \mathbf{y}}^{\phi^{d}}$ by reinforcement.

$$
\begin{aligned}
\text { Let } C & :=\sum_{k \in \mathcal{K}_{ \pm}(\mathbf{x}, \mathbf{y}) \backslash\{i, j\}}\left(x_{k}-y_{k}\right) \cdot \phi^{d}\left(b_{k}^{0}\right) . \\
\text { Then } 0 & =(\mathbf{x}-\mathbf{y}) \bullet \phi\left(\mathbf{b}^{s}\right)=\sum_{k \in \mathcal{K}_{ \pm}(\mathbf{x}, \mathbf{y})}\left(x_{k}-y_{k}\right) \cdot \phi^{d}\left(b_{k}^{s}\right) \\
& =\left(x_{i}-y_{i}\right) \phi^{d}\left(b_{i}^{s}\right)+\left(x_{j}-y_{j}\right) \phi^{d}\left(b_{j}^{s}\right)+C \\
& =2 \phi^{d}\left(b_{i}^{s}\right)-2 \phi^{d}\left(b_{j}^{s}\right)+C .
\end{aligned}
$$

(Note that $C$ is a constant which is independent of s.) To show that $H^{d}$ is the median rule, we must show that $d=1$. We will assume that $d \neq 1$, and derive a contradiction.

Let $b_{i}^{\prime}:=b_{i}^{1}-b_{i}^{0}$ and $b_{j}^{\prime}:=b_{j}^{1}-b_{j}^{0}$; then $b_{i}^{s}=b_{i}^{0}+s b_{i}^{\prime}$ and $b_{j}^{s}=b_{j}^{0}+s b_{j}^{\prime}$. Suppose that $b_{i}^{s}, b_{j}^{s} \geq 0$. (The argument for the cases $b_{i}^{s}, b_{j}^{s} \leq 0$ or $b_{i}^{s} \leq 0 \leq b_{j}^{s}$ are similar.) Then $\phi^{d}\left(b_{i}^{s}\right)=\left(b_{i}^{s}\right)^{d}$ and $\phi^{d}\left(b_{j}^{s}\right)=\left(b_{j}^{s}\right)^{d}$. Thus, $\left(\phi^{d}\right)^{\prime}\left(b_{i}^{s}\right)=d\left(b_{i}^{s}\right)^{d-1}$ and $\phi^{d}\left(b_{j}^{s}\right)=d\left(b_{j}^{s}\right)^{d-1}$. Differentiating equation (E23) with respect to $s$, we have

$$
\begin{aligned}
& 0=2 b_{i}^{\prime} \phi_{d}^{\prime}\left(b_{i}^{s}\right)-2 b_{j}^{\prime}\left(\phi_{d}\right)^{\prime}\left(b_{j}^{s}\right)=2 b_{i}^{\prime} d\left(b_{i}^{s}\right)^{d-1}-2 b_{j}^{\prime} d\left(b_{j}^{s}\right)^{d-1} \\
& \text { Thus, } \quad \frac{b_{j}^{\prime}}{b_{i}^{\prime}}=\left(\frac{b_{i}^{s}}{b_{j}^{s}}\right)^{d-1} . \\
& \text { Thus, if } d \neq 1, \text { then }\left(\frac{b_{j}^{\prime}}{b_{i}^{\prime}}\right)^{\frac{1}{d-1}}=\frac{b_{i}^{s}}{b_{j}^{s}}, \quad \text { for all } s \in[0,1] .
\end{aligned}
$$

Setting $s=0$ and $s=1$ in equation (E24), we get

$$
b_{i}^{0}=\left(\frac{b_{j}^{\prime}}{b_{i}^{\prime}}\right)^{\frac{1}{d-1}} b_{j}^{0} \quad \text { and } \quad b_{i}^{1}=\left(\frac{b_{j}^{\prime}}{b_{i}^{\prime}}\right)^{\frac{1}{d-1}} b_{j}^{1} .
$$

Thus, $\quad b_{i}^{0}-b_{i}^{1}=\left(\frac{b_{j}^{\prime}}{b_{i}^{\prime}}\right)^{\frac{1}{d-1}}\left(b_{j}^{0}-b_{j}^{1}\right), \quad$ and thus, $\quad \frac{b_{i}^{0}-b_{i}^{1}}{b_{j}^{0}-b_{j}^{1}}=\left(\frac{b_{j}^{\prime}}{b_{i}^{\prime}}\right)^{\frac{1}{d-1}}$.

That is: $\left(b_{i}^{\prime} / b_{j}^{\prime}\right)=\left(b_{j}^{\prime} / b_{i}^{\prime}\right)^{\frac{1}{d-1}}$. Thus, $\left(b_{i}^{\prime} / b_{j}^{\prime}\right)^{d-1}=\left(b_{j}^{\prime} / b_{i}^{\prime}\right)=\left(b_{i}^{\prime} / b_{j}^{\prime}\right)^{-1}$. At this point, there are two possibilities: either $b_{i}^{\prime} / b_{j}^{\prime}= \pm 1$, or $d=0$. But $d>0$ by hypothesis, so we must have $b_{i}^{\prime} / b_{j}^{\prime}= \pm 1$. Substituting this into (E24), we get $b_{i}^{s} / b_{j}^{s}=( \pm 1)^{\frac{1}{d-1}}$. The case $b_{i}^{\prime} / b_{j}^{\prime}=-1$ is possible only if $\frac{1}{d-1}$ is an integer. In any event, we have $b_{i}^{s} / b_{j}^{s}= \pm 1$ for all $s \in[0,1]$. In particular, setting $s=0$ we have $b_{i}^{0}= \pm b_{j}^{0}$. But this contradicts our initial hypotheses that $b_{i}^{0} \neq \pm b_{j}^{0}$.

To avoid this contradiction, we must have $d=1$.

Proof of Theorem 4.1. (a) Proposition 3.6 implies that the median rule is upper hemicontinuous, because the function $\phi^{1}(r)=r$ is continuous on $[-1,1]$. Clearly, $\phi^{1}$ is regular. It remains to show that the median rule satisfies reinforcement. 
To see this, let $\mu_{1}, \mu_{2} \in \Delta(\mathcal{X})$, and let $\mu=c_{1} \mu_{1}+c_{2} \mu_{2}$ for some $c_{1}, c_{2} \in[0,1]$ with $c_{1}+c_{2}=$ 1. Let $\mathbf{x} \in \operatorname{Median}\left(\mathcal{X}, \mu_{1}\right) \cap \operatorname{Median}\left(\mathcal{X}, \mu_{2}\right)$; we must show that $\mathbf{x} \in \operatorname{Median}(\mathcal{X}, \mu)$.

Observe that $\widetilde{\mu}=c_{1} \widetilde{\mu}_{1}+c_{2} \widetilde{\mu}_{2}$. Thus, for any $\mathbf{y} \in \mathcal{X}$, we have

$$
\mathbf{y} \bullet \widetilde{\mu}=c_{1} \mathbf{y} \bullet \widetilde{\mu}_{1}+c_{2} \mathbf{y} \bullet \widetilde{\mu}_{2} \underset{\left(_{*}\right)}{\leq} c_{1} \mathbf{x} \bullet \widetilde{\mu}_{1}+c_{2} \mathbf{x} \bullet \widetilde{\mu}_{2}=\mathbf{x} \bullet \widetilde{\mu} .
$$

(Here, $(*)$ is because $\mathbf{y} \bullet \widetilde{\mu}_{1} \leq \mathbf{x} \bullet \widetilde{\mu}_{1}$ and $\mathbf{y} \bullet \widetilde{\mu}_{2} \leq \mathbf{x} \bullet \widetilde{\mu}_{2}$, because $\mathbf{x} \in \operatorname{Median}\left(\mathcal{X}, \mu_{1}\right)$ and $\mathbf{x} \in \operatorname{Median}\left(\mathcal{X}, \mu_{2}\right)$.) Thus, we conclude that $\mathbf{y} \bullet \widetilde{\mu} \leq \mathbf{x} \bullet \widetilde{\mu}$ for all $\mathbf{y} \in \mathcal{X}$. Thus, $\mathbf{x} \in \operatorname{Median}(\mathcal{X}, \mu)$, as desired.

(b) "£" is a special case of part (a).

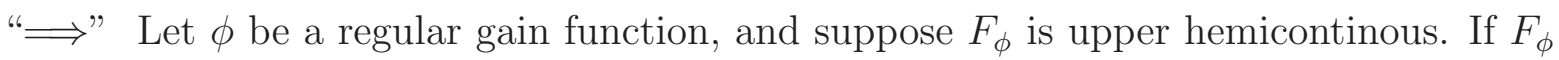
satisfies reinforcement, then $F_{\phi}$ satisfies neutral reinforcement. (Observe that $F_{\phi}\left(\delta_{\mathbf{x}, \mathbf{y}}\right) \supseteq$ $\{\mathbf{x}, \mathbf{y}\}$. Thus, if $F_{\phi}(\mu)=\{\mathbf{x}, \mathbf{y}\}$, then reinforcement implies that $F_{\phi}\left(c_{1} \mu+c_{2} \delta_{\mathbf{x}, \mathbf{y}}\right)=$ $\{\mathbf{x}, \mathbf{y}\}$ for any $c_{1}, c_{2}>0$ with $c_{1}+c_{2}=1$.) Thus, Theorem 5.1 implies that $F_{\phi}=H^{d}$ for some $d \in(0, \infty)$. Now Lemma E.11 implies that $F_{\phi}$ is the median rule.

Proof of Proposition 4.2. Let $\mathcal{X}^{\prime}:=\max (\mathcal{X}, \underset{\mu}{\prec})$. Clearly, Median $(\mathcal{X}, \mu) \subseteq \mathcal{X}^{\prime}$. We must show that Median $(\mathcal{X}, \mu) \supseteq \mathcal{X}^{\prime}$. Suppose $\mathbf{x} \notin \operatorname{Median}(\mathcal{X}, \mu)$; we will show that $\mathbf{x} \notin \mathcal{X}^{\prime}$. To do this, let $\mathcal{Y}(\mathbf{x}):=\{\mathbf{y} \in \mathcal{X} ; \mathbf{y} \underset{\mathcal{X}}{\bar{x}} \mathbf{x}$; we must find some $\mathbf{y} \in \mathcal{Y}(\mathbf{x})$ such that $\mathbf{x} \underset{\mu}{\sim} \mathbf{y}$.

Let $\mathcal{C}:=\operatorname{conv}(\mathcal{X})$. For any $\mathbf{x}, \mathbf{y} \in \mathcal{X}$, define $\mathcal{C}_{\mathbf{x}}:=\{\mathbf{c} \in \mathcal{C} ; \mathbf{x} \in \operatorname{Median}(\mathbf{c})\}$ and $\mathcal{B}_{\mathbf{x}, \mathbf{y}}:=$ $\mathcal{C}_{\mathbf{x}} \cap \mathcal{C}_{\mathbf{y}}=\{\mathbf{c} \in \mathcal{C} ;\{\mathbf{x}, \mathbf{y}\} \in \operatorname{Median}(\mathbf{c})\}$. Then $\mathcal{C}_{\mathbf{x}}$ and $\mathcal{B}_{\mathbf{x}, \mathbf{y}}$ are closed, because the median rule is upper hemicontinuous by Corollary 3.6. The 'internal boundary' of $\mathcal{C}_{\mathbf{x}}$ is the set $\partial_{*} \mathcal{C}_{\mathbf{x}}:=\mathcal{C}_{\mathbf{x}} \cap \operatorname{cl}\left(\mathcal{C} \backslash \mathcal{C}_{\mathbf{x}}\right)$.

Claim 1: $\quad \partial_{*} \mathcal{C}_{\mathbf{x}}=\bigcup_{\mathbf{y} \in \mathcal{Y}(\mathbf{x})} \mathcal{B}_{\mathbf{x}, \mathbf{y}}$

Proof: " $\supseteq$ " is clear by definition.

"ᄃ" Let $D:=\operatorname{dim}(\mathcal{C})$; then $\operatorname{dim}\left(\partial_{*} \mathcal{C}_{\mathbf{x}}\right)=D-1$, because it is the boundary of a relatively open subset of $\mathcal{C}$. We have

$$
\begin{aligned}
\partial_{*} \mathcal{C}_{\mathbf{x}} & =\mathcal{C}_{\mathbf{x}} \cap\left(\bigcup_{\mathbf{y} \in \mathcal{X} \backslash\{\mathbf{x}\}} \mathcal{C}_{\mathbf{y}}\right)=\bigcup_{\mathbf{y} \in \mathcal{X} \backslash\{\mathbf{x}\}}\left(\mathcal{C}_{\mathbf{x}} \cap \mathcal{C}_{\mathbf{y}}\right) \\
& =\bigcup_{\mathbf{y} \in \mathcal{X} \backslash\{\mathbf{x}\}} \mathcal{B}_{\mathbf{x}, \mathbf{y}}=\overbrace{\bigcup_{\mathbf{y} \in \mathcal{Y}(\mathbf{x})}^{(\mathrm{A})} \mathcal{B}_{\mathbf{x}, \mathbf{y}}} \cup \overbrace{\bigcup_{\mathbf{y} \in \mathcal{X} \backslash \mathcal{Y}(\mathbf{x})}^{(\mathrm{B})} \mathcal{B}_{\mathbf{x}, \mathbf{y}}}
\end{aligned}
$$

Now, $\mathcal{X} \backslash \mathcal{Y}(\mathbf{x})$ is a finite set, and every element in union (B) is a set of dimension $D-2$ or less (because it is defined by two or more linear constraints); thus union (B) is nowhere dense in $\partial_{*} \mathcal{C}_{\mathbf{x}}$. Thus, union (A) must be dense in $\partial_{*} \mathcal{C}_{\mathbf{x}}$. But union (A) is also closed (it is a finite union of closed sets). Thus, union (A) must cover all of $\partial_{*} \mathcal{C}_{\mathbf{x}}$. $\diamond$ Claim 1 
Let $\mathcal{D}:=\left\{\mathbf{r} \in \mathbb{R}^{\mathcal{K}} ; \quad(\mathbf{y}-\mathbf{x}) \bullet \mathbf{r} \leq 0\right.$, for all $\left.\mathbf{y} \in \mathcal{Y}(\mathbf{x})\right\} ;$ then $\mathcal{D}$ is a closed, convex polyhedron.

Claim 2: $\quad \mathcal{C}_{\mathrm{x}}=\mathcal{D} \cap \mathcal{C}$.

Proof: $\mathcal{C}_{\mathbf{x}}:=\{\mathbf{c} \in \mathcal{C} ; \quad(\mathbf{y}-\mathbf{x}) \bullet \mathbf{r} \leq 0$, for all $\mathbf{y} \in \mathcal{X}\} ;$ thus $\mathcal{C}_{\mathbf{x}} \subseteq \mathcal{D} \cap \mathcal{C}$. We must show that $\mathcal{C}_{\mathbf{x}} \supseteq \mathcal{D} \cap \mathcal{C}$. Suppose $\mathbf{c} \notin \mathcal{C}_{\mathbf{x}}$; we will show that $\mathbf{c} \notin \mathcal{D} \cap \mathcal{C}$. Clearly $\mathbf{x} \in \mathcal{C}_{\mathbf{x}}$. Let $\mathcal{L}$ be the line segment connecting $\mathbf{c}$ to $\mathbf{x}$. One end of $\mathcal{L}$ is inside $\mathcal{C}_{\mathbf{x}}$, while the other end is in $\mathcal{C}_{\mathbf{y}}$ for some $\mathbf{y} \in \mathcal{X} \backslash\{\mathbf{x}\}$. Thus, $\mathcal{L}$ intersects $\partial_{*} \mathcal{C}_{\mathbf{x}}$ at some point $\mathbf{b}$ (because the median rule is upper hemicontinuous). Thus, Claim 1 says that $\mathbf{b} \in \mathcal{B}_{\mathbf{x}, \mathbf{y}}$ for some $\mathbf{y} \in \mathcal{Y}(\mathbf{x})$. Thus, $(\mathbf{y}-\mathbf{x}) \bullet \mathbf{b}=0$. Since $(\mathbf{y}-\mathbf{x}) \bullet \mathbf{x}<0$, we must have $(\mathbf{y}-\mathbf{x}) \bullet \mathbf{c}>0$, which means that $\mathbf{c} \notin \mathcal{D}$.

$\diamond$ Claim 2

Now, $\mathbf{x} \notin \operatorname{Median}(\mathcal{X}, \mu)$, so $\widetilde{\mu} \notin \mathcal{C}_{\mathbf{x}}$; hence Claim 2 implies that $\widetilde{\mu} \notin \mathcal{D}$ (because $\widetilde{\mu} \in \mathcal{C}$ ). Since $\mathcal{D}$ is closed and convex, the Separating Hyperplane Theorem yields some $\mathbf{v} \in \mathbb{R}^{\mathcal{K}}$ such that $\mathbf{v} \bullet \widetilde{\mu}>0 \geq \mathbf{v} \bullet \mathbf{d}$ for all $\mathbf{d} \in \mathcal{D}$. A standard variant of Farkas' Lemma says that $\mathbf{v}$ can be written as a nonnegative linear combination of the constraint vectors defining $\mathcal{D}$ - that is, there exist nonnegative coefficients $p_{\mathbf{y}} \geq 0$ for all $\mathbf{y} \in \mathcal{Y}(\mathbf{x})$, such that $\mathbf{v}=\sum_{\mathbf{y} \in \mathcal{Y}(\mathbf{x})} p_{\mathbf{y}}(\mathbf{y}-\mathbf{x})$ (Bertsimas and Tsitsiklis, 1997, Theorem 4.7, p.166). But then we have

$$
0<\mathbf{v} \bullet \widetilde{\mu}=\sum_{\mathbf{y} \in \mathcal{Y}(\mathbf{x})} p_{\mathbf{y}}(\mathbf{y}-\mathbf{x}) \bullet \widetilde{\mu} .
$$

Now, $p_{\mathbf{y}} \geq 0$ for all $\mathbf{y} \in \mathcal{Y}(\mathbf{x})$, so we must have $(\mathbf{y}-\mathbf{x}) \bullet \widetilde{\mu}>0$ for some $\mathbf{y} \in \mathcal{Y}(\mathbf{x})$. But this means that $\mathbf{x} \underset{\mu}{\stackrel{y}{ }} \mathbf{y}$. Thus, $\mathbf{x}$ is not maximal in $(\mathcal{X}, \underset{\mu}{\stackrel{\prec}{\mu}})$; hence $\mathbf{x} \notin \mathcal{X}^{\prime}$.

\section{Proofs from $\S 2$.}

Proof of Theorem 2.1(a). In $\S 4$, we defined the 'internal graph' as a binary relation $\overline{\bar{x}}$ on $\mathcal{X}$; we also defined an orientation $\underset{\mu}{\sim}$ on this graph by setting $\mathbf{x} \underset{\mu}{\vec{y}} \mathbf{y}$ if $\mathbf{x} \bullet \widetilde{\mu}<\mathbf{y} \bullet \widetilde{\mu}$

Claim 1: For any $\mu \in \Delta(\mathcal{X})$, any odd increasing $\phi:[-1,1] \longrightarrow \mathbb{R}$, and any $\mathbf{x} \overline{\overline{\mathcal{X}}} \mathbf{y}$, we have $(\mathrm{x} \bullet \phi(\widetilde{\mu})<\mathbf{y} \bullet \phi(\widetilde{\mu})) \Longleftrightarrow\left(\mathbf{x}_{\mu}^{\leftrightarrow} \mathbf{y}\right)$.

Proof: Since $\mathcal{X}$ is proximal, there are only two cases.

If $d(\mathbf{x}, \mathbf{y})=1$ and $\mathcal{K}_{ \pm}(\mathbf{x}, \mathbf{y})=\{k\}$, then $(\mathbf{x}-\mathbf{y}) \bullet \phi(\widetilde{\mu})=\left(x_{k}-y_{k}\right) \phi\left(\widetilde{\mu}_{k}\right)$. Thus,

$$
\begin{aligned}
(\mathbf{x} \bullet \phi(\widetilde{\mu})<\mathbf{y} \bullet \phi(\widetilde{\mu})) & \Longleftrightarrow\left(\left(x_{k}-y_{k}\right) \cdot \phi\left(\widetilde{\mu}_{k}\right)<0\right) \\
& \Longleftrightarrow\left(\left(x_{k}-y_{k}\right) \cdot \widetilde{\mu}_{k}<0\right) \Longleftrightarrow(\mathbf{x} \underset{\mu}{\rightleftarrows} \mathbf{y}) .
\end{aligned}
$$

$(*)$ is because $\phi$ is sign-preserving because $\phi$ is increasing and $\phi(0)=0$ (because $\phi$ is odd). 
If $d(\mathbf{x}, \mathbf{y})=2$, and $\mathcal{K}_{ \pm}(\mathbf{x}, \mathbf{y})=\{i, j\}$, then $(\mathbf{x}-\mathbf{y}) \bullet \phi(\widetilde{\mu})=\left(x_{i}-y_{i}\right) \phi\left(\widetilde{\mu}_{i}\right)+\left(x_{j}-y_{j}\right) \phi\left(\widetilde{\mu}_{j}\right)$. Thus,

$$
\begin{aligned}
(\mathbf{x} \bullet \phi(\widetilde{\mu})<\mathbf{y} \bullet \phi(\widetilde{\mu})) & \Longleftrightarrow\left(\left(x_{i}-y_{i}\right) \cdot \phi\left(\widetilde{\mu}_{i}\right)<\left(y_{j}-x_{j}\right) \cdot \phi\left(\widetilde{\mu}_{j}\right)\right) \\
& \Longleftrightarrow\left(\left(x_{i}-y_{i}\right) \cdot \widetilde{\mu}_{i}<\left(y_{j}-x_{j}\right) \cdot \widetilde{\mu}_{j}\right) \Longleftrightarrow(\mathbf{x} \underset{\mu}{y}) .
\end{aligned}
$$

Here, $(*)$ is because $\phi$ is odd and increasing, and $\left|x_{i}-y_{i}\right|=\left|x_{j}-y_{j}\right|=2 . \quad \diamond$ claim 1

Let $\underset{\mu}{\prec}$ be the transitive closure of $\underset{\mu}{\sim}$, and let $\mathcal{X}^{\prime}:=\max (\mathcal{X}, \underset{\mu}{\prec})$. Proposition 4.2 says $\operatorname{Median}(\mathcal{X}, \mu)=\mathcal{X}^{\prime}$.

Claim 2: If $\phi:[-1,1] \longrightarrow \mathbb{R}$ is odd and increasing, then $F_{\phi}(\mathcal{X}, \mu) \subseteq \mathcal{X}^{\prime}$.

Proof: For any $\mathbf{x}, \mathbf{y} \in \mathcal{X}$ (adjacent or not), write $\mathbf{x} \frac{\wp}{\phi} \mathbf{y}$ if $\mathbf{x} \bullet \phi(\widetilde{\mu}) \leq \mathbf{y} \bullet \phi(\widetilde{\mu})$. This is a total ordering of $\mathcal{X}$, and $F_{\phi}(\mathcal{X}, \mu)=\max (\mathcal{X}, \underset{\phi}{\varsigma})$. However, Claim 1 shows that $\vec{\mu}$ is a sub-relation of $\underset{\phi}{\prec}$ (the asymmetric part of $\underset{\phi}{\prec}$ ); thus, $\underset{\mu}{\prec}$ is also a sub-relation of $\underset{\phi}{\prec}, \operatorname{so~} \max (\mathcal{X}, \underset{\phi}{\prec}) \subseteq \max (\mathcal{X}, \underset{\mu}{\prec})$.

$\diamond$ Claim 2

Claim 3: If $\phi:[-1,1] \longrightarrow \mathbb{R}$ is odd, increasing and continuous, then $F_{\phi}(\mathcal{X}, \mu)=$ $\operatorname{Median}(\mathcal{X}, \mu)$ for all $\mu \in \Delta(\mathcal{X})$.

Proof: $F_{\phi}$ is upper hemicontinuous on $\Delta(\mathcal{X})$ by Proposition 3.6, because $\phi$ is continuous on $[-1,1]$. The median rule is monotone by Proposition 3.5(b), because the function $\eta(x)=x$ is strictly increasing. Claim 2 and Proposition 4.2 imply that $F_{\phi}(\mathcal{X}, \mu) \subseteq$ Median $(\mathcal{X}, \mu)$ for all $\mu \in \Delta(\mathcal{X})$. Thus, Lemma C.1 shows that in fact $F_{\phi}(\mathcal{X}, \mu)=$ $\operatorname{Median}(\mathcal{X}, \mu)$ for all $\mu \in \Delta(\mathcal{X})$.

$\diamond$ Claim 3

Claim 4: (a) If $\psi:[-1,1] \longrightarrow \mathbb{R}$ is any odd, increasing, continuous function, then $\operatorname{SSME}(\mathcal{X}, \mu)=F_{\psi}(\mathcal{X}, \mu)$ for all $\mu \in \Delta(\mathcal{X})$.

(b) In particular, $\operatorname{SSME}(\mathcal{X}, \mu)=\operatorname{Median}(\mathcal{X}, \mu)$ for all $\mu \in \Delta(\mathcal{X})$.

Proof: Let $\Phi_{I}$ be the set of all odd, continuous, increasing functions from $[-1,1]$ into $\mathbb{R}$. Then

$$
\begin{aligned}
\operatorname{SSME}(\mathcal{X}, \mu) & \overline{\overline{(*)}} \bigcup_{\phi \in \Phi_{I}} F_{\phi}(\mathcal{X}, \mu) \overline{\overline{(\dagger)}} \bigcup_{\phi \in \Phi_{I}} \operatorname{Median}(\mathcal{X}, \mu) \\
& =\operatorname{Median}(\mathcal{X}, \mu) \overline{\overline{(\dagger)}} F_{\psi}(\mathcal{X}, \mu) .
\end{aligned}
$$

Here, $(*)$ is by Proposition 1.1(b), and both $(\dagger)$ are by Claim 3.

$\diamond$ Claim 4

Claim 5: $\quad \gamma_{\mu, \mathbf{x}}=\gamma_{\mu, \mathbf{y}}$ for all $\mathbf{x}, \mathbf{y} \in \operatorname{SSME}(\mathcal{X}, \mu)$.

Proof: By contradiction, suppose there exist $\mathbf{x}, \mathbf{y} \in \operatorname{SSME}(\mathcal{X}, \mu)$ with $\gamma_{\mu, \mathbf{x}} \neq \gamma_{\mu, \mathbf{y}}$. Then we can find some continuous, increasing $\phi:[-1,1] \longrightarrow \mathbb{R}$ such that $\phi^{*}\left(\gamma_{\mu, \mathbf{x}}\right) \neq \phi^{*}\left(\gamma_{\mu, \mathbf{y}}\right)$. Thus, Lemma D.2(c) implies that at most one of $\mathbf{x}$ or $\mathbf{y}$ is in $F_{\psi}(\mathcal{X}, \mu)$, contradicting Claim 4(a). 
Claim 6: $\operatorname{SME}(\mathcal{X}, \mu) \subseteq \operatorname{SSME}(\mathcal{X}, \mu)$ for all $\mu \in \Delta(\mathcal{X})$.

Proof: By contrapositive, suppose $\mathbf{z} \in \mathcal{X} \backslash \operatorname{SSME}(\mathcal{X}, \mu)$. Then there exists some $\rho \in$ $\Delta(\mathcal{X})$ such that $\mathbf{z} \underset{\mu}{\triangleleft} \rho$-i.e. such that $\gamma_{\mu, \mathbf{z}} \leq \gamma_{\mu, \rho}$ with at least one strict inequality. Without loss of generality, suppose that $\rho$ is $(\underset{\mu}{\triangleleft})$-maximal in $\Delta(\mathcal{X})$. Then $\operatorname{supp}(\rho) \subseteq$ $\operatorname{SSME}(\mathcal{X}, \mu)$. Fix $\mathbf{y} \in \operatorname{SSME}(\mathcal{X}, \mu)$; then Claim 5 says that $\gamma_{\mu, \mathbf{x}}=\gamma_{\mu, \mathbf{y}}$ for all other $\mathbf{x} \in \operatorname{SSME}(\mathcal{X}, \mu)$; thus, $\gamma_{\mu, \rho}=\gamma_{\mu, \mathbf{y}}$ (because $\gamma_{\mu, \rho}$ is a convex combination of $\left\{\gamma_{\mu, \mathbf{x}}\right.$; $\mathbf{x} \in \operatorname{SSME}(\mathcal{X}, \mu)\})$. Thus, $\gamma_{\mu, \mathbf{z}} \leq \gamma_{\mu, \mathbf{y}}$, with at least one strict inequality. Thus, $\mathbf{z}{ }_{\mu}^{\triangleleft} \mathbf{y}$. Thus, $\mathbf{z} \notin \operatorname{SME}(\mathcal{X}, \mu)$.

$\diamond$ Claim 6

Now Proposition 1.1(a) and Claim 6 imply that $\operatorname{SSME}(\mathcal{X}, \mu)=\operatorname{SME}(\mathcal{X}, \mu)$. Then Claim 5 implies that $\mathcal{X}$ is supermajoritarian determinate.

(b) (by contrapositive) Define $\phi(r):=r$ for all $r \in[-1,1]$ (so $F_{\phi}$ is the median rule). Suppose $\mathcal{X}$ is not proximal. Then there exist $\mathbf{x}, \mathbf{y} \in \mathcal{X}$ with $d(\mathbf{x}, \mathbf{y}) \geq 3$, such that ${ }^{o} \mathcal{B}_{\mathbf{x}, \mathbf{y}}^{\phi} \neq \emptyset$. Thus, if $k \in \mathcal{K}_{ \pm}(\mathbf{x}, \mathbf{y})$, then $\mathcal{R}_{\mathbf{x}, \mathbf{y}}^{k} \neq \emptyset$; thus, $\mathcal{R}_{\mathcal{X}}^{\phi} \neq \emptyset$.

Now, the median rule satisfies neutral reinforcement, by Theorem 5.1(a). Thus, $\mathcal{R}_{\mathcal{X}}^{\phi} \cup\{0\}$ is connected, by Lemma ??. Thus, Theorem 6.4 is applicable. Let $\psi:[-1,1] \longrightarrow \mathbb{R}$ be another odd, continuous, increasing function, such that $\psi$ is not a scalar multiple of $\phi$ on $\mathcal{R}_{\mathcal{X}}^{\phi}$. Then the contrapositive of Theorem 6.4 implies that $F_{\phi} \neq F_{\psi}$. Thus, $\mathcal{X}$ is not supermajoritarian determinant.

Proof of Proposition 2.3. Let $\mathbf{x}, \mathbf{z} \in \mathcal{X}^{\text {com }}$ with $d(\mathbf{x}, \mathbf{z}) \geq 3$; we must show that $\mathbf{x}$ 寿 $\mathbf{z}$. In fact, we will show that $\mathbf{x} \underset{\mathcal{X}}{ } \mathbf{y}$ (see Appendix B). We define

$$
\begin{aligned}
& \mathcal{K}_{++}:=\left\{k \in \mathcal{K} ; x_{k}=1=z_{k}\right\}, \quad \mathcal{K}_{+-}:=\left\{k \in \mathcal{K} ; x_{k}=1, z_{k}=-1\right\} \\
& \mathcal{K}_{--}:=\left\{k \in \mathcal{K} ; x_{k}=-1=z_{k}\right\}, \quad \text { and } \quad \mathcal{K}_{-+}:=\left\{k \in \mathcal{K} ; x_{k}=-1, z_{k}=1\right\} .
\end{aligned}
$$

We have $|\mathbf{x}|=\left|\mathcal{K}_{++}\right|+\left|\mathcal{K}_{+-}\right|$and $|\mathbf{z}|=\left|\mathcal{K}_{++}\right|+\left|\mathcal{K}_{-+}\right|$. Thus,

$$
I-\left|\mathcal{K}_{++}\right| \leq\left|\mathcal{K}_{+-}\right|,\left|\mathcal{K}_{-+}\right| \leq J-\left|\mathcal{K}_{++}\right|
$$

Now, $\left|\mathcal{K}_{+-}\right|+\left|\mathcal{K}_{-+}\right|=d(\mathbf{x}, \mathbf{z}) \geq 3$, so either $\left|\mathcal{K}_{+-}\right| \geq 2$ or $\left|\mathcal{K}_{-+}\right| \geq 2$. If $\left|\mathcal{K}_{+-}\right| \geq 2$, then let $\emptyset \neq \mathcal{K}_{+-}^{1} \subsetneq \mathcal{K}_{+-}$; otherwise set $\mathcal{K}_{+-}^{1}:=\emptyset$. If $\left|\mathcal{K}_{-+}\right| \geq 2$, then let $\emptyset \neq \mathcal{K}_{-+}^{1} \subsetneq \mathcal{K}_{-+}$; otherwise set $\mathcal{K}_{-+}^{1}:=\emptyset$. Let $\mathcal{K}_{-+}^{2}:=\mathcal{K}_{-+} \backslash \mathcal{K}_{-+}^{1}$ and $\mathcal{K}_{+-}^{2}:=\mathcal{K}_{+-} \backslash \mathcal{K}_{+-}^{1}$. Thus, $\left|\mathcal{K}_{+-}\right|=\left|\mathcal{K}_{+-}^{1}\right|+\left|\mathcal{K}_{+-}^{2}\right|$ and $\left|\mathcal{K}_{-+}\right|=\left|\mathcal{K}_{-+}^{1}\right|+\left|\mathcal{K}_{-+}^{2}\right|$. We can always choose these sets so that

$$
\text { and } \quad \begin{aligned}
I-\left|\mathcal{K}_{++}\right| \leq\left|\mathcal{K}_{+-}^{1}\right|+\left|\mathcal{K}_{-+}^{2}\right| \leq J-\left|\mathcal{K}_{++}\right| \\
I-\left|\mathcal{K}_{++}\right| \leq\left|\mathcal{K}_{+-}^{2}\right|+\left|\mathcal{K}_{-+}^{1}\right| \leq J-\left|\mathcal{K}_{++}\right|
\end{aligned}
$$

Now define $\mathbf{y}^{1}, \mathbf{y}^{2} \in \mathbb{N}^{L}$ as follows:

$$
\begin{aligned}
y_{k}^{1} & :=\left\{\begin{array}{rll}
1 & \text { if } & k \in \mathcal{K}_{+-}^{1} \sqcup \mathcal{K}_{++} \sqcup \mathcal{K}_{-+}^{2} ; \\
-1 & \text { if } & k \in \mathcal{K}_{+-}^{2} \sqcup \mathcal{K}_{--} \sqcup \mathcal{K}_{-+}^{1} ;
\end{array}\right. \\
\text { and } \quad y_{k}^{2} & :=\left\{\begin{array}{rll}
1 & \text { if } & k \in \mathcal{K}_{+-}^{2} \sqcup \mathcal{K}_{++} \sqcup \mathcal{K}_{-+}^{1} \\
-1 & \text { if } & k \in \mathcal{K}_{+-}^{1} \sqcup \mathcal{K}_{--} \sqcup \mathcal{K}_{-+}^{2}
\end{array}\right.
\end{aligned}
$$


Then $I \leq\left|\mathbf{y}^{1}\right|,\left|\mathbf{y}^{2}\right| \leq J$, so $\mathbf{y}^{1}, \mathbf{y}^{2} \in \mathcal{X}^{\text {com }}$. For every $k \in \mathcal{K}$, we have

$$
y_{k}^{1}+y_{k}^{2}=\left\{\begin{array}{rll}
2 & \text { if } & k \in \mathcal{K}_{++} \\
0 & \text { if } & k \in \mathcal{K}_{+-} \sqcup \mathcal{K}_{-+} \\
-2 & \text { if } & k \in \mathcal{K}_{--}
\end{array}\right\}=x_{k}+z_{k} .
$$

Thus, $\left(\mathbf{y}^{1}+\mathbf{y}^{2}\right) / 2=(\mathbf{x}+\mathbf{z}) / 2$, so $\operatorname{conv}\{\mathbf{x}, \mathbf{z}\} \cap \operatorname{conv}\left\{\mathbf{y}^{1}, \mathbf{y}^{2}\right\} \neq \emptyset$, so the line segment $\operatorname{conv}\{\mathbf{x}, \mathbf{z}\}$ is not an edge of $\operatorname{conv}(\mathcal{X})$. Thus, $\mathbf{x} \overleftarrow{\mathcal{X}}_{\mathcal{X}} \mathbf{y}$. Thus, Proposition B.1(c) says that $\mathbf{x}$ 表 $\mathbf{z}$.

This holds whenever $d(\mathbf{x}, \mathbf{y}) \geq 3$. Thus, $\mathcal{X}^{\text {com }}$ is proximal.

\section{References}

Anderson, R. M., 1991. Nonstandard analysis with applications to economics. In: Handbook of mathematical economics, Vol. IV. North-Holland, Amsterdam, pp. 2145-2208.

Barthélémy, J.-P., 1989. Social welfare and aggregation procedures: combinatorial and algorithmic aspects. In: Applications of combinatorics and graph theory to the biological and social sciences. Vol. 17 of IMA Vol. Math. Appl. Springer, New York, pp. 39-73.

Barthélémy, J.-P., Janowitz, M. F., 1991. A formal theory of consensus. SIAM J. Discrete Math. 4 (3), $305-322$.

Barthélémy, J.-P., Monjardet, B., 1981. The median procedure in cluster analysis and social choice theory. Math. Social Sci. 1 (3), 235-267.

Barthélémy, J.-P., Monjardet, B., 1988. The median procedure in data analysis: new results and open problems. In: Classification and related methods of data analysis (Aachen, 1987). North-Holland, Amsterdam, pp. 309-316.

Bertsimas, D., Tsitsiklis, J. N., 1997. Introduction to Convex Optimization. Athena Scientific, Belmont, MA.

Condorcet, M. d., 1785. Essai sur l'application de l'analyse à la probabilité des décisions rendues à la pluralité des voix. Paris.

Guilbaud, G.-T., Octobre-Déecmbre 1952. Les théories de l'intérêt général et le problème logique de l'aggrégation. Economie Appliquée V (4), 501-551.

Kemeny, J. G., Fall 1959. Math without numbers. Daedalus 88, 571-591.

Kolmogorov, A. N., Fomīn, S. V., 1975. Introductory real analysis. Dover Publications Inc., New York, translated from the second Russian edition and edited by Richard A. Silverman, Corrected reprinting.

List, C., Pettit, P., 2002. Aggregating sets of judgements: an impossibility result. Economics and Philosophy $18,89-110$.

List, C., Puppe, C., 2009. Judgement aggregation: a survey. In: Oxford handbook of rational and social choice. Oxford University Press, Oxford, UK., pp. 457-482.

Nehring, K., Pivato, M., 2011a. Incoherent majorities: the McGarvey problem in judgement aggregation. Discrete Applied Mathematics 159, 1488-1507. 
Nehring, K., Pivato, M., 2011b. Supermajoritarian efficient judgement aggregation. (preprint).

Nehring, K., Pivato, M., Puppe, C., July 2011. Condorcet admissibility: Indeterminacy and pathdependence under majority voting on interconnected decisions. (preprint).

Nehring, K., Puppe, C., 2007. The structure of strategy-proof social choice I: General characterization and possibility results on median spaces. J.Econ.Theory 135, 269-305.

Rubinstein, A., Fishburn, P. C., 1986. Algebraic aggregation theory. J. Econom. Theory 38 (1), 63-77.

Tideman, T. N., 1987. Independence of clones as a criterion for voting rules. Soc. Choice Welf. 4 (3), $185-206$.

Wilson, R., 1975. On the theory of aggregation. J. Econom. Theory 10 (1), 89-99.

Young, H. P., 1986. Optimal ranking and choice from pairwise decisions. In: Grofman, B., Owen, G. (Eds.), Information pooling and group decision making. JAI press, Greenwich, CT, pp. 113-122.

Young, H. P., 1988. Condorcet's theory of voting. American Political Science Review 82 (4), 1231-1244.

Young, H. P., Winter 1995. Optimal voting rules. Journal of Economic Perspectives 9 (1), 51-64.

Young, H. P., 1997. Group choice and individual judgments. In: Mueller, D. C. (Ed.), Perspectives on public choice: A handbook. Cambridge UP, Cambridge, UK, Ch. 9, pp. 181-200.

Young, H. P., Levenglick, A., 1978. A consistent extension of Condorcet's election principle. SIAM J. Appl. Math. 35 (2), 285-300.

Zavist, T. M., Tideman, T. N., 1989. Complete independence of clones in the ranked pairs rule. Soc. Choice Welf. 6 (2), 167-173. 